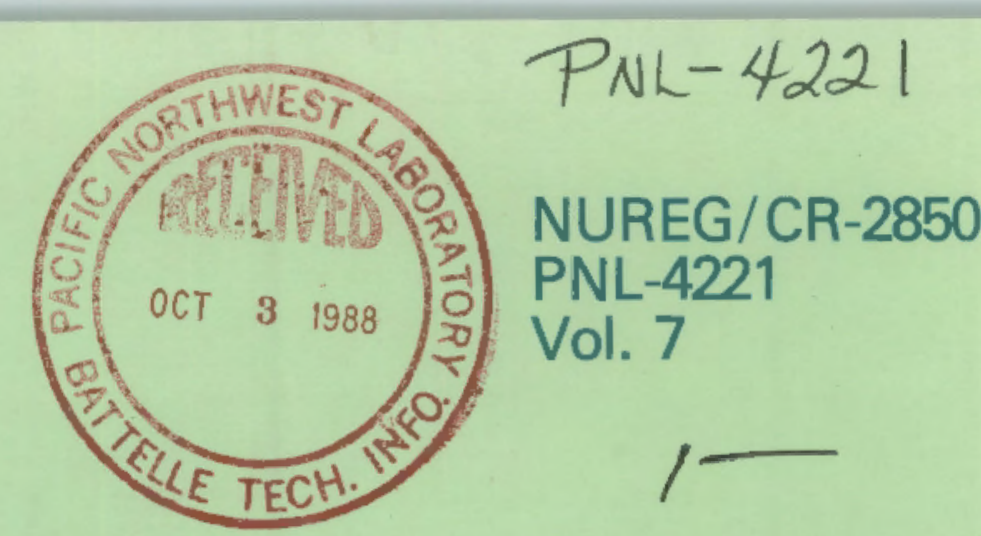

\title{
Population Dose Commitments Due to Radioactive Releases from Nuclear Power Plant Sites in 1985
}

Prepared by D. A. Baker

Pacific Northwest Laboratory

Operated by

Battelle Memorial Institute

Prepared for

U.S. Nuclear Regulatory

Commission 


\section{NOTICE}

This report was prepared as an account of work sponsored by an agency of the United States Government. Neither the United States Government nor any agency thereof, or any of their employees, makes any warranty, expressed or implied, or assumes any legal liability of re. sponsibility for any third party's use, or the results of such use, of any information, apparatus, product or process disclosed in this report, or represents that its use by such third party would not infringe privately owned rights.

\section{NOTICE}

Availability of Reference Materials Cited in NRC Publications

Most documents cited in NRC publications will be available from one of the following sources:

1. The NRC Public Document Room, 1717 H Street, N.W. Washington, DC 20555

2. The Superintendent of Documents, U.S. Government Printing Office, Post Office Box 37082 , Washington, DC 20013-7082

3. The National Technical Information Service, Springfield, VA 22161

Although the listing that follows represents the majority of documents cited in NRC publications, it is not intended to be exhaustive.

Referenced documents available for inspection and copying for a fee from the NRC Public Document Room include NRC correspcridence and internal NRC memoranda; NRC Office of Inspection and Enforcement bulletins, circulars, information notices, inspection and investigation notices: Licensee Event Reports; vendor reports and correspondence; Commission papers; and applicant and licensee documents and correspondence.

The following documents in the NUREG series are available for purchase from the GPO Sales Program: formal NRC staff and contractor reports, NRC-sponsored conference proceedings, and NRC booklets and brochures. Also available are Regulatory Guides, NRC regulations in the Code of Federal Regulations, and Nuclear Regulatory Commission /ssuances.

Documents available from the National Technical Information Service include NUREG series reports and technical reports prepared by other federal agencies and reports prepared by the Atomic Energy Commission, forerunner agency to the Nuclear Regulatory Commission.

Documents available from public and special technical libraries include all open literature items, such as books, journal and periodical articles, and transactions. Federal Register notices, federal and state legisiation, and congressional reports can usually be obtained from these libraries.

Documents such as theses, dissertations, foreign reports and translations, and non-NRC conference proceedings are available for purchase from the organization sponsoring the publication cited.

Single copies of NRC draft reports are available free, to the extent of supply, upon written request to the Division of Information Support Services, Distribution Section, U.S. Nuclear Regulatory Commission, Washington, DC 20555.

Copies of industry codes and standards used in a substantive manner in the NRC regulatory process are maintained at the NRC Library, 7920 Norfolk Avenue, Bethesda, Maryland, and are available there for reference use by the public. Codes and standards are usually copyrighted and may be purchased from the originating organization or, if they are American National Standards, from the American National Standards Institute, 1430 Broadway, New York, NY 10018. 
NUREG/CR-2850

PNL-4221

Vol. 7

\section{Population Dose Commitments Due to Radioactive Releases from Nuclear Power Plant Sites in 1985}

Manuscript Completed: August 1988

Date Published: August 1988

Prepared by

D. A. Baker

Pacific Northwest Laboratory

Richland, WA 99352

Prepared for

Office of Administration and Resources Management

U.S. Nuclear Regulatory Commission

Washington, DC 20555

NRC FIN B2243 


\section{PREVIOUS REPORTS IN THIS SERIES}

1. Population Dose Commitments Due to Radioactive Releases from Nuclear Power Plant Sites in 1975, PNL-2439, Octobier 1977.

2. Population Dose Commitments Due to Radioactive Releases from Nuclear Power Plant Sites in 1976, NUREG/CR-1125, PNL-2940, December 1979.

3. Population Dose Commitments Due to Radioactive Releases from Nuclear Power Plant Sites in 1977, NUREG/CR-1498, PNL-3324, October 1980.

4. Population Dose Commitments Due to Radioactive Releases from Nuclear Power Plant Sites in 1978, NUREG/CR-2201, PNL-4039, June 1982.

5. Population Dose Commitments Due to Radioactive Releases from Nuclear Power Plant Sites in 1979, NUREG/CR-2850, PNL-4221, Vol. 1, December 1982.

6. Population Dose Commitments Due to Radioactive Releases from Nuclear Power Plant Sites in 1980, NUREG/CR-2850, PNL-4221, Vo1. 2, August 1983.

7. Population Dose Commitments Due to Radioactive Releases from Nuclear Power Plant Sites in 1981, NUREG/CR-2850, PNL-4221, Vol. 3, January 1985.

8. Population Dose Commitments Due to Radioactive Releases from Nuclear Power Plant Sites in 1982, NUREG/CR-2850, PNL-4221, Vol. 4, June 1986.

9. Population Dose Commitments Due to Radioactive Releases from Nuclear Power Plant Sites in 1983, NUREG/CR-2850, PNL-4221, Vo1. 5, January 1987.

10. Population Dose Commitments Due to Radioactive Releases from Nuclear Power Plant Sites in 1984, NUREG/CR-2850, PNL-4221, Vol. 6, January 1988. 


\section{ABSTRACT}

Population radiation dose commitments have been estimated from reported radionuclide releases from commercial power reactors operating during 1985. Fifty-year dose commitments from a one-year exposure were calculated from both liquid and atmospheric releases for four population groups (infant, child, teen-ager and adult) residing between 2 and $80 \mathrm{~km}$ from each of 61 sites. This report tabulates the results of these calculations, showing the dose commitments for both liquid and airborne pathways for each age group and organ. Also included for each of the sites is a histogram showing the fraction of the total population within 2 to $80 \mathrm{~km}$ around each site receiving various average dose commitments from the airborne pathways.

The total dose commitments (from both liquid and airborne pathways) for each site ranged from a high of 73 person-rem to a low of 0.011 person-rem for the sites with plants operating throughout the year with an arithmetic mean of 3 person-rem. The total population dose for all sites was estimated at 200 person-rem for the $110 \mathrm{million}$ people considered at risk.

The site average individual dose commitment from all pathways ranged from a low of $5 \times 10^{-6}$ mrem to a high of $0.02 \mathrm{mrem}$. No attempt was made in this study to determine the maximum dose commitment received by any one individual from the radionuclides released at any of the sites. 



\section{CONTENTS}

ABSTRACT ............................ . . .

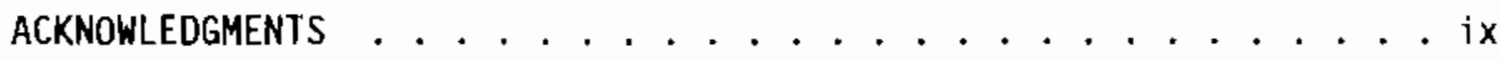

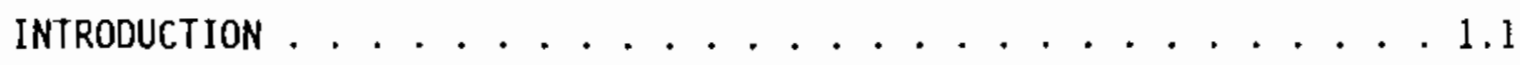

Site-Dependent Parameters . . . . . . . . . . . . . 1.4

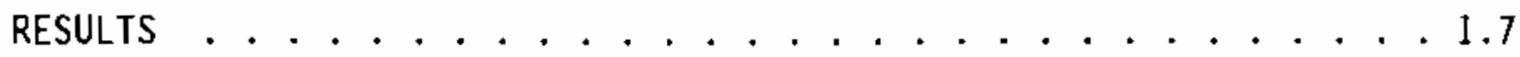

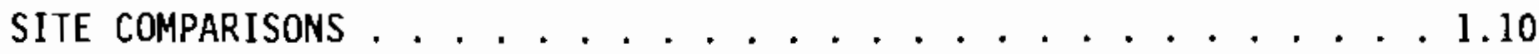

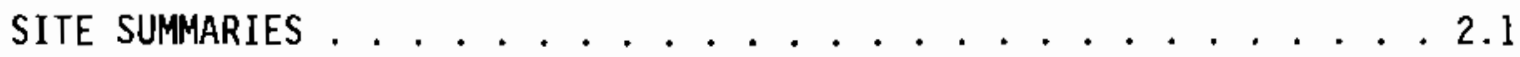

Arkansas 0 ne $1 \& 2 \ldots \ldots . \ldots . \ldots 2 . \ldots \ldots$

Beaver Valley . . . . . . . . . . . . . . . . . 2.4

Big Rock Point . . . . . . . . . . . . . 2.6

Browns Ferry 1, 2, \& $3 \ldots \ldots . . \ldots . . \ldots 2 . \ldots$

Brunswick $1 \& 2 \ldots \ldots . \ldots \ldots$

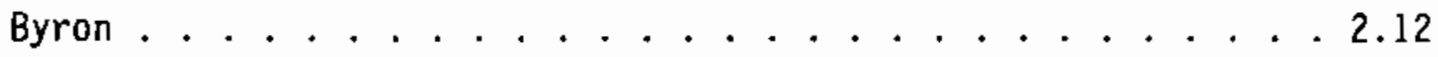

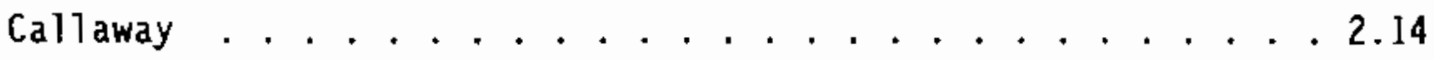

Calvert Cliffs $1 \& 2 \ldots \ldots \ldots . \ldots \ldots$

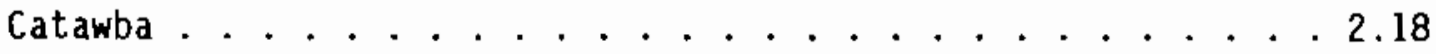

cook $1 \& 2 \ldots \ldots \ldots \ldots . \ldots \ldots$

Cooper ...................... . . 2.22

Crystal River 3..................... 2.24

Davis-Besse . . . . . . . . . . . . . . 2.26

Diablo Canyon $1 \& 2 \ldots \ldots . \ldots . . \ldots . . \ldots 28$

Dresden 1, 2, \& $3 \ldots \ldots \ldots . \ldots \ldots$ 


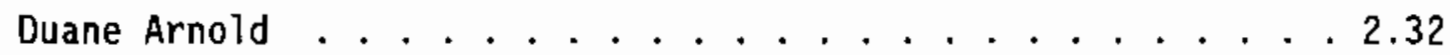

J. M. Farley $1 \& 2 \ldots \ldots . \ldots . \ldots . \ldots . \ldots . \ldots$

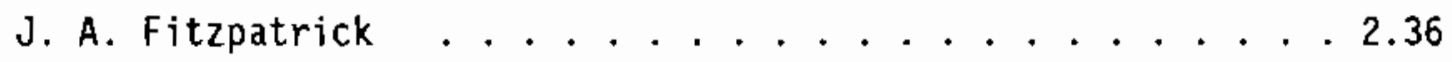

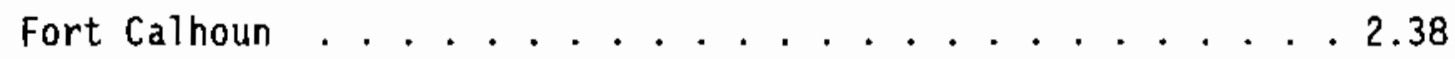

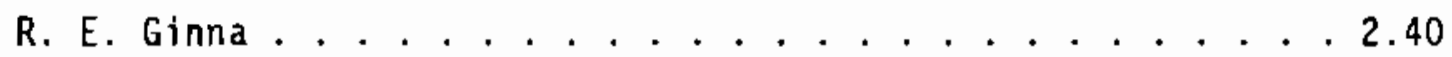

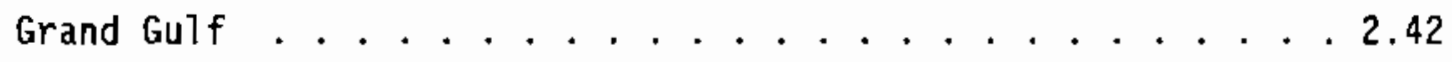

Haddam Neck . . . . . . . . . . . . . . 2.44

Edwin I. Hatch $1 \& 2 \ldots \ldots . \ldots . \ldots . \ldots 2 . \ldots$

Indian Point $1,2,83 \ldots \ldots \ldots \ldots$

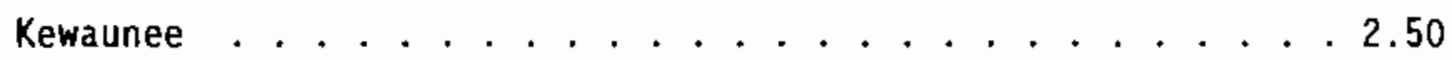

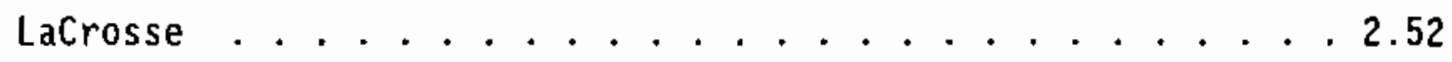

LaSalle $1 \& 2 \ldots \ldots . \ldots \ldots . \ldots . \ldots . \ldots . \ldots$

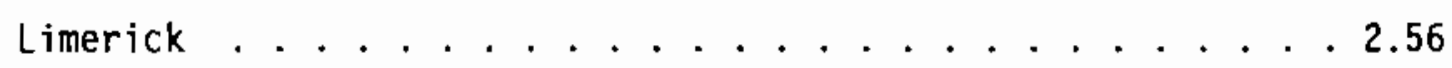

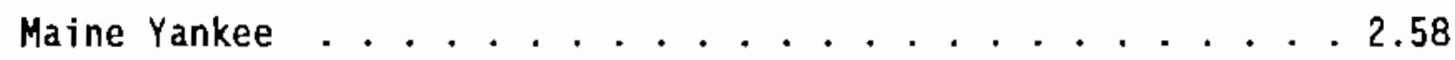

McGuire $1 \& 2 \ldots \ldots . \ldots . \ldots . \ldots 2 . \ldots$

Millstone $1 \& 2 \ldots \ldots . \ldots \ldots . \ldots . \ldots 2 . \ldots \ldots$

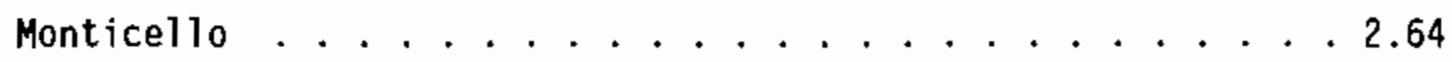

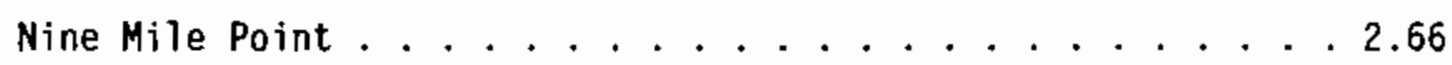

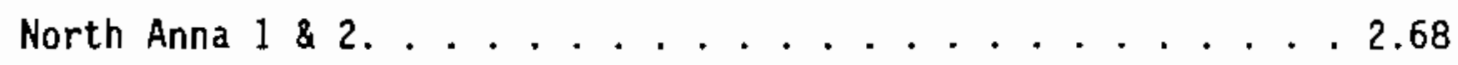

Oconee $1,2 \& 3 \ldots \ldots \ldots \ldots$

oyster creek . . . . . . . . . . . . . . . 2.72

Palisades . . . . . . . . . . . . . . . . . . . . . . . . . .

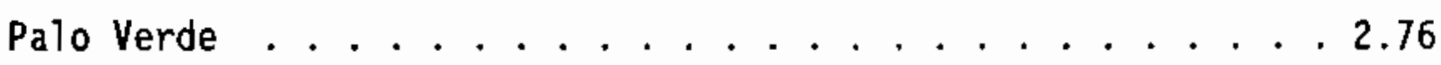


Peach Bottom $2 \& 3 \ldots \ldots \ldots \ldots . \ldots . \ldots . \ldots . \ldots$ Pilgrim ..................... 2.80

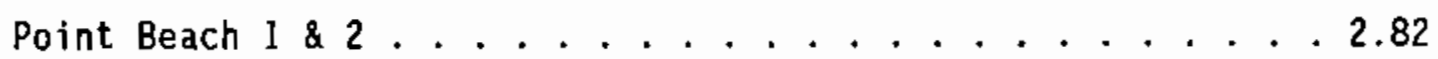

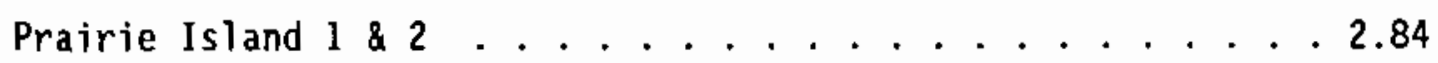
Quad Cities $1 \& 2 \ldots \ldots \ldots . \ldots \ldots$

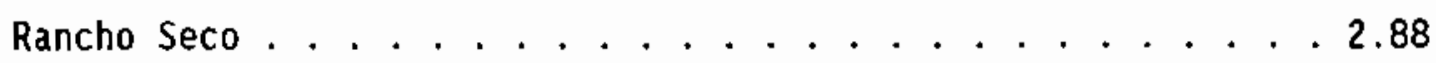
H. B. Robinson . . . . . . . . . . . 2.90 St. Lucie $1 \& 2 \ldots \ldots . \ldots . \ldots . \ldots . \ldots 2$

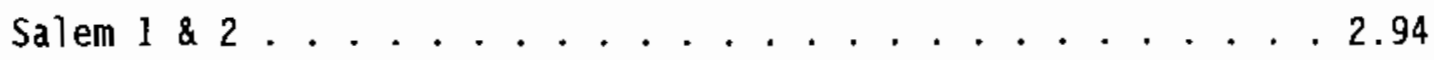

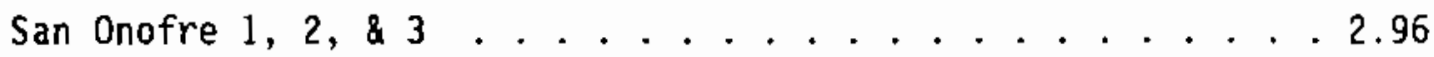
Sequoyah $1 \& 2 \ldots \ldots \ldots . \ldots \ldots$ Summer ............................... . . . . . Surry $1 \& 2 \ldots \ldots . \ldots \ldots . \ldots . \ldots . \ldots 2 . \ldots \ldots$

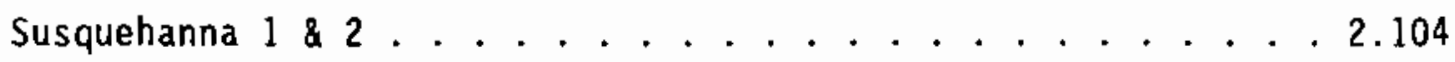
Three Mile Island $1 \& 2 \ldots \ldots . \ldots . \ldots . \ldots . \ldots 606$

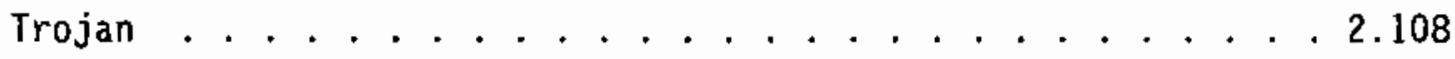
Turkey Point $3 \& 4 \ldots \ldots . \ldots . \ldots . \ldots . \ldots 110$

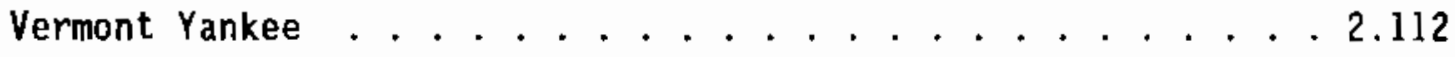
Waterford ............................ 2.114

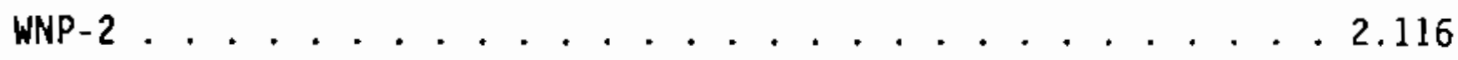
Wolf Creek . . . . . . . . . . . . . 2.118 Yankee Rowe . . . . . . . . . . . . . . 2. 2.120 Zion $1 \& 2 \ldots \ldots . \ldots . \ldots . \ldots . \ldots 2 . \ldots \ldots$ 


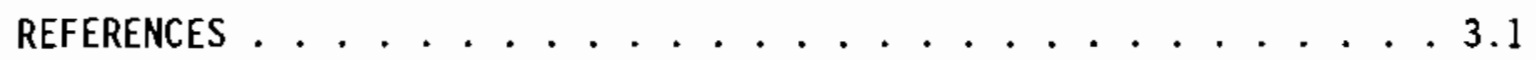
APPENDIX ............................. A-1 


\section{ACKNOWLEDGMENTS}

The author greatly appreciates the development of the population distributions around each site from census data by Anton A. Sinisgalli, A. R. Brauner, and Donald P. Cleary of the U.S. Nuclear Regulatory Commission. The author also gratefully acknowledges the contributions and suggestions by Richard $A$. Hartfield and Kazimieras Campe, also of the U.S. Nuclear Regulatory Commission. 



\section{INTRODUCTION}

Most commercial nuclear power reactors release small amounts of radioactive materials to the environment during normal operation. Because of these releases, concern was expressed about the magnitude of the collective dose received by the general population residing around these nuclear power plants. In response to this concern, the Pacific Northwest Laboratory (PNL) (a) contracted with the Nuclear Regulatory Commission (NRC) to undertake a series of studies to estimate radiation dose commitments produced by radionuclide releases from commercial light-water power reactors during the years 1975 through 1985 (see previous reports in this series, p. ii). This document is a continuation of these studies and considers the doses from releases during 1985. In this study, as in those previous, we estimated the collective (population) dose commitment (b) from both the liquid and gaseous releases to four age groups making up the population residing in the region of the site: infant ( 0 to $1 \mathrm{yr}$ ), child (1 to $11 \mathrm{yr}$ ), teen-ager (11 to $17 \mathrm{yr}$ ), and adult (17 $\mathrm{yr}$ and older).

The particular organs of reference in this study are listed in Table 1. The major pathways by which radionuclides travel from the reactor to the individual receptors are shown in Table 2. Other possible liquid pathways such as direct exposure from waterborne activities (swimming, boating, shoreline recreation) and internal exposure through ingestion of food produced using contaminated irrigation water were not included; we have found from past experience that the doses from these pathways are generally much smaller than the doses from the pathways considered in this study.

The "source terms" used to estimate dose commitments produced from each site were the annual measured releases of radioactive materials as reported to the NRC by the plant operators and subsequently published in an NRC public document (Tichler, et al. 1988).

The regional population for which we estimated dose commitments included those persons estimated to be living in a region between 2 and 80 $\mathrm{km}$ around the reactor sites during 1985.

(a) Operated by Battelle Memorial Institute for the Department of Energy.

(b) As used in this report, dose commitment describes the total-body dose equivalent in rem ( 1 rem $=0.01$ sievert) received over 50 years from intake during the year in which radioactive materials were released into the environment from the plants. 
TABLE 1. Organs Considered in This Study

Organs Affected by
Airborne Releases

Total body

Thyroid

Bone

GI tract

Liver

Lung
Organs Affected by

Waterborne Releases

Total body

Thyroid

Bone

GI tract

Liver

IABLE 2. Pathways Considered in This Study by which Radionuclides Travel from Reactors to Persons

Pathways for Airborne Releases

Air submersion

Contaminated ground

Inhalation

Ingestion of food crops

and animal products
Pathways for Waterborne Releases

Ingestion of drinking water Ingestion of fish and invertebrates

Population distributions were supplied by the NRC's office of Nuclear Reactor Regulation. Atmospheric transport factors (annual average dilution and annual average deposition) were calculated for the region around each site using appropriate meteorological data supplied by the NRC or the utility. To calculate the doses, we used models approved by the NRC. We incorporated these models into two small computer codes to expedite the dose calculations involved for each site.

Site-specific parameters other than releases, meteorology, and population were obtained from environmental statements [both final (FES) and draft (DES)] for the various reactors when available (Table 3). Such parameter values include the total population drinking contaminated water, river flow, dilution flow from the reactors (for sites not on rivers), fish and invertebrate harvest for region, and dilution factors for drinking water and aquatic foods. In those cases in which site-specific data are not readily available and the particular pathway is not expected to result in a large dose, conservative assumptions have been used to estimate doses. The use of more realistic data should result in lower dose estimates in some cases. 


\section{IABLE 3. Environmental Statements for Power Plants Included in This Study}

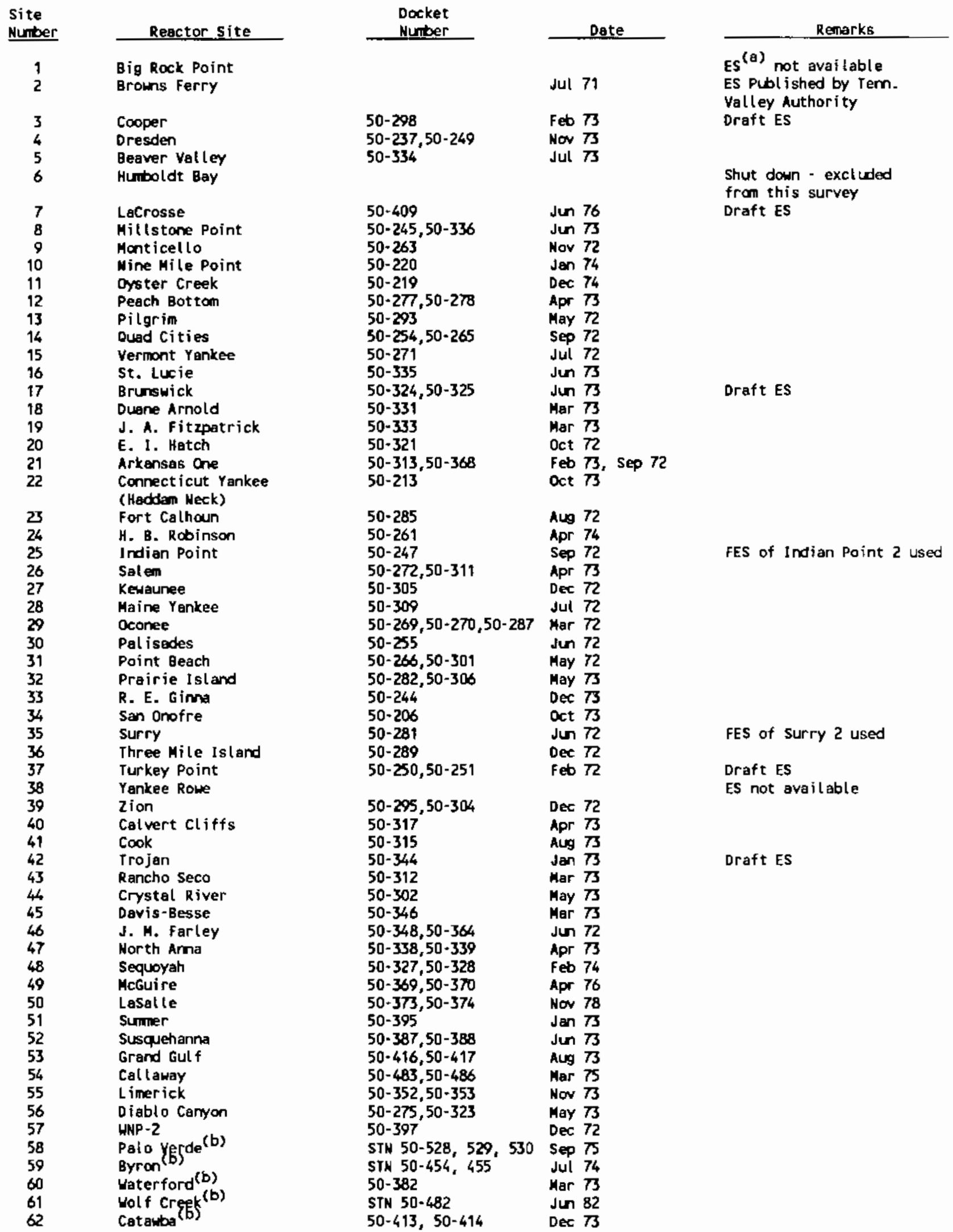


The reactors included in this study, their type, licensed thermal power rating and net electrical output for 1985 are 1 isted in Table 4. Populations at risk and the dose commitments derived in the study are also shown in this table.

\section{Site-Dependent Parameters}

In the Site Summaries section, the location (including latitude and longitude) for each reactor site and the estimated 1985 population within 2 to $80 \mathrm{~km}$ around each site is given. In addition, the locations of major metropolitan centers within $80 \mathrm{~km}$ are listed along with their 1985 extrapolated populations. The populations of the Standard Metropolitan Statistical Areas (SMSA) are given where applicable. Next, the site-specific data pertinent to the airborne pathways are specified. The average production rates of vegetable crops and animal products are given for the area within an $80-\mathrm{km}$ radius based upon the statewide average. This production has been reduced for sites on lakes and seacoasts to account for the presence of the body of water. An animal grazing factor is estimated for each site location. This factor accounts for the fraction of the year during which grazing animals such as milk cows and beef cattle graze on fresh pasture in the region around the site. After average production rates are given, the period of record and the percent data recovery of the meteorological data used in calculating diffusion factors are indicated.

Various site-dependent factors associated with the waterborne pathways are presented next. For lake and ocean sites, we used the average dilution of plant effluents for the year 1985 specified by Tichler, et a1. (1988). For river sites, the average annual river flow is tabulated. This flow was used in place of a dilution flow from the plant to account for dilutions of liquid releases at the locations of probable intake of drinking water and aquatic food catch. Any exceptions to this scheme have been footnoted. Next is shown the estimated 1985 population utilizing drinking water drawn from supplies containing diluted effluents from the site. These are shown with an estimated dilution factor where applicable. Fish and invertebrate catch data taken from the respective plant environmental statement, when available (see Table 3), are listed next, along with estimated dilution factors for the lake and ocean sites. When site-specific fish and invertebrate catch data were not available, the generic consumption rates were used for the particular site. Sites on salt water were assumed to contribute no dose from drinking water. Any invertebrate catch from fresh water was not considered. 


\section{TABLE 4. Reactor Characteristics and Population Total-Body Dose Commitments, 1985}

\begin{tabular}{|c|c|c|c|c|c|c|c|c|c|}
\hline \multirow[b]{2}{*}{ site } & \multirow[b]{2}{*}{ unlt } & \multirow[b]{2}{*}{ Iype } & \multirow{2}{*}{$\begin{array}{c}\text { Licensed } \\
\text { rheraal } \\
\text { Power } \\
\text { (MW ) } \\
\end{array}$} & \multirow{2}{*}{$\begin{array}{c}\text { Electric } \\
\text { Energy } \\
\text { ceneration } \\
1995 \text { ias } \\
\text { (Tw.hr) }\end{array}$} & \multicolumn{3}{|c|}{$\begin{array}{c}\text { Populatlon Dose comil tment } \\
\text { (person-ren) }\end{array}$} & \multirow{2}{*}{$\begin{array}{l}\text { Population } \\
\text { at Risk- }\end{array}$} & \multirow{2}{*}{$\begin{array}{c}\text { Average } \\
\text { tndividual } \\
\text { Total-body } \\
\text { Dose } \\
\text { Comaitment } \\
\text { (mirem) } \\
\end{array}$} \\
\hline & & & & & liquid & $A 1 r$ & Total & & \\
\hline $\begin{array}{l}\text { Arkansas one } \\
\text { Arkansas one }\end{array}$ & $2_{\text {TOTAL }}^{1}$ & $\begin{array}{l}\text { PWR } \\
\text { PWR }\end{array}$ & $\begin{array}{l}2568 \\
2815 \\
5383\end{array}$ & $\begin{array}{l}5.19 \\
4.70 \\
9.09\end{array}$ & 1.9 & 0.11 & 2.0 & $1.9 \mathrm{E} 5$ & $1.1 E-2$ \\
\hline Eeaver valley & 1 & PWR & 2652 & 5.90 & 0.018 & 0.038 & 0.056 & $3.6 \mathrm{E} 6$ & $1.6 \mathrm{E}-5$ \\
\hline Big Rack Point (b) & 1 & BWR & 240 & 0.362 & 0.66 & 2.6 & 3.3 & $17 \mathrm{ES}$ & $2.0 \mathrm{E}-2$ \\
\hline $\begin{array}{l}\text { Brown gerty } \\
\text { Browns ferry }\end{array}$ & $\begin{array}{l}1 \\
2 \\
3 \\
\text { TOTAL }\end{array}$ & $\begin{array}{l}\text { BWR } \\
\text { BWR } \\
\text { GWR }\end{array}$ & $\begin{array}{l}3293 \\
3293 \\
3293 \\
9879\end{array}$ & $\begin{array}{l}1.54 \\
0 \\
1.47 \\
3.01\end{array}$ & 3.4 & 4.5 & 7.9 & $7.4 € 5$ & 1. $1 E-2$ \\
\hline $\begin{array}{l}\text { Brunswick } \\
\text { Brunswlck }\end{array}$ & ${ }^{1}$ TOTAL & $\begin{array}{l}\text { BWR } \\
\text { BWR }\end{array}$ & $\begin{array}{l}2436 \\
2436 \\
4872\end{array}$ & $\begin{array}{l}1.91 \\
5.02 \\
6.93\end{array}$ & 0.0015 & 0.18 & 0 is & 2.365 & $7.9 E-4$ \\
\hline Eyron & 1 & $\mathbf{P} w \boldsymbol{R}$ & 3411 & 1.01 & 0.23 & $\mathbf{0 . 0 2}$ & 0.27 & 9.365 & $2.9 E-4$ \\
\hline callaway & 1 & PER & 3411 & 0.05 & 0 & 0.011 & 0.011 & $3.7 E 5$ & $3.0 \mathrm{E}-5$ \\
\hline $\begin{array}{l}\text { calvert clifts } \\
\text { calvert clifts }\end{array}$ & $\begin{array}{l}1 \\
2 \\
\text { TOTAL }\end{array}$ & $\begin{array}{l}\text { PWR } \\
\text { PWR }\end{array}$ & $\begin{array}{l}2700 \\
2700 \\
5400\end{array}$ & $\begin{array}{l}1.36 \\
5.61 \\
9.97\end{array}$ & 0.68 & 0.14 & 0.12 & 2.7E6 & 2. DE-4 \\
\hline Catawba & 1 & PWR & 3411 & 3.44 & 0.72 & 0.02 & 0.74 & t. $5 \mathrm{ES}^{-}$ & $4.8 E-4$ \\
\hline $\begin{array}{l}\text { cook } \\
\text { cook }\end{array}$ & ${ }^{1}$ TOTAL & $\begin{array}{l}\text { PWR } \\
\text { PWR }\end{array}$ & $\begin{array}{l}3250 \\
3391 \\
6641\end{array}$ & $\begin{array}{l}2.12 \\
5.68 \\
7.80\end{array}$ & 0.47 & 0.071 & 0.54 & 1. $1 \mathrm{E} 6$ & 4. $B E-4$ \\
\hline cooper & 1 & BWR & 2381 & 1.07 & 0.056 & 0.013 & 0.069 & $1.7 \mathrm{ES}$ & $4.0 E-4$ \\
\hline Crystal River & 3 & Pwr & 2452 & 2.85 & 4.3 & 0.12 & 4.4 & $4.3 \mathrm{E} 5$ & $1.0 \mathrm{E}-\mathbf{2}$ \\
\hline Davis-Besse & 1 & PWR & 2772 & 1.94 & 0.49 & 0.0061 & 0.50 & 1.896 & $2.3 E-4$ \\
\hline $\begin{array}{l}\text { Diablo canyon } \\
\text { Diablo canyon }\end{array}$ & $2_{\text {TOTAL }}^{1}$ & $\begin{array}{l}\text { PWR } \\
\text { PWR }\end{array}$ & $\begin{array}{l}3338 \\
3411\end{array}$ & $\begin{array}{l}5.23 \\
0.54 \\
5.77\end{array}$ & 0.0036 & a. 0070 & 0.011 & 2.915 & $3.7 E-5$ \\
\hline oresden & $\begin{array}{l}1 \\
2 \\
3 \\
\text { TOTAL }\end{array}$ & $\begin{array}{l}\text { BWR } \\
\text { BWR } \\
\text { BWR }\end{array}$ & $\begin{array}{r}700 \\
2527 \\
2527 \\
5754\end{array}$ & $\begin{array}{l}0 \\
3.09 \\
4.39 \\
7.48\end{array}$ & $\mathbf{0}$ & 0.96 & 0.96 & 6.416 & 1. $5 E-4$ \\
\hline Duane arnold & 1 & GWR & 1638 & 1.94 & 0.0010 & 0.023 & 0.024 & $6.0 \mathrm{E} 5$ & $4,0 E-5$ \\
\hline $\begin{array}{l}\text { J. M. Farley } \\
\text { J. M Farley }\end{array}$ & ${ }^{1}$ rotAL & $\begin{array}{l}\text { PWR } \\
\text { PWR }\end{array}$ & $\begin{array}{l}2652 \\
2652 \\
5304\end{array}$ & $\begin{array}{r}5.87 \\
5.47 \\
11.34\end{array}$ & 0.0089 & 0.13 & 0.14 & 3 TE 5 & $3.8 F-4$ \\
\hline 1. A. Fltzpatrick & 1 & $8 w R$ & 2436 & 4.17 & 0.025 & 0.38 & 0.40 & B. $5 k 5$ & $4.7 E-4$ \\
\hline fort calhoun & 1 & PWR & 1420 & 307 & 0.23 & 0.014 & 0.24 & 7.665 & 3. $2 E-4$ \\
\hline R E. Ginna & 1 & PwR & 1520 & 362 & 0.28 & 0.034 & 0.31 & $1.26 \mathbf{6}$ & $2.6 E-4$ \\
\hline Grand Gull & 1 & BwR & 3833 & 2.65 & 0.0001 & 0.0015 & 0.0016 & 3.355 & 4. $9 E-6$ \\
\hline Haddan Neck & 1 & PWR & 1825 & 4.64 & 0.056 & 0.55 & 0.61 & 3.556 & $1.8 E-4$ \\
\hline $\begin{array}{l}\text { E. I. Hatch } \\
\text { \&. I. Hatch }\end{array}$ & $2_{\text {TOTAL }}^{1}$ & $\begin{array}{l}\text { BWR } \\
\text { BWR }\end{array}$ & $\begin{array}{l}2436 \\
2436 \\
4877\end{array}$ & $\begin{array}{r}4.76 \\
5.38 \\
10.14\end{array}$ & 3.3 & 0.086 & 3.4 & 3. $3 \mathrm{E} 5$ & $1.0 \mathrm{E}-2$ \\
\hline Indan point & $\begin{array}{l}1 \\
2 \\
3 \\
\text { TOT AL }\end{array}$ & $\begin{array}{l}\text { PWR } \\
\text { PWR } \\
\text { PWR }\end{array}$ & $\begin{array}{r}815 \\
2758 \\
2760 \\
6133\end{array}$ & $\begin{array}{r}0 \\
6.67 \\
4.73 \\
11.40\end{array}$ & 0.57 & 2.2 & 2.8 & $1.6 \mathrm{E7}$ & 1. $8 E-4$ \\
\hline Kewaunee & 1 & PWR & 1650 & 3.70 & 1.2 & 0.0027 & 1.2 & $0.3 \mathrm{ES}$ & $1.9\{-3$ \\
\hline Lacrosse $(b)$ & 1 & $8 * R$ & 165 & 0.323 & 0.52 & 53 & 1.0 & $3.5 E S$ & $3.0 \mathrm{E}-3$ \\
\hline $\begin{array}{l}\text { Lasalle } \\
\text { lasalle }\end{array}$ & ${ }^{1}$ TOThL & $\begin{array}{l}\text { EWR } \\
\text { BWR }\end{array}$ & $\begin{array}{l}3323 \\
3293 \\
6616\end{array}$ & $\begin{array}{l}4.89 \\
3.43 \\
\text { B. } 24\end{array}$ & 0 & 0.073 & 0.073 & $1.06 \mathbf{6}$ & $7.0 \mathrm{E}-5$ \\
\hline 1. imer ick & & HWR & 3293 & 1.14 & 0.070 & 0 & 0.078 & $68 E 6$ & $1 \cdot 2 E-5$ \\
\hline matne rankee & 1 & $\mathrm{PWR}$ & 2440 & 5.35 & 0.0003 & 0.011 & 0.011 & 6. $1 E 5$ & $1.8 \mathrm{E}=5$ \\
\hline $\begin{array}{l}\text { meculré } \\
\text { mcGuire }\end{array}$ & $\sum^{1}$ TOTAL & $\begin{array}{l}\text { PWR } \\
\text { PWR }\end{array}$ & $\begin{array}{l}3411 \\
3411 \\
6822\end{array}$ & $\begin{array}{r}5.78 \\
5.60 \\
12.38\end{array}$ & 20. & 0.16 & 20 & $1.7 E 6$ & $1.2 \mathrm{E}-2$ \\
\hline $\begin{array}{l}\text { millstone } \\
\text { mIIIstone }\end{array}$ & $\begin{array}{l}1 \\
2 \\
\operatorname{LOTAL}\end{array}$ & 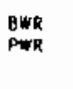 & $\begin{array}{l}2011 \\
2560 \\
4571\end{array}$ & $\begin{array}{l}.59 \\
3.50 \\
9.09\end{array}$ & 0.053 & 0.21 & o. 26 & $2.6 \mathrm{E6}$ & $1.0 E-4$ \\
\hline monticedlo & 1 & $6 \% R$ & 1670 & 429 & 0 & 0.11 & 0.14 & $2.2 E 6$ & $6.4 t-5$ \\
\hline Nine wile Polnt & 1 & GWR & 1850 & 4.93 & 0 & 0.018 & 0.018 & 0.565 & $2.1 k-5$ \\
\hline $\begin{array}{l}\text { North Anna } \\
\text { North Anna }\end{array}$ & $\begin{array}{ll}1 & \\
2 & T O T A L\end{array}$ & $\begin{array}{l}\text { PWR } \\
\text { PWR }\end{array}$ & $\begin{array}{l}2775 \\
2775 \\
5550\end{array}$ & $\begin{array}{r}5.80 \\
6.81 \\
12.60\end{array}$ & 27. & 0.080 & 27. & $1.1 E 6$ & $2.5 E-2$ \\
\hline
\end{tabular}




\section{IABLE 4. (Contd)}

\begin{tabular}{|c|c|c|c|c|c|c|c|c|c|}
\hline \multirow[b]{2}{*}{ site } & \multirow[b]{2}{*}{ Unlt } & \multirow[b]{2}{*}{ Type } & \multirow{2}{*}{$\begin{array}{c}\text { Licensed } \\
\text { Thermal } \\
\text { Power } \\
\text { (aw) } \\
\end{array}$} & \multirow{2}{*}{$\begin{array}{c}\text { Electric } \\
\text { Energy } \\
\text { ceneration } \\
1985 \text { (a) } \\
\text { (Tw.hr) }\end{array}$} & \multicolumn{3}{|c|}{$\begin{array}{c}\text { Population oose com l tment } \\
\text { (person-rean }\end{array}$} & \multirow{2}{*}{$\begin{array}{l}\text { Population } \\
\text { at Risk }\end{array}$} & \multirow[t]{2}{*}{$\begin{array}{c}\text { Average } \\
\text { Indivldual } \\
\text { Total-body } \\
\text { Dose } \\
\text { Comitment } \\
\text { (nren) }\end{array}$} \\
\hline & & & & & llould & AlP & Tota: & & \\
\hline $\begin{array}{l}\text { oconee } \\
\text { oconee }\end{array}$ & $\begin{array}{l}1 \\
2 \\
3 \\
\end{array}$ TOTAL & $\begin{array}{l}\text { PwR } \\
\text { PwR } \\
\text { PwR }\end{array}$ & $\begin{array}{l}2568 \\
2568 \\
2568 \\
7704\end{array}$ & $\begin{array}{r}7.07 \\
5.06 \\
4.06 \\
15.99\end{array}$ & 6.4 & 0.50 & 6.9 & $9.6 \mathrm{E5}$ & $7.2 E-3$ \\
\hline oyster Creek & 1 & $\mathrm{BWR}$ & 1930 & 3.75 & 0 & 73 & 73 & $3.6 \mathrm{Eg}$ & $2.0 E-2$ \\
\hline Pal|sades & 1 & PWR & 25.30 & 5. 30 & 0.27 & 0.035 & 0.30 & 1. DE 6 & 2. $9 E-4$ \\
\hline Palo verde & 1 & Pwr & 3800 & 1.13 & 0 & 0.0033 & 0.0033 & $1.1 E 6$ & 2. $9 E-6$ \\
\hline $\begin{array}{l}\text { Peach Bottom } \\
\text { Peach 8otton }\end{array}$ & $\begin{array}{l}2 \\
3 \\
\text { TOTAL }\end{array}$ & $\begin{array}{l}\text { BWR } \\
\text { BWR }\end{array}$ & $\begin{array}{l}3293 \\
3293 \\
6586\end{array}$ & $\begin{array}{l}2.33 \\
3.28 \\
5.61\end{array}$ & 1. 2 & 15. & 16 & 4. 366 & $3 . \mathrm{aE}-3$ \\
\hline Pllgrim & 1 & Bwk & 1998 & 4.95 & 0.016 & 0.54 & 0.56 & $4.4 E 6$ & $1.3 E-4$ \\
\hline $\begin{array}{l}\text { point Beach } \\
\text { polnt Beach }\end{array}$ & 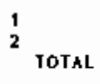 & $\begin{array}{l}\text { PWR } \\
\text { PWR }\end{array}$ & $\begin{array}{l}1518 \\
1518 \\
3036\end{array}$ & $\begin{array}{l}3.33 \\
3.60 \\
6.95\end{array}$ & 0.075 & 0.020 & 0.095 & 6. $2 \mathrm{ES}$ & $1,5 E-4$ \\
\hline $\begin{array}{l}\text { Praifle island } \\
\text { pralile island }\end{array}$ & $2_{\text {TOTAL }}^{1}$ & $\begin{array}{l}\text { PWR } \\
\text { PWR }\end{array}$ & $\begin{array}{l}1650 \\
1650 \\
3300\end{array}$ & $\begin{array}{l}3.68 \\
3.61 \\
7.29\end{array}$ & 00059 & 0.038 & 0.044 & $2.2 \mathrm{EG}$ & $2.0 \varepsilon \cdot 5$ \\
\hline $\begin{array}{l}\text { Quad cities } \\
\text { Quad cittes }\end{array}$ & $\begin{array}{ll}1 & \\
2 & \\
& \text { TOTAL }\end{array}$ & $\begin{array}{l}\text { ewR } \\
\text { ewR }\end{array}$ & $\begin{array}{l}2511 \\
2511 \\
5022\end{array}$ & $\begin{array}{r}6.07 \\
4.56 \\
10.63\end{array}$ & 5.5 & 0.19 & 5.7 & $7.2 \mathrm{ES}$ & $7.9 E-3$ \\
\hline Rancho seco & 1 & PWR & 2772 & 1.96 & 0.072 & 0.18 & 0.25 & 2.0 & $1.3 E-4$ \\
\hline H. B. Rooinson & 2 & PwR & 2200 & 5.24 & 0.16 & 0.054 & 0.21 & $7.1 E 5$ & 3. $\mathrm{OE}-4$ \\
\hline $\begin{array}{l}\text { St. Lucie } \\
\text { St. Lucie }\end{array}$ & $\begin{array}{l}1 \\
2 \\
\text { TOTAL }\end{array}$ & $\begin{array}{l}\text { PWR } \\
\text { PWR }\end{array}$ & $\begin{array}{l}2560 \\
2700 \\
5260\end{array}$ & $\begin{array}{r}5.87 \\
6.11 \\
11.98\end{array}$ & 0.012 & 1.6 & 1.6 & 6.715 & $2.4 \mathrm{E}-3$ \\
\hline $\begin{array}{l}\text { 5alem } \\
\text { salem }\end{array}$ & ${ }^{1}$ TOTAL & $\begin{array}{l}\text { PwR } \\
\text { PwR }\end{array}$ & $\begin{array}{l}3338 \\
3338 \\
6676\end{array}$ & $\begin{array}{r}9.01 \\
5.02 \\
14.03\end{array}$ & $0.4 \mathrm{a}$ & 0.085 & 0.56 & $4.8 E 6$ & 1. $2 \mathrm{E}-4$ \\
\hline $\begin{array}{l}\text { san onolre } \\
\text { san onof se }\end{array}$ & $\begin{array}{ll}1 & \\
2 & \\
3 & \\
& \text { TOTAL }\end{array}$ & $\begin{array}{l}P W R \\
P W R \\
P W R\end{array}$ & $\begin{array}{l}1347 \\
34+0 \\
3390 \\
8147\end{array}$ & $\begin{array}{r}2.46 \\
5.15 \\
3.71 \\
11.32\end{array}$ & 046 & 1.5 & 2.0 & $5.2 E 6$ & $3.8 E-4$ \\
\hline $\begin{array}{l}\text { Sequoyah } \\
\text { sequoyah }\end{array}$ & $2^{1}$ TOTAL & $\begin{array}{l}\text { PWR } \\
\text { PWR }\end{array}$ & $\begin{array}{l}2815 \\
2815 \\
5630\end{array}$ & $\begin{array}{l}4.06 \\
5.61 \\
9.67\end{array}$ & 0.79 & 0.27 & 11 & B. $6 \mathrm{ES}$ & 1. $2 \mathrm{E}-3$ \\
\hline summer & 1 & PWR & 2775 & 5.23 & 0.56 & $0.0 \times 25$ & 0.56 & $0.7 \mathrm{ES}$ & $6.4 E-4$ \\
\hline $\begin{array}{l}\text { Surry } \\
\text { surry }\end{array}$ & $\begin{array}{ll}1 & \\
2 & \\
& \text { IOTAL }\end{array}$ & $\begin{array}{l}\text { PwR } \\
P w R\end{array}$ & $\begin{array}{l}2441 \\
244 t \\
4882\end{array}$ & $\begin{array}{l}5.62 \\
4.07 \\
9.69\end{array}$ & 1.5 & 0.16 & 1.6 & $1.0 \mathrm{E} 6$ & B. $9 E-4$ \\
\hline $\begin{array}{l}\text { Susquehanna } \\
\text { susquehanna }\end{array}$ & $\begin{array}{ll}1 & \\
2 & \text { TOTAL }\end{array}$ & $\begin{array}{l}\text { BWR } \\
\text { BWR } \\
\text { BWR }\end{array}$ & $\begin{array}{l}3293 \\
3293 \\
6586\end{array}$ & $\begin{array}{r}5.26 \\
6.95 \\
12.21\end{array}$ & 0.011 & $0.0 \subseteq 5$ & 0. 11 & 1. $5 E 6$ & $7.16-5$ \\
\hline $\begin{array}{l}\text { Three mile Island } \\
\text { Three mile Island }\end{array}$ & ${ }^{1} \operatorname{rotal}$ & $\underset{\substack{p W R \\
\text { PWR }}}{\operatorname{PWR}}$ & $\begin{array}{l}2535 \\
2772 \\
5307\end{array}$ & $\begin{array}{l}0.812 \\
0 \\
0.812\end{array}$ & 0.012 & 0045 & 0.057 & 2.166 & $2.7 E \cdot 5$ \\
\hline Trojan & 1 & PWR & 3411 & 691 & 0.041 & $0 \quad 0 \div 5$ & 0.096 & $1.4 E 6$ & $6.7 E-5$ \\
\hline $\begin{array}{l}\text { Turkey point } \\
\text { Turkey polnt }\end{array}$ & 3 TOTAL & $\begin{array}{l}\text { PWR } \\
\text { PWR }\end{array}$ & $\begin{array}{l}2200 \\
2200 \\
4100\end{array}$ & $\begin{array}{l}3.41 \\
5.18 \\
3.59\end{array}$ & 00081 & $0.1 \leq$ & o. 14 & $2.8 \mathrm{Eg}$ & $5.06 \cdot 5$ \\
\hline Vermont rankee & 1 & BWR & 1593 & 3.00 & 0 & 0.11 & 0.10 & $1.4 E 6$ & $7 \cdot 2 E \cdot 5$ \\
\hline waterford & 3 & PWR & 3390 & 1.81 & 0.0032 & $0.4:$ & 0.47 & $1.9 E 6$ & $2.5 E-4$ \\
\hline WNP-2 & & BWR & 3323 & 5.18 & 0.0005 & 0.017 & 0.018 & $2.6 E 5$ & $6.6 E-5$ \\
\hline wolf creek & 1 & Pwe & 3411 & 2.94 & 0.081 & $0.0: 9$ & 0.11 & 1.815 & $6.0 E \cdot 4$ \\
\hline Yankee Rowe & 1 & $\rho * R$ & 600 & 1.00 & 0.25 & 0.12 & 0.39 & $1.6 E 6$ & $2 \cdot 4 E-4$ \\
\hline $\begin{array}{l}\text { zion } \\
\text { zion }\end{array}$ & $\begin{array}{l}1 \\
2 \\
\text { TOTAL }\end{array}$ & $\begin{array}{l}\text { PWR } \\
\text { PWR }\end{array}$ & $\begin{array}{l}3250 \\
3250 \\
6500\end{array}$ & $\begin{array}{l}\text { 4. } 81 \\
5.11 \\
9.92 \\
\end{array}$ & 6.5 & 0.34 & 7.2 & 7.256 & $1.0[-4$ \\
\hline TOTAL FOR ALL SITFS & & & & 373.77 & $\$ 1$. & 110. & 200 & 1.250 &.- \\
\hline Arithmetic Hean & & & & 6.1 & 1.5 & 1.6 & 3.3 & 1.916 & $2.6 \mathrm{E} \cdot 3$ \\
\hline Geometrit mean & & & & 3.1 & 0.13 & 0.10 & 0.37 & $1.1 \mathrm{E} 6$ & $3 \cdot 3 t-4$ \\
\hline
\end{tabular}

(a) 1 Tw-hr $=3$. ot 15 joules.

(b) Does not have charcoal delay beds In the gaseous elfluent line from air ejector 


\section{RESULTS}

This report consists of a summary of values used for site-specific parameters at each site, as explained above, and the results of population dose commitment calculations. The population dose commitments are presented in two tables facing the page summarizing site-specific parameters for that site. These tables include both waterborne and airborne pathway dose commitments for the several organs of reference for each age group investigated. They also include the dose to the whole population, which includes all age groups. The airborne population dose commitments for each of 160 segments(a) partitioning the region around the site were divided by the population residing within that segment to derive an average individual dose for that segment. These doses are summarized as a histogram showing percent of the population receiving a given dose level for each site. The fractional population dose from the waterborne pathway was not determined in this manner, because the NRC does not at present take into account the location of individuals exposed via this pathway, except those exposed through ingestion of drinking water.

Population dose commitments estimated for both the waterborne pathways and airborne pathways varied widely over the 61 sites studied. The total dose commitments (from both pathways) varied from a high of 73 to a low of 0.011 person-rem for plants in operation during the whole year. The arithmetic mean for the total dose from liquid pathways (1.8 person-rem) and airborne pathways (1.5 person-rem) was 3 person-rem (see Table 4).

As in past years, most of the plants accounted for less than a person-rem to their surrounding population from $p l a n t$ releases of radionuclides into liquid effluent streams. Only two sites had waterborne releases that resulted in population doses exceeding 10 person-rem. Those sites were North Anna (27 person-rem) and McGuire (20 person-rem). These waterborne doses were due almost totally to radioactive cesium and tritium (McGuire).

The doses from radionuclide releases from plant vents and/or stacks into the atmosphere also accounted for less than a person-rem for most sites. Only two sites had airborne releases resulting in 10 or more person-rem. These sites were Oyster Creek (73 person-rem) and Peach Bottom (15 person-rem). These airborne doses were the result of xenons (133 and 135) and krypton-88 and its daughters.

The total population dose commitments from all sites for 1985 were estimated to be 91 person-rem via waterborne pathways and 110 person-rem via airborne pathways (Table 4).

(a) See Appendix for definition of segments. 
Figure 1 is a histogram for all 61 sites taken together. We can see from this plot that about $78 \%$ of the total population at risk (110 million) would each receive a dose commitment of between $1 \times 10^{-6}$ and $3 \times 10^{-4}$ mrem. We can see further that about $3 \%$ receive a dose which is less than $3 \times 10^{-6}$ mrem. Although not discernible from the plot, $0.4 \%$ received a dose of between 0.03 and $1 \mathrm{mrem}$. However, no attempt was made in this study to estimate the maximum dose commitment received by any one individual from the radionuclides released at any of the sites.

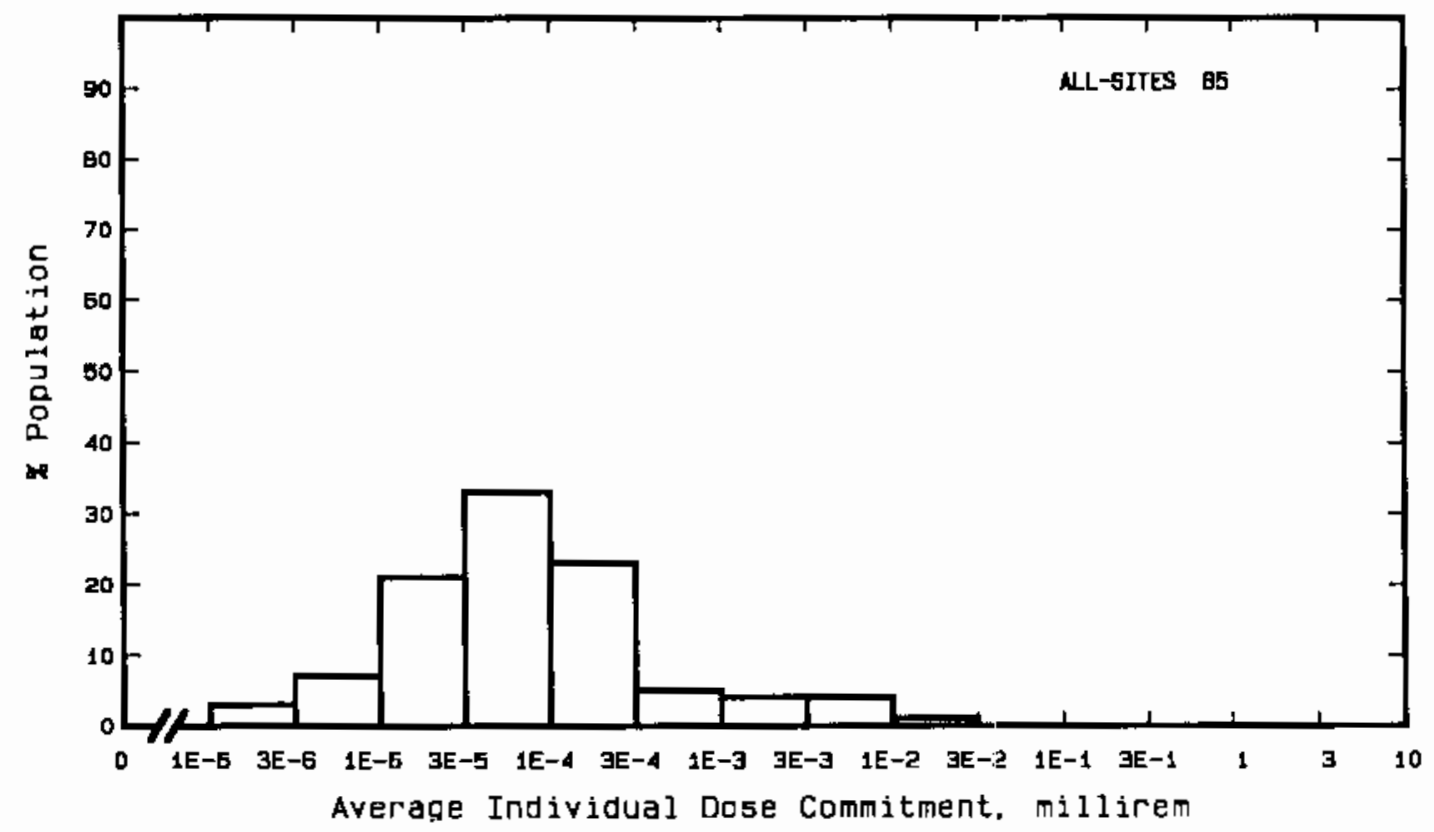

FIGURE 1. Fraction of Total Population Receiving Various Average Individual Total-Body Dose Commitments for All Sites

Figure 2 shows graphically in "Box-and-Whisker Plots" the wide range of the airborne population dose commitments for the reactor sites. The median, upper and lower quartiles and upper and lower octiles for the distribution of doses calculated for each of 160 segments are indicated for each site. The upper and lower octiles are shown as the horizontal bars on the "whiskers;" the upper and lower quartiles are indicated as the top and bottom of the "box." The median is indicated as the horizontal line inside the box. No airborne releases were reported for the Limerick site for 1985; therefore, none are plotted.

We should point out here, however, that the doses estimated in this study are extremely low compared to an average annual background dose of approximately $100 \mathrm{mrem}$. We have compared dose commitments calculated in this study with annual background. However, this comparison is not quite exact, since these dose commitments are those total-body doses received from the year's (1985) effluent release over 50 years of a person's lifetime. However, most of the dose commitment calculated here is delivered in the first year, so the comparison is reasonably valid. 


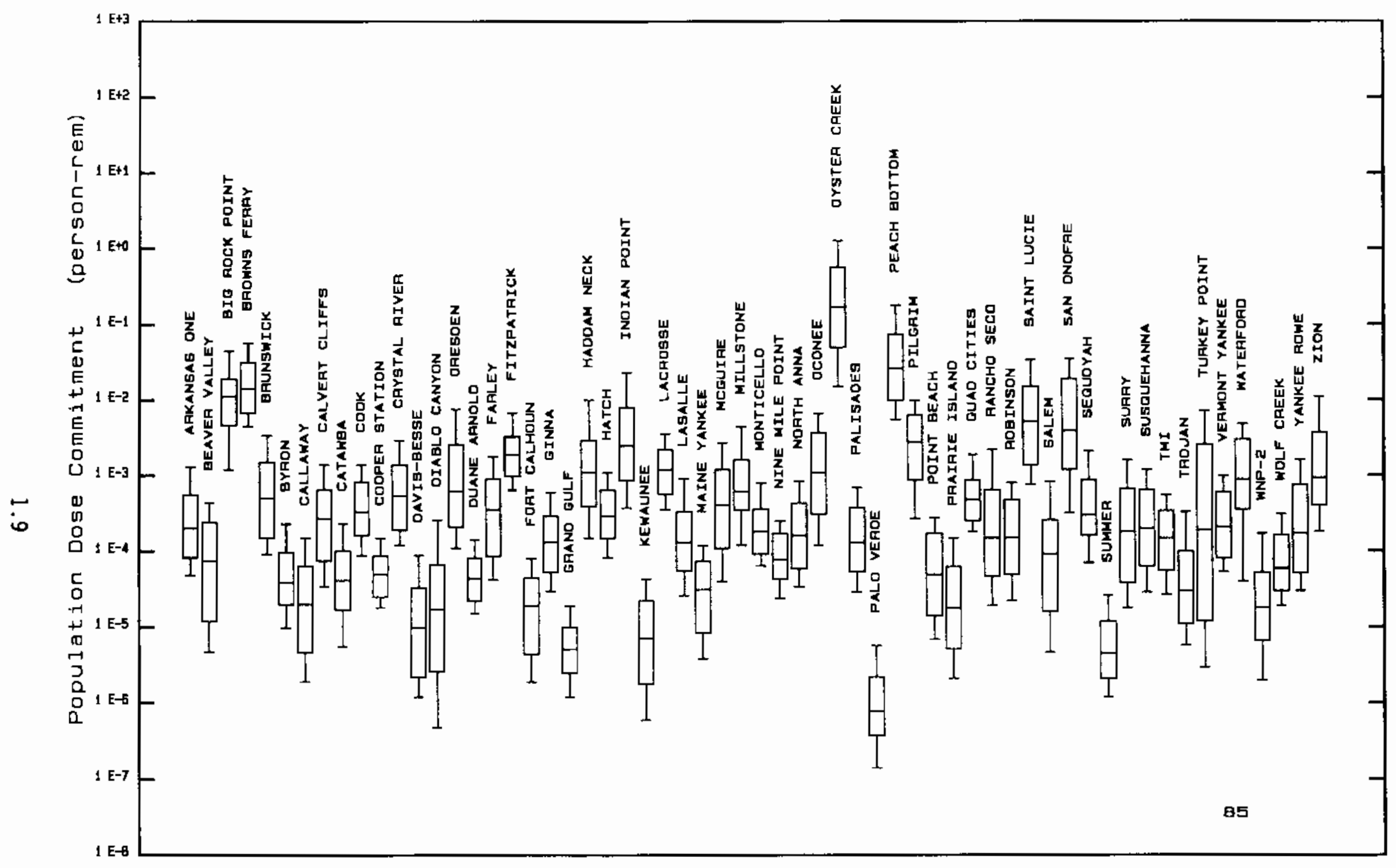

FIGURE 2. Airborne Population Dose Commitment for the Reactor Sites, 1985 
For comparison purposes, the doses in the site summary tables are given to two significant figures; however, the data and models used to calculate the doses limit their accuracy to at most one significant figure. 


\section{SITE COMPARISONS}

Compared to 1984 , the total dose from waterborne pathways was lower (91 vs. 160), and also, the total dose from airborne pathways was lower (110 vs. 120). Table 5 compares the total population dose commitments estimated for the past 11 years.

TABLE 5. Comparison of Annual Population Dose Commitments for the Past 11 Years (person-rem)

$\begin{array}{lllllllllll}1975 & 1976 & 1977 & 1978 & \underline{1979} & \underline{1980} & 1981 & 1982 & \underline{1983} & 1984 & 1985\end{array}$

$\begin{array}{lrrrrrrrrrrr}\text { Liquid } & 76 & 82 & 160 & 110 & 220 & 120 & 87 & 50 & 95 & 160 & 91 \\ \text { Air } & \underline{1300} & \underline{390} & \underline{540} & \underline{530} & \underline{1600} & -\frac{57}{16} & \underline{63} & \frac{87}{150} & \underline{76} & \underline{120} & \underline{110} \\ \quad \text { TOTAL } & 1300 & 470 & 700 & 640 & 1800 & 180 & 150 & 140 & 170 & 280 & 200\end{array}$

The reactor sites were compared as to the total population dose over the years of this study, 1975-1985. The sites were placed within six groupings depending on resulting population dose summed over each of the years through 1985:

$\begin{array}{llc}\text { I } & \text { Greater than } 100 \text { person-rem } \\ \text { II } & 26-100 & " \\ \text { III } & 11-25 & " \\ \text { IV } & 2.6-10 & " \\ \text { V } & 1-2.5 & " \\ \text { VI } & \text { Less than } 1 & "\end{array}$

Table 6 shows the sites within the groups along with the reactor manufacturer, year of commercial operation commencement, and the indicated population doses in person-rem. The manufacturer codes are as follows:
AC Allis Chalmers
B Babcox and Wilcox
CE Combustion Engineering
GE General Electric
W Westinghouse 


\section{TABLE 6. Total-Body Population Doses from Nuclear Power Plant Effluents During Norma? Operations, (a) 1975-1985}

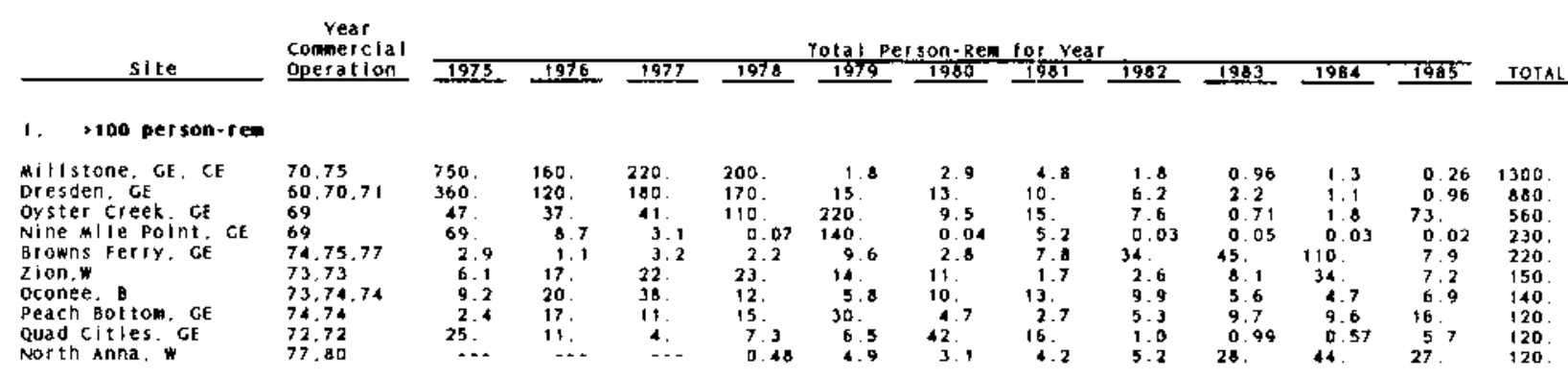

11.26 - 100 person-rea
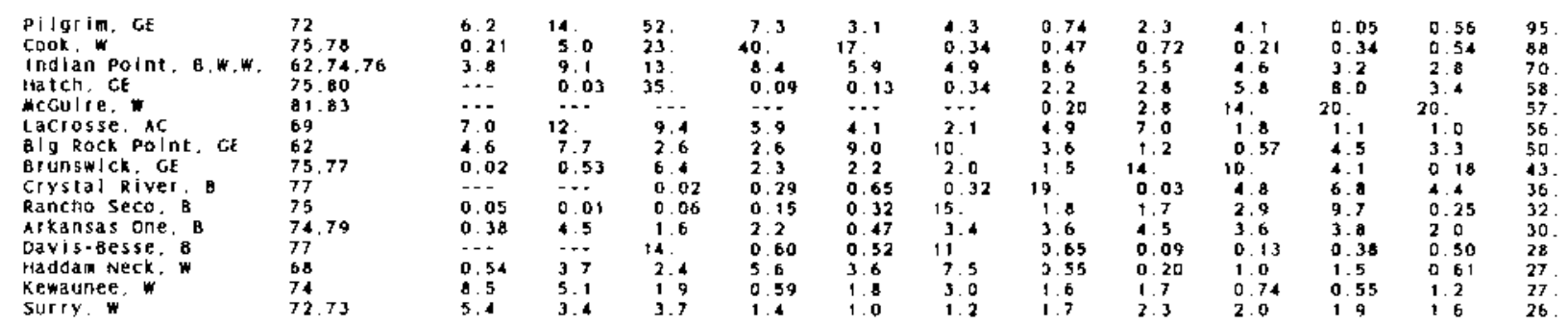

111. 11 - 25 person-rem
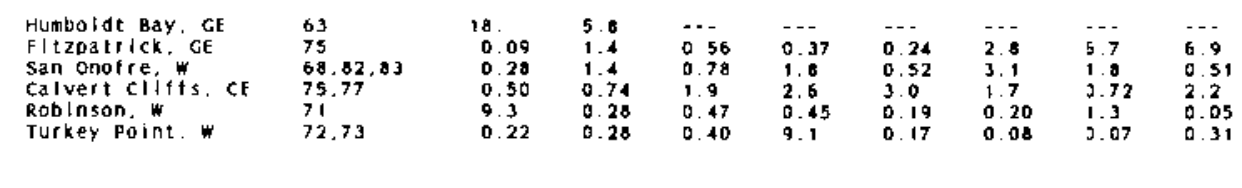

$0 .-$
2.8
0.71
0.92
0.44
0.26

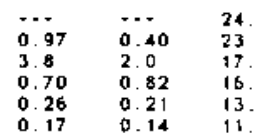

iv. 2,6 - 10 person-rea
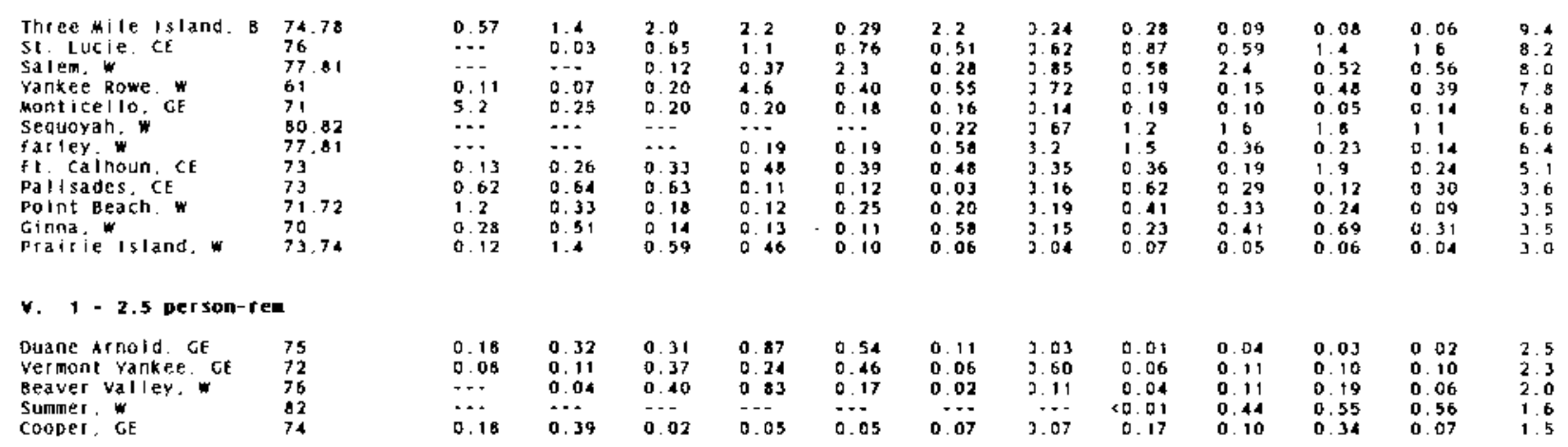

vi. 1 derson-rem

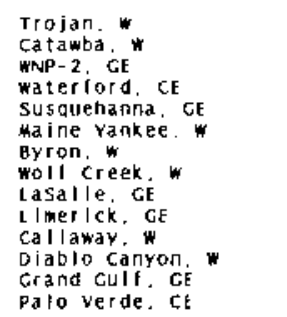

TOIAL

76
85
84
85
82
72
85
85
82
84
84
84
83
85

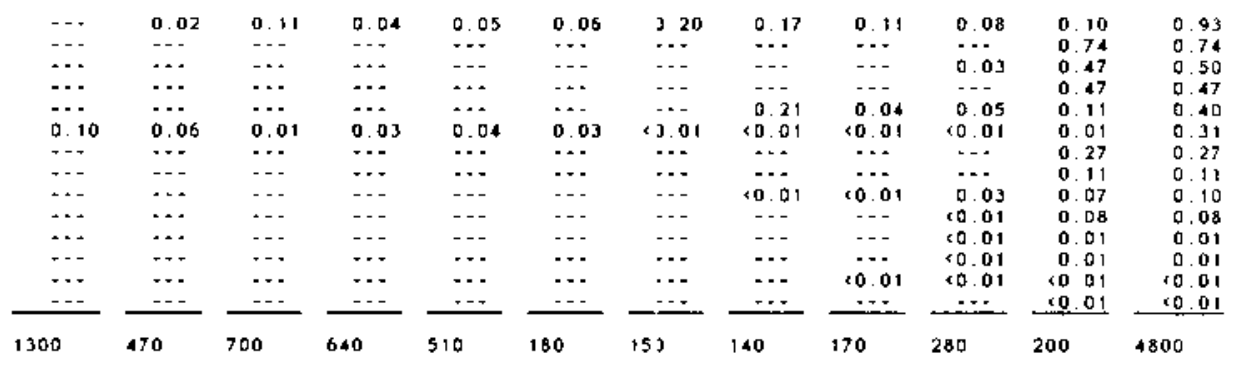

(a) Only the doses from the THA accident, 1979. are excluded. 


\section{SITE SUMMARIES}

1985

2.1 
Site: ARKANSAS ONE

POPE COUNTY, ARKANSAS

Location: $\quad$ N $35.3100^{\circ}$ W $93.2308^{\circ}$

POPULATION DATA

Total Population Within 2-to-80-km Region: 1.9E5

Major Metropolitan Centers Within Region:

\begin{tabular}{lccc}
\multicolumn{1}{c}{ Center } & Population & \multicolumn{2}{c}{ Location } \\
Russellville & 14,000 & $10 \mathrm{~km}$ & $\mathrm{E}$ \\
Conway & 20,000 & $76 \mathrm{~km}$ & ESE
\end{tabular}

SITE-SPECIFIC DATA - AIRBORNE PATHWAYS

Average Annual State Production

of Crops and Animal Products

In 80-km Radius Circle

Veg: $5.8 \mathrm{E} 6$ kilogram

Milk: 4.8E7 liter

Meat: $7.2 E 7$ kilogram

Regional Productivity Factor:

1

Animal Grazing Factor:

0.7

Meteorology Period of Record: 1 JAN 75 - 31 DEC 75 Recovery: $97 \%$

SITE-SPECIFIC DATA - WATERBORNE PATHWAYS via ARKANSAS RIVER

Average River Flow

at Site: $36,000 \mathrm{ft}^{3} / \mathrm{s}$

Drinking Water:

Exposed Population: None

Fish:

Edible Harvest: $1.4^{(a)} \mathrm{kg} / \mathrm{yr}$

Dilution Factor: 1

(a) Average individual consumption rates as given in the Final Environmental Statement (FES) (1973) were used in 1 ieu of catch data. The reference to environmental statements here and on succeeding pages may be found in Table 3. 


\begin{abstract}
POPULATION DOSE-COMMITMENT ESTIMATES AND
AVERAGE INDIVIDUAL DOSE-COMMITMENT HISTOGRAM FOR
\end{abstract}

ARKANSAS ONE 1 AND 2

Dose Commitments (person-rem) from Waterborne Pathways

Total Body GI-LLI $\underline{\text { Thyroid }}$ Bone Liver

$\begin{array}{llllll}\text { Infant } & 0.0 \mathrm{E}+00 & 0.0 \mathrm{E}+00 & 0.0 \mathrm{E}+00 & 0.0 \mathrm{E}+00 & 0.0 \mathrm{E}+00 \\ \text { Child } & 8.0 \mathrm{E}-02 & 2.7 \mathrm{E}-02 & 4.0 \mathrm{E}-02 & 4.2 \mathrm{E}-01 & 4.7 \mathrm{E}-01 \\ \text { Teen } & 1.5 \mathrm{E}-01 & 5.5 \mathrm{E}-02 & 2.9 \mathrm{E}-02 & 2.5 \mathrm{E}-01 & 3.9 \mathrm{E}-01 \\ \text { Aduit } & 1.7 \mathrm{E}+00 & 4.8 \mathrm{E}-01 & 1.9 \mathrm{E}-01 & 1.4 \mathrm{E}+00 & 2.3 \mathrm{E}+00 \\ \text { TOTAL } & 1.9 \mathrm{E}+00 & 5.6 \mathrm{E}-01 & 2.6 \mathrm{E}-01 & 2.1 \mathrm{E}+00 & 3.2 \mathrm{E}+00\end{array}$

Dose Commitments (person-rem) from Airborne Pathways

Total Body GI-LLI Ihyroid Bone Liver Lung

$\begin{array}{lllllll}\text { Infant } & 1.6 \mathrm{E}-03 & 1.6 \mathrm{E}-03 & 2.5 \mathrm{E}-03 & 1.5 \mathrm{E}-03 & 1.6 \mathrm{E}-03 & 1.7 \mathrm{E}-03 \\ \text { Child } & 1.7 \mathrm{E}-02 & 1.7 \mathrm{E}-02 & 2.3 \mathrm{E}-02 & 1.7 \mathrm{E}-02 & 1.7 \mathrm{E}-02 & 1.9 \mathrm{E}-02 \\ \text { Teen } & 1.3 \mathrm{E}-02 & 1.3 \mathrm{E}-02 & 1.5 \mathrm{E}-02 & 1.3 \mathrm{E}-02 & 1.3 \mathrm{E}-02 & 1.5 \mathrm{E}-02 \\ \text { Adult } & 7.7 \mathrm{E}-02 & 7.7 \mathrm{E}-02 & \mathbf{8 . 5 \mathrm { E } - 0 2} & 7.6 \mathrm{E}-02 & 7.7 \mathrm{E}-02 & \mathbf{8 . 6 \mathrm { E } - 0 2} \\ \text { TOTAL } & 1.1 \mathrm{E}-01 & 1.1 \mathrm{E}-01 & 1.3 \mathrm{E}-01 & 1.1 \mathrm{E}-01 & 1.1 \mathrm{E}-01 & 1.2 \mathrm{E}-01\end{array}$

Production/Consumption factors: (a)

Produce: 0.16 Milk: $1.9 \quad$ Meat: 4.7

Fraction of Population Receiving an Average Individual

Total-Body Dose Commitment from Airoorne Pathways

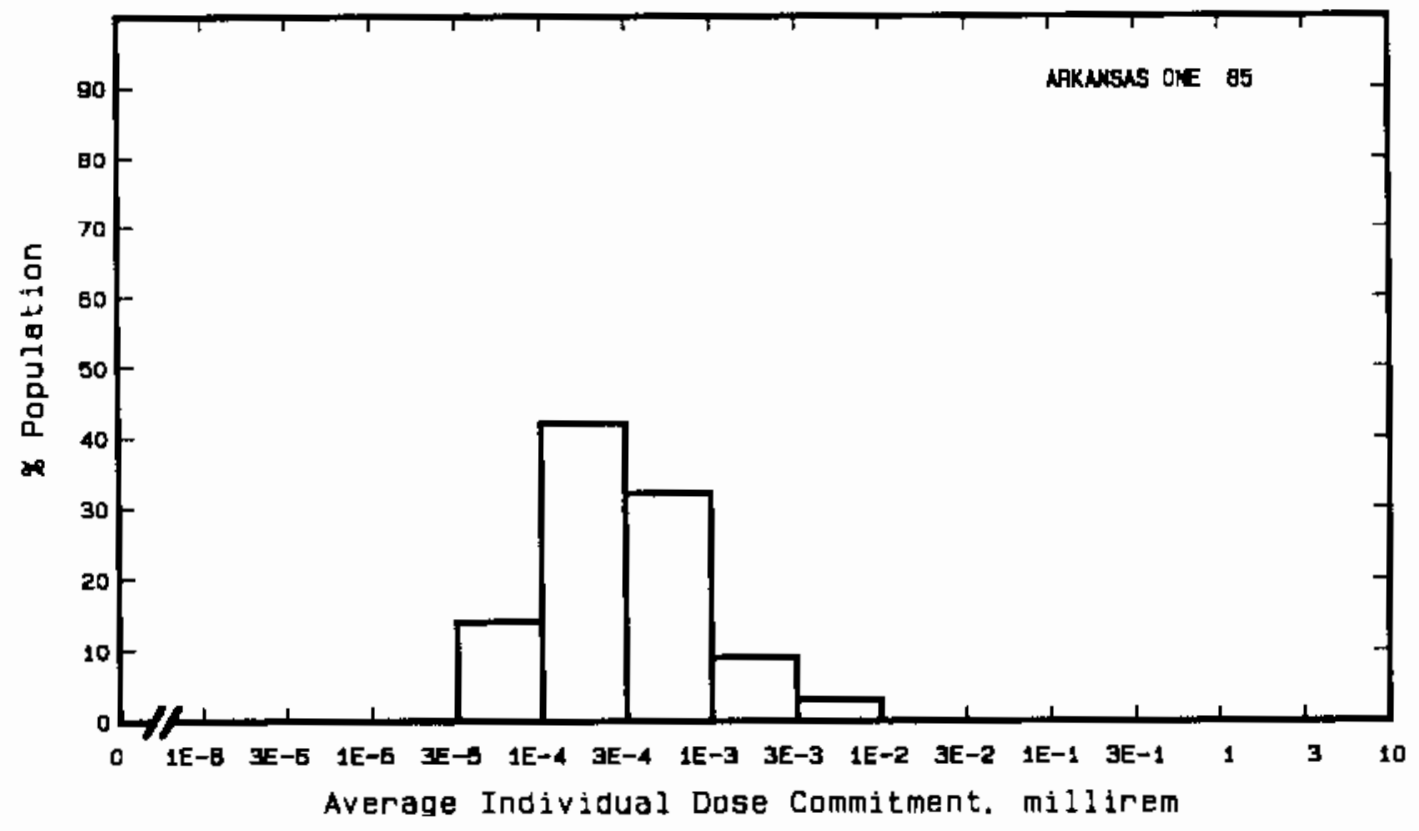

(a) See Appendix A, Page A-5, for explanation of this ratio. 
Site: BEAVER VALLEY

SHIPPINGPORT, PENNSYLVANIA

Location: $\quad$ N $40.6219^{\circ} \quad$ W $80.4339^{\circ}$

POPULATION DATA

Tota1 Population Within 2-to-80-km Region: 3.6E6

Major Metropolitan Centers Within Region:

Center Population Location

Pittsburgh SMSA

Youngstown-Warren SMSA

Stuebenville-Weirton SMSA

Wheeling SMSA

New Castle

$2,100,000$
510,000
160,000
190,000
34,000

$42 \mathrm{~km} \quad \mathrm{ESE}$

$56 \mathrm{~km} \quad \mathrm{NNW}$

$33 \mathrm{~km} \quad \mathrm{SSW}$

$66 \mathrm{~km} \quad \mathrm{SSW}$

$43 \mathrm{~km} \mathrm{~N}$

SITE-SPECIFIC DATA - AIRBORNE PATHWAYS

Average Annual State Production

of Crops and Animal Products

In $80-\mathbf{k m}$ Radius Circle

Regional Productivity Factor:

Animal Grazing Factor:

Meteorology Period of Record: 1 JAN 77 - 31 DEC 77 Recovery: $92 \%$
Veg: $5.3 \mathrm{E} 7 \mathrm{ki}$ logram

Milk: 5.3E8 liter

Meat: $5.4 \mathrm{E} 7 \mathrm{kilogram}$

1

0.5

SITE-SPECIFIC DATA - WATERBORNE PATHWAYS via OHIO RIVER

Average River $\mathrm{Fl}$ ow at Site: $30,000 \mathrm{ft}^{3} / \mathrm{s}$

Drinking Water:

Exposed Population: 6,200

Dilution Factor: $12(a)$

Fish:

Edible Harvest: $410 \mathrm{~kg} / \mathrm{yr}$ Dilution Factor: 1

(a) This factor accounts for the incomplete dilution of plant effluent by river at point of drinking water intake at Midland. 
POPULATION DOSE-COMMITMENT ESTIMATES AND

AVERAGE INDIVIDUAL DOSE-COMMITMENT HISTOGRAM FOR

\section{BEAYER VALLEY}

Dose Commitments (person-rem) from Waterborne Pathways

Total Body GI-LLI Ihyroid Bone Liver

$\begin{array}{llllll}\text { Infant } & 3.3 \mathrm{E}-04 & 3.3 \mathrm{E}-04 & 3.1 \mathrm{E}-04 & 4.2 \mathrm{E}-05 & 3.7 \mathrm{E}-04 \\ \text { Child } & 3.7 \mathrm{E}-03 & 3.8 \mathrm{E}-03 & 3.5 \mathrm{E}-03 & 4.6 \mathrm{E}-04 & 4.0 \mathrm{E}-03 \\ \text { Teen } & 1.4 \mathrm{E}-03 & 1.6 \mathrm{E}-03 & 1.3 \mathrm{E}-03 & 1.2 \mathrm{E}-04 & 1.5 \mathrm{E}-03 \\ \text { Adult } & 1.2 \mathrm{E}-02 & 1.4 \mathrm{E}-02 & 1.1 \mathrm{E}-02 & 7.3 \mathrm{E}-04 & 1.3 \mathrm{E}-02 \\ \text { TOTAL } & 1.8 \mathrm{E}-02 & 1.9 \mathrm{E}-02 & 1.7 \mathrm{E}-02 & 1.4 \mathrm{E}-03 & 1.9 \mathrm{E}-02\end{array}$

Dose Commitments (person-rem) from Airborne Pathways

Total Body GI-LLI Thyroid Bone Liver Lung

$\begin{array}{lllllll}\text { Infant } & 6.0 \mathrm{E}-04 & 5.1 \mathrm{E}-04 & 1.4 \mathrm{E}-03 & 5.8 \mathrm{E}-04 & 5.2 \mathrm{E}-04 & 5.9 \mathrm{E}-04 \\ \text { Child } & 8.2 \mathrm{E}-03 & 6.1 \mathrm{E}-03 & 1.1 \mathrm{E}-02 & 1.4 \mathrm{E}-02 & 6.1 \mathrm{E}-03 & 7.3 \mathrm{E}-03 \\ \text { Teen } & 4.9 \mathrm{E}-03 & 3.9 \mathrm{E}-03 & 5.9 \mathrm{E}-03 & 8.6 \mathrm{E}-03 & 3.8 \mathrm{E}-03 & 4.9 \mathrm{E}-03 \\ \text { Adult } & 2.4 \mathrm{E}-02 & 2.0 \mathrm{E}-02 & 2.7 \mathrm{E}-02 & 4.3 \mathrm{E}-02 & 2.0 \mathrm{E}-02 & 2.4 \mathrm{E}-02 \\ \text { TOTAL } & 3.8 \mathrm{E}-02 & 3.1 \mathrm{E}-02 & 4.5 \mathrm{E}+02 & 6.6 \mathrm{E}-02 & 3.0 \mathrm{E}-02 & 3.7 \mathrm{E}-02\end{array}$

Production/Consumption factors:
Produce: 0.076
Milk: 1.1
Meat: 0.19

Fraction of Population Receiving an Average Individual

Total-Body Dose Commitment from Airborne Pathways

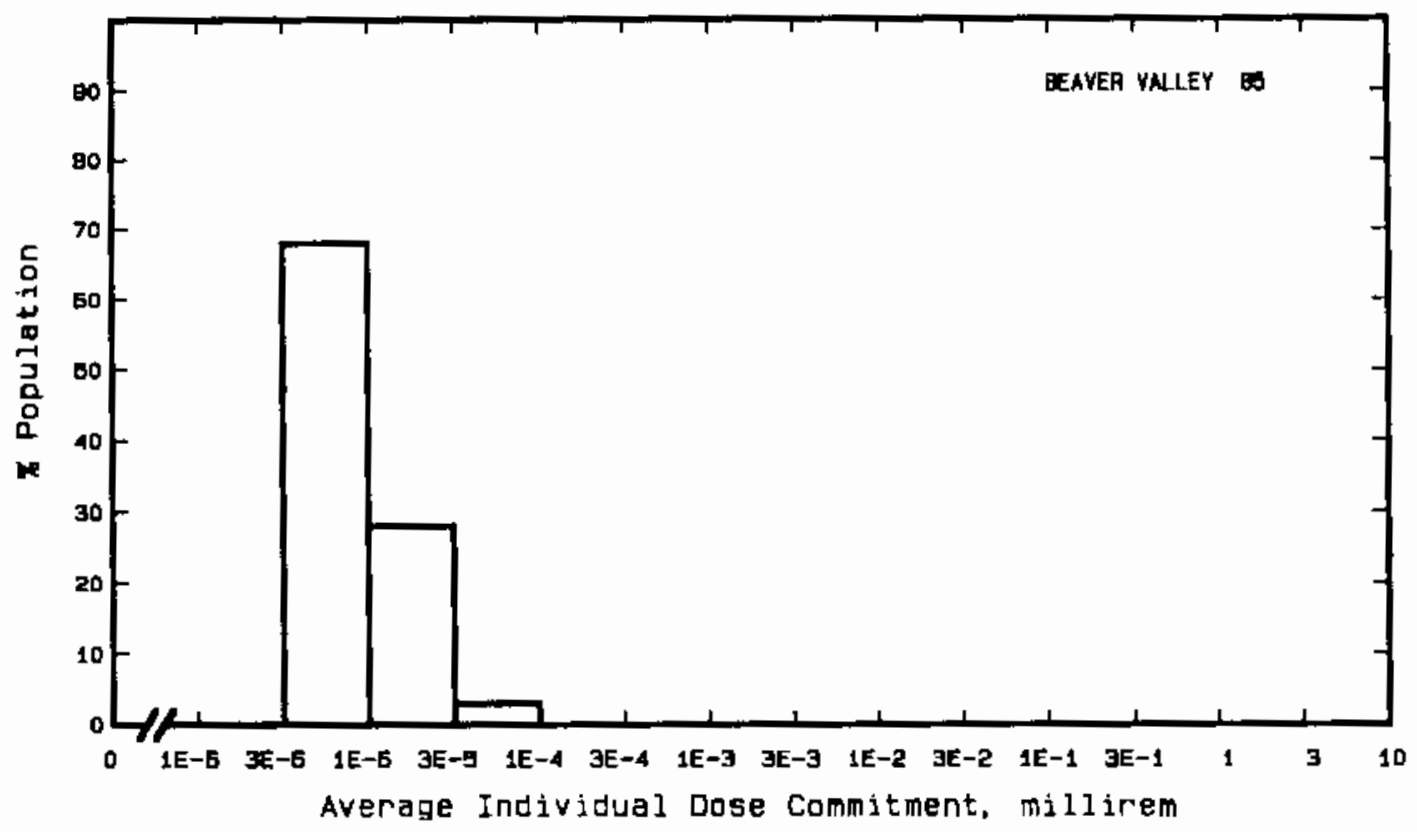


Site: BIG ROCK POINT

CHARLEVOIX COUNTY, MICHIGAN

Location: $\quad$ N $45.3592^{\circ} \quad$ W $85.1947^{\circ}$

POPULATION DATA

Total Population Within 2-to-80-km Region: 1.6E5

Major Metropolitan Centers Within Region:

Center

Traverse City

Petoskey

Cheboygan
Population

16,000

6,100

5,100
Location

$75 \mathrm{~km} \quad \mathrm{SSW}$

$18 \mathrm{~km} \quad \mathrm{E}$

$65 \mathrm{~km} \quad \mathrm{ENE}$

SITE-SPECIFIC DATA - AIRBORNE PATHWAYS

Average Annual State Production

of Crops and Animal Products

In $80-\mathrm{km}$ Radius Circle

Regional Productivity Factor:

Animal Grazing Factor:
Veg: $6.9 E 7 \mathrm{ki}$ logram

Milk: 2.9E8 liter

Meat: 4.5E7 kilogram

0.5

0.5

Meteorology Period of Record: 9 FEB 61 - 8 FEE 63 Recovery: 85\%

SITE-SPECIFIC DATA - WATERBORNE PATHWAYS via LAKE MICHIGAN

Average Dilution Flow

from Plant: $110 \mathrm{ft}^{3} / \mathrm{s}$

Orinking Water:

Exposed Population: 30,000 (a)

Dilution Factor: $4.9 \mathrm{E}-5(\mathrm{~b})$

Fish:

Edible Harvest: (c) $\mathrm{kg} / \mathrm{yr}$

Dilution Factor: 0.01

(a) Population exposed to contaminated drinking water derived from information obtained from J. Hennigan, Division of Radiation Health, Bureau of Environment and Health, Michigan Department of Public Health.

(b) Drinking water dilution factor estimated by averaging dilution factor derived from Figure 6B-5, Vol. I of WASH-1258 (1973) suitably weighted for population.

(c) Generic consumption rate used (Table A-1). 


\section{POPULATION DOSE-COMMITMENT ESTIMATES AND AVERAGE INDDIVIDUAL DOSE-COMMITMENT HISTOGRAM FOR}

BIG ROCK POINT

Dose Commitments (person-rem) from Waterborne Pathways

Total Body GI-LLI $\underline{\text { Thyroid }}$ Bone

Infant

$2.6 \mathrm{E}-07$

$7.3 \mathrm{E}-08$

$1.2 \mathrm{E}-05$

$1.2 \mathrm{E}-06$

$1.0 \mathrm{E}-06$

Child

$2.8 \mathrm{E}-02$

$1.9 \mathrm{E}-03$

$6.9 \mathrm{E}-03$

1. $5 \mathrm{E}-01$

1. $6 \mathrm{E}-01$

Teen

5. 3E-02

$4.0 \mathrm{E}-03$

$5.0 \mathrm{E}-03$

8.8E-02

$1.4 \mathrm{E}-01$

Adult

$5.8 \mathrm{E}-01$

3. $5 \mathrm{E}-02$

3. $3 \mathrm{E}-02$

5. $1 \mathrm{E}-01$

$8.1 \mathrm{E}-01$

TOTAL

$6.6 \mathrm{E}-01$

4. OE-02

4.5E-02

7. $4 \mathrm{E}-01$

1. $\mathrm{E}+00$

Dose Commitments (person-rem) from Airborne Pathways

Total Body GI-LLI Thyroid Bone Liver

$\begin{array}{lllllll}\text { Infant } & 3.8 \mathrm{E}-02 & 3.8 \mathrm{E}-02 & 4.1 \mathrm{E}-02 & 3.8 \mathrm{E}-02 & 3.8 \mathrm{E}-02 & 3.9 \mathrm{E}-02 \\ \text { Child } & 4.2 \mathrm{E}-01 & 4.2 \mathrm{E}-01 & 4.6 \mathrm{E}-01 & 4.2 \mathrm{E}-01 & 4.2 \mathrm{E}-01 & 4.3 \mathrm{E}-01 \\ \text { Teen } & 3.1 \mathrm{E}-01 & 3.1 \mathrm{E}-01 & 3.2 \mathrm{E}-01 & 3.1 \mathrm{E}-01 & 3.1 \mathrm{E}-01 & 3.2 \mathrm{E}-01 \\ \text { Aduit } & 1.9 \mathrm{E}+00 & 1.9 \mathrm{E}+00 & 1.9 \mathrm{E}+00 & 1.9 \mathrm{E}+00 & 1.9 \mathrm{E}+00 & 1.9 \mathrm{E}+00 \\ \text { TOTAL } & 2.6 \mathrm{E}+00 & 2.6 \mathrm{E}+00 & 2.7 \mathrm{E}+00 & 2.6 \mathrm{E}+00 & 2.6 \mathrm{E}+00 & 2.7 \mathrm{E}+00\end{array}$

Production/Consumption factors:

Produce: 1.1

Milk: 6.6

Meat: 1.7

Fraction of Population Receiving an Average Individual

Tota1-Gody Dose Commitment from Airborne Pathways

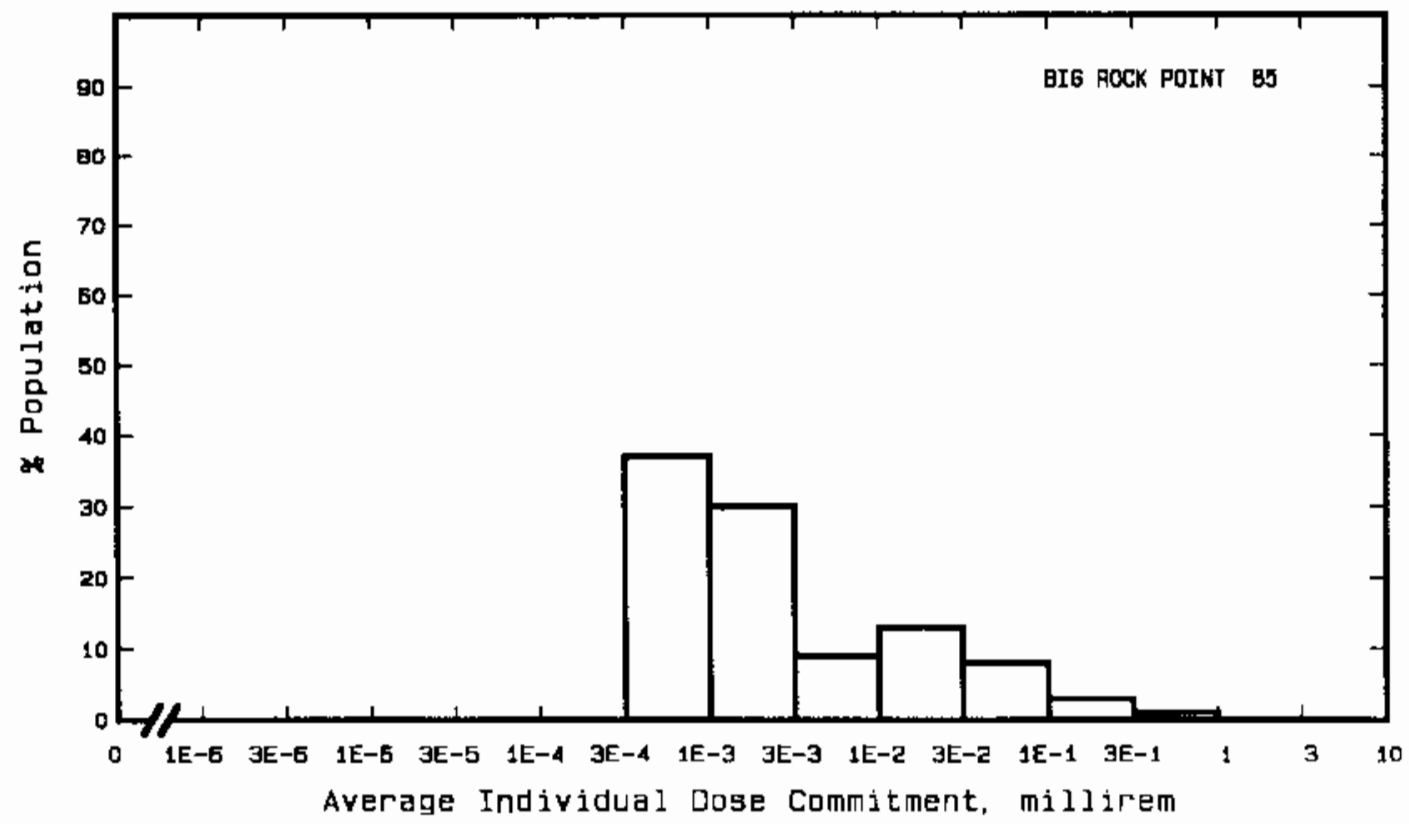


Site: BROWNS FERRY

Location: N $34.7042^{\circ}$
DECATUR, ALABAMA

W $87.1186^{\circ}$

POPULATION DATA

Total Population Within 2-to-80-km Region: $7.4 \mathrm{E5}$

Major Metropolitan Centers Within Region:

\begin{tabular}{lrlll}
\multicolumn{1}{c}{ Center } & Population & \multicolumn{2}{c}{ Location } \\
\cline { 2 - 3 } & & & \\
Huntsville SMSA & 310,000 & $59 \mathrm{~km}$ & $\mathrm{E}$ \\
Florence SMSA & 140,000 & $16 \mathrm{~km}$ & SEW \\
Decatur & 42,000 & $17 \mathrm{~km}$ & $\mathrm{NE}$ \\
Athens & 15,000 & $64 \mathrm{~km}$ & SSE \\
Cullman & 13,000 & &
\end{tabular}

SITE-SPECIFIC DATA - AIRBORNE PATHWAYS

Average Annual State Production

of Crops and Animal Products

In 80-km Radius Circle

Regional Productivity Factor:

Animal Grazing Factor:
Veg: 1.7E7 kilogram

Milk: 5.7E7 liter

Heat: $8.6 \mathrm{E} 7 \mathrm{kjlogram}$

1

0.7

Meteorology Period of Record: 1 JAN 74 - 31 [DEC 75 Recovery: $94 \%$

SITE-SPECIFIC DATA - WATERBORNE PATHWAYS via TENNESSEE RIVER at WHEELER

Drinking Water:

Fish:
Average River Flow

at Site: $45,0010 \mathrm{ft}^{3} / \mathrm{s}$

Exposed Population: 26,000

Dilution Factor: 1

Edible Harvest: $1.6 \mathrm{E} 6 \mathrm{~kg} / \mathrm{yr}$

Dilution Factor: 1 


\section{POPULATION DOSE-COMMITMENT ESTIMATES AND \\ AVERAGE INDIVIDUAL DOSE-COMMITMENT HISTOGRAM FOR \\ BROWNS FERRY 1,2 AND 3}

Dose Commitments (person-rem) from Waterborne Pathways

Total Body $\underline{\text { GI-LLI }}$ Thyroid $\underline{\text { Bone }}$ Liver

$\begin{array}{llllll}\text { Infant } & 7.2 \mathrm{E}-05 & 4.4 \mathrm{E}-05 & 3.2 \mathrm{E}-04 & 3.6 \mathrm{E}-04 & 5.1 \mathrm{E}-04 \\ \text { Child } & 1.6 \mathrm{E}-01 & 1.4 \mathrm{E}-02 & 3.6 \mathrm{E}-03 & 6.5 \mathrm{E}-01 & 8.0 \mathrm{E}-01 \\ \text { Teen } & 2.8 \mathrm{E}-01 & 2.9 \mathrm{E}-02 & 1.7 \mathrm{E}-03 & 3.9 \mathrm{E}-01 & 6.8 \mathrm{E}-01 \\ \text { Adult } & 2.9 \mathrm{E}+00 & 2.6 \mathrm{E}-01 & 1.1 \mathrm{E}-02 & 2.3 \mathrm{E}+00 & 4.1 \mathrm{E}+00 \\ \text { TOTAL } & 3.4 \mathrm{E}+00 & 3.0 \mathrm{E}-01 & 1.7 \mathrm{E}-02 & 3.3 \mathrm{E}+00 & 5.6 \mathrm{E}+00\end{array}$

Dose Commitments (person-rem) from Airborne Pathways

Total Body GI-LLI Thyroid Bone Liver Lung

$\begin{array}{lllllll}\text { Infant } & 6.5 \mathrm{E}-02 & 6.5 \mathrm{E}-02 & 7.2 \mathrm{E}-02 & 6.5 \mathrm{E}-02 & 6.5 \mathrm{E}-02 & 6.7 \mathrm{E}-02 \\ \text { Child } & 7.2 \mathrm{E}-01 & 7.2 \mathrm{E}-01 & 7.7 \mathrm{E}-01 & 7.2 \mathrm{E}-01 & 7.2 \mathrm{E}-01 & 7.5 \mathrm{E}-0 \mathrm{1} \\ \text { Teen } & 5.3 \mathrm{E}-01 & 5.3 \mathrm{E}-01 & 5.5 \mathrm{E}-01 & 5.3 \mathrm{E}-01 & 5.3 \mathrm{E}-01 & 5.6 \mathrm{E}-01 \\ \text { Adult } & 3.2 \mathrm{E}+00 & 3.2 \mathrm{E}+00 & 3.3 \mathrm{E}+00 & 3.2 \mathrm{E}+00 & 3.2 \mathrm{E}+00 & 3.3 \mathrm{E}+00 \\ \text { TOTAL } & 4.5 \mathrm{E}+00 & 4.5 \mathrm{E}+00 & 4.7 \mathrm{E}+00 & 4.5 \mathrm{E}+00 & 4.5 \mathrm{E}+00 & 4.7 \mathrm{E}+00\end{array}$

Production/Consumption factors:

Produce: 0.12 Milk: $0.59 \quad$ Meat: 1.5

Fraction of Population Receiving an Average Individual

Total-Body Dose Commitment from Airborne Pathways

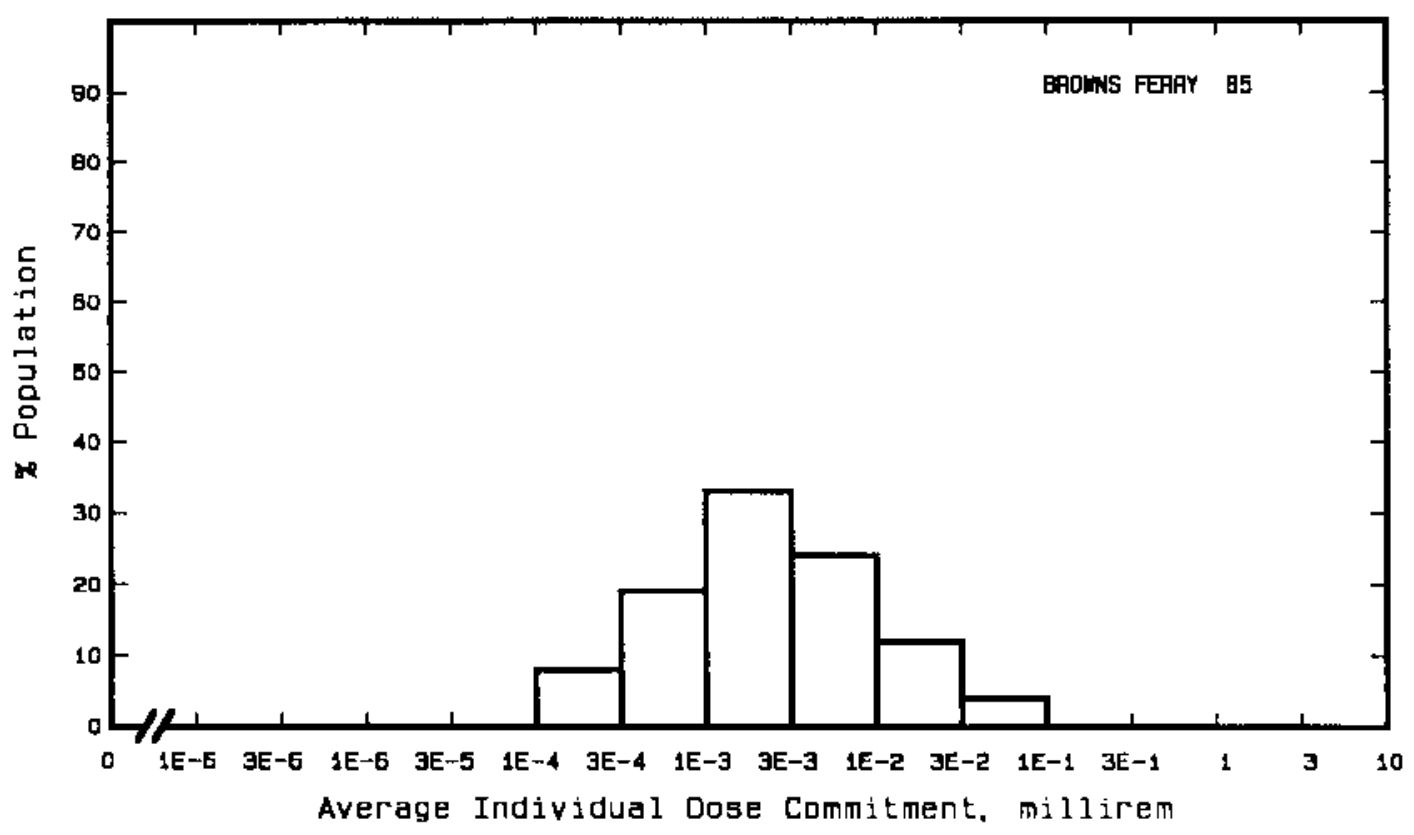


Site: BRUNSWICK

Location: $\quad N 33.9583^{\circ} \quad$ W $78.0106^{\circ}$

POPULATION DATA

Total Population Within 2-to-80-km Region: 2.3E5

Major Metropolitan Centers Within Region:

\begin{tabular}{lrrr}
\multicolumn{1}{c}{ Center } & Population & \multicolumn{2}{c}{ Location } \\
Wilmington SMSA & 140,000 & $32 \mathrm{~km}$ & NNE \\
Whiteville & 5,600 & $75 \mathrm{~km}$ & WNW \\
N. Myrtle Beach & 4,000 & $65 \mathrm{~km}$ & WSW
\end{tabular}

SITE-SPECIFIC DATA - AIRBORNE PATHWAYS

Average Annual State Production

of Crops and Animal Products

In $80-\mathrm{km}$ Radius Circle

Regional Productivity Factor:

Animal Grazing Factor:
Veg: $2.6 E 7$ kilogram

Mi7k: $1.0 \mathrm{E} 8$ liter

Meat: $5.8 E 7$ kilogram

0.3

0.7

Meteorology Period of Record: 1 JAN 75 - 31 DEC 75 Recovery: 93\%

\section{SITE-SPECIFIC DATA - WATERBORNE PATHWAYS via ATLANTIC OCEAN}

Average Dilution Flow

from PIant: $88 \mathrm{ft}^{3} / \mathrm{s}$

Fish:

Edible Harvest: $2.1 \mathrm{E5}(\mathrm{a}) \mathrm{kg} / \mathrm{yr}$

Dilution Factor: 0.001

Invertebrates:

Edible Harvest: $1.1 \mathrm{E5}$ (a) $\mathrm{kg} / \mathrm{yr}$

Dilution Factor: 0.002

(a) Fish and invertebrate harvests together total harvest given in FES (1973). 


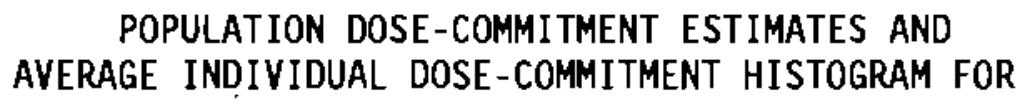

BRUNSWICK 1 AND 2

Dose Commitments (person-rem) from Waterborne Pathways

Total Body GI-LLI Ihyroid Bone

$\begin{array}{llllll}\text { Infant } & 0.0 \mathrm{E}+00 & 0.0 \mathrm{E}+00 & 0.0 \mathrm{E}+00 & 0.0 \mathrm{E}+00 & 0.0 \mathrm{E}+00 \\ \text { Child } & 2.6 \mathrm{E}-04 & 3.9 \mathrm{E}-04 & 6.7 \mathrm{E}-06 & 5.3 \mathrm{E}-04 & 3.9 \mathrm{E}-04 \\ \text { Teen } & 1.7 \mathrm{E}-04 & 8.1 \mathrm{E}-04 & 4.8 \mathrm{E}-06 & 3.0 \mathrm{E}-04 & 3.0 \mathrm{E}-04 \\ \text { Adult } & 1.1 \mathrm{E}-03 & 7.1 \mathrm{E}-03 & 3.2 \mathrm{E}-05 & 1.7 \mathrm{E}-03 & 1.8 \mathrm{E}-03 \\ \text { TOTAL } & 1.5 \mathrm{E}-03 & 8.3 \mathrm{E}-03 & 4.4 \mathrm{E}-05 & 2.6 \mathrm{E}-03 & 2.5 \mathrm{E}-03\end{array}$

Dose Commitments (person-rem) from Airborne Pathways

Total Body GI-LLI Ihyroid Bone Liver Lung

$\begin{array}{lllllll}\text { Infant } & 2.6 \mathrm{E}-03 & 2.6 \mathrm{E}-03 & 8.5 \mathrm{E}-03 & 2.6 \mathrm{E}-03 & 2.6 \mathrm{E}-03 & 2.7 \mathrm{E}-03 \\ \text { Child } & 2.9 \mathrm{E}-02 & 2.9 \mathrm{E}-02 & 6.5 \mathrm{E}-02 & 2.9 \mathrm{E}-02 & 2.9 \mathrm{E}-02 & 3.1 \mathrm{E}-02 \\ \text { Teen } & 2.1 \mathrm{E}-02 & 2.1 \mathrm{E}-02 & 3.6 \mathrm{E}-02 & 2.1 \mathrm{E}-02 & 2.1 \mathrm{E}-02 & 2.3 \mathrm{E}-02 \\ \text { Adult } & 1.3 \mathrm{E}-01 & 1.3 \mathrm{E}-01 & 1.8 \mathrm{E}-01 & 1.3 \mathrm{E}-01 & 1.3 \mathrm{E}-01 & 1.3 \mathrm{E}-01 \\ \text { TDTAL } & 1.8 \mathrm{E}-01 & 1.8 \mathrm{E}-01 & 2.9 \mathrm{E}-01 & 1.8 \mathrm{E}-01 & 1.8 \mathrm{E}-01 & 1.9 \mathrm{E}-0 \mathrm{I}\end{array}$

Production/Consumption factors:

Produce: 0.17 Milk: $1.0 \quad$ Meat: 0.94

Fraction of Population Recelving an Average Individual Total-Body Dose Commitment from Airborne Pathways

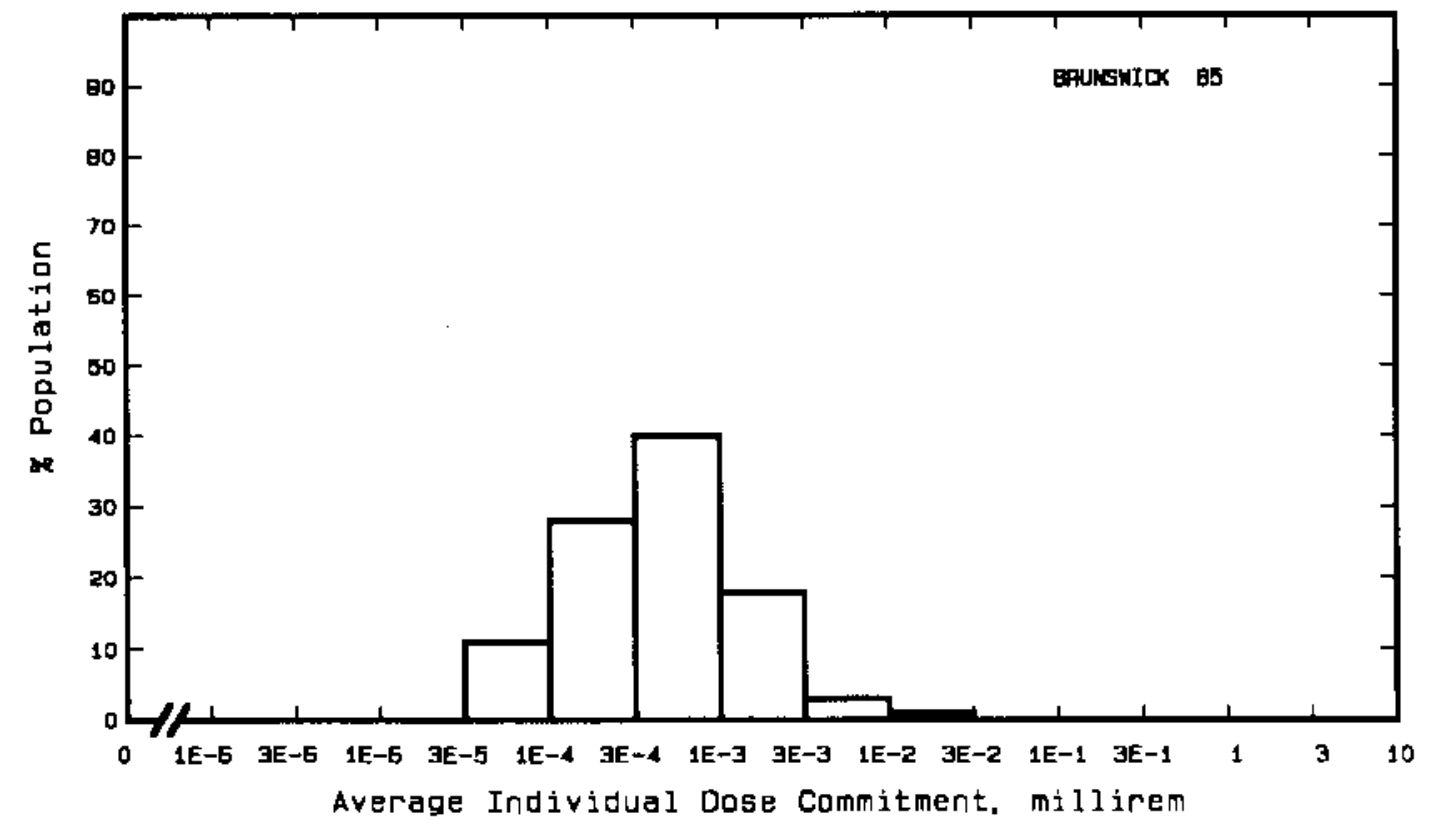


Site: BYRON

BYRON, ILLINOIS

Location: $\quad N \quad 42.1300^{\circ} \quad$ W $89.2550^{\circ}$

POPULATION DATA

Total Population Within 2-to-B0-km Region: 9.3E5

Major Metropolitan Centers Within Region:

\begin{tabular}{lrrrl}
\multicolumn{1}{c}{ Center } & Population & \multicolumn{2}{c}{ Location } \\
\cline { 4 - 6 } Rockford SMSA & 280,000 & & \\
Freeport & 26,000 & $27 \mathrm{~km}$ & $\mathrm{NE}$ \\
Belvedere & 42,000 & $35 \mathrm{~km}$ & NNW \\
Janesville & 61,000 & $40 \mathrm{~km}$ & $\mathrm{NE}$ \\
Beloit & 35,000 & $67 \mathrm{~km}$ & $\mathrm{~N}$ \\
De Kalb & 33,000 & $48 \mathrm{~km}$ & NNE \\
Elgin & 64,000 & $48 \mathrm{~km}$ & ESE \\
& & $80 \mathrm{~km}$ & E
\end{tabular}

\section{SITE-SPECIFIC DATA - AIRBORNE PATHWAYS}

Average Annual State Production

of Crops and Animal Products

In $80-\mathrm{km}$ Radius Circle

Regional Productivity Factor:

Animal Grazing Factor:
Veg: $1.1 \mathrm{E} 8 \mathrm{kilogram}$

Milk: 1.8E8 liter

Meat: $1.9 E 8$ kilogram

0.9

0.5

Meteorology Period of Record: 1 JAN 74 - 31 DEC 74 Recovery: 95\%

SITE-SPECIFIC DATA - WATERBORNE PATHWAYS via ROCK RIVER

Average River Flow

at Site: $4,600 \mathrm{ft}^{3} / \mathrm{s}$

Drinking Water:

Exposed Population: None

Dilution Factor:

Fish:

Edible Harvest: $2.1 \mathrm{E4} \mathrm{kg} / \mathrm{yr}$

Dilution Factor: 1 


\section{POPULATION DOSE-COMMITMENT ESTIMATES AND \\ AVERAGE INDIVIDUAL DOSE-COMMITMENT HISTOGRAM FOR}

BYRON

Dose Commitments (person-rem) from Waterborne Pathways

Total Body GI-LLI $\underline{\text { Thyroid }}$ Bone Liver

$\begin{array}{llllll}\text { Infant } & 0.0 \mathrm{E}+00 & 0.0 \mathrm{E}+00 & 0.0 \mathrm{E}+00 & 0.0 \mathrm{E}+00 & 0.0 \mathrm{E}+00 \\ \text { Child } & 1.2 \mathrm{E}-02 & 1.1 \mathrm{E}-02 & 2.8 \mathrm{E}-03 & 5.5 \mathrm{E}-02 & 6.1 \mathrm{E}-02 \\ \text { Teen } & 2.0 \mathrm{E}-02 & 2.4 \mathrm{E}-02 & 2.0 \mathrm{E}-03 & 3.3 \mathrm{E}+00 & 5.1 \mathrm{E}-02 \\ \text { Adult } & 2.1 \mathrm{E}-01 & 2.1 \mathrm{E}-01 & 1.3 \mathrm{E}-02 & 1.9 \mathrm{E}-01 & 3.0 \mathrm{E}-01 \\ \text { TOTAL } & 2.5 \mathrm{E}-01 & 2.4 \mathrm{E}-01 & 1.8 \mathrm{E}-02 & 2.8 \mathrm{E}-01 & 4.2 \mathrm{E}-01\end{array}$

Dose Commitments (person-rem) from Airborne Pathways

Total Body GI-LLI Ihyroid Bone Liver Lung

$\begin{array}{lllllll}\text { Infant } & 3.1 \mathrm{E}-04 & 3.1 \mathrm{E}-04 & 1.2 \mathrm{E}-03 & 3.1 \mathrm{E}-04 & 3.1 \mathrm{E}-04 & 3.3 \mathrm{E}-04 \\ \text { Child } & 3.5 \mathrm{E}-03 & 3.4 \mathrm{E}-03 & 1.1 \mathrm{E}-02 & 3.4 \mathrm{E}-03 & 3.5 \mathrm{E}-03 & 3.7 \mathrm{E}-03 \\ \text { Teen } & 2.5 \mathrm{E}-03 & 2.5 \mathrm{E}-03 & 5.4 \mathrm{E}-02 & 2.5 \mathrm{E}-03 & 2.5 \mathrm{E}-03 & 2.8 \mathrm{E}-03 \\ \text { Adult } & 1.5 \mathrm{E}-02 & 1.5 \mathrm{E}-02 & 2.6 \mathrm{E}-02 & 1.5 \mathrm{E}-02 & 1.5 \mathrm{E}-02 & 1.6 \mathrm{E}-02 \\ \text { TOTAL } & 2.1 \mathrm{E}-02 & 2.1 \mathrm{E}-02 & 4.3 \mathrm{E}-02 & 2.1 \mathrm{E}-02 & 2.2 \mathrm{E}-02 & 2.3 \mathrm{E}-02\end{array}$

Production/Consumption factors:
Produce: 0.56
Milk: 1.3
Meat: 2.3

Fraction of Population Receiving an Average Individual

Total-Body Dose Commitment from Airborne Pathways

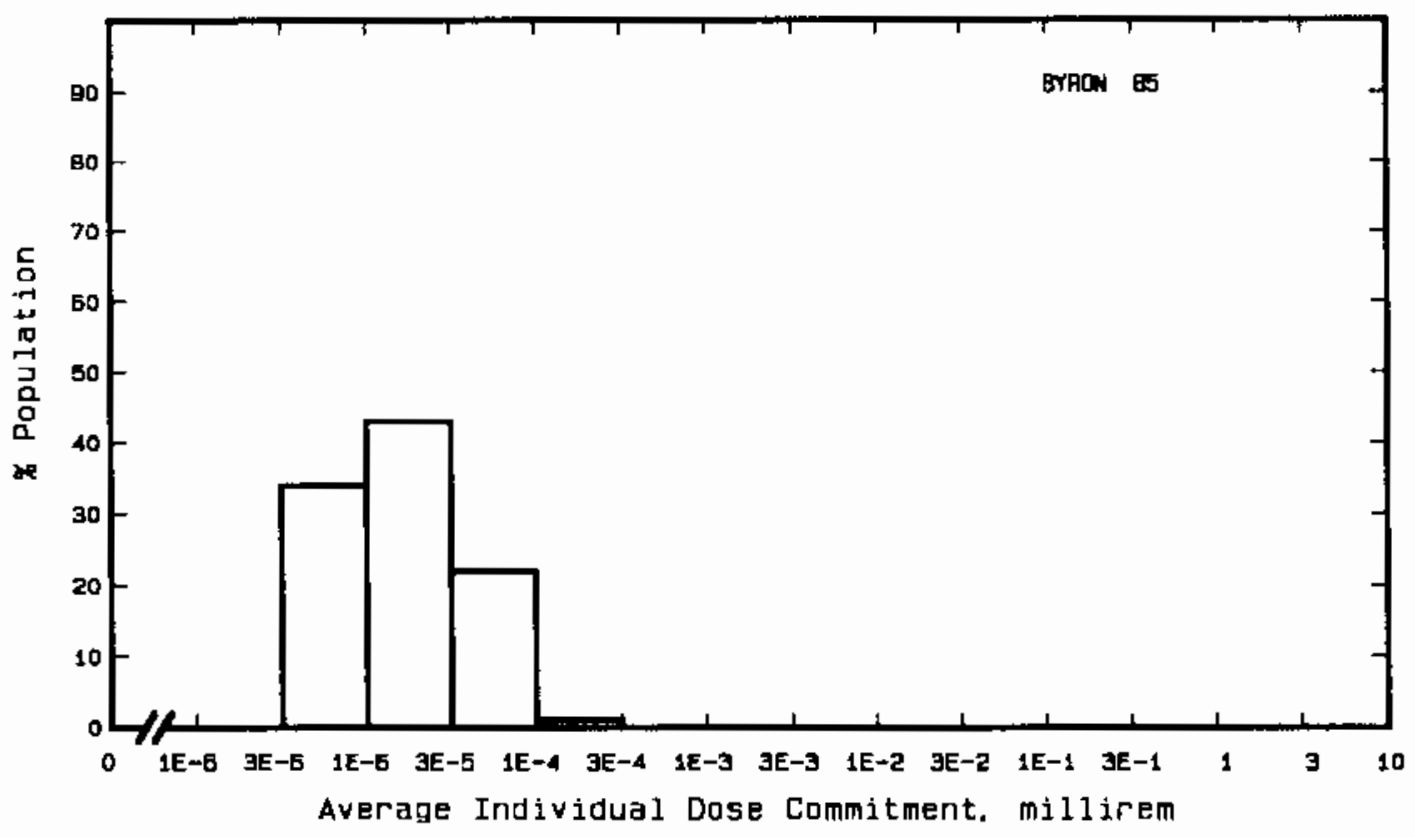


Site: CALLAWAY

FULTON, MISSOURI

Location: $\quad$ N $38.7618^{\circ} \quad$ W $91.7979^{\circ}$

POPULATION DATA

Total Population Within 2-to-80-km Region: 3.7 E5

Major Metropolitan Centers Within Region:

Center

Columbia

Jefferson City

Mexico

Washington

Fulton
Population

59,000

33,000

12,000

8,500

13,000
Location

$48 \mathrm{~km}$ WNW

$40 \mathrm{~km} \quad W S W$

$45 \mathrm{~km} \quad \mathrm{NNW}$

$69 \mathrm{~km} \quad \mathrm{ESE}$

$19 \mathrm{~km} \quad \mathrm{NW}$

SITE-SPECIFIC DATA - AIRBORNE PATHWAYS

Average Annual State Production

of Crops and Animal Products

In $80-\mathrm{km}$ Radius Circle

Regional Productivity Factor:

Animal Grazing Factor:
Veg: $3.3 \mathrm{E} 7 \mathrm{kilogram}$

Milk: $1.5 \mathrm{E} 8$ liter

Meat: $1.9 E 8$ kilogram

0.9

0.6

Meteorology Period of Record: 4 MAY 74 - 3 MAY 75 Recovery: $89 \%$

\section{SITE-SPECIFIC DATA - WATERBORNE PATHWAYS via MISSOURI RIVER}

Average River Flow at Site: $80,000 \mathrm{ft}^{3} / \mathrm{s}$

Drinking Water:

Exposed Population: None

Fish:

Edible Harvest: $1.0 \mathrm{E} 3 \mathrm{~kg} / \mathrm{yr}$

Dilution Factor: 1 


\section{POPULATION DOSE-COMMITMENT ESTIMATES AND \\ AVERAGE INDIVIDUAL DOSE-COMMITMENT HISTOGRAM FOR}

CALLAWAY

Dose Commitments (person-rem) from Waterborne Pathways

Total Body GI-LLI Ihyroid Bone

$\begin{array}{llllll}\text { Infant } & 0.0 \mathrm{E}+00 & 0.0 \mathrm{E}+00 & 0.0 \mathrm{E}+00 & 0.0 \mathrm{E}+00 & 0.0 \mathrm{E}+00 \\ \text { Child } & 9.2 \mathrm{E}-08 & 1.2 \mathrm{E}-07 & 1.1 \mathrm{E}-07 & 1.3 \mathrm{E}-08 & 1.0 \mathrm{E}-07 \\ \text { Teen } & 8.5 \mathrm{E}-08 & 1.4 \mathrm{E}-07 & 9.8 \mathrm{E}-08 & 8.0 \mathrm{E}-09 & 9.2 \mathrm{E}-08 \\ \text { Adult } & 6.9 \mathrm{E}-07 & 1.2 \mathrm{E}-06 & 7.5 \mathrm{E}-07 & 4.6 \mathrm{E}-08 & 7.1 \mathrm{E}-07 \\ \text { TOTAL } & 8.7 \mathrm{E}-07 & 1.5 \mathrm{E}-06 & 9.7 \mathrm{E}-07 & 6.7 \mathrm{E}-08 & 9.0 \mathrm{E}-07\end{array}$

Dose Commitments (person-rem) from Airborne Pathways

Total Body GI-LLI Thyroid Bone Liver Lung

$\begin{array}{lllllll}\text { Infant } & 1.6 \mathrm{E}-04 & 1.6 \mathrm{E}-04 & 2.2 \mathrm{E}-04 & 1.5 \mathrm{E}-04 & 1.6 \mathrm{E}-04 & 1.7 \mathrm{E}-04 \\ \text { Child } & 1.8 \mathrm{E}-03 & 1.8 \mathrm{E}-03 & 2.3 \mathrm{E}-03 & 1.7 \mathrm{E}-03 & 1.8 \mathrm{E}-03 & 2.0 \mathrm{E}-03 \\ \text { Teen } & 1.3 \mathrm{E}-03 & 1.3 \mathrm{E}-03 & 1.5 \mathrm{E}-03 & 1.2 \mathrm{E}-03 & 1.3 \mathrm{E}-03 & 1.6 \mathrm{E}-03 \\ \text { Adult } & 7.9 \mathrm{E}-03 & 7.9 \mathrm{E}-03 & 8.5 \mathrm{E}-03 & 7.5 \mathrm{E}-03 & 7.9 \mathrm{E}-03 & 8.7 \mathrm{E}-03 \\ \text { TOTAL } & 1.1 \mathrm{E}-02 & 1.1 \mathrm{E}-02 & 1.3 \mathrm{E}-02 & 1.1 \mathrm{E}-02 & 1.1 \mathrm{E}-02 & 1.2 \mathrm{E}-02\end{array}$

Production/Consumption factors:

Produce: 0.42

Milk: 2.9

Meat: 6.0

Fraction of Population Receiving an Average Individual Total-Body Dose Commitment from Airborne Pathways

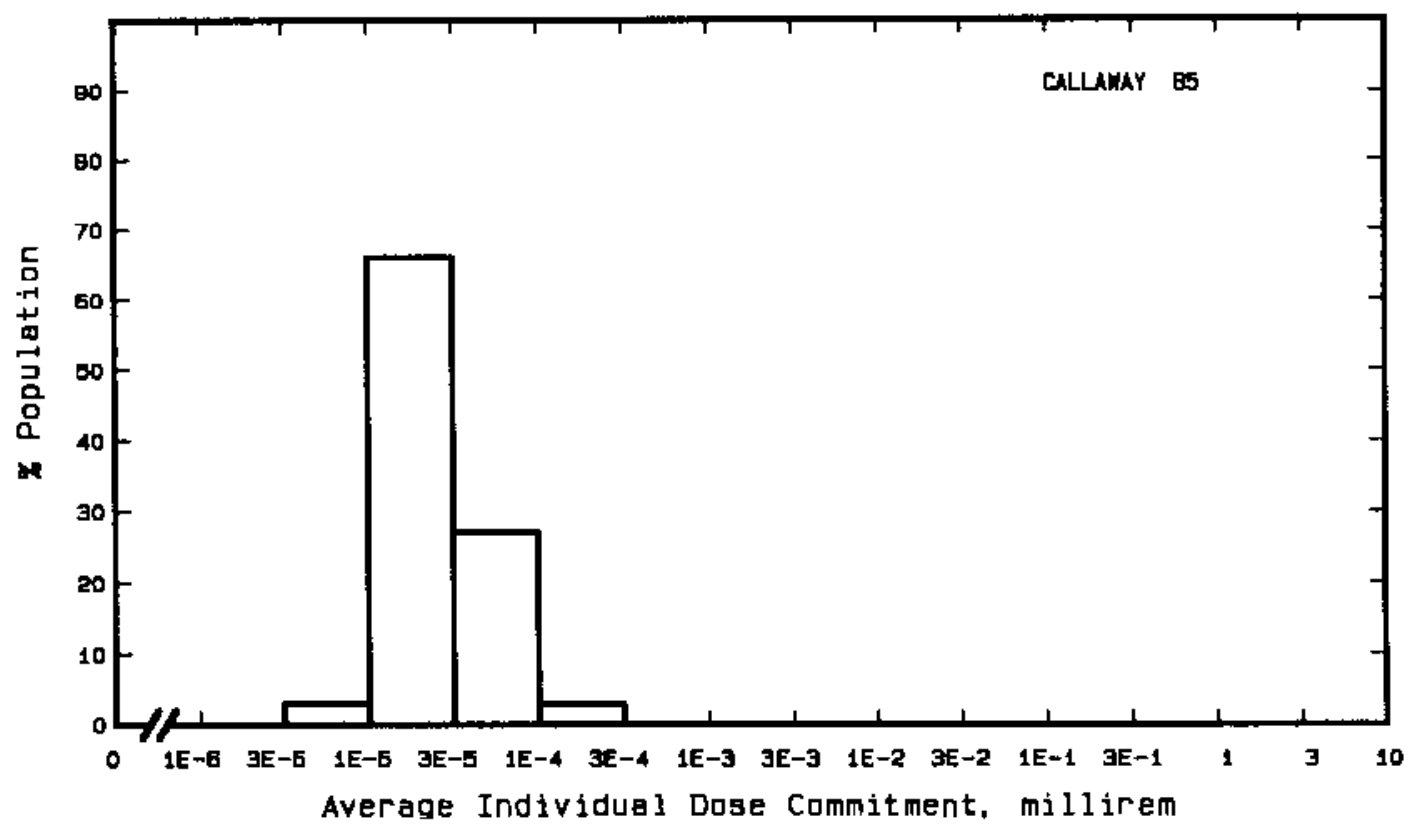


Site: CALVERT CLIFFS

LUSBY, MARYLAND

Location: N $38.4347^{\circ}$ W $76.4419^{\circ}$

POPULATION DATA

Total Population Within 2-to-80-km Region: 2.7E6

Major Metropolitan Centers Within Region:

Center

Population

Location

Washington, DC SMSA (3/4) $2,600,000$

Bowie

Annapolis

34,000

Waldorf

32,000

Sal isbury

9,800

16,000

Cambridge

12,000

$\begin{array}{lll}73 & \mathrm{~km} & \text { NW } \\ 71 & \mathrm{~km} & \text { NNW } \\ 61 & \mathrm{~km} & \mathrm{~N} \\ 46 & \mathrm{~km} & \text { WNW } \\ 75 & \mathrm{~km} & \text { E } \\ 32 & \mathrm{~km} & \text { ENE }\end{array}$

\section{SITE-SPECIFIC DATA - AIRBORNE PATHWAYS}

Average Annual State Production

of Crops and Animal Products

In $80-\mathrm{km}$ Radius Circle

Regional Productivity Factor:

Animal Grazing Factor:
Veg: $4.5 E 7$ kilogram

Milk: 5.0E8 liter

Meat: $6.2 E 7$ kilogram

Meteorology Period of Record: I JAN 75 - 31 DEC 75 Recovery: $96 \%$

SITE-SPECIFIC DATA - WATERBORNE PATHWAYS via CHESAPEAKE BAY

Average 0ilution $\mathrm{Flow}$

from Plant: $2,400 \mathrm{ft}^{3} / \mathrm{s}$

Fish:

Edible Harvest: $1.0 \mathrm{E} 7 \mathrm{~kg} / \mathrm{yr}$

Dilution Factor: $0.062(\mathrm{a})$

Invertebrates:

Edible Harvest: $7.4 \mathrm{E} 6 \mathrm{~kg} / \mathrm{yr}$

Dilution Factor: $0.062^{(a)}$

(a) Dilutions given in FES (1973). 
POPULATION DOSE-COMMITMENT ESTIMATES AND

AVERAGE INDIVIDUAL DOSE-COMMITMENT HISTOGRAM FOR

CALVERT CLIFFS 1 AND 2

Dose Commitments (person-rem) from Waterborne Pathways

Total Body GI-LLI $\underline{\text { Thyroid }}$ Bone Liver

$\begin{array}{llllll}\text { Infant } & 0.0 \mathrm{E}+00 & 0.0 \mathrm{E}+00 & 0.0 \mathrm{E}+00 & 0.0 \mathrm{E}+00 & 0.0 \mathrm{E}+00 \\ \text { Child } & 6.1 \mathrm{E}-02 & 1.1 \mathrm{E}+00 & 1.8 \mathrm{E}-01 & 1.1 \mathrm{E}-01 & 1.2 \mathrm{E}-01 \\ \text { Teen } & 6.5 \mathrm{E}-02 & 2.3 \mathrm{E}+00 & 1.3 \mathrm{E}-01 & 6.4 \mathrm{E}-02 & 1.1 \mathrm{E}-01 \\ \text { Adult } & 5.6 \mathrm{E}-01 & 2.0 \mathrm{E}+01 & 8.5 \mathrm{E}-01 & 3.7 \mathrm{E}-01 & 6.4 \mathrm{E}-01 \\ \text { TOTAL } & 6.8 \mathrm{E}-01 & 2.4 \mathrm{E}+01 & 1.2 \mathrm{E}+00 & 5.4 \mathrm{E}-01 & 8.7 \mathrm{E}-01\end{array}$

Dose Commitments (person-rem) from Airborne Pathways

Total Body GI-LLI Thyroid Bone Liver Lung

$\begin{array}{lllllll}\text { Infant } & 2.1 \mathrm{E}-03 & 2.0 \mathrm{E}-03 & 7.0 \mathrm{E}-02 & 2.3 \mathrm{E}-03 & 2.4 \mathrm{E}-03 & 2.2 \mathrm{E}-03 \\ \text { Child } & 2.3 \mathrm{E}-02 & 2.2 \mathrm{E}-02 & 3.8 \mathrm{E}-01 & 2.4 \mathrm{E}-02 & 2.4 \mathrm{E}-02 & 2.5 \mathrm{E}-02 \\ \text { Teen } & 1.6 \mathrm{E}-02 & 1.6 \mathrm{E}-02 & 1.6 \mathrm{E}-01 & 1.7 \mathrm{E}-02 & 1.7 \mathrm{E}-02 & 1.9 \mathrm{E}-02 \\ \text { Adult } & 9.8 \mathrm{E}-02 & 9.7 \mathrm{E}-02 & 5.6 \mathrm{E}-01 & 9.8 \mathrm{E}-02 & 1.0 \mathrm{E}-01 & 1.1 \mathrm{E}-01 \\ \text { TOTAL } & 1.4 \mathrm{E}-01 & 1.4 \mathrm{E}-01 & 1.2 \mathrm{E}+00 & 1.4 \mathrm{E}-01 & 1.4 \mathrm{E}-01 & 1.5 \mathrm{E}-01\end{array}$

Production/Consumption factors:

Produce: $0.051 \quad$ Milk: $0.84 \quad$ Meat: 0.17

Fraction of Population Aeceiving an Average Individual Total-Body Dose Cammitment from Airborne Pathways

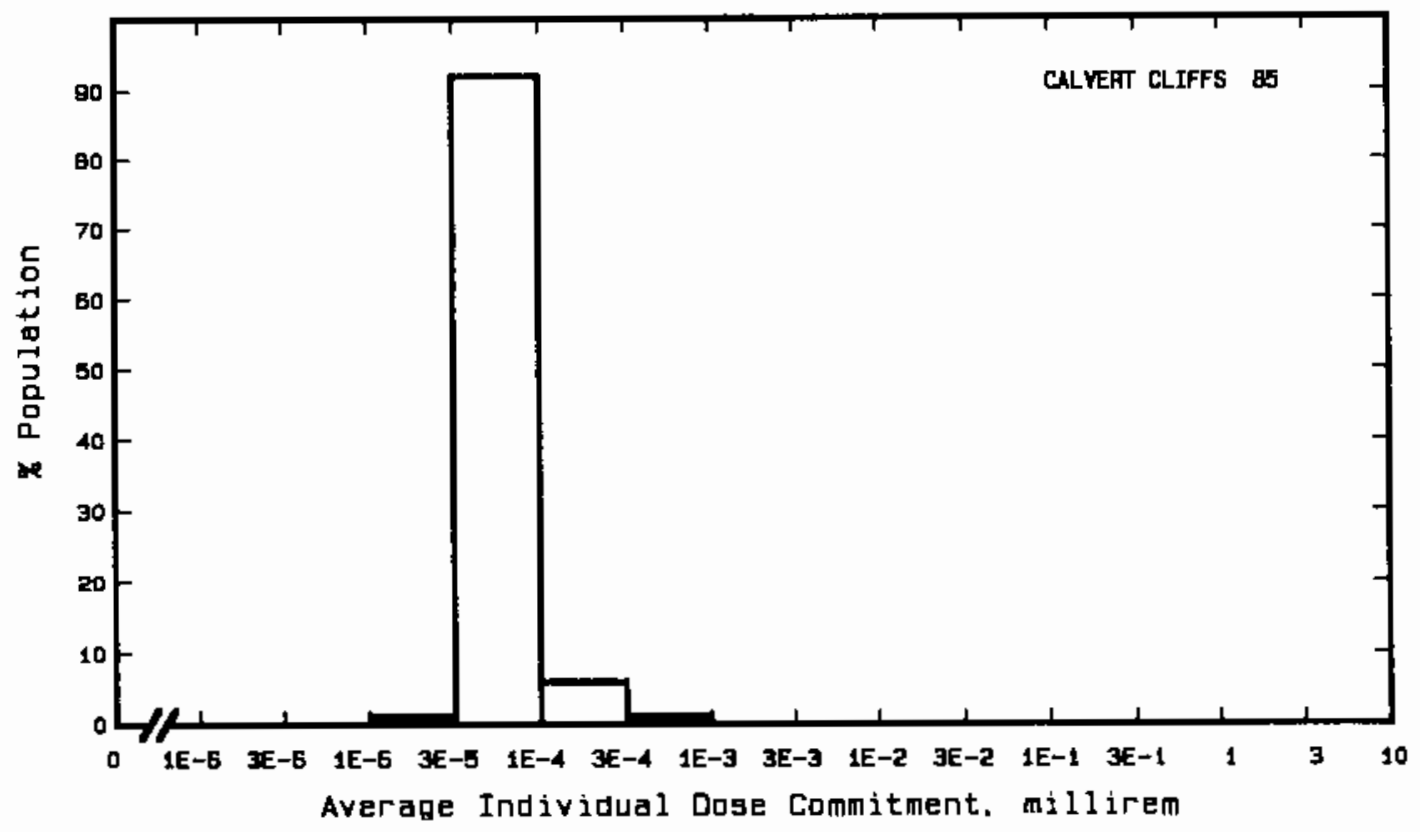


Site: CATAWBA

Location:

N $34.9950^{\circ}$

CLOVER, SOUTH CAROLINA

W $81.2450^{\circ}$

POPULATION DATA

Total Population Within 2-to-80-km Region: $1.5 E 6$

Major Metropolitan Centers Within Region:

Center

Charlotte-Gastonia SMSA

Kannapolis

Rock Hill

Spartanburg
Population

660,000

36,000

36,000

15,000
Location

$29 \mathrm{~km} \quad \mathrm{NE}$

$64 \mathrm{~km} \quad \mathrm{NE}$

$11 \mathrm{~km} \quad \mathrm{~S}$

$80 \mathrm{~km} \quad \mathrm{~W}$

\section{SITE-SPECIFIC DATA - AIRBORNE PATHWAYS}

Average Annual State Production

of Crops and Animal Products

In 80-km Radius Circle

Regional Productivity Factor:

Animal Grazing Factor:
Veg: 7.4E6 kilogram

Milk: 5.767 liter

Meat: 5.0E7 kilogram

0.9

0.75

Meteorology Period of Record: 17 DEC 75 - 16 DEC 76 Recovery: 94\%

SITE-SPECIFIC DATA - WATERBORNE PATHWAYS via CATAWBA RIVER

Average River Flow

at Site: $4,400 \mathrm{ft}^{3} / \mathrm{s}$

Drinking Water:

Exposed Population: None Dilution Factor:

Fish:

Edible Harvest: $1.0 \mathrm{E} 6 \mathrm{~kg} / \mathrm{yr}$

Dilution Factor: 1 


\section{POPULATION DOSE-COMMITMENT ESTIMATES AND AVERAGE INDIVIDUAL DOSE-COMMITMENT HISTOGRAM FOR \\ CATAWBA}

Dose Commitments (person-rem) from Waterborne Pathways

Jotal Body GI-LLI $\underline{\text { Thyroid }}$ Bone Liver

$\begin{array}{llllll}\text { Infant } & 0.0 \mathrm{E}+00 & 0.0 \mathrm{E}+00 & 0.0 \mathrm{E}+00 & 0.0 \mathrm{E}+00 & 0.0 \mathrm{E}+00 \\ \text { Child } & 3.4 \mathrm{E}-02 & 7.5 \mathrm{E}-02 & 7.0 \mathrm{E}-02 & 1.5 \mathrm{E}-01 & 1.8 \mathrm{E}-01 \\ \text { Teen } & 6.0 \mathrm{E}-02 & 1.6 \mathrm{E}-01 & 5.0 \mathrm{E}-02 & 9.3 \mathrm{E}-02 & 1.5 \mathrm{E}-01 \\ \text { Adult } & 6.3 \mathrm{E}-01 & 1.4 \mathrm{E}+00 & 3.3 \mathrm{E}-01 & 5.4 \mathrm{E}-01 & 8.8 \mathrm{E}-01 \\ \text { TOTAL } & 7.2 \mathrm{E}-01 & 1.6 \mathrm{E}+00 & 4.5 \mathrm{E}-01 & 7.8 \mathrm{E}-01 & 1.2 \mathrm{E}+00\end{array}$

Dose Commitments (person-rem) from Airborne Pathways

Total Body GI-LLI Thyroid Bone Liver Lung

$\begin{array}{lllllll}\text { Infant } & 2.9 \mathrm{E}-04 & 2.9 \mathrm{E}-04 & 5.3 \mathrm{E}-04 & 2.9 \mathrm{E}-04 & 2.9 \mathrm{E}-04 & 3.1 \mathrm{E}-04 \\ \text { Child } & 3.2 \mathrm{E}-03 & 3.2 \mathrm{E}-03 & 4.8 \mathrm{E}-03 & 3.2 \mathrm{E}-03 & 3.2 \mathrm{E}-03 & 3.5 \mathrm{E}-03 \\ \text { Teen } & 2.4 \mathrm{E}-03 & 2.4 \mathrm{E}-03 & 3.1 \mathrm{E}-03 & 2.3 \mathrm{E}-03 & 2.4 \mathrm{E}-03 & 2.7 \mathrm{E}-03 \\ \text { Adult } & 1.4 \mathrm{E}-02 & 1.4 \mathrm{E}-03 & 1.7 \mathrm{E}-02 & 1.4 \mathrm{E}-02 & 1.4 \mathrm{E}-02 & 1.5 \mathrm{E}-02 \\ \text { TOTAL } & 2.0 \mathrm{E}-02 & 2.0 \mathrm{E}-02 & 2.6 \mathrm{E}-02 & 2.0 \mathrm{E}-02 & 2.0 \mathrm{E}-02 & 2.2 \mathrm{E}-02\end{array}$

Production/Consumption factors:

Produce: 0.022 Milk: $0.26 \quad$ Meat: 0.37

Fraction of Population Receiving an Average Individual Total-Body Dose Commitment from Airborne Pathways

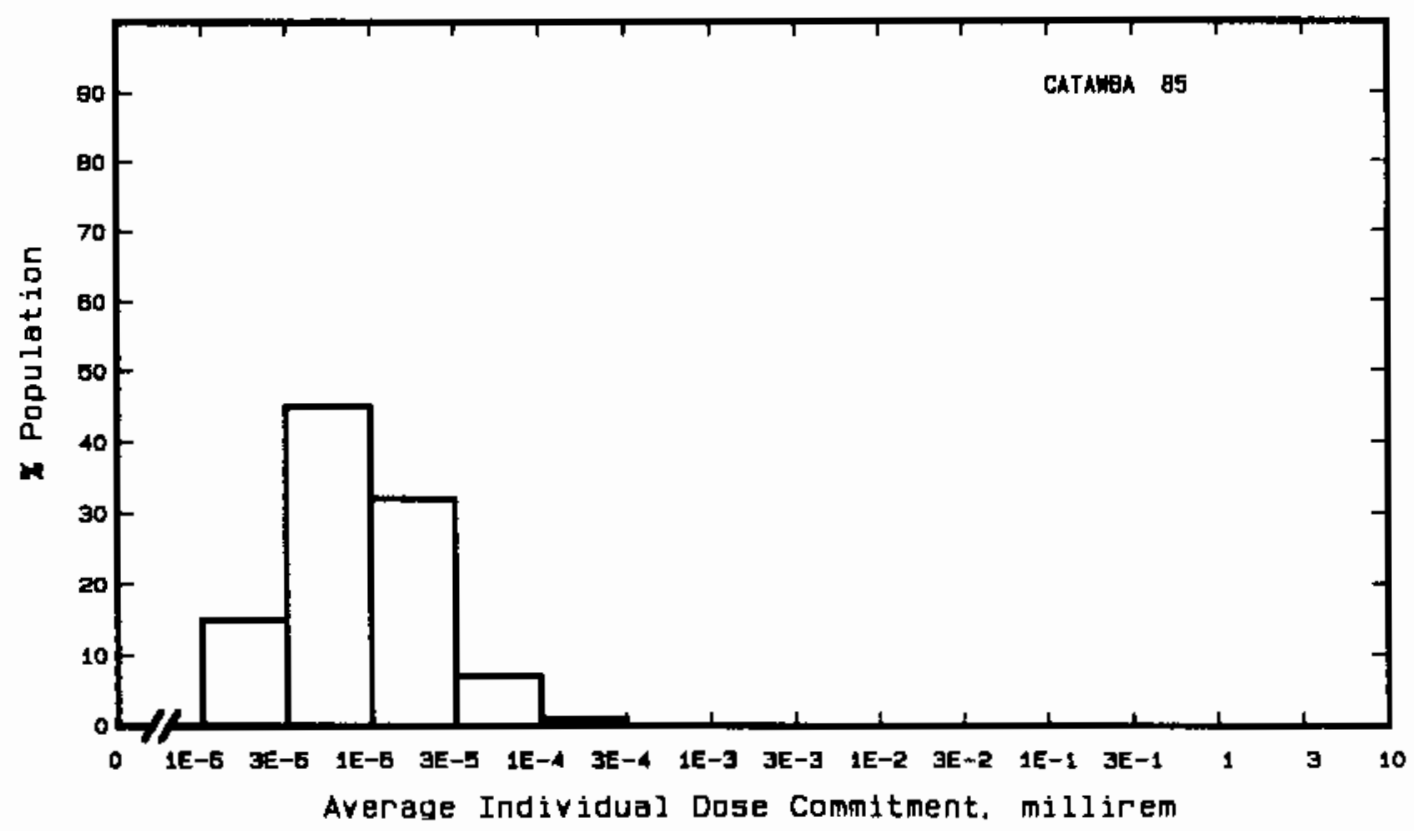


Site: COOK

BENTON HARBOR, MICHIGAN

Location: $\quad \mathrm{N} 41.9761^{\circ} \quad$ W $86.5664^{\circ}$

POPULATION DATA

Total Poputation Within 2-to-80-km Region: 1.1E6

Major Metropolitan Centers Within Region:

\begin{tabular}{lccc}
\multicolumn{1}{c}{ Center } & Population & \multicolumn{2}{c}{ Location } \\
\cline { 2 - 4 } & & & \\
Gary & 140,000 & $77 \mathrm{~km}$ & SW \\
South Bend SMSA & 280,000 & $42 \mathrm{~km}$ & SE \\
Elkhart SMSA & 140,000 & $5 \mathrm{Bm}$ & SE \\
Mishiwaka & 40,000 & $47 \mathrm{~km}$ & SE \\
Michigan City & 37,000 & $40 \mathrm{~km}$ & SW
\end{tabular}

SITE-SPECIFIC DATA - AIRBORNE PATHWAYS

Average Annual State Production

of Crops and Animal Products

In $80-\mathrm{km}$ Radius Circle

Regional Productivity Factor:

Animal Grazing Factor:
Veg: 1.lE8 kilogram

Milk: 2.3E8 liter

Meat: $1.9 \mathrm{E} 8 \mathrm{kilogram}$

0.6

0.5

Meteorology Period of Record: 1 MAY 75 - 31 APR 76 Recovery: 95\%

SITE-SPECIFIC DATA - WATERBORNE PATHWAYS via LAKE MICHIGAN

Average Dilution Flow

from Plant: $2,500 \mathrm{ft}^{3} / \mathrm{s}$

Drinking Water:

Exposed Population: 250,000

Dilution Factor: $0.025(a)$

Fish:

Edible Harvest: $1.5 \mathrm{E} 6 \mathrm{~kg} / \mathrm{yr}$

Dilution Factor: 0.01

(a) Drinking water dilution factor estimated by averaging dilution factors derived from FES (1973) suitably weighted for population. 


\section{POPULATION DOSE-COMHITMENT ESTIMATES AND \\ AVERAGE INDIVIDUAL DOSE-COMMITMENT HISTOGRAM FOR}

COOK 1 AND 2

Dose Commitments (person-rem) from Waterborne Pathways

Total Body

$$
\underline{G I-L L I}
$$

Thyroid

Bone

Liver

Infant

$2.6 \mathrm{E}-03$

2. $5 \mathrm{E}-03$

4.7E-03

$9.6 \mathrm{E}-04$

3.7E-03

Child

$4,4 \mathrm{E}-02$

3.7E-02

$4.4 \mathrm{E}-02$

7. $1 \mathrm{E}-02$

1. $1 \mathrm{E}-01$

Teen

$3.8 \mathrm{E}-02$

$2.8 \mathrm{E}-02$

$1.5 \mathrm{E}-02$

3. $9 \mathrm{E}-02$

7.7E-02

Adult

$3.9 E-01$

2.5E-01

1.2E-01

2.3E-01

4. $9 \mathrm{E}-01$

TOTAL

4.7E-01

3.1E-01

1.9E-01

$3.4 \mathrm{E}-01$

$6.8 \mathrm{E}-01$

Dose Commitments (person-rem) from Airborne Pathways

Tota] Body GI-LLI Thyroid Bone Liver

$\begin{array}{lllllll}\text { Infant } & 9.9 \mathrm{E}-04 & 8.8 \mathrm{E}-04 & 5.1 \mathrm{E}-02 & 1.4 \mathrm{E}-03 & 1.6 \mathrm{E}-03 & 1.1 \mathrm{E}-03 \\ \text { Child } & 1.2 \mathrm{E}-02 & 1.1 \mathrm{E}-02 & 3.5 \mathrm{E}-01 & 1.5 \mathrm{E}-02 & 1.6 \mathrm{E}-02 & 1.2 \mathrm{E}-02 \\ \text { Teen } & 8.4 \mathrm{E}-03 & 8.2 \mathrm{E}-03 & 1.4 \mathrm{E}-01 & 8.4 \mathrm{E}-03 & 1.0 \mathrm{E}-02 & 9.5 \mathrm{E}-03 \\ \text { Adult } & 5.0 \mathrm{E}-02 & 5.1 \mathrm{E}-02 & 4.8 \mathrm{E}-01 & 4.6 \mathrm{E}-02 & 5.2 \mathrm{E}-02 & 5.1 \mathrm{E}-02 \\ \text { TOTAL } & 7.1 \mathrm{E}-02 & 7.1 \mathrm{E}-02 & 1.0 \mathrm{E}+00 & 7.0 \mathrm{E}-02 & \mathrm{~B} .0 \mathrm{E}-02 & 7.4 \mathrm{E}-02\end{array}$

Production/Consumption factors:

Produce: 0.30

Milk: 0.93

Meat: 1.3

Fraction of Population Receiving an Average Individual Total-Body Dose Commitment from Airborne Pathways

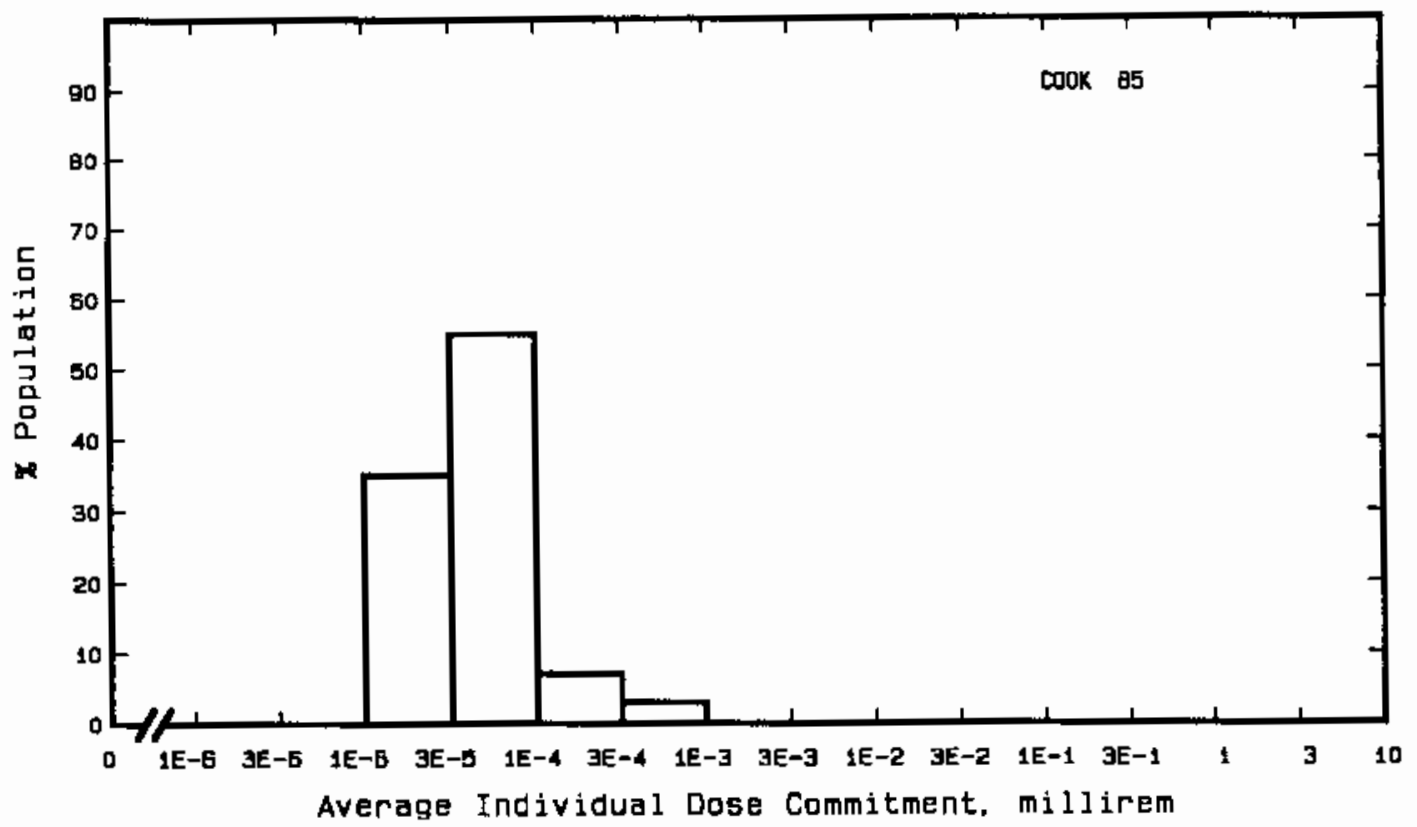


Site: COOPER

Location: N $40.3619^{\circ}$
NEMAHA COUNTY, NEBRASKA

W $95.6411^{\circ}$

POPULATION DATA

Total Population Within 2-to-80-km Region: 1.7E5

Major Metropolitan Centers Within Region:

Center

Nebraska City

Red Oak

Plattsmouth

Shenandoah

Falls City
Population

7,100

6,800

6,300

6,300

5,400
Location

$40 \mathrm{~km} \quad \mathrm{NNW}$

$80 \mathrm{~km} \quad \mathrm{NNE}$

$76 \mathrm{~km} \quad \mathrm{NNW}$

$51 \mathrm{~km} \quad \mathrm{NNE}$

$33 \mathrm{~km} \mathrm{~S}$

SITE-SPECIFIC DATA - AIRBORNE PATHWAYS

Average Annual State Production

of Crops and Animal Products

In $80-\mathrm{km}$ Radius Circle

Regional Productivity Factor:

Animal Grazing Factor:
Veg: $9.7 \mathrm{E} 7$ kilogram

Milk: 7.2E7 liter

Meat: 2.0EB kilogram

1

0.6

Meteorology Period of Record: 1 MAR 70 - 31 DEC 75 Recovery: 89\%

SITE-SPECIFIC DATA - WATERBDRNE PATHWAYS via MISSOURI RIVER

Average River Flow at Site: $31,000 \mathrm{ft}^{3} / \mathrm{s}$

Drinking Water:

Exposed Population: None

Fish:

Edible Harvest: $5.0 \mathrm{E}_{3}(\mathrm{a}) \mathrm{kg} / \mathrm{yr}$

Dilution Factor: $0.5^{(a)}$

(a) Assumes $1 / 2$ fish harvest caught below plant. 


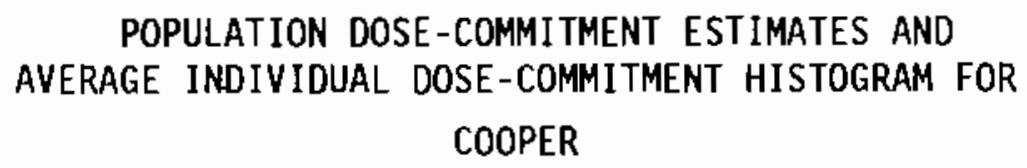

Dose Commitments (person-rem) from Waterborne Pathways

Total Body GI-LLI $\underline{\text { Thyroid Bone Liver }}$

$\begin{array}{llllll}\text { Infant } & 0.0 \mathrm{E}+00 & 0.0 \mathrm{E}+00 & 0.0 \mathrm{E}+00 & 0.0 \mathrm{E}+00 & 0.0 \mathrm{E}+00 \\ \text { Child } & 2.4 \mathrm{E}-03 & 2.1 \mathrm{E}-04 & 3.8 \mathrm{E}-06 & 1.1 \mathrm{E}-02 & 1.3 \mathrm{E}-02 \\ \text { Teen } & 4.6 \mathrm{E}-03 & 4.2 \mathrm{E}-04 & 2.8 \mathrm{E}-06 & 6.7 \mathrm{E}-03 & 1.1 \mathrm{E}-02 \\ \text { Adult } & 4.9 \mathrm{E}-02 & 3.7 \mathrm{E}-03 & 1.8 \mathrm{E}-05 & 3.9 \mathrm{E}-02 & 6.7 \mathrm{E}-02 \\ \text { TOTAL } & 5.6 \mathrm{E}-02 & 4.3 \mathrm{E}-03 & 2.5 \mathrm{E}-05 & 5.7 \mathrm{E}-02 & 9.2 \mathrm{E}-02\end{array}$

Dose Commitments (person-rem) from Airborne Pathways

Total Body GI-LLI Thyroid Bone Liver Lung

$\begin{array}{lllllll}\text { Infant } & 1.8 \mathrm{E}-04 & 1.8 \mathrm{E}-04 & 4.9 \mathrm{E}-04 & 1.9 \mathrm{E}-04 & 1.9 \mathrm{E}-04 & 2.1 \mathrm{E}-04 \\ \text { Child } & 2.2 \mathrm{E}-03 & 2.2 \mathrm{E}-03 & 5.0 \mathrm{E}-03 & 3.1 \mathrm{E}-03 & 2.1 \mathrm{E}-03 & 2.4 \mathrm{E}-03 \\ \text { Teen } & 1.6 \mathrm{E}-03 & 1.7 \mathrm{E}-03 & 2.6 \mathrm{E}-03 & 1.9 \mathrm{E}-03 & 1.5 \mathrm{E}-03 & 1.9 \mathrm{E}-03 \\ \text { Adult } & 9.3 \mathrm{E}-03 & 1.0 \mathrm{E}-02 & 1.3 \mathrm{E}-02 & 1.0 \mathrm{E}-02 & 9.1 \mathrm{E}-03 & 1.0 \mathrm{E}-03 \\ \text { TOTAL } & 1.3 \mathrm{E}-02 & 1.4 \mathrm{E}-02 & 2.1 \mathrm{E}-02 & 1.6 \mathrm{E}-02 & 1.3 \mathrm{E}-02 & 1.5 \mathrm{E}-02\end{array}$

Production/Consumption factors:

Produce: 2.9 Milk: $3.2 \quad$ Meat: 14

Fraction of Population Receiving an Average Individual Total-Body Dose Commitment from Airoorne Pathways

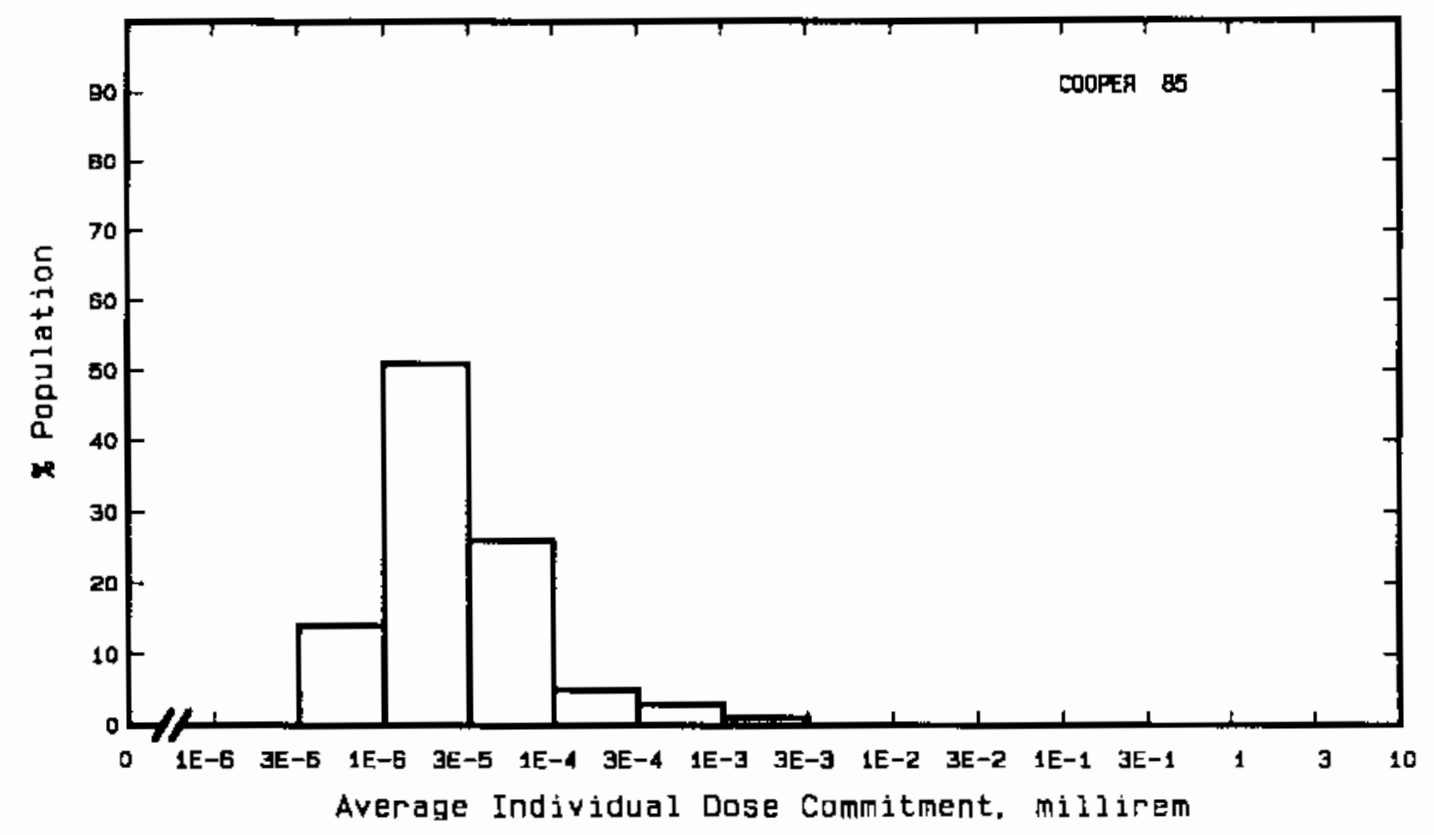


Site: CRYSTAL RIVER

CRYSTAL RIVER, FLORIDA

Location: $\quad$ N $28.9572^{\circ} \quad$ W $82.6989^{\circ}$

POPULATIDN DATA

Total Population Within 2-to-80-km Region: 4.3E5

Major Metropolitan Centers Within Region:

Center Population Location

$\begin{array}{llll}\text { Ocala } & 44,000 & 60 \mathrm{~km} & \text { ENE } \\ \text { Leesburg } & 15,000 & 80 \mathrm{~km} & \mathrm{E} \\ \text { New Port Richey } & 13,000 & 79 \mathrm{~km} & \mathrm{~S}\end{array}$

SITE-SPECIFIC DATA - AIRBORNE PATHWAYS

Average Annual State Production

of Crops and Animal Products

In 80-km Radius Circle

Regional Productivity Factor:

Animal Grazing Factor:
Veg: 2.8E7 kilogram

Milk: $1.1 E 8$ liter

Meat: $7.2 E 7$ kilogram

0.5

1

Meteorology Period of Record: 1 JAN 75 - 31 DEC 75 Recovery: $93 \%$

SITE-SPECIFIC DATA - WATERBORNE PATHWAYS via GULF Of MEXICO

Average Dilution Flow

from Plant: $26 \mathrm{ft}^{3} / \mathrm{s}$

Fish:

Edible Harvest: $3.2 \mathrm{E} 5 \mathrm{~kg} / \mathrm{yr}$

Dilution Factor: 0.1

Invertebrates:

Edible Harvest: $1.8 \mathrm{E} 5 \mathrm{~kg} / \mathrm{yr}$

Dilution Factor: 0.1 


\section{POPULATION DOSE-COMMITMENT ESTIMATES AND \\ AVERAGE INDIVIDUAL DOSE-COMMITMENT HISTOGRAM FOR}

CRYSTAL RIVER

Dose Commitments (person-rem) from Waterborne Pathways

Total Body

$\underline{G I-L L I}$

Thyroid

Bone

Liver

Infant

$0.0 \mathrm{E}+00$

$0.0 E+00$

$0.0 \mathrm{E}+00$

$0.0 \mathrm{E}+00$

$0.0 \mathrm{E}+00$

Child

7. $0 \mathrm{E}-01$

$1.4 \mathrm{E}+00$

5.1E-02

7.0E-01

$1.0 \mathrm{E}+00$

Teen

4. $9 \mathrm{E}-01$

3. $0 \mathrm{E}+00$

$3.7 \mathrm{E}-02$

4.3E-01

8.7E-01

Adult

3. $1 \mathrm{E}+00$

2. $6 \mathrm{E}+01$

2.5E-01

2. $7 \mathrm{E}+00$

5. $3 \mathrm{E}+00$

TOTAL

4. $3 \mathrm{E}+00$

3. $1 E+01$

3.3E-01

3. $8 \mathrm{E}+00$

7. $1 \mathrm{E}+00$

Dose Commitments (person-rem) from Airborne Pathways

\section{Total Body GI-LLI Ihyroid Bone Liver Lung}

$\begin{array}{lllllll}\text { Infant } & 1.3 \mathrm{E}-03 & 6.4 \mathrm{E}-04 & 7.8 \mathrm{E}-04 & 3.5 \mathrm{E}-03 & 6 . \mathrm{IE}-04 & 7.1 \mathrm{E}-04 \\ \text { Child } & 2.9 \mathrm{E}-02 & 8.1 \mathrm{E}-03 & 7.7 \mathrm{E}-03 & 1.0 \mathrm{E}-01 & 6.8 \mathrm{E}-03 & 8.3 \mathrm{E}-03 \\ \text { Teen } & 1.4 \mathrm{E}-02 & 6.1 \mathrm{E}-03 & 5.2 \mathrm{E}-03 & 4.9 \mathrm{E}-02 & 4.9 \mathrm{E}-03 & 6.3 \mathrm{E}-03 \\ \text { Adult } & 7.1 \mathrm{E}-02 & 3.5 \mathrm{E}-02 & 3.0 \mathrm{E}-02 & 2.3 \mathrm{E}-01 & 3.0 \mathrm{E}-02 & 3.4 \mathrm{E}-02 \\ \text { TOTAL } & 1.2 \mathrm{E}-01 & 5.0 \mathrm{E}-02 & 4.4 \mathrm{E}-02 & 3.8 \mathrm{E}-01 & 4.2 \mathrm{E}-02 & 4.9 \mathrm{E}-02\end{array}$

Production/Consumption factors:
Produce: 0.17
Milk: 1.0
Meat: 1.1

Fraction of Population Receiving an Average Individual Total-Body Dose Commitment from Airborne Pathways

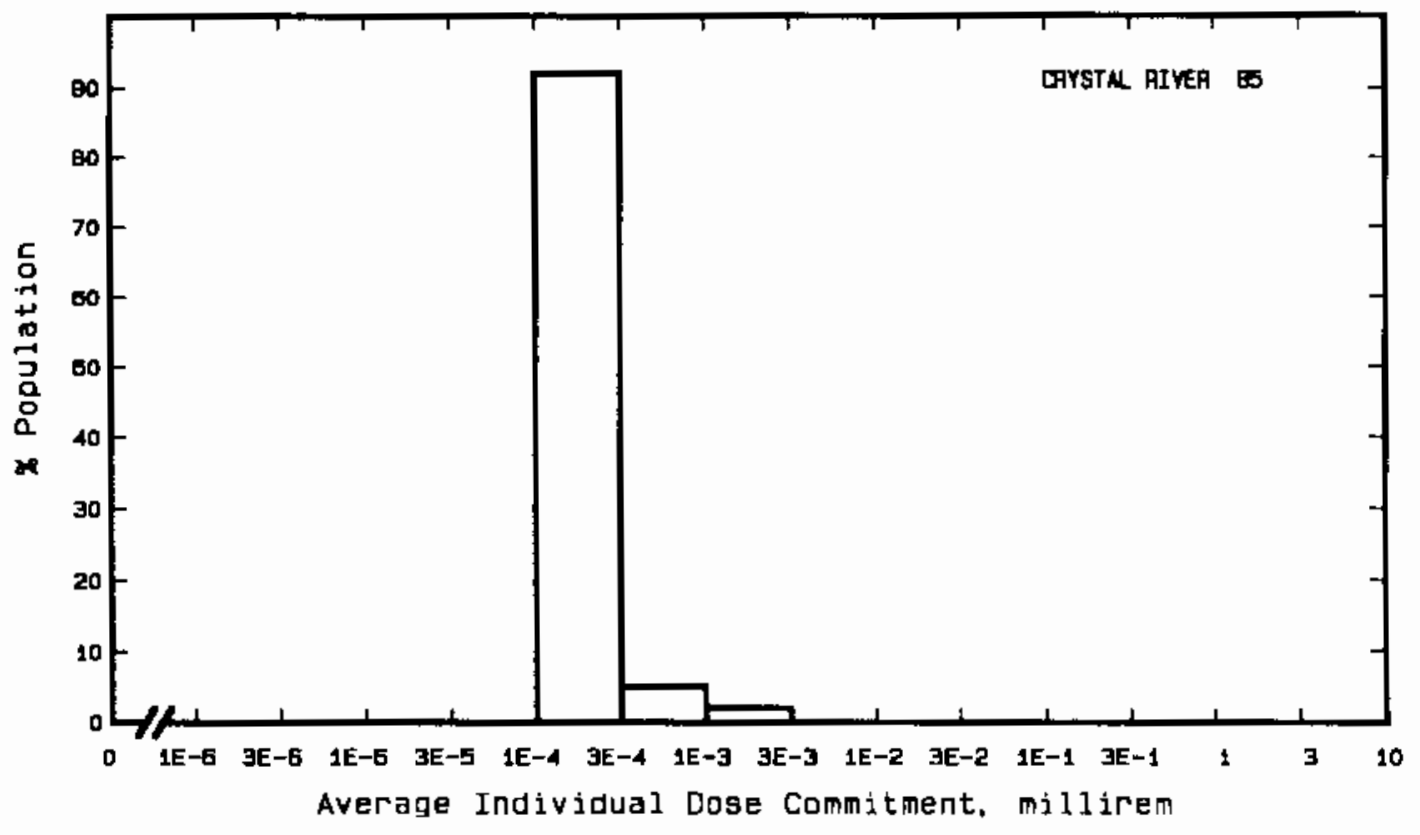


Site: DAVIS-BESSE

PORT CLINTON, OHIO

Location: $\quad N 41.5972^{\circ} \quad$ W $83.0864^{\circ}$

POPULATION DATA

Tota] Population Within 2-to-80-km Region: $1.8 \mathrm{E} 6$

Major Metropolitan Centers Within Region:

Center

Toledo SMSA

Dearborn

Taylor

Lorain

Lincoln Park

Findlay

Sandusky
Population

610,000

91,000

77,000

75,000

45,000

36,000

33,000
Location

$38 \mathrm{~km} \quad W W$

$80 \mathrm{~km} \mathrm{~N}$

$71 \mathrm{~km} \mathrm{~N}$

$77 \mathrm{~km} \quad$ ESE

$73 \mathrm{~km} \quad \mathrm{~N}$

$77 \mathrm{~km} \quad \mathrm{SW}$

$35 \mathrm{~km} \quad$ ESE

\section{SITE-SPECIFIC DATA - AIRBORNE PATHWAYS}

Average Annual State Production

of Crops and Animal Products

In 80-km Radius Circle

Regional Productivity Factor:

Animal Grazing Factor:
Veg: $6.9 \mathrm{E} 7 \mathrm{kilogram}$

Milk: 3.7E8 liter

Meat: 1.2E8 kilogram

Meteorology Period of Record: 4 AUG 74 - 3 AUG 76 Recovery: 99\%

SITE-SPECIFIC DATA - WATERBORNE PATHWAYS via LAKE ERIE

Average Dilution Flow

from Plant: $30 \mathrm{ft}^{3} / \mathrm{s}$

Drinking Water:

Exposed Population: 450,000

Dilution Factor: $1.6 \mathrm{E}-4(\mathrm{a})$

Fish:

Edible Harvest: $5.7 \mathrm{E}^{(\mathrm{b})} \mathrm{kg} / \mathrm{yr}$

Dilution Factor: $1.8 \mathrm{E}-4^{(b)}$

(a) Drinking water dilution factor estimated by averaging dilution factor derived from FES (1973), suitably weighted for population.

(b) Letter from Terry D. Murray, Toledo Edison Company to Charles A. Willis, NRC, July 20, 1984. 
POPULATION DOSE-COMMITMENT ESTIMATES AND

AVERAGE INDIVIDUAL DOSE-COMMITMENT HISTOGRAM FOR

DAVIS-BESSE

Dose Commitments (person-rem) from Waterborne Pathways

Total Body GI-LLI $\underline{\text { Thyroid }}$ Bone

$\begin{array}{llllll}\text { Infant } & 1.7 \mathrm{E}-04 & 1.5 \mathrm{E}-04 & 1.4 \mathrm{E}-04 & 2.5 \mathrm{E}-04 & 4.6 \mathrm{E}-04 \\ \text { ChiTd } & 2.2 \mathrm{E}-02 & 2.7 \mathrm{E}-03 & 1.6 \mathrm{E}-03 & 1.1 \mathrm{E}-01 & 1.2 \mathrm{E}-01 \\ \text { Teen } & 3.9 \mathrm{E}-02 & 2.5 \mathrm{E}-03 & 6.2 \mathrm{E}-04 & 6.5 \mathrm{E}-02 & 1.0 \mathrm{E}-01 \\ \text { Adult } & 4.3 \mathrm{E}-01 & 2.1 \mathrm{E}-02 & 5.2 \mathrm{E}-03 & 3.7 \mathrm{E}-01 & 6.0 \mathrm{E}-01 \\ \text { TOTAL } & 4.9 \mathrm{E}-01 & 2.6 \mathrm{E}-02 & 7.6 \mathrm{E}-03 & 5.5 \mathrm{E}-01 & 8.2 \mathrm{E}-01\end{array}$

Dose Commitments (person-rem) from Airborne Pathways

Total Body GI-LLI Thyroid Bone Liver Lung

$\begin{array}{lllllll}\text { Infant } & 9.5 \mathrm{E}-05 & 9.4 \mathrm{E}-05 & 5.7 \mathrm{E}-04 & 2.4 \mathrm{E}-05 & 9.6 \mathrm{E}-05 & 9.6 \mathrm{E}-05 \\ \text { Child } & 1.2 \mathrm{E}-03 & 1.2 \mathrm{E}-03 & 3.8 \mathrm{E}-03 & 2.6 \mathrm{E}-04 & 1.2 \mathrm{E}-03 & 1.2 \mathrm{E}-03 \\ \text { Teen } & 7.4 \mathrm{E}-04 & 7.4 \mathrm{E}-04 & 1.7 \mathrm{E}-03 & 1.9 \mathrm{E}-04 & 7.5 \mathrm{E}-04 & 7.8 \mathrm{E}-04 \\ \text { Adult } & 4.1 \mathrm{E}-03 & 4.1 \mathrm{E}-03 & 7.2 \mathrm{E}-03 & 1.1 \mathrm{E}-03 & 4.1 \mathrm{E}-03 & 4.2 \mathrm{E}-03 \\ \text { TOTAL } & 6.1 \mathrm{E}-03 & 6.1 \mathrm{E}-03 & 1.3 \mathrm{E}-02 & 1.6 \mathrm{E}-03 & 6.1 \mathrm{E}-03 & 6.3 \mathrm{E}-03\end{array}$

Production/Consumption factors:

Produce: $0.12 \quad$ Milk: $0.96 \quad$ Meat: 0.48

Fraction of Population Receiving an Average Individual Total-Body Dose Commitment from Airborne Pathways

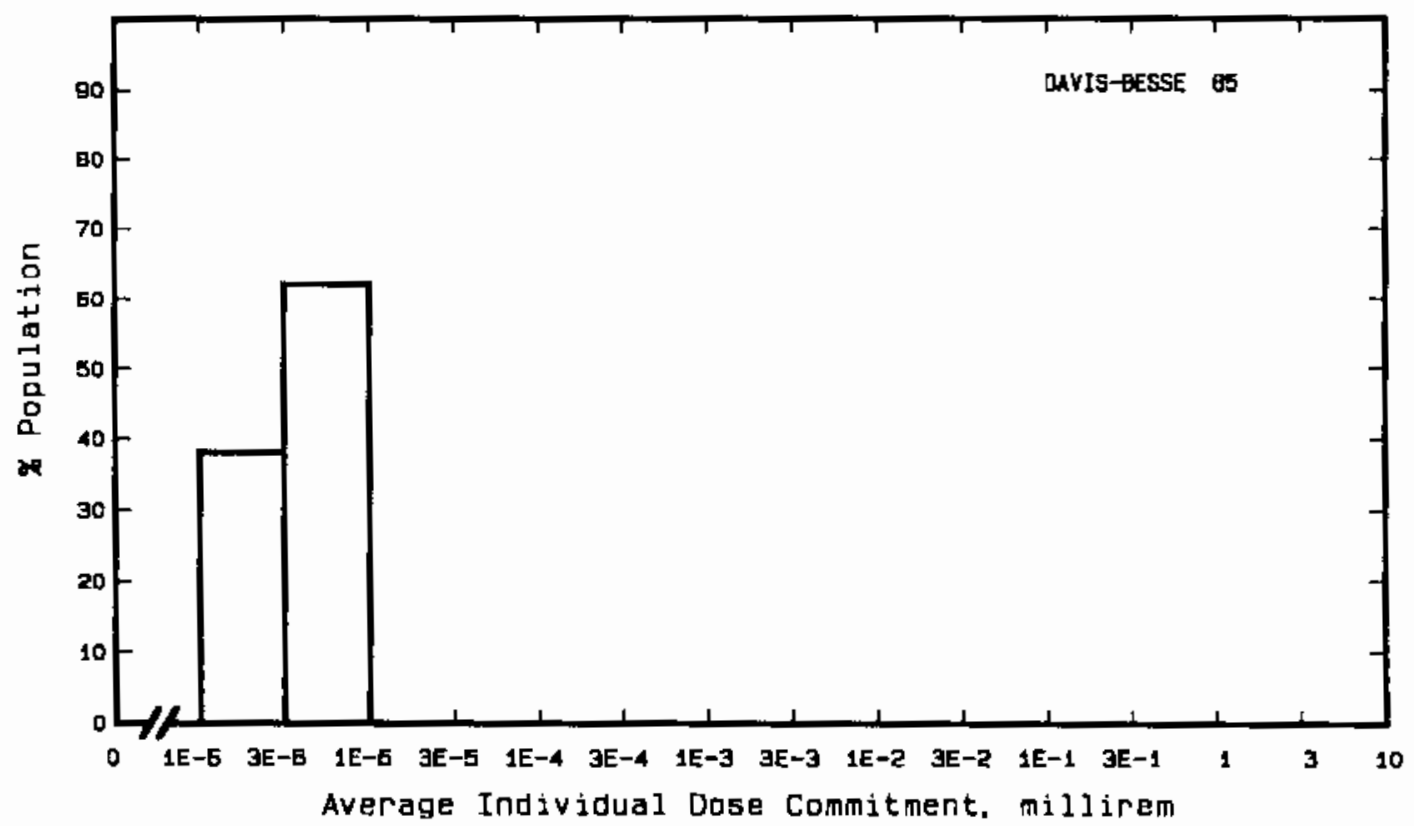


Site: DIABLO CANYON

AVILA BEACH, CALIFORNIA

Location: $\quad$ N $35.2111^{\circ} \quad$ W $120.8522^{\circ}$

POPULATION DATA

Total Population Within 2-to-80-km Region: 2.9E5

Major Metropolitan Centers Within Region:

Center

San Luis Obispo

Atascudero

Lompoc

Morro Bay
Population

38,000

13,000

26,000

9,400
Location

$19 \mathrm{~km}$ ENE

$34 \mathrm{~km}$ NNE

$74 \mathrm{~km} \quad$ SSE

$18 \mathrm{~km} \mathrm{~N}$

SITE-SPECIFIC DATA - AIRBORNE PATHWAYS

Average Annual State Production

of Crops and Animal Products

In $80-\mathrm{km}$ Radius Circle

Regional Productivity Factor:

Animal Grazing Factor:
Veg: $4.8 E 7$ kilogram

Milk: 2.3E8 liter

Meat: $5.0 E 7$ kilogram

0.5

1

Meteorology Period of Record: 1 JAN 84 - 31 DEC 84 Recovery: 98\%

SITE-SPECIFIC DATA - WATERBORNE PATHWAYS via Pacific Ocean

Average Dilution Flow

from Plant: $670 \mathrm{ft}^{3} / \mathrm{s}$

Fish:

Edible Harvest: $2.0 \mathrm{E} 6 \mathrm{~kg} / \mathrm{yr}$

Dilution Factor: 0.001

Invertebrates:

Edible Harvest: None 


\section{POPULATION DOSE-COMMITMENT ESTIMATES AND AVERAGE INDIVIDUAL DOSE-COMMITTMENT HISTOGRAM FOR}

OIABLO CANYON 1 AND 2

Dose Commitments (person-rem) from Waterborne Pathways

Total Body GI-LLI Thyroid Bone Liver

$\begin{array}{llllll}\text { Infant } & 0.0 \mathrm{E}+00 & 0.0 \mathrm{E}+00 & 0.0 \mathrm{E}+00 & 0.0 \mathrm{E}+00 & 0.0 \mathrm{E}+00 \\ \text { Child } & 6.0 \mathrm{E}-04 & 1.2 \mathrm{E}-03 & 1.3 \mathrm{E}-04 & 3.9 \mathrm{E}-04 & 7.3 \mathrm{E}-04 \\ \text { Teen } & 4.2 \mathrm{E}-04 & 2.5 \mathrm{E}-03 & 9.7 \mathrm{E}-05 & 2.4 \mathrm{E}-04 & 6.5 \mathrm{E}-04 \\ \text { Adu1t } & 2.5 \mathrm{E}-03 & 2.2 \mathrm{E}-02 & 6.6 \mathrm{E}-04 & 1.4 \mathrm{E}-03 & 3.9 \mathrm{E}-03 \\ \text { TOTAL } & 3.6 \mathrm{E}-03 & 2.6 \mathrm{E}-02 & 8.9 \mathrm{E}-04 & 2.0 \mathrm{E}-03 & 5.3 \mathrm{E}-03\end{array}$

Dose Commitments (person-rem) from Airborne Pathways

Total Body GI-LLI Ihyroid Bone Liver Lung

$\begin{array}{lllllll}\text { Infant } & 9.5 \mathrm{E}-05 & 9.5 \mathrm{E}-05 & 1.7 \mathrm{E}-04 & 7.7 \mathrm{E}-05 & 9.5 \mathrm{E}-05 & 1.0 \mathrm{E}-04 \\ \text { Child } & 1.2 \mathrm{E}-03 & 1.2 \mathrm{E}-03 & 1.6 \mathrm{E}-03 & 8.6 \mathrm{E}-04 & 1.2 \mathrm{E}-03 & 1.3 \mathrm{E}-03 \\ \text { Teen } & 8.2 \mathrm{E}-04 & 8.2 \mathrm{E}-04 & 1.0 \mathrm{E}-03 & 6.3 \mathrm{E}-04 & 8.2 \mathrm{E}-04 & 9.4 \mathrm{E}-04 \\ \text { Adult } & 4.9 \mathrm{E}-03 & 4.9 \mathrm{E}-03 & 5.5 \mathrm{E}-03 & 3.8 \mathrm{E}-03 & 4.9 \mathrm{E}-03 & 5.3 \mathrm{E}-03 \\ \text { TOTAL } & 7.0 \mathrm{E}-03 & 7.0 \mathrm{E}-03 & 8.3 \mathrm{E}-03 & 5.4 \mathrm{E}-03 & 7.0 \mathrm{E}-03 & 7.6 \mathrm{E}-03\end{array}$

Production/Consumption factors:

Produce: 0.42 Milk: $3.1 \quad$ Meat: 1.1

Fraction of Population Receiving an Average Individual Total-Body Dose Commitment from Airborne Pathways

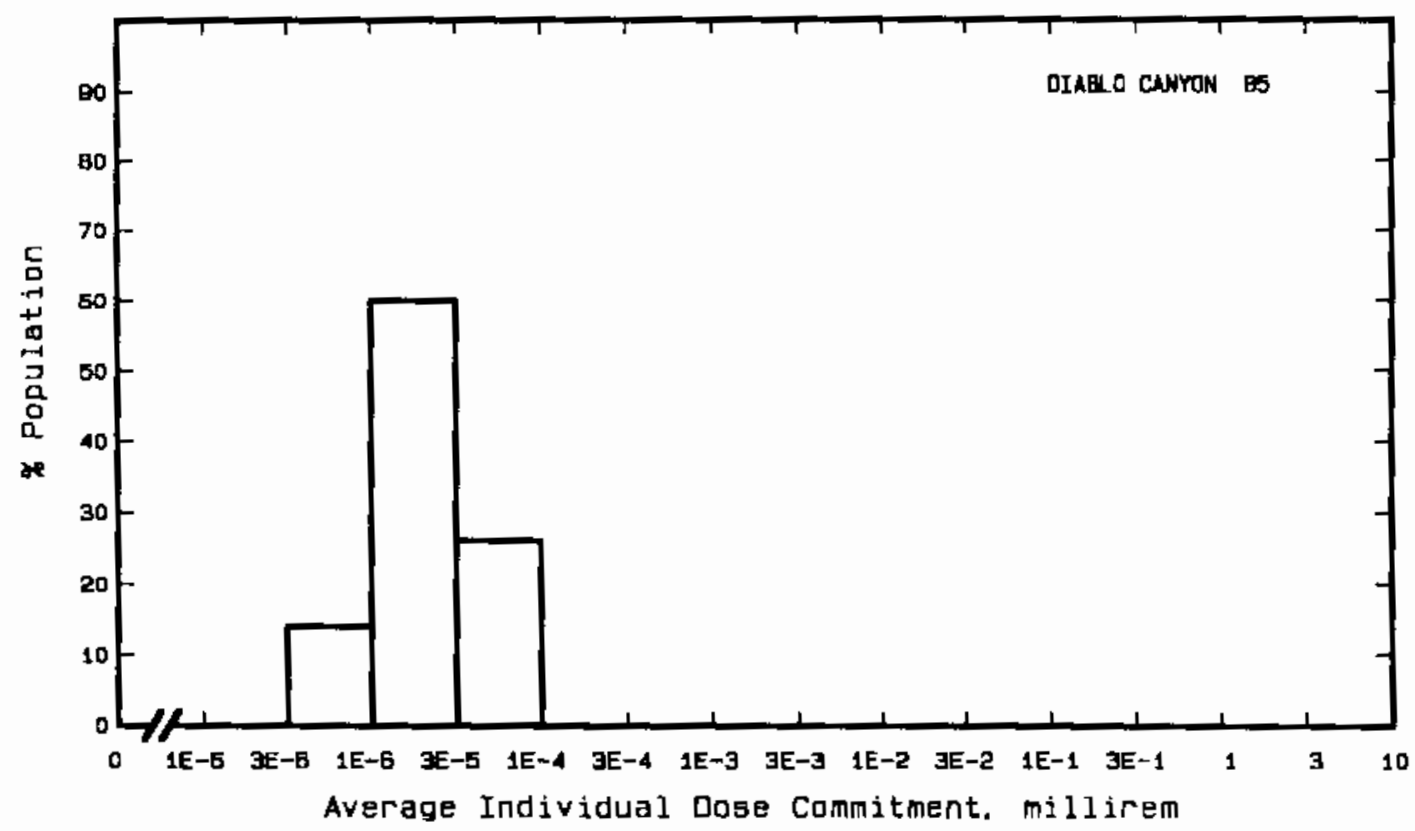


Site: DRESDEN

GRUNDY COUNTY, ILLINOIS

Location: $\quad$ N $41.3897^{\circ} \quad$ W $88.2711^{\circ}$

POPULATION DATA

Total Population Within 2-to-80-km Region: 6.4E6

Major Metropolitan Centers Within Region:

Center

Chicago SMSA

Gary-Hammond-E.Chicago-SMSA

Kankakee SMSA

Aurora

Joliet

Elgin

\section{Population}

$7,100,000$ (a)

$640,000(a)$

100,000

81,000

78,000

56,000
Location

$75 \mathrm{~km} \quad \mathrm{NE}$

$80 \mathrm{~km} \quad \mathrm{ENE}$

$45 \mathrm{~km} \quad \mathrm{SE}$

$41 \mathrm{~km} \mathrm{~N}$

$22 \mathrm{~km} \quad \mathrm{NE}$

$74 \mathrm{~km} \mathrm{~N}$

\section{SITE-SPECIFIC DATA - AIRBORNE PATHWAYS}

Average Annual State Production

of Crops and Animal Products

In $80-\mathrm{km}$ Radius Circle

Regional Productivity Factor:

Animal Grazing Factor:
Veg: $1.1 E 8$ kilogram

MiTk: 1.8E8 liter

Meat: $1.9 \mathrm{E} 8 \mathrm{kilogram}$

1

0.5

Meteorology Period of Record: 1 JAN 74 - 31 JAN 75 Recovery: $77 \%$

SITE-SPECIFIC DATA - WATERBORNE PATHWAYS viג ILLINOIS RIVER

Average River Flow

at Site: $12,000 \mathrm{ft}^{3} / \mathrm{s}$

Drinking Water:

Exposed Population:

Fish:

Edible Harvest: (b)

(a) Population of total SMSA given; population of SMSA fraction within $80 \mathrm{~km}$ of site would be somewhat smaller.

(b) River water used for sewage disposal for Chicago, so population doses from liquid pathways assumed to be near zero. 


\section{POPULATION DOSE-COMMITMENT ESTIMATES AND \\ AVERAGE INDIVIDUAL DOSE-COMMITMENT HISTOGRAM FOR}

DRESDEN 1,2 AND 3

Dose Commitments (person-rem) from Waterborne Pathways

Total Body GI-LLI Ihyroid $\underline{\text { Bone }}$

Infant

Child

Teen

Adult

(Little or No Waterborne Pathway Doses)

TOTAL

Dose Commitments (person-rem) from Airborne Pathways

Iotal Body GI-LLI Thyroid Bone Liver Lung

$\begin{array}{lllllll}\text { Infant } & 1.3 \mathrm{E}-02 & 1.3 \mathrm{E}-02 & 6.4 \mathrm{E}-02 & 1.3 \mathrm{E}-02 & 1.4 \mathrm{E}-02 & 1.5 \mathrm{E}-02 \\ \text { Child } & 1.5 \mathrm{E}-01 & 1.5 \mathrm{E}-01 & 6.2 \mathrm{E}-01 & 1.7 \mathrm{E}-01 & 1.5 \mathrm{E}-01 & 1.8 \mathrm{E}-01 \\ \text { Teen } & 1.1 \mathrm{E}-01 & 1.1 \mathrm{E}-01 & 3.4 \mathrm{E}-01 & 1.1 \mathrm{E}-01 & 1.1 \mathrm{E}-01 & 1.4 \mathrm{E}-01 \\ \text { Adu1t } & 6.8 \mathrm{E}-01 & 7.0 \mathrm{E}-01 & 1.6 \mathrm{E}+00 & 6.7 \mathrm{E}-01 & 6.8 \mathrm{E}-01 & 7.8 \mathrm{E}-01 \\ \text { TOTAL } & 9.6 \mathrm{E}-01 & 9.8 \mathrm{E}-01 & 2.7 \mathrm{E}+00 & 9.7 \mathrm{E}-01 & 9.6 \mathrm{E}-01 & 1.1 \mathrm{E}+00\end{array}$

Production/Consumption factors:

Produce: $0.091 \quad$ Milk: 0.21 Meat: 0.37

Fraction of Population Feceiving an Average Individual Total-Body Dose Commitment from Airborne Pathways

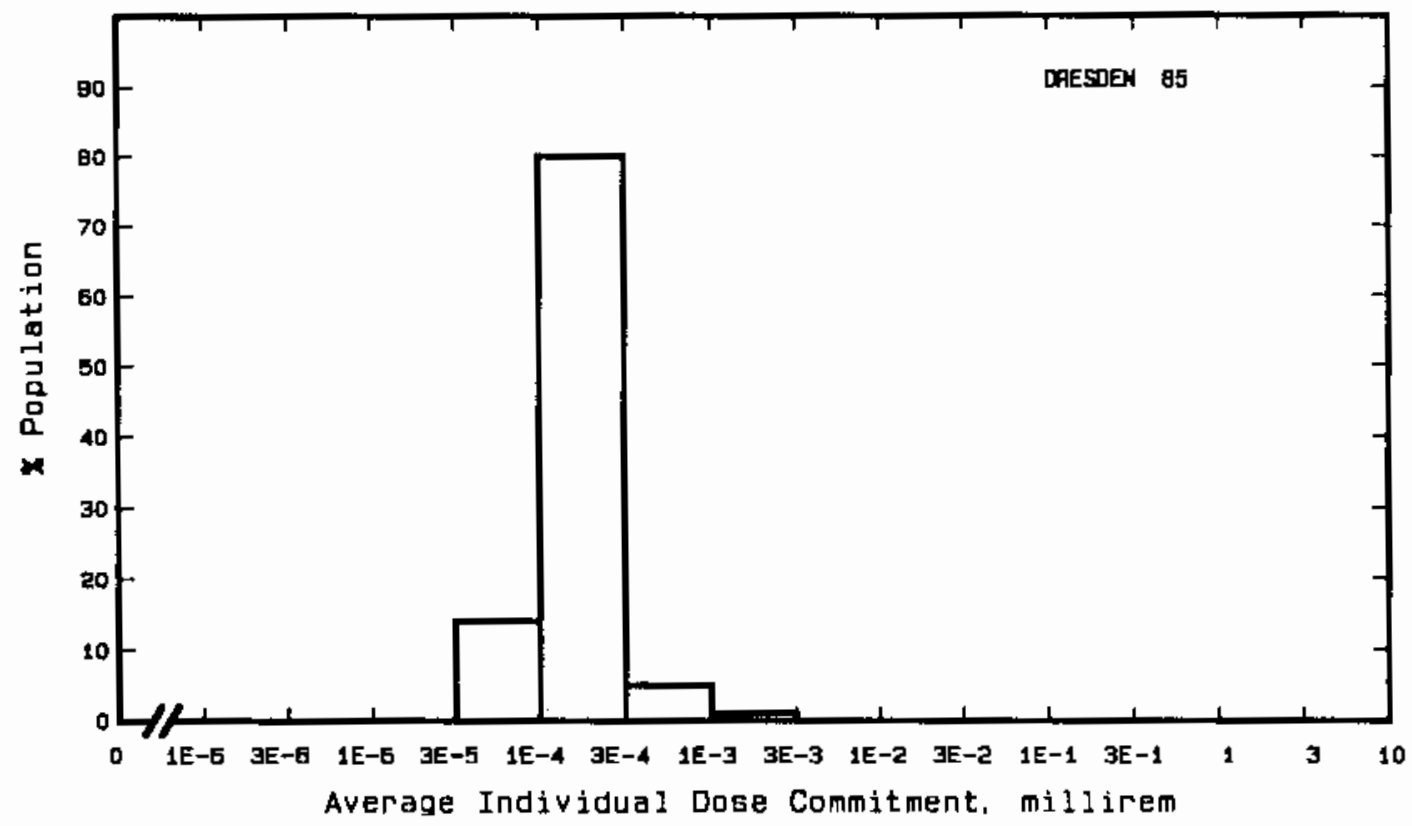


Site: DUANE ARNOLD

CEDAR RAPIDS, IOWA

Location: $\quad N \quad 42.1006^{\circ} \quad$ W $91.7772^{\circ}$

POPULATION DATA

Total Population Within 2-to-80-km Region: 6.0E5

Major Metropolitan Centers Within Region:

Center Population Location

$\begin{array}{llll}\text { Cedar Rapids SMSA } & 170,000 & 17 \mathrm{~km} & \text { SE }\end{array}$

Waterloo-Cedar Falls SMSA $140,000 \quad 64 \mathrm{~km}$ NW

Iowa City SMSA $\quad 82,000 \quad 52 \mathrm{~km} \quad$ SSE

Marion $19,000 \quad 16 \mathrm{~km}$ ESE

SITE-SPECIFIC DATA - AIRBORNE PATHWAYS

Average Annual State Production

of Crops and Animal Products

In $80-\mathrm{km}$ Radius Circle

Regional Productivity Factor:

Animal Grazing Factor:
Veg: $9.8 E 7$ kilogram

Milk: 2.6E8 liter

Meat: $4.2 \mathrm{~EB}$ kilogram

1

0.5

Meteorology Period of Record: 1 FEB $71-31$ DEC 75 Recovery: 57\%

SITE-SPECIFIC DATA - WATERBORNE PATHWAYS via CEDAR RIVER

Drinking Water:

Fish:
Average River Flow

at Site: $3,100 \mathrm{ft}^{3} / \mathrm{s}$

Exposed Population: $170,000^{(a)}$

Dilution Factor: 1

Edible Harvest: (b)

Dilution Factor: 1

(a) All persons in the Cedar Rapids SMSA assumed to drink river water.

(b) No fish catch data given in FES (1973); thus population was assumed to eat fish at generic consumption rates (Table A.1). 
POPULATION DOSE-COMMITMENT ESTIMATES AND
AVERAGE INDIVIDUAL DOSE-COMMITMENT HISTOGRAM FOR

DUANE ARNDLD

Dose Commitments (person-rem) from Waterborne Pathways

Iotal Body GI-LLI Thyroid Bone Liver

$\begin{array}{llllll}\text { Infant } & 4.6 \mathrm{E}-06 & 4.4 \mathrm{E}-06 & 1.6 \mathrm{E}-06 & 2.4 \mathrm{E}-06 & 2.9 \mathrm{E}-06 \\ \text { ChiTd } & 1.9 \mathrm{E}-04 & 1.7 \mathrm{E}-04 & 1.9 \mathrm{E}-05 & 1.2 \mathrm{E}-04 & 2.0 \mathrm{E}-04 \\ \text { Teen } & 1.2 \mathrm{E}-04 & 2.7 \mathrm{E}-04 & 7.4 \mathrm{E}-06 & 7.4 \mathrm{E}-05 & 1.7 \mathrm{E}-04 \\ \text { Adult } & 7.3 \mathrm{E}-04 & 2.4 \mathrm{E}-03 & 6.3 \mathrm{E}-05 & 5.1 \mathrm{E}-04 & 1.1 \mathrm{E}-03 \\ \text { TOTAL } & 1.0 \mathrm{E}-03 & 2.9 \mathrm{E}-03 & 9.1 \mathrm{E}-05 & 7.1 \mathrm{E}-04 & 1.4 \mathrm{E}-03\end{array}$

Dose Commitments (person-rem) from Airborne Pathways

Iotal Body GI-LLI Thyroid Bone Liver lung

$\begin{array}{lllllll}\text { Infant } & 3.0 \mathrm{E}-04 & 3.0 \mathrm{E}-04 & 5.6 \mathrm{E}-04 & 2.5 \mathrm{E}-04 & 3.0 \mathrm{E}-04 & 3.4 \mathrm{E}-04 \\ \text { Child } & 4.1 \mathrm{E}-03 & 4.1 \mathrm{E}-03 & 6.5 \mathrm{E}-03 & 2.8 \mathrm{E}-03 & 4.0 \mathrm{E}-03 & 4.6 \mathrm{E}-03 \\ \text { Teen } & 2.8 \mathrm{E}-03 & 2.9 \mathrm{E}-03 & 3.7 \mathrm{E}-03 & 2.0 \mathrm{E}-03 & 2.8 \mathrm{E}-03 & 3.3 \mathrm{E}-03 \\ \text { Adult } & 1.6 \mathrm{E}-02 & 1.7 \mathrm{E}-02 & 2.0 \mathrm{E}-02 & 1.2 \mathrm{E}-02 & 1.6 \mathrm{E}-02 & 1.8 \mathrm{E}-02 \\ \text { TOTAL } & 2.3 \mathrm{E}-02 & 2.5 \mathrm{E}-02 & 3.1 \mathrm{E}-02 & 1.7 \mathrm{E}-02 & 2.3 \mathrm{E}-02 & 2.7 \mathrm{E}-02\end{array}$

Production/Consumption factors:

Produce: 0.85 Milk: $3.3 \quad$ Meat: 8.8

Fraction of Population Receiving an Average Individual

Total-Body Dose Commitment from Airborne Pathways

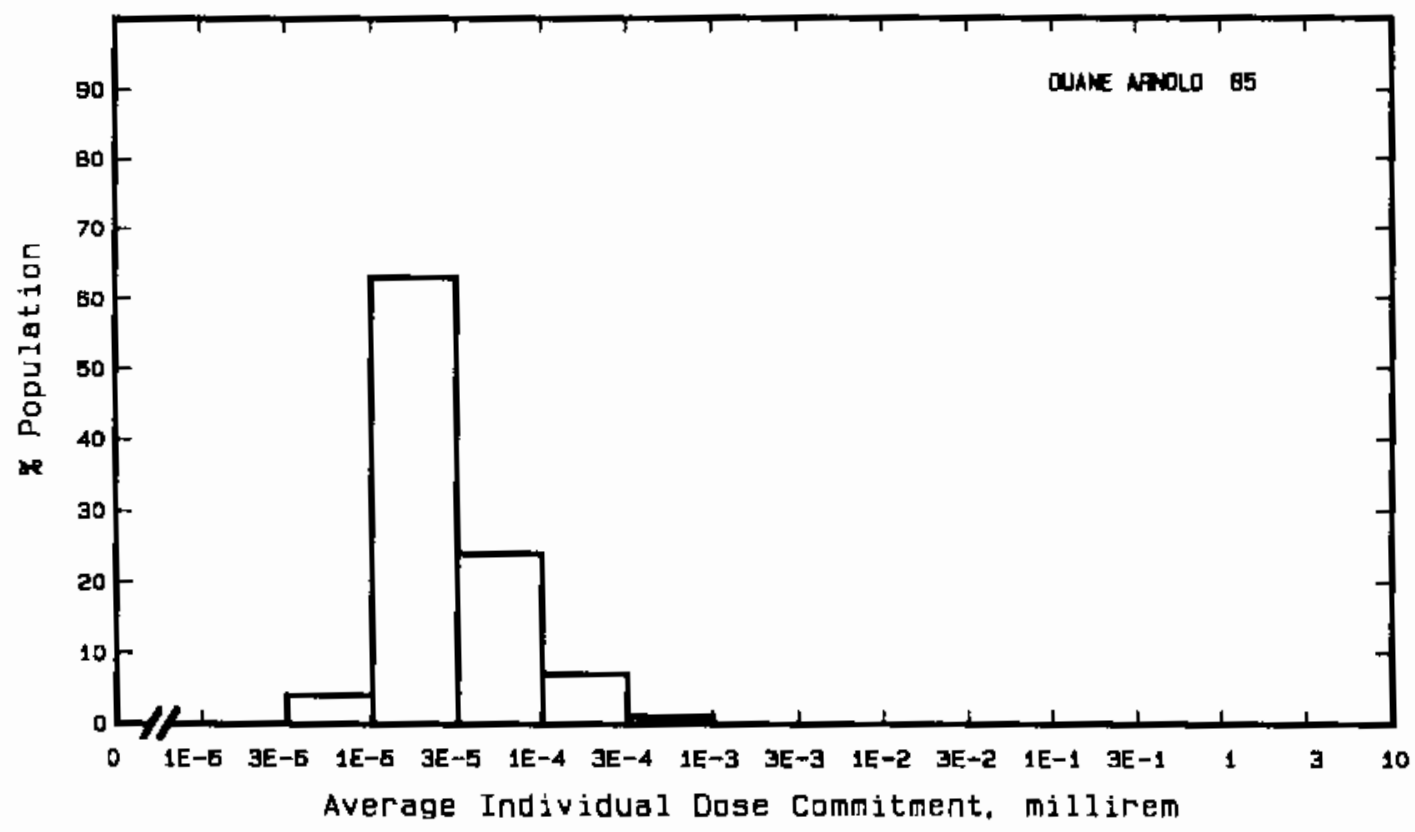


Site: J. M. FARLEY

DOTHAN, ALABAMA

Location: $\quad$ N $31.2228^{\circ} \quad$ W $85.1126^{\circ}$

POPULATION DATA

Total Population Within 2-to-80-km Region: 3.7E5

Major Metropolitan Centers Within Region:

Center

Dothan

Enterprise

0zark

Eufaula

Bainbridge
Population

50,000

18,000

13,000

12,000

11,000
Location

$27 \mathrm{~km} \quad \mathrm{~W}$

$71 \mathrm{~km} \quad W$

$56 \mathrm{~km} \quad W N W$

$75 \mathrm{~km} \mathrm{~N}$

$62 \mathrm{~km} \quad \mathrm{SE}$

SITE-SPECIFIC DATA - AIRBORNE PATHWAYS

Average Annual State Production

of Crops and Animal Products

In $80-\mathrm{km}$ Radius Circle

Regional Productivity Factor:

Animal Grazing Factor:

Meteorology Period of Record: 1 APR 71 - 31 MAR 75 Recovery: 100\%
Veg: $1.7 \mathrm{E} 7 \mathrm{ki} \log \mathrm{ram}$

Milk: 5.7 E7 liter

Meat: $8.6 E 7$ kilogram

0.95

0.8

SITE-SPECIFIC DATA - WATERBORNE PATHWAYS via CHATTAHOOCHEE RIVER

Average River Flow at Site: $12,000 \mathrm{ft}^{3} / \mathrm{s}$

Orinking Water:

Exposed Population: None

Fish:

Edible Harvest: $2.3 \mathrm{E} 5 \mathrm{~kg} / \mathrm{yr}$

Dilution Factor: 1 
POPULATION DOSE-COMMITMENT ESTIMATES AND

AVERAGE INDIVIDUAL DOSE-COMHITMENT HISTOGRAM FOR

J. M. FARLEY 1 AND 2

Dose Commitments (person-rem) from Waterborne Pathways

Total Body GI-LLI $\underline{\text { Thyroid }}$ Bone Liver

$\begin{array}{llllll}\text { Infant } & 0.0 \mathrm{E}+00 & 0.0 \mathrm{E}+00 & 0.0 \mathrm{E}+00 & 0.0 \mathrm{E}+00 & 0.0 \mathrm{E}+00 \\ \text { Child } & 5.6 \mathrm{E}-04 & 8.5 \mathrm{E}-04 & 2.7 \mathrm{E}-04 & 1.5 \mathrm{E}-03 & 1.9 \mathrm{E}-03 \\ \text { Teen } & 7.7 \mathrm{E}-04 & 1.5 \mathrm{E}-03 & 2.4 \mathrm{E}-04 & 8.8 \mathrm{E}-04 & 1.6 \mathrm{E}-03 \\ \text { Adu7t } & 7.6 \mathrm{E}-03 & 1.3 \mathrm{E}-02 & 1.9 \mathrm{E}-03 & 5.1 \mathrm{E}-03 & 1.0 \mathrm{E}-02 \\ \text { TOTAL } & 8.9 \mathrm{E}-03 & 1.5 \mathrm{E}-02 & 2.4 \mathrm{E}-03 & 7.4 \mathrm{E}-03 & 1.3 \mathrm{E}-02\end{array}$

Dose Commitments (person-rem) from Airborne Pathways

Iotal Body GI-LLI Thyroid Bone Liver Lung

$\begin{array}{lllllll}\text { Infant } & 1.7 \mathrm{E}-03 & 1.7 \mathrm{E}-03 & 3.3 \mathrm{E}-03 & 6.1 \mathrm{E}-04 & 1.7 \mathrm{E}-03 & 1.7 \mathrm{E}-03 \\ \text { Child } & 2.3 \mathrm{E}-02 & 2.3 \mathrm{E}-02 & 3.3 \mathrm{E}-02 & 6.8 \mathrm{E}-03 & 2.3 \mathrm{E}-02 & 2.3 \mathrm{E}-02 \\ \text { Teen } & 1.5 \mathrm{E}-02 & 1.5 \mathrm{E}-02 & 1.9 \mathrm{E}-02 & 5.0 \mathrm{E}-03 & 1.5 \mathrm{E}-02 & 1.6 \mathrm{E}-02 \\ \text { Adult } & \mathrm{B} .6 \mathrm{E}-02 & 8.6 \mathrm{E}-02 & 1.0 \mathrm{E}-01 & 3.0 \mathrm{E}-02 & 8.6 \mathrm{E}-02 & 8.8 \mathrm{E}-02 \\ \text { TOTAL } & 1.3 \mathrm{E}-01 & 1.3 \mathrm{E}-01 & 1.6 \mathrm{E}-01 & 4.2 \mathrm{E}-02 & 1.3 \mathrm{E}-01 & 1.3 \mathrm{E}-01\end{array}$

Production/Consumption factors:

Produce: $0.22 \quad$ Milk: 1.1 Meat: 2.8

Fraction of Population Receiving an Average Individual

Total-Body Dose Commitment from Airborne Pathways

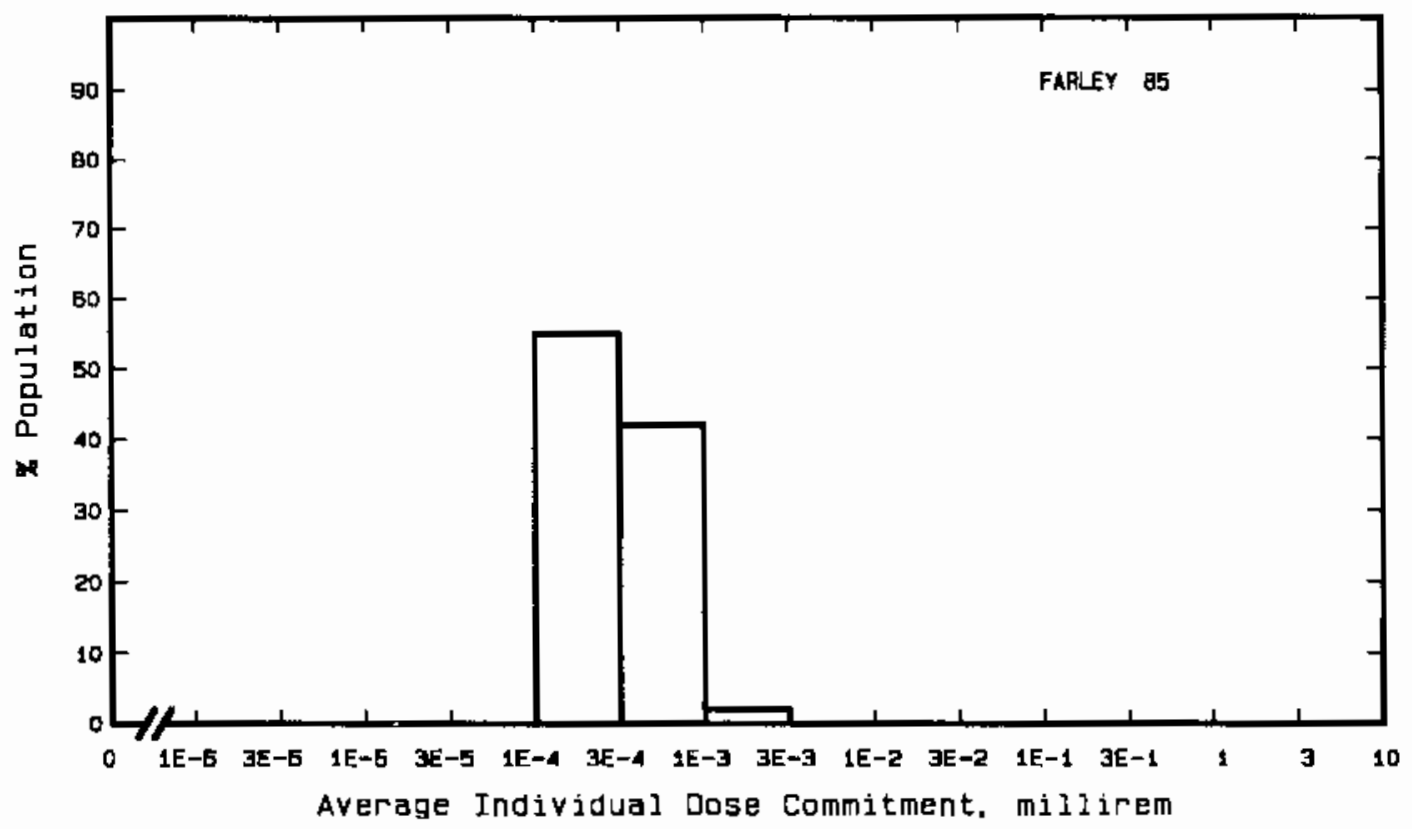


Site: J. A. FITZPATRICK

OSWEGO, NEW YORK

Location: N $43.5239^{\circ}$ W $76.3983^{\circ}$

POPULATION DATA

Total Population Within 2-to-80-km Region: 8.5E5

Major Metropolitan Centers Within Region:

Center

Syracuse SMSA

Rome

Auburn

Watertown

Kingston
Population

650,000

48,000

33,000

28,000

24,000
Location

$56 \mathrm{~km} \quad$ SSE

$80 \mathrm{~km} \quad \mathrm{ESE}$

$66 \mathrm{~km} \quad \mathrm{SSW}$

$64 \mathrm{~km} \quad \mathrm{NE}$

$79 \mathrm{~km} \mathrm{~N}$

SITE-SPECIFIC DATA - AIRBORNE PATHWAYS

Average Annual State Production

of Crops and Animal Products

In 80-km Radius Circle

Regional Productivity Factor:

Animal Grazing Factor:
Veg: $7.6 \mathrm{E} 7 \mathrm{ki}$ logram

Milk: 7.0E8 liter

Meat: $3.3 \mathrm{E} 7$ kilogram

0.7

0.5

Meteorology Period of Record: 1 JAN 74 - 31 DEC 75 Recovery: $97 \%$

SITE-SPECIFIC DATA - WATERBORNE PATHWAYS via LAKE ONTARIO

Average Dilution $\mathrm{Flow}$

from Plant: $690 \mathrm{ft}^{3} / \mathrm{s}$

Drinking Water:

Exposed Population: 540,000 (a)

Dilution Factor: $0.003(b)$

Fish:

Edible Harvest: $7.3 \mathrm{E} 5 \mathrm{~kg} / \mathrm{yr}$

(a) Population exposed to drinking water derived from Nine Mile Point FES (1974).

(b) Dilution factors derived from FES (1973). 
POPULATION DOSE-COMMITMENT ESTIMATES AND

AVERAGE INDIVIDUAL DOSE-COMMITMENT HISTOGRAM FOR

$$
\text { J. A. FITZPATRICK }
$$

Dose Commitments (person-rem) from Waterborne Pathways

Total Body GI-LLI $\underline{\text { Thyroid }}$ Bone

$\begin{array}{llllll}\text { Infant } & 3.4 \mathrm{E}-05 & 3.0 \mathrm{E}-05 & 1.0 \mathrm{E}-05 & 6.4 \mathrm{E}-05 & 1.1 \mathrm{E}-04 \\ \text { Child } & 1.5 \mathrm{E}-03 & 6.2 \mathrm{E}-04 & 1.1 \mathrm{E}-04 & 5.1 \mathrm{E}-03 & 6.4 \mathrm{E}-03 \\ \text { Teen } & 2.1 \mathrm{E}-03 & 7.1 \mathrm{E}-04 & 4.0 \mathrm{E}-05 & 2.8 \mathrm{E}-03 & 5.0 \mathrm{E}-03 \\ \text { Adult } & 2.2 \mathrm{E}-02 & 6.5 \mathrm{E}-03 & 3.3 \mathrm{E}-04 & 1.7 \mathrm{E}-02 & 3.0 \mathrm{E}-02 \\ \text { TOTAL } & 2.5 \mathrm{E}-02 & 7.9 \mathrm{E}-03 & 4.9 \mathrm{E}-04 & 2.5 \mathrm{E}-02 & 4.1 \mathrm{E}-02\end{array}$

Dose Commitments (person-rem) from Airborne Pathways

Total Body GI-LLI Ihyroid Bone Liver Lung

$\begin{array}{lllllll}\text { Infant } & 5.4 \mathrm{E}-03 & 5.4 \mathrm{E}-03 & 3.4 \mathrm{E}-02 & 5.6 \mathrm{E}-03 & 5.5 \mathrm{E}-03 & 5.5 \mathrm{E}-03 \\ \text { Child } & 6.1 \mathrm{E}-02 & 6.0 \mathrm{E}-02 & 2.5 \mathrm{E}-01 & 6.6 \mathrm{E}-02 & 6.1 \mathrm{E}-02 & 6.2 \mathrm{E}-02 \\ \text { Teen } & 4.4 \mathrm{E}-02 & 4.4 \mathrm{E}-02 & 1.2 \mathrm{E}-01 & 4.6 \mathrm{E}-02 & 4.4 \mathrm{E}-02 & 4.6 \mathrm{E}-02 \\ \text { Adult } & 2.7 \mathrm{E}-01 & 2.7 \mathrm{E}-01 & 5.1 \mathrm{E}-01 & 2.7 \mathrm{E}-01 & 2.7 \mathrm{E}-01 & 2.7 \mathrm{E}-01 \\ \text { TOTAL } & 3.8 \mathrm{E}-01 & 3.8 \mathrm{E}-01 & 9.1 \mathrm{E}-01 & 3.9 \mathrm{E}-01 & 3.8 \mathrm{E}-01 & 3.9 \mathrm{E}-01\end{array}$

Production/Consumption factors:

Produce: 0.32

Milk: 4.4

Meat: 0.34

Fraction of Population Receiving an Average Individual

Total-Bady Dose Commitment from Airborne Pathways

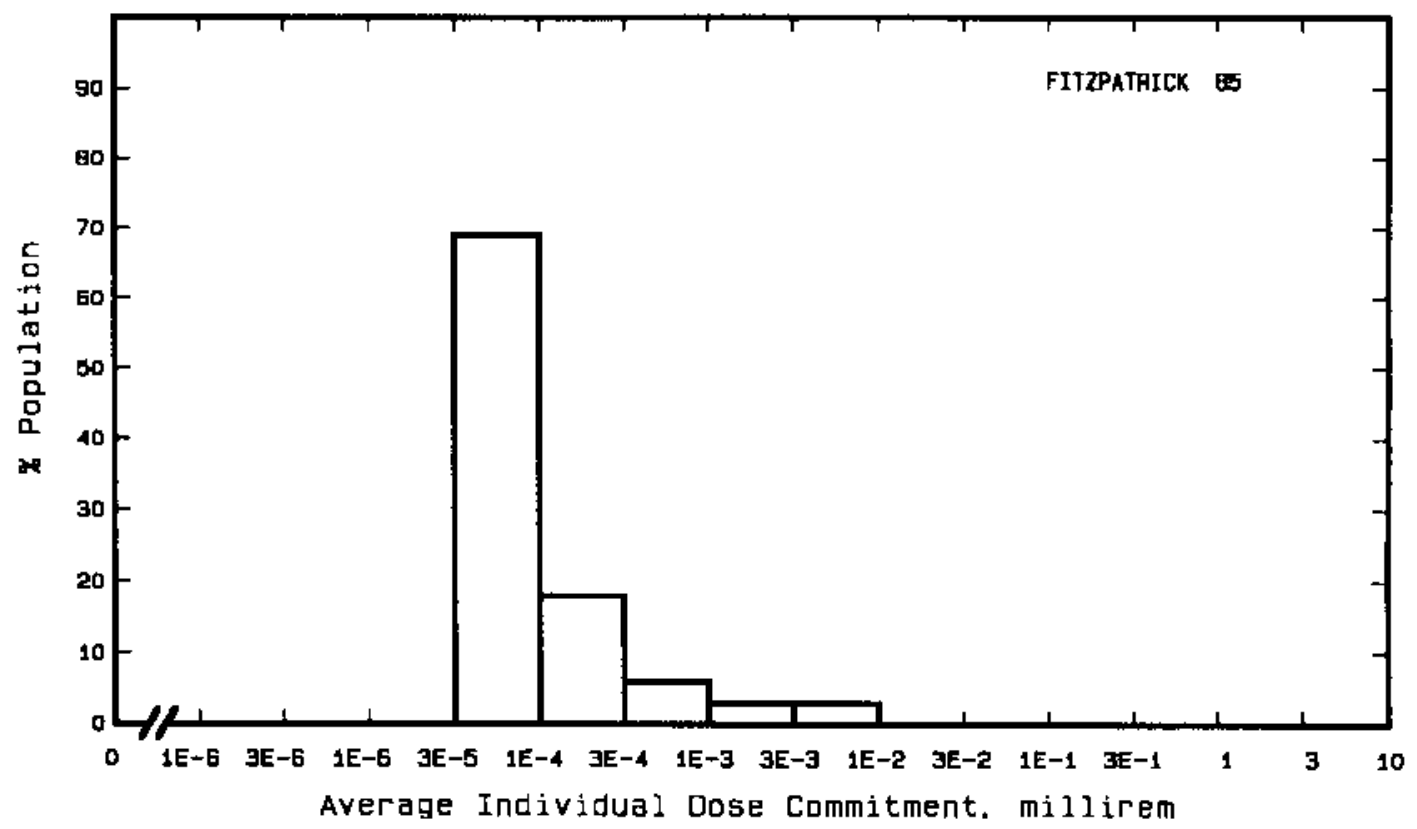


Site: FORT CALHOUN

WASHINGTON COUNTY, NEBRASKA

Location: $\quad N 41.5208^{\circ} \quad W 96.0767^{\circ}$

POPULATION DATA

Total Population Within 2-to-80-km Region: 7.6E5

Major Metropolitan Centers Within Region:

\begin{tabular}{lrrrl}
\multicolumn{1}{c}{ Center } & Population & \multicolumn{3}{c}{ Location } \\
Omaha SMSA & 610,000 & & & \\
Council Bluffs & 56,000 & $32 \mathrm{~km}$ & SSE \\
Freemont & 24,000 & $34 \mathrm{~km}$ & SE \\
Bellevue & 22,000 & $36 \mathrm{~km}$ & WSW \\
& & $44 \mathrm{~km}$ & SSE
\end{tabular}

SITE-SPECIFIC DATA - AIRBORNE PATHWAYS

Average Annual State Production

of Crops and Animal Products

In $80-\mathrm{km}$ Radius Circle

Regional Productivity Factor:

Animal Grazing Factor:
Veg: $9.7 E 7$ kilogram

Milk: 7.2E7 liter

Meat: 2.0E8 kilogram

1

0.5

Meteorology Period of Record: 1 JAN 74 - 31 DEC 74 Recovery: 98\%

SITE-SPECIFIC DATA - WATERBORNE PATHWAYS via MISSOURI RIVER

Average River Flow

at Site: $27,000 \mathrm{ft}^{3} / \mathrm{s}$

Drinking Water:

Exposed Population: $580,000^{(a)}$

Dilution Factor: 1

Fish:

Edible Harvest: $1.0 \mathrm{E} 4 \mathrm{~kg} / \mathrm{yr}$

Dilution Factor: 1

(a) Drinking water population assumed to be approximately Omaha SMSA (FES 1972). 


\section{POPULATION DOSE-COMMITMENT ESTIMATES AND \\ AVERAGE INDIVIDUAL DOSE-COMMITMENT HISTOGRAM FOR \\ FORT CALHOUN}

Dose Commitments (person-rem) from Waterborne Pathways

Total Body GI-LLI Ihyroid Bone Liver

$\begin{array}{llllll}\text { Infant } & 3.4 \mathrm{E}-03 & 3.2 \mathrm{E}-03 & 1.0 \mathrm{E}-02 & 2.6 \mathrm{E}-03 & 6.2 \mathrm{E}-03 \\ \text { Child } & 4.1 \mathrm{E}-02 & 3.7 \mathrm{E}-02 & 8.5 \mathrm{E}-02 & 2.9 \mathrm{E}-02 & 6.3 \mathrm{E}-02 \\ \text { Teen } & 1.8 \mathrm{E}-02 & 1.5 \mathrm{E}-02 & 2.8 \mathrm{E}-02 & 7.9 \mathrm{E}-03 & 2.4 \mathrm{E}-02 \\ \text { Adu7t } & 1.6 \mathrm{E}-01 & 1.3 \mathrm{E}-01 & 2.2 \mathrm{E}-01 & 5.0 \mathrm{E}-02 & 1 . \mathrm{BE}-01 \\ \text { TOTAL } & 2.3 \mathrm{E}-01 & 1.9 \mathrm{E}-01 & 3.4 \mathrm{E}-01 & 9.0 \mathrm{E}-02 & 2.8 \mathrm{E}-01\end{array}$

Dose Commitments (person-rem) from Airborne Pathways

Iotal Body GI-LLI $\underline{\text { Thyroid }}$ Bone Liver Lung

$\begin{array}{lllllll}\text { Infant } & 2.1 \mathrm{E}-04 & 2.0 \mathrm{E}-04 & 2.8 \mathrm{E}-03 & 2.1 \mathrm{E}-04 & 2.2 \mathrm{E}-04 & 2.2 \mathrm{E}-04 \\ \text { Child } & 2.3 \mathrm{E}-03 & 2.3 \mathrm{E}-03 & 2.8 \mathrm{E}-02 & 2.4 \mathrm{E}-03 & 2.4 \mathrm{E}-03 & 2.5 \mathrm{E}-03 \\ \text { Teen } & 1.7 \mathrm{E}-03 & 1.7 \mathrm{E}-03 & 1.1 \mathrm{E}-02 & 1.7 \mathrm{E}-03 & 1.7 \mathrm{E}-03 & 2.0 \mathrm{E}-03 \\ \text { Adult } & 1.0 \mathrm{E}-02 & 1.0 \mathrm{E}-02 & 4.5 \mathrm{E}-02 & 1.0 \mathrm{E}-02 & 1.0 \mathrm{E}-02 & 1.1 \mathrm{E}-02 \\ \text { TOTAL } & 1.4 \mathrm{E}-02 & 1.4 \mathrm{E}-02 & 8.7 \mathrm{E}-02 & 1.4 \mathrm{E}-02 & 1.5 \mathrm{E}-02 & 1.6 \mathrm{E}-02\end{array}$

Production/Consumption factors:

Produce: 0.65

Milk: $\quad 0.72$

Meat: 3.2

Fraction of Population Receiving an Average Individual Total-Bady Dase Commitment from Airborne Pathways

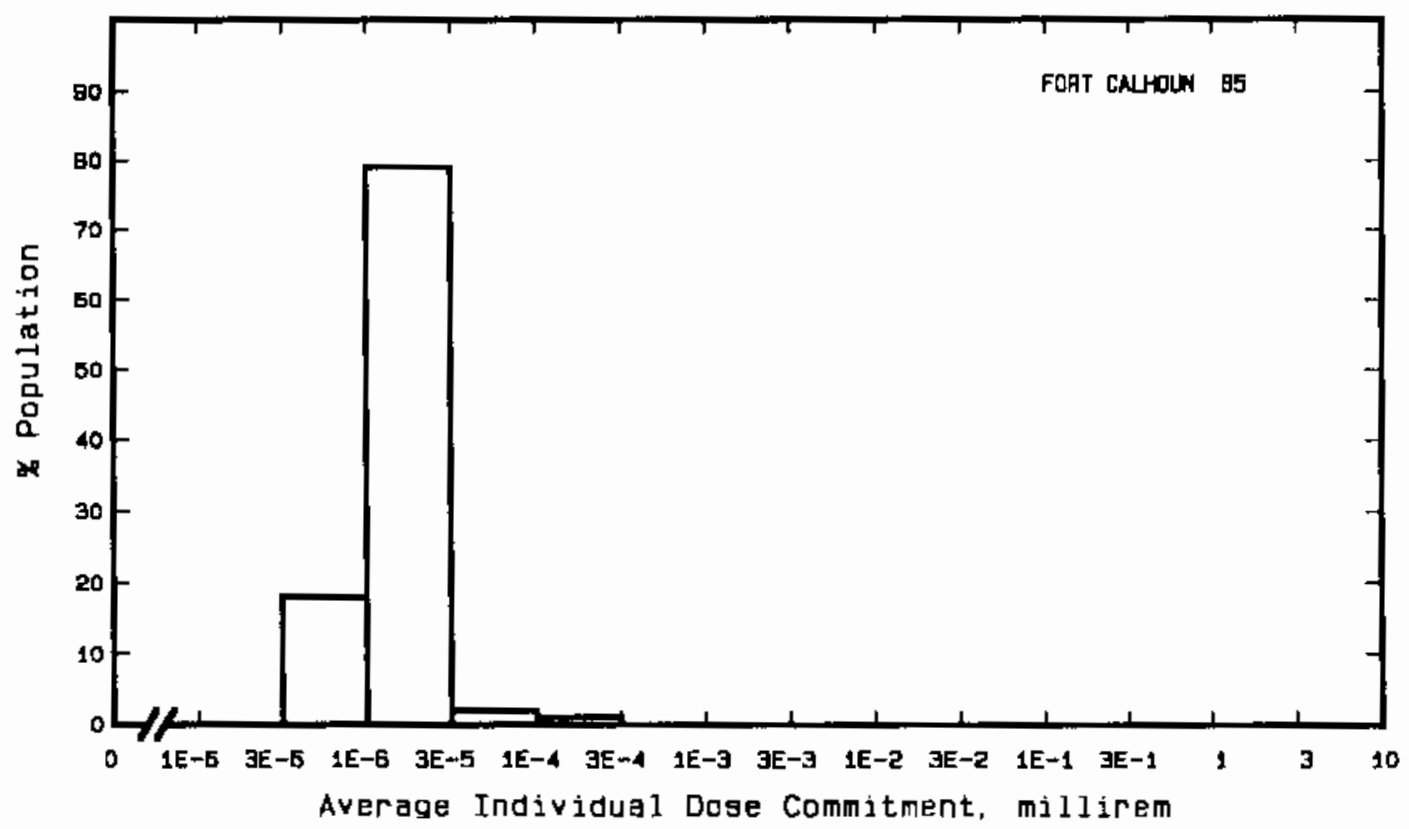


Site: R. E. GINNA ONTARIO, NEW YORK

Location: $\quad \mathrm{N} 43.2778^{\circ} \quad$ W $77.3089^{\circ}$

POPULATION DATA

Total Poputation Within 2-to-80-km Region: 1.2E6

Major Metropolitan Centers Within Region:

\begin{tabular}{lrrrr}
\multicolumn{1}{c}{ Center } & Population & & \multicolumn{2}{c}{ Location } \\
\cline { 2 - 4 } & & & \\
Rochester SMSA & 980,000 & $27 \mathrm{~km}$ & WSW \\
Auburn & 32,000 & $71 \mathrm{~km}$ & ESE \\
Oswego & 20,000 & $67 \mathrm{~km}$ & ENE \\
Batavia & 17,000 & $78 \mathrm{~km}$ & WSW \\
Geneva & 15,000 & $52 \mathrm{~km}$ & SSE
\end{tabular}

SITE-SPECIFIC DATA - AIRBORNE PATHWAYS

Average Annual State Production

of Crops and Animal Products

In BO-km Radius Circle

Regional Productivity Factor:

Animal Grazing Factor:
Veg: $7.6 \mathrm{E} 7 \mathrm{kilogram}$

Milk: 7.0EB liter

Meat: $3.3 \mathrm{E} 7 \mathrm{ki}$ logram

0.6

0.5

Meteorology Period of Record: 1 JAN 66 - 31 DEC 67 Recovery: 89\%

SITE-SPECIFIC DATA - WATERBORNE PATHWAYS via LAKE ONTARIO

Average Dilution Flow

from Plant: $770 \mathrm{ft}^{3} / \mathrm{s}$

Drinking Water:

Exposed Population: 560,000

Dilution Factor: $0.01^{(a)}$

Fish:

Edible Harvest: $7.3 \mathrm{E} 5 \mathrm{~kg} / \mathrm{yr}$

Dilution Factor: $0.01^{(a)}$

(a) Dilution factors from fES (1973). 
POPULATION DOSE-COMMITMENT ESTIMATES AND

AVERAGE INDIVIDUAL DOSE-COMMITMENT HISTOGRAM FOR

\author{
R. E. GINNA
}

Dose Commitments (person-rem) from Waterborne Pathways

Tota1 Body GI-LLI Ihyroid Bone Liver

$\begin{array}{llllll}\text { Infant } & 3.3 \mathrm{E}-03 & 3.2 \mathrm{E}-03 & 3.5 \mathrm{E}-03 & 5.1 \mathrm{E}-03 & 3.9 \mathrm{E}-03 \\ \text { Child } & 4.2 \mathrm{E}-02 & 3.7 \mathrm{E}-02 & 3.7 \mathrm{E}-02 & 7.1 \mathrm{E}-02 & 6.6 \mathrm{E}-02 \\ \text { Teen } & 2.3 \mathrm{E}-02 & 1.5 \mathrm{E}-02 & 1.4 \mathrm{E}-02 & 2.6 \mathrm{E}-02 & 3.6 \mathrm{E}-02 \\ \text { Adult } & 2.2 \mathrm{E}-01 & 1.3 \mathrm{E}-01 & 1.2 \mathrm{E}-01 & 1.5 \mathrm{E}-01 & 2.5 \mathrm{E}-01 \\ \text { TOTAL } & 2.8 \mathrm{E}-01 & 1.8 \mathrm{E}-01 & 1.7 \mathrm{E}-01 & 2.6 \mathrm{E}-01 & 3.6 \mathrm{E}-01\end{array}$

Dose Commitments (person-rem) from Airborne Pathways

Total Body GI-LLI Thyroid Bone Liver Lung

$\begin{array}{lllllll}\text { Infant } & 5.1 \mathrm{E}-04 & 5.1 \mathrm{E}-04 & 1.1 \mathrm{E}-03 & 1.8 \mathrm{E}-04 & 5.1 \mathrm{E}-04 & 5.2 \mathrm{E}-04 \\ \text { Child } & 6.5 \mathrm{E}-03 & 6.5 \mathrm{E}-03 & 9.8 \mathrm{E}-03 & 1.9 \mathrm{E}-03 & 6.5 \mathrm{E}-03 & 6.6 \mathrm{E}-03 \\ \text { Teen } & 4.2 \mathrm{E}-03 & 4.2 \mathrm{E}-03 & 5.5 \mathrm{E}-03 & 1.4 \mathrm{E}-03 & 4.2 \mathrm{E}-03 & 4.4 \mathrm{E}-03 \\ \text { Adu7t } & 2.3 \mathrm{E}-02 & 2.3 \mathrm{E}-02 & 2.7 \mathrm{E}-02 & 8.6 \mathrm{E}-03 & 2.3 \mathrm{E}-02 & 2.4 \mathrm{E}-02 \\ \text { TOTAL } & 3.4 \mathrm{E}-02 & 3.4 \mathrm{E}-02 & 4.4 \mathrm{E}-02 & 1.2 \mathrm{E}-02 & 3.4 \mathrm{E}-02 & 3.5 \mathrm{E}-02\end{array}$

Production/Consumption factors:

Produce: 0.20

Milk: 2.7

Meat: 0.20

Fraction of Population Receiving an Average Individual

Total-Body Dose Commitment from Airtorne Pathways

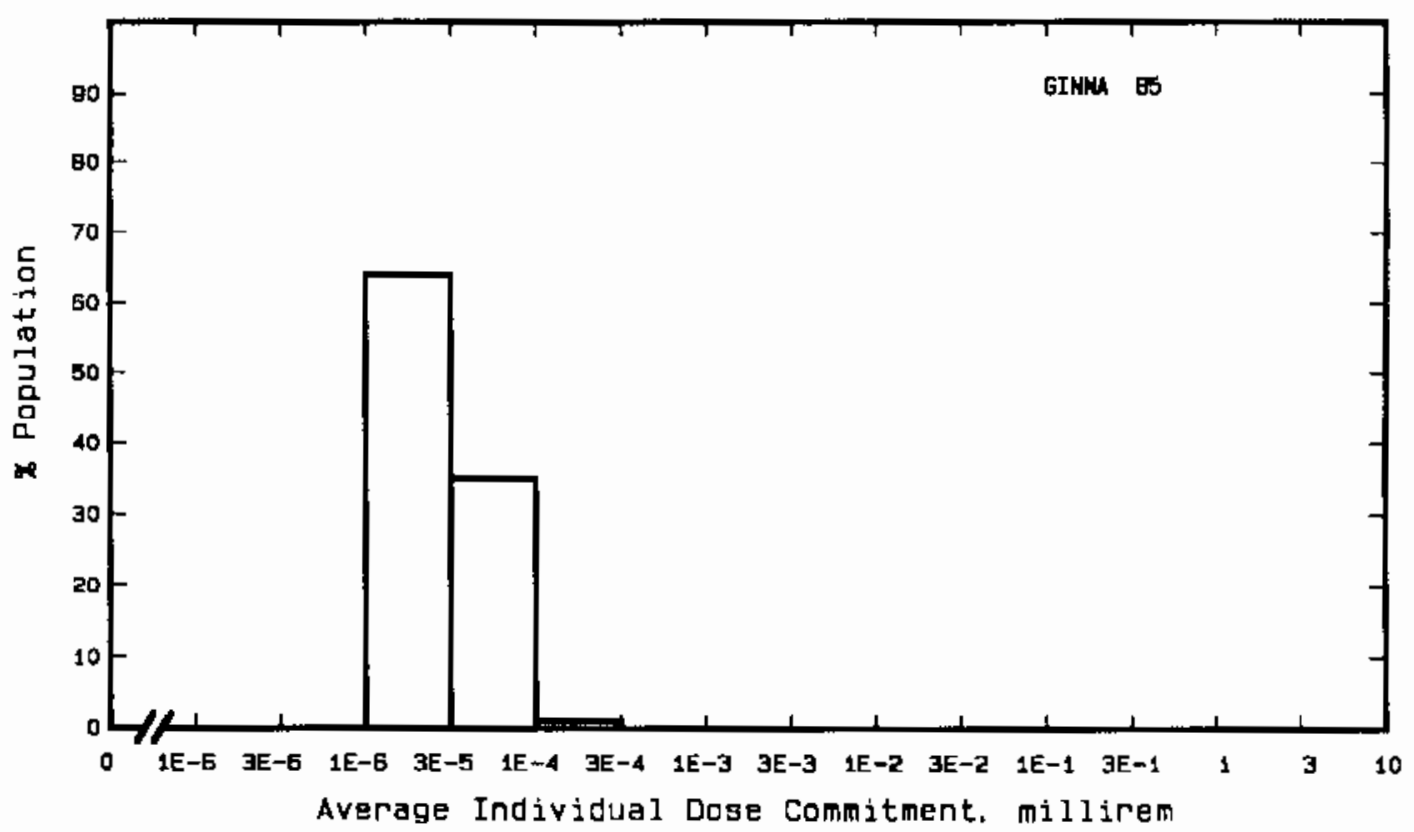


Site: GRAND GULF

PORT GIESON, MISSISSIPPI

Location: $\quad N 32.0270^{\circ} \quad$ W $91.2530^{\circ}$

POPULATION DATA

Total Population Within 2-to-80-km Region: 3.3E6

Major Metropolitan Centers Within Region:

Center

Vicksburg

Tallulah

Natches

Brookhaven
Population

27,000

10,000

20,000

11,000
Location

$40 \mathrm{~km} \quad$ NNE

$45 \mathrm{~km} \quad \mathrm{NNW}$

$60 \mathrm{~km} \quad \mathrm{SSW}$

$76 \mathrm{~km} \quad \mathrm{SE}$

SITE-SPECIFIC DATA - AIRBORNE PATHWAYS

Average Annual State Production

of Crops and Animal Products

In 80-km Radius Circle

Regional Productivity Factor:

Animal Grazing Factor:
Veg: 4.4E6 kilogram

Mi]k: $7.1 E 7$ liter

Meat: $9.9 E 7$ kilogram

0.9

0.8

Meteorology Period of Record: 1 AUG 72 - 31 JUL 73 Recovery: $99 \%$

SITE-SPECIFIC DATA - WATERBORNE PATHWAYS via MISSISSIPPI RIVER

Average River

Flow at Site: $430,000 \mathrm{ft}^{3} / \mathrm{s}$

Drinking Water: Exposed Population: None

Fish:

Edible Harvest: $7.0 \mathrm{E} 5 \mathrm{~kg} / \mathrm{yr}$

Dilution Factor: 1

Invertebrates:

Edible Harvest: (a)

Dilution Factor:

(a) Freshwater shellfish not considered. 
POPULATION DOSE-COMMITMENT ESTIMATES AND

AVERAGE INDIVIDUAL DOSE-COMMITMENT HISTOGRAM FOR

GRAND GULF

Dose Commitments (person-rem) from Waterborne Pathways

Total Body GI-LLI Ihyroid Bone Liver

$\begin{array}{llllll}\text { Infant } & 0.0 \mathrm{E}+00 & 0.0 \mathrm{E}+00 & 0.0 \mathrm{E}+00 & 0.0 \mathrm{E}+00 & 0.0 \mathrm{E}+00 \\ \text { Child } & 2.1 \mathrm{E}-05 & 3.0 \mathrm{E}-05 & 3.6 \mathrm{E}-07 & 2.5 \mathrm{E}-05 & 4.1 \mathrm{E}-05 \\ \text { Teen } & 1.5 \mathrm{E}-05 & 6.9 \mathrm{E}-05 & 2.8 \mathrm{E}-07 & 1.9 \mathrm{E}-05 & 3.9 \mathrm{E}-05 \\ \text { Adult } & 9.7 \mathrm{E}-05 & 6.4 \mathrm{E}-04 & 1.9 \mathrm{E}-06 & 1.4 \mathrm{E}-04 & 2.4 \mathrm{E}-04 \\ \text { TOTAL } & 1.3 \mathrm{E}-04 & 7.3 \mathrm{E}-04 & 2.6 \mathrm{E}-06 & 1.8 \mathrm{E}-04 & 3.2 \mathrm{E}-04\end{array}$

Dose Commitments (person-rem) from Airborne Pathways

Total Body GI-LLI Thyroid Bone Liver Lung

$\begin{array}{lllllll}\text { Infant } & 2.1 \mathrm{E}-05 & 2.1 \mathrm{E}-05 & 8.4 \mathrm{E}-05 & 1.8 \mathrm{E}-05 & 2.1 \mathrm{E}-05 & 2.5 \mathrm{E}-05 \\ \text { Child } & 2.5 \mathrm{E}-04 & 4.5 \mathrm{E}-04 & 5.8 \mathrm{E}-04 & 2.1 \mathrm{E}-04 & 2.5 \mathrm{E}-04 & 2.9 \mathrm{E}-04 \\ \text { Teen } & 1.8 \mathrm{E}-04 & 4.2 \mathrm{E}-04 & 3.1 \mathrm{E}-04 & 1.5 \mathrm{E}-04 & 1.7 \mathrm{E}-04 & 2.2 \mathrm{E}-04 \\ \text { Adult } & 1.0 \mathrm{E}-03 & 3.3 \mathrm{E}-03 & 1.5 \mathrm{E}-03 & 9.1 \mathrm{E}-04 & 1.0 \mathrm{E}-03 & 1.2 \mathrm{E}-03 \\ \text { TOTAL } & 1.5 \mathrm{E}-03 & 4.2 \mathrm{E}-03 & 2.5 \mathrm{E}-03 & 1.3 \mathrm{E}-03 & 1.5 \mathrm{E}-03 & 1.7 \mathrm{E}-03\end{array}$

Production/Consumption factors:

Produce: 0.060 Milk: $1.5 \quad$ Meat: 3.3

Fraction of Population Receiving an Average Individual

Total-Body Dose Commitment from Airborne Patnways

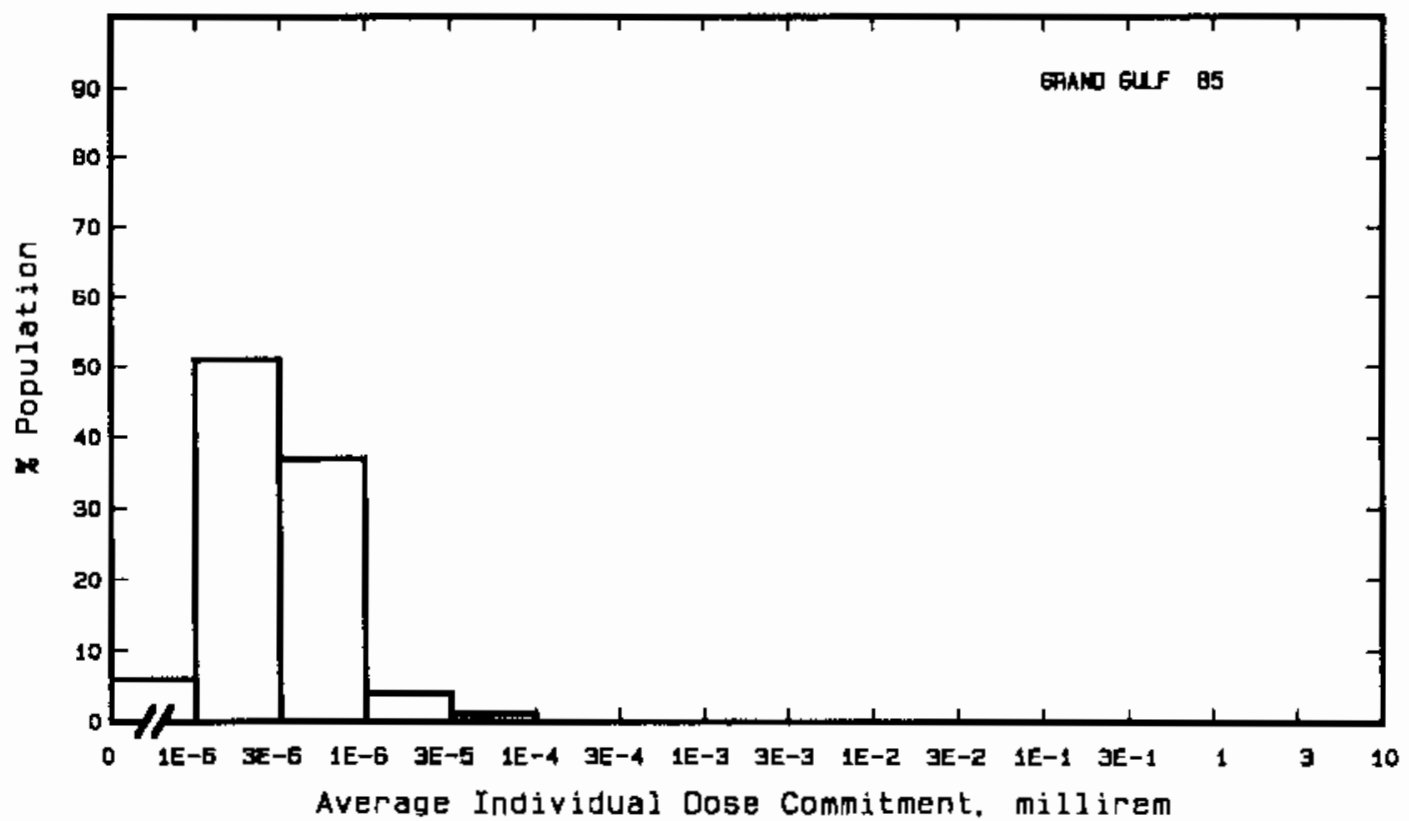


Site: HADDAM NECK

HADDAM NECK, CONNECTICUT

Location: $\quad$ N $41.4819^{\circ}$ W $72.4992^{\circ}$

POPULATION DATA

Total Population Within 2-to-80-km Region: 3.5E6

Major Metropolitan Centers Within Region:

Center

Hartford SHSA

Springfield-Chicopee-Holyoke SMSA

New Haven-West Haven SMSA

Bridgeport SMSA

New London-Norwich
Population

740,000

540,000

430,000

410,000

250,000
Location

$35 \mathrm{~km} \quad \mathrm{NNW}$

$70 \mathrm{~km} \quad \mathrm{~N}$

$40 \mathrm{~km} \quad W S W$

$66 \mathrm{~km} \quad$ WSW

$35 \mathrm{~km} \quad \mathrm{ESE}$

SITE-SPECIFIC DATA - AIRBORNE PATHWAYS

Average Annual State Production

of Crops and Animal Products

In $80-\mathrm{km}$ Radius Circle

Regional Productivity Factor:

Animal Grazing Factor:

Meteorology Period of Record: 1 JAN 75 - 31 DEC 75 Recovery: 95\%
Veg: $3.2 \mathrm{E} 7$ kilogram

Milk: 4.4E8 liter

Meat: $2.0 \mathrm{E} 7 \mathrm{kilogram}$

0.7

0.6

SITE-SPECIFIC DATA - WATERBORNE PATHWAYS via DISCHARGE CANAL TO CONN.

RIVER

Average Discharge Canal

Flow at Site: $840 \mathrm{ft}^{3} / \mathrm{s}$

Drinking Water:

Exposed Poputation: None

Fish:

Edible Harvest: $9.13^{(a)} \mathrm{kg} / \mathrm{yr}$

Dilution Factor: 1

(a) Caught in discharge canal according to FES (1973). 
POPULATION DOSE-COMMITMENT ESTIMATES AND

AVERAGE INDIVIDUAL DOSE-COMMITMENT HISTOGRAM FOR

HADDAM NECK

Dose Commitments (person-rem) from Waterborne Pathways

Total Body GI-LLI $\underline{\text { Thyroid }}$ Bone Liver

$\begin{array}{llllll}\text { Infant } & 0.0 \mathrm{E}+00 & 0.0 \mathrm{E}+00 & 0.0 \mathrm{E}+00 & 0.0 \mathrm{E}+00 & 0.0 \mathrm{E}+00 \\ \text { Child } & 2.8 \mathrm{E}-03 & 8.8 \mathrm{E}-04 & 1.2 \mathrm{E}-03 & 1.0 \mathrm{E}-02 & 1.2 \mathrm{E}-02 \\ \text { Teen } & 4.7 \mathrm{E}-03 & 9.2 \mathrm{E}-04 & 1.0 \mathrm{E}-03 & 6.1 \mathrm{E}-03 & 1.1 \mathrm{E}-02 \\ \text { Adult } & 4.8 \mathrm{E}-02 & 7.5 \mathrm{E}-03 & 7.5 \mathrm{E}-03 & 3.6 \mathrm{E}-02 & 6.4 \mathrm{E}-02 \\ \text { TOTAL } & 5.6 \mathrm{E}-02 & 9.3 \mathrm{E}-03 & 9.7 \mathrm{E}-03 & 5.2 \mathrm{E}-02 & 8.7 \mathrm{E}-02\end{array}$

Dose Commitments (person-rem) from Airborne Pathways

Total Body GI-LLI Thyroid bone liver

$\begin{array}{lllllll}\text { Infant } & 7.8 \mathrm{E}-03 & 7.8 \mathrm{E}-03 & 9.1 \mathrm{E}-03 & 5.7 \mathrm{E}-03 & 7.8 \mathrm{E}-03 & 8.2 \mathrm{E}-03 \\ \text { Child } & 9.1 \mathrm{E}-02 & 9.1 \mathrm{E}-02 & 9.8 \mathrm{E}-02 & 6.3 \mathrm{E}-02 & 9.1 \mathrm{E}-02 & 9.7 \mathrm{E}-02 \\ \text { Teen } & 6.5 \mathrm{E}-02 & 6.5 \mathrm{E}-02 & 6.8 \mathrm{E}-02 & 4.6 \mathrm{E}-02 & 6.5 \mathrm{E}-02 & 7.3 \mathrm{E}-02 \\ \text { Adult } & 3.8 \mathrm{E}-01 & 3.8 \mathrm{E}-01 & 3.9 \mathrm{E}-01 & 2.8 \mathrm{E}-01 & 3.8 \mathrm{E}-01 & 4.1 \mathrm{E}-01 \\ \text { TOTAL } & 5.5 \mathrm{E}-01 & 5.5 \mathrm{E}-01 & 5.7 \mathrm{E}-01 & 3.9 \mathrm{E}-01 & 5.5 \mathrm{E}-01 & 5.9 \mathrm{E}-01\end{array}$

Production/Consumption factors:

Produce: 0.033 Milk: 0.68 Meat: 0.052

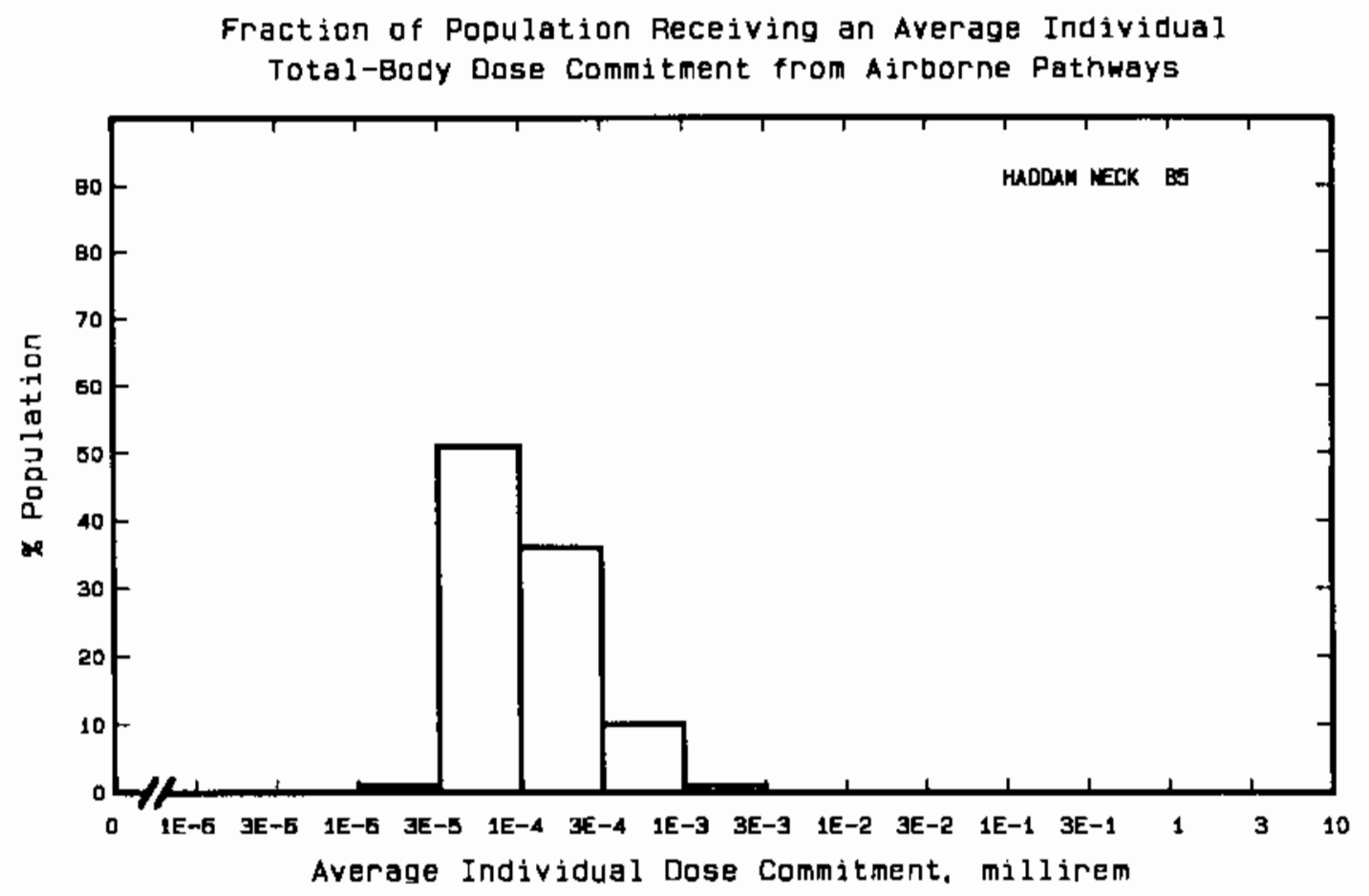


Site: E. I. HATCH

BAXLEY, GEORGIA

Location: N $31.9342^{\circ} \quad$ W $82.3444^{\circ}$

POPULATION DATA

Total Population Within 2-to-80-km Region: 3.3E5

Major Metropolitan Centers Within Region:

\begin{tabular}{lccl} 
Center & Population & \multicolumn{2}{l}{ Location } \\
Waycross & 21,000 & & \\
Statesboro & 16,000 & $80 \mathrm{~km}$ & $\mathrm{~S}$ \\
Hinesville & 12,000 & $78 \mathrm{~km}$ & $\mathrm{NE}$ \\
Douglas & 12,000 & $71 \mathrm{~km}$ & E \\
Vidalia & 10,000 & $67 \mathrm{~km}$ & $\mathrm{SW}$ \\
& & $32 \mathrm{~km}$ & $\mathrm{~N}$
\end{tabular}

SITE-SPECIFIC DATA - AIRBORNE PATHWAYS

Average Annual State Production

of Crops and Animal Products

In $80-\mathrm{km}$ Radius Circle

Regional Productivity Factor:

Animal Grazing Factor:
Veg: $8.8 \mathrm{E} 6$ kilogram

Milk: $7.0 \mathrm{E} 7$ liter

Meat: $8.1 E 7$ kilogram

1

0.8

Meteorology Period of Record: 1 JUN 70 - 31 AlJG 74 Recovery: $87 \%$

SITE-SPECIFIC DATA - WATERBORNE PATHWAYS via ALTAMAHA RIVER

Average River Flow

at Site: $13,000 \mathrm{ft}^{3} / \mathrm{s}$

Drinking Water:

Exposed Population: None

Fish:

Edible Harvest: $6.3 \mathrm{E} 5(\mathrm{a}) \mathrm{kg} / \mathrm{yr}$

Dilution Factor: 1

(a) Commercial catch plus 3 pounds of game fish per year taken from river by average person according to FES (1972). 


\section{POPULATION DOSE-COMMITMENT ESTIMATES AND AVERAGE INDIVIDUAL DOSE-COMHITMENT HISTOGRAM FOR}

E. I. HATCH 1 AND 2

Dose Commitments (person-rem) from Waterborne Pathways

Iotal Body GI-LLI $\underline{\text { Thyroid }}$ Bone Liver

$\begin{array}{llllll}\text { Infant } & 0.0 \mathrm{E}+00 & 0.0 \mathrm{E}+00 & 0.0 \mathrm{E}+00 & 0.0 \mathrm{E}+00 & 0.0 \mathrm{E}+00 \\ \text { Child } & 1.5 \mathrm{E}-01 & 1.1 \mathrm{E}-02 & 2.1 \mathrm{E}-03 & 6.8 \mathrm{E}-01 & 8.0 \mathrm{E}-01 \\ \text { Teen } & 2.7 \mathrm{E}-01 & 2.2 \mathrm{E}-02 & 1.5 \mathrm{E}-03 & 4.1 \mathrm{E}-01 & 6.8 \mathrm{E}-01 \\ \text { Adult } & 2.9 \mathrm{E}+00 & 2.0 \mathrm{E}-01 & 1.0 \mathrm{E}-02 & 2.4 \mathrm{E}+00 & 4.0 \mathrm{E}+00 \\ \text { TOTAL } & 3.3 \mathrm{E}+00 & 2.3 \mathrm{E}-01 & 1.4 \mathrm{E}-02 & 3.5 \mathrm{E}+00 & 5.5 \mathrm{E}+00\end{array}$

Dose Commitments (person-rem) from Airborne Pathways

Iotal Body GI-LLI Thyroid Bone Liver Lung

$\begin{array}{lllllll}\text { Infant } & 1.2 \mathrm{E}-03 & 1.2 \mathrm{E}-03 & 3.2 \mathrm{E}-03 & 1.3 \mathrm{E}-03 & 1.3 \mathrm{E}-03 & 1.3 \mathrm{E}-03 \\ \text { Child } & 1.4 \mathrm{E}-02 & 1.4 \mathrm{E}-02 & 2.4 \mathrm{E}-02 & 1.4 \mathrm{E}-02 & 1.4 \mathrm{E}-02 & 1.5 \mathrm{E}-02 \\ \text { Teen } & 1.0 \mathrm{E}-02 & 1.0 \mathrm{E}-02 & 1.4 \mathrm{E}-02 & 1.0 \mathrm{E}-02 & 1.0 \mathrm{E}-02 & 1.2 \mathrm{E}-02 \\ \text { Adult } & 6.1 \mathrm{E}-02 & 6.1 \mathrm{E}-02 & 7.4 \mathrm{E}-02 & 6.02 \mathrm{E}-02 & 6.1 \mathrm{E}-02 & 6.7 \mathrm{E}-02 \\ \text { TOTAL } & 8.6 \mathrm{E}-02 & 8.6 \mathrm{E}-02 & 1.2 \mathrm{E}-01 & 8.5 \mathrm{E}-02 & 8.7 \mathrm{E}-02 & 9.6 \mathrm{E}-02\end{array}$

Production/Consumption factors:

Produce: 0.14 Milk: 1.6 Meat: 3.0

Fraction of Population Receiving an Average Individual Total-Body Dose Commitment from Airborne Pathways

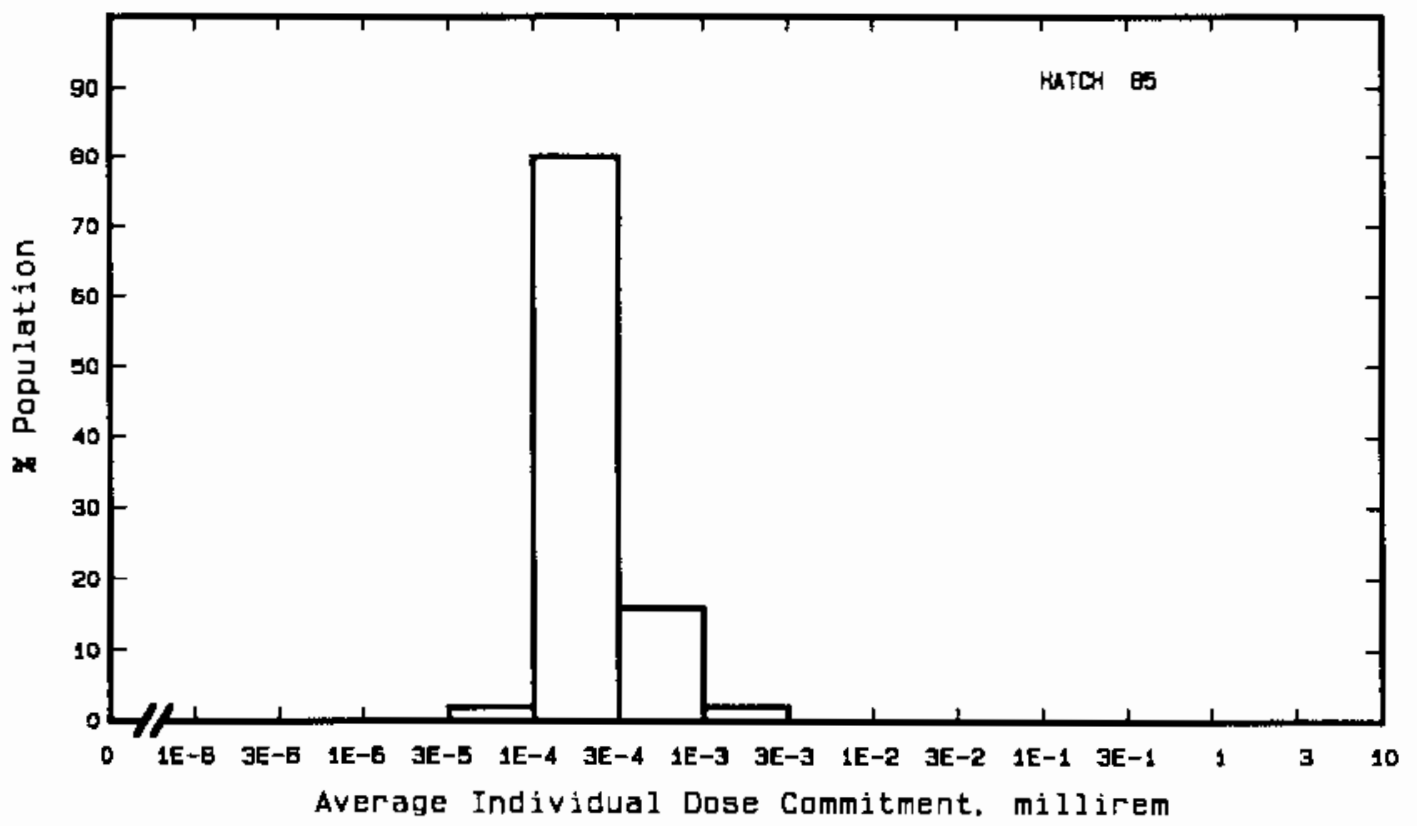


Site: INDIAN POINT

BUCHANAN, NEW YORK

Location: $\quad \mathrm{N} 41.2714^{\circ}$ W $73.9525^{\circ}$

POPULATION DATA

Total Population Within 2-to-80-km Region: 1.5E7

Major Metropolitan Centers Within Region:

Center

New York SMSA

Newark SMSA

Nassau County SMSA

Jersey City SMSA

Paterson-Clifton-Passaic SMSA
Population

$8,500,000$

$1,900,000$

$1,300,000$

560,000

450,000
Location

$57 \mathrm{~km} \mathrm{~S}$

$62 \mathrm{~km} \quad \mathrm{SSW}$

$70 \mathrm{~km} \quad$ SSE

$61 \mathrm{~km} \quad \mathrm{~S}$

$44 \mathrm{~km} \quad \mathrm{SSW}$

SITE-SPECIFIC DATA - AIRBORNE PATHWAYS

Average Annual State Production

of Crops and Animal Products

In $80-\mathrm{km}$ Radius Circle

Regional Productivity Factor:

Animal Grazing Factor:

Meteorology Period of Record: 1 JAN 75 - 31 DEC 75 Recovery: 96\%
Veg: $7.6 \mathrm{E} 7 \mathrm{kilogram}$

Milk: 7.0E8 liter

Meat: $3.3 \mathrm{E} 7 \mathrm{kilogram}$

0.8

0.5

SITE-SPECIFIC DATA - WATERBORNE PATHWAYS via HUDSON RIVER

Average River $\mathrm{Flow}$

at Site: $20,000 \mathrm{ft}^{3} / \mathrm{s}$

Drinking Water: Exposed Population: None

Fish:

Edible Harvest: (a)

Dilution Factor: $0.001^{(b)}$

(a) No fish catch data given in FES (1982), so generic consumption rates used (Table A-1).

(b) One percent of population obtain 10\% of their fish from river according to FES (1972). 


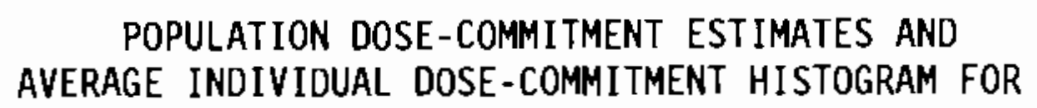

INDIAN POINT 1,2 AND 3

Dose Commitments (person-rem) from Waterborne Pathways

Total Body GI-LLI $\underline{\text { Thyroid Bone }}$

$\begin{array}{llllll}\text { Infant } & 0.0 \mathrm{E}+00 & 0.0 \mathrm{E}+00 & 0.0 \mathrm{E}+00 & 0.0 \mathrm{E}+00 & 0.0 \mathrm{E}+00 \\ \text { Child } & 2.4 \mathrm{E}-02 & 1.3 \mathrm{E}-03 & 3.1 \mathrm{E}-03 & 1.2 \mathrm{E}-01 & 1.4 \mathrm{E}-01 \\ \text { Teen } & 4.6 \mathrm{E}-02 & 2.6 \mathrm{E}-03 & 2.2 \mathrm{E}-03 & 7.5 \mathrm{E}-02 & 1.2 \mathrm{E}-01 \\ \text { Adult } & 5.0 \mathrm{E}-01 & 2.1 \mathrm{E}-02 & 1.5 \mathrm{E}-02 & 4.3 \mathrm{E}-01 & 6.9 \mathrm{E}-01 \\ \text { TOTAL } & 5.7 \mathrm{E}-01 & 2.5 \mathrm{E}-02 & 2.0 \mathrm{E}-02 & 6.3 \mathrm{E}-01 & 9.5 \mathrm{E}-01\end{array}$

Dose Commitments (person-rem) from Airborne Pathways

Total Body GI-LLI Thyroid Bone Liver

$\begin{array}{lllllll}\text { Infant } & 5.0 \mathrm{E}-02 & 5.0 \mathrm{E}-02 & 5.9 \mathrm{E}-02 & 1.4 \mathrm{E}-01 & 5.0 \mathrm{E}-02 & 5.2 \mathrm{E}-02 \\ \text { Child } & 4.5 \mathrm{E}-01 & 4.5 \mathrm{E}-01 & 5.1 \mathrm{E}+01 & 1.1 \mathrm{E}+00 & 4.5 \mathrm{E}-01 & 4.7 \mathrm{E}-01 \\ \text { Teen } & 2.6 \mathrm{E}-01 & 2.6 \mathrm{E}-01 & 2.9 \mathrm{E}-01 & 4.7 \mathrm{E}-01 & 2.6 \mathrm{E}-01 & 2.9 \mathrm{E}-01 \\ \text { Adult } & 1.4 \mathrm{E}+00 & 1.4 \mathrm{E}+00 & 1.5 \mathrm{E}+00 & 2.0 \mathrm{E}+00 & 1.4 \mathrm{E}+00 & 1.5 \mathrm{E}+00 \\ \text { TOTAL } & 2.2 \mathrm{E}+00 & 2.2 \mathrm{E}+00 & 2.4 \mathrm{E}+00 & 3.7 \mathrm{E}+00 & 2.2 \mathrm{E}+00 & 2.3 \mathrm{E}+00\end{array}$

Production/Consumption factors:

Produce: $0.020 \quad$ Milk: 0.28 Meat: 0.021

Fraction of Population Receiving an Average Individual

Total-Body Dose Commitment from Airborne Pathways

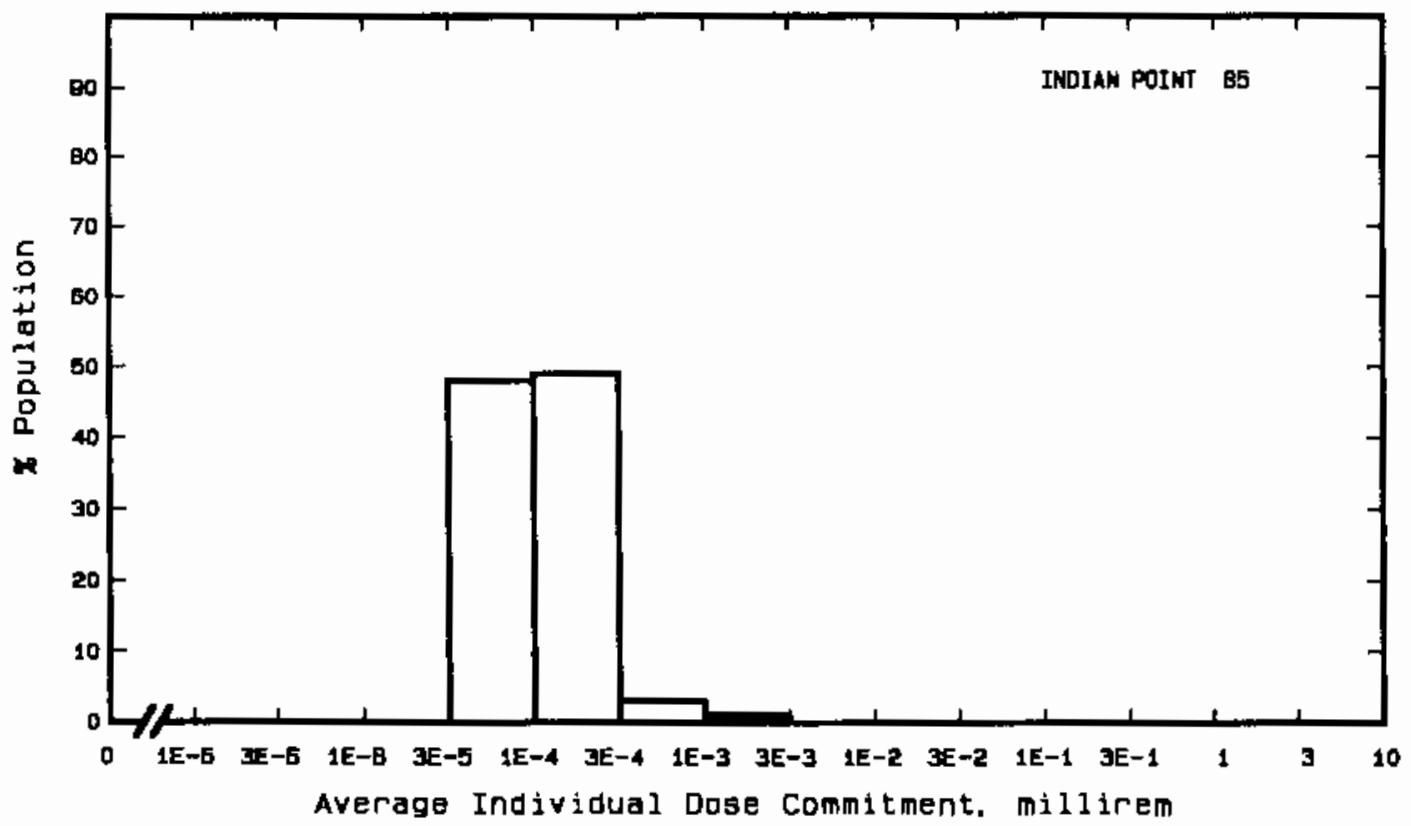


Site: KEWAUNEE

Location: $\quad$ N $44.3431^{\circ}$
CARLTON, WISCONSIN

W $87.5361^{\circ}$

POPULATION DATA

Total Population Within 2-to-80-km Region: 5.2E5

Major Metropolitan Centers Within Region:

Center
Greenbay SMSA
Appleton SMSA
Sheboygan
Manitowoc
Neenah

Population

180,000
290,000
48,000
33,000
22,000

\section{Location}

$\begin{array}{lll}44 & \mathrm{~km} & \mathrm{NW} \\ 72 & \mathrm{~km} & W \\ 65 & \mathrm{~km} & \text { SSW } \\ 29 & \mathrm{~km} & \text { SSW } \\ 72 & \mathrm{~km} & W\end{array}$

\section{SITE-SPECIFIC DATA - AIRBORNE PATHWAYS}

Average Annual State Production

of Crops and Animal Products

In $80-\mathrm{km}$ Radius Circle

Regional Productivity Factor:

Animal Grazing Factor:
Veg: $7.2 E 7$ kilogram

Milk: 1.2Eg liter

Meat: $1.0 \mathrm{E} 8 \mathrm{kilogram}$

0.5

0.5

Meteorology Period of Record: 2 JAN 69 - 31 [IEC 69 Recovery: $76 \%$

SITE-SPECIFIC DATA - WATERBORNE PATHWAYS via LAKE MICHIGAN

Average 0ilution Flow from P] ant: $60 \mathrm{ft}^{3} / \mathrm{s}$

Orinking water:

Exposed Population: 260,000 Dilution Factor: $8.2 \mathrm{E}-3(\mathrm{a})$

Fish:

Edible Harvest: $1.1^{(\mathrm{b})} \mathrm{kg} / \mathrm{yr}$
Dilution Factor: $0.01{ }^{(\mathrm{c})}$

(a) Drinking water dilution factor estimated by averaging dilution factors derived from FES (1972) suitably weighted for populations.

(b) Average individual consumption rate as given in FES used in lieu of catch data.

(c) Dilution factor reduced $1 / 10$ from that used in FES in consideration of lake mixing. 
POPULATION DOSE-COMMITMENT ESTIMATES AND
AVERAGE INDIVIOUAL DOSE-COMMITMENT HISTOGRAM FOR

KEWAUNEE

Dose Commitments (person-rem) from Waterborne Pathways

Total Body GI-LLI $\underline{\text { Thyroid }}$ Bone

$\begin{array}{llllll}\text { Infant } & 1.3 \mathrm{E}-02 & 1.3 \mathrm{E}-02 & 1.3 \mathrm{E}-02 & 2.4 \mathrm{E}-03 & 1.4 \mathrm{E}-02 \\ \text { Child } & 1.7 \mathrm{E}-01 & 2.2 \mathrm{E}-01 & 1.4 \mathrm{E}-01 & 1.6 \mathrm{E}-01 & 2.9 \mathrm{E}-01 \\ \text { Teen } & 1.0 \mathrm{E}-01 & 2.0 \mathrm{E}-01 & 5.2 \mathrm{E}-02 & 8.8 \mathrm{E}-02 & 1.7 \mathrm{E}-01 \\ \text { Adult } & 9.6 \mathrm{E}-01 & 1.7 \mathrm{E}+00 & 4.4 \mathrm{E}-01 & 5.1 \mathrm{E}-01 & 1.2 \mathrm{E}+00 \\ \text { TOTAL } & 1.2 \mathrm{E}+00 & 2.2 \mathrm{E}+00 & 6.4 \mathrm{E}-01 & 7.7 \mathrm{E}-01 & 1.6 \mathrm{E}+00\end{array}$

Dose Commitments (person-rem) from Airborne Pathways

Total Body GI-LLI Ihyroid $\underline{\text { 8one }}$ Liver

$\begin{array}{lllllll}\text { Infant } & 3.5 \mathrm{E}-05 & 3.5 \mathrm{E}-05 & 4.0 \mathrm{E}-05 & 3.9 \mathrm{E}-06 & 3.5 \mathrm{E}-05 & 3.6 \mathrm{E}-05 \\ \text { Child } & 5.4 \mathrm{E}-04 & 5.4 \mathrm{E}-04 & 5.8 \mathrm{E}-04 & 4.9 \mathrm{E}-05 & 5.4 \mathrm{E}-04 & 5.5 \mathrm{E}-04 \\ \text { Teen } & 3.3 \mathrm{E}-04 & 3.3 \mathrm{E}-04 & 3.5 \mathrm{E}-04 & 3.4 \mathrm{E}-05 & 3.3 \mathrm{E}-04 & 3.4 \mathrm{E}-04 \\ \text { Adult } & 1.8 \mathrm{E}-03 & 1.8 \mathrm{E}-03 & 1.9 \mathrm{E}-03 & 2.0 \mathrm{E}-04 & 1.8 \mathrm{E}-03 & 1.9 \mathrm{E}-03 \\ \text { TOTAL } & 2.7 \mathrm{E}-03 & 2.7 \mathrm{E}-03 & 2.9 \mathrm{E}-03 & 2.8 \mathrm{E}-04 & 2.7 \mathrm{E}-03 & 2.8 \mathrm{E}-03\end{array}$

Production/Consumption factors:

Produce: 0.30

Milk: 7.2

Meat: 1.0

Fraction of Population Recelving an Average Individual Total-Body Dose Commitment from Airborne Pathways

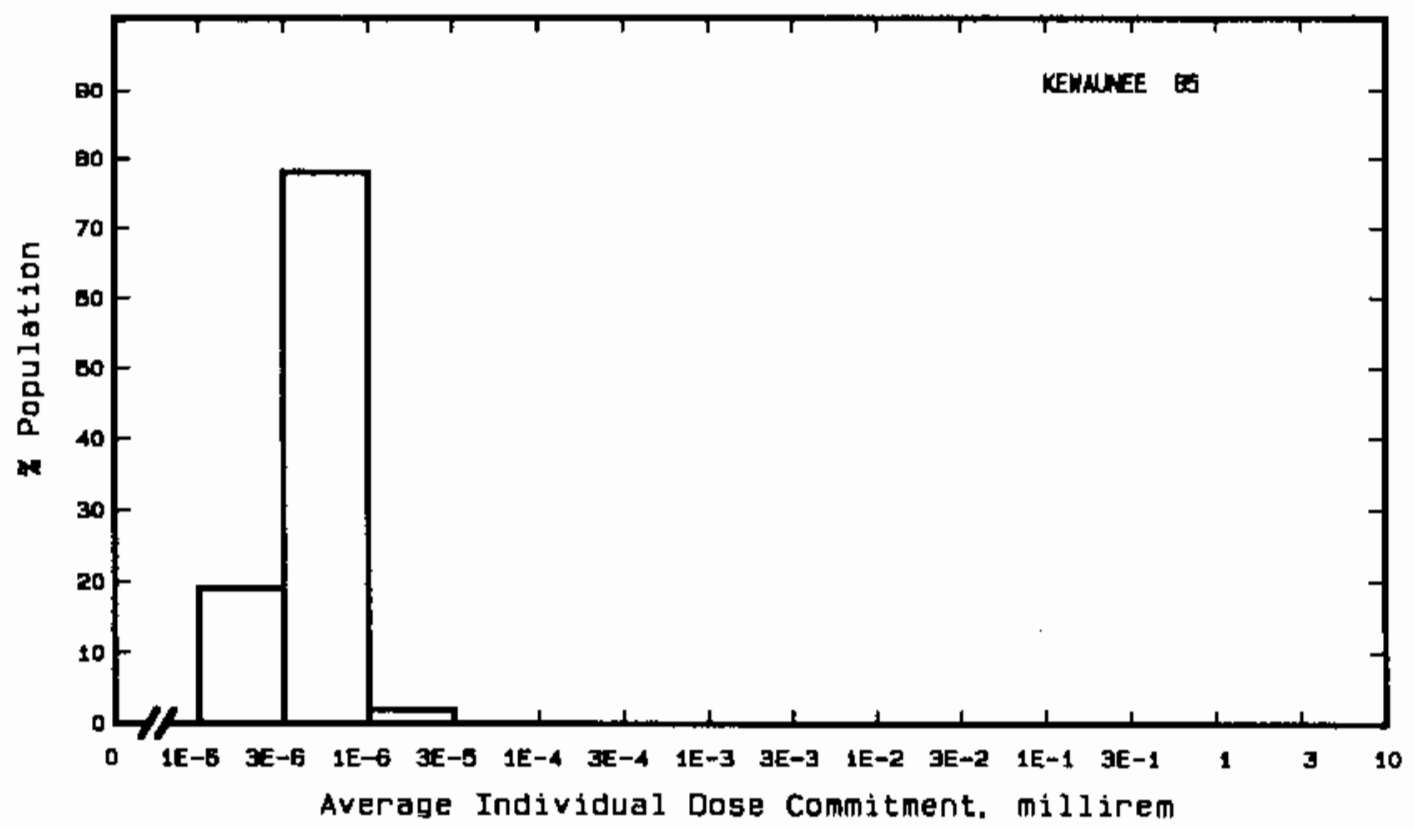


Site: LACROSSE

Location: $\quad$ N $43.5583^{\circ} \quad$ W $91.2306^{\circ}$

POPULATION DATA

Total Population Within 2-to-80-km Region: 3.5E5

Major Metropolitan Centers Within Region:

\section{Center}

La Crosse SMSA

Winona
Population

92,000

25,000
Location

$27 \mathrm{~km} \mathrm{~N}$

$64 \mathrm{~km} \quad W N W$

\section{SITE-SPECIFIC OATA - AIRBORNE PATHWAYS}

Average Annual State Production

of Crops and Animal Products

In 80-km Radius Circle

Regiona1 Productivity Factor:

Anima1 Grazing Factor:
Veg: $7.2 E 7$ kilogram

Milk: 1.2E9 liter

Meat: $1.0 \mathrm{E} 8$ kilogram

1

0.5

Meteorology Period of Record: 1 JAN 75 - 31 DEC 75 Recovery: 97\%

Average River

Flow at Site: $28,000 \mathrm{ft}^{3} / \mathrm{s}$

Drinking Water:

Exposed Population: None

Fish:

Edible Harvest: (a)

Dilution Factor: 0.5 (a)

(a) No fish catch data given in FES (1976), so $1 / 2$ population assumed to consume river fish at generic consumption rates (Table A-1). 
POPULATION DOSE-COMMITMENT ESTIMATES AND

AVERAGE INDIVIDUAL DOSE-COMMITMENT HISTOGRAM FOR

LACROSSE

Dose Commitments (person-rem) from Waterborne Pathways

Tota] Body GI-LLI $\underline{\text { Thyroid }}$ Bone Liver

$\begin{array}{llllll}\text { Infant } & 0.0 \mathrm{E}+00 & 0.0 \mathrm{E}+00 & 0.0 \mathrm{E}+00 & 0.0 \mathrm{E}+00 & 0.0 \mathrm{E}+00 \\ \text { Child } & 2.4 \mathrm{E}-02 & 7.5 \mathrm{E}-03 & 8.0 \mathrm{E}-03 & 1.4 \mathrm{E}-01 & 1.4 \mathrm{E}-01 \\ \text { Teen } & 4.2 \mathrm{E}-02 & 1.6 \mathrm{E}-02 & 5.8 \mathrm{E}-03 & 8.6 \mathrm{E}-02 & 1.1 \mathrm{E}-01 \\ \text { Adult } & 4.5 \mathrm{E}-01 & 1.4 \mathrm{E}-01 & 3.8 \mathrm{E}-02 & 5.0 \mathrm{E}-01 & 6.7 \mathrm{E}-01 \\ \text { TOTAL } & 5.2 \mathrm{E}-01 & 1.6 \mathrm{E}-01 & 5.2 \mathrm{E}-02 & 7.3 \mathrm{E}-01 & 9.2 \mathrm{E}-01\end{array}$

Dose Commitments (person-rem) from Airborne Pathways

Total Body GI-LLI Ihyroid Bone Liver

$\begin{array}{lllllll}\text { Infant } & 7.6 \mathrm{E}-03 & 7.6 \mathrm{E}-03 & 8.4 \mathrm{E}-03 & 7.5 \mathrm{E}-03 & 7.6 \mathrm{E}-03 & 7.8 \mathrm{E}-03 \\ \text { Chi1d } & 8.5 \mathrm{E}-02 & 8.5 \mathrm{E}-02 & 9.4 \mathrm{E}-02 & 8.3 \mathrm{E}-02 & 8.5 \mathrm{E}-02 & 8.8 \mathrm{E}-02 \\ \text { Teen } & 6.2 \mathrm{E}-02 & 6.2 \mathrm{E}-02 & 6.5 \mathrm{E}-02 & 6.1 \mathrm{E}-02 & 6.2 \mathrm{E}-02 & 6.6 \mathrm{E}-02 \\ \text { Adult } & 3.7 \mathrm{E}-01 & 3.7 \mathrm{E}-01 & 3.9 \mathrm{E}-01 & 3.7 \mathrm{E}-01 & 3.7 \mathrm{E}-01 & 3.9 \mathrm{E}-01 \\ \text { TOTAL } & 5.3 \mathrm{E}-01 & 5.3 \mathrm{E}-01 & 5.6 \mathrm{E}-01 & 5.2 \mathrm{E}-01 & 5.3 \mathrm{E}-01 & 5.5 \mathrm{E}-01\end{array}$

Production/Consumption factors:

Produce: 1.1 Milk: $26 \quad$ Meat: 3.7

Fraction of Population Receiving an Average Individual

Total-Body Oose Commitment from Airborne Pathways

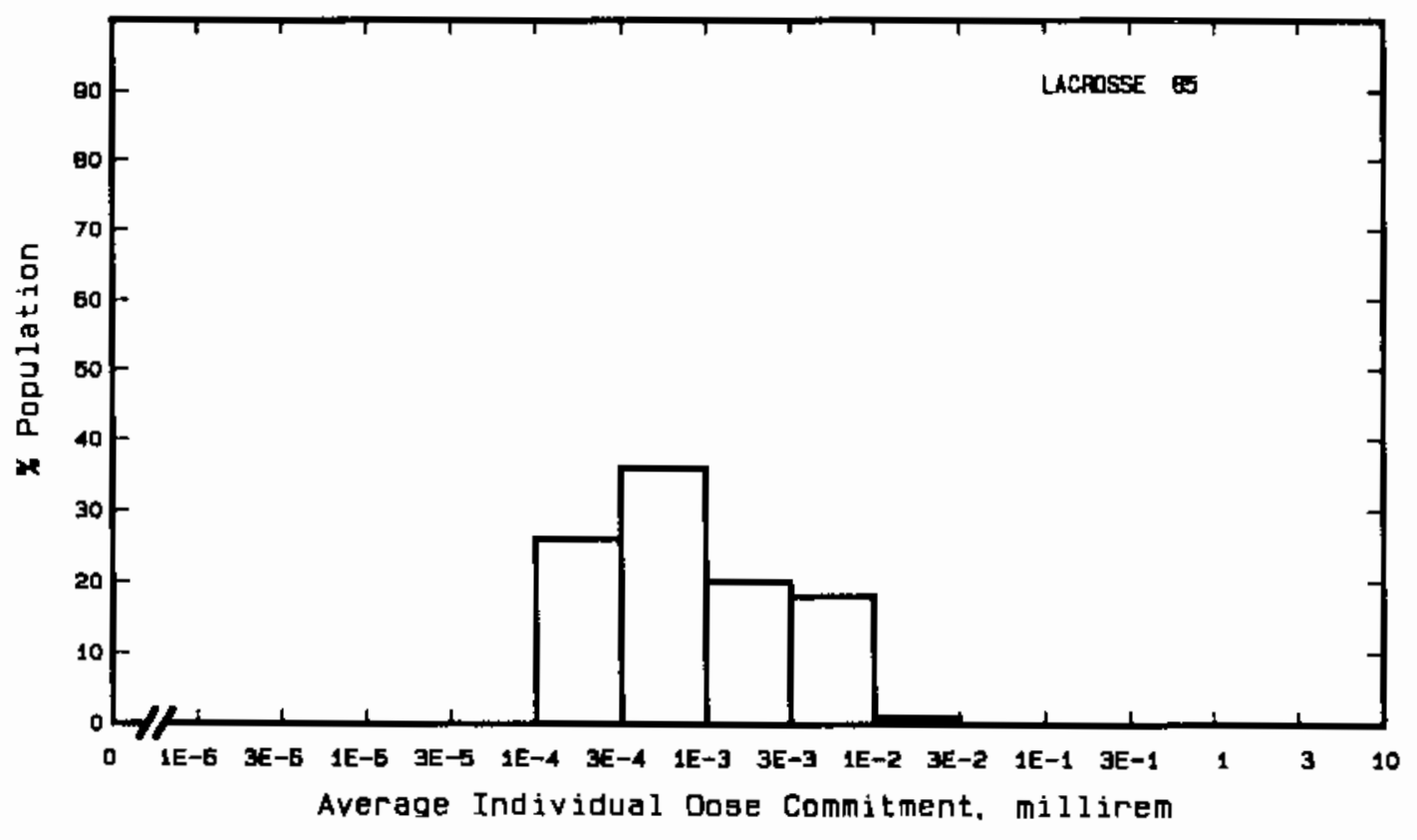


Site: LASALLE

SENECA, ILLINOIS

Location: $\quad$ N $41.2439^{\circ} \quad$ W $88.6708^{\circ}$

POPULATION DATA

Total Population Within 2-to-80-km Region: 1.0E6

Major Metropolitan Centers Within Region:

Center

Joliet

Aurora

Kankakee

Dekalb

Naperville
Population

78,000

80,000

103,000

33,000

42,000
Location

$59 \mathrm{~km} \quad \mathrm{NNE}$

$65 \mathrm{~km} \quad \mathrm{NNE}$

$69 \mathrm{~km} \quad \mathrm{ESE}$

$77 \mathrm{~km} \quad \mathrm{~N}$

$73 \mathrm{~km} \quad \mathrm{NE}$

SITE-SPECIFIC DATA - AIRBORNE PATHWAYS

Average Annual State Production

of Crops and Animal Products

In 80-km Radius Circle

Regional Productivity Factor:

Animal Grazing Factor:
Veg: 1.1 E8 kilogram

Milk: 1.8E8 liter

Meat: $1.9 E 8 \mathrm{ki}$ logram

Meteorology Period of Record: 1 Jan $82-31$ Dec 82 Recovery: $99 \%$

SITE-SPECIFIC DATA - WATERBORNE PATHWAYS via ILLINOIS RIVER

Average River Flow

at site: $11,000 \mathrm{ft}^{3} / \mathrm{s}$

Drinking Water:

Exposed Population: None

Dilution Factor:

Fish:

Edible Harvest: None(a)

Dilution Factor:

(a) Because of river quality, no fish consumption is considered according to FES (1973). 


\section{POPULATION DOSE-COMMITMENT ESTIMATES AND}

AVERAGE INDIVIDUAL DOSE-COMMITMENT HISTOGRAM FOR

LASALLE 1 and 2

Dose Commitments (person-rem) from Waterborne Pathways

Total Body GI-LII Ihyroid Bone Liver

Infant

Child

Teen

Adult

(Little or No Waterborne Pathway Doses)

TOTAL

Dose Commitments (person-rem) from Airborne Pathways

Total Body GI-LII Ihyroid Bone Liver

$\begin{array}{lllllll}\text { Infant } & 9.9 \mathrm{E}-04 & 9.8 \mathrm{E}-04 & 6.1 \mathrm{E}-03 & 9.7 \mathrm{E}-04 & 9.9 \mathrm{E}-04 & 1.4 \mathrm{E}-03 \\ \text { Child } & 1.3 \mathrm{E}-02 & 1.4 \mathrm{E}-02 & 5.1 \mathrm{E}-02 & 1.1 \mathrm{E}-02 & 1.2 \mathrm{E}-02 & 1.8 \mathrm{E}-02 \\ \text { Teen } & 8.5 \mathrm{E}-03 & 1.2 \mathrm{E}-02 & 2.4 \mathrm{E}-02 & 7.8 \mathrm{E}-03 & 8.2 \mathrm{E}-03 & 1.5 \mathrm{E}-02 \\ \text { Adult } & 5.0 \mathrm{E}-02 & 7.5 \mathrm{E}-02 & 1.0 \mathrm{E}-01 & 4.7 \mathrm{E}-02 & 4.9 \mathrm{E}-02 & 7.6 \mathrm{E}-02 \\ \text { TOTAL } & 7.3 \mathrm{E}-02 & 1.0 \mathrm{E}-01 & 1.9 \mathrm{E}-01 & 6.6 \mathrm{E}-02 & 7.0 \mathrm{E}-02 & 1.1 \mathrm{E}-01\end{array}$

Production/Consumption factors:

Produce: $0.50 \quad$ Milk: $1.2 \quad$ Meat: 2.0

Fraction of Population Receiving an Average Individual

Total-Budy Dose Commitment from Airborne Pathways

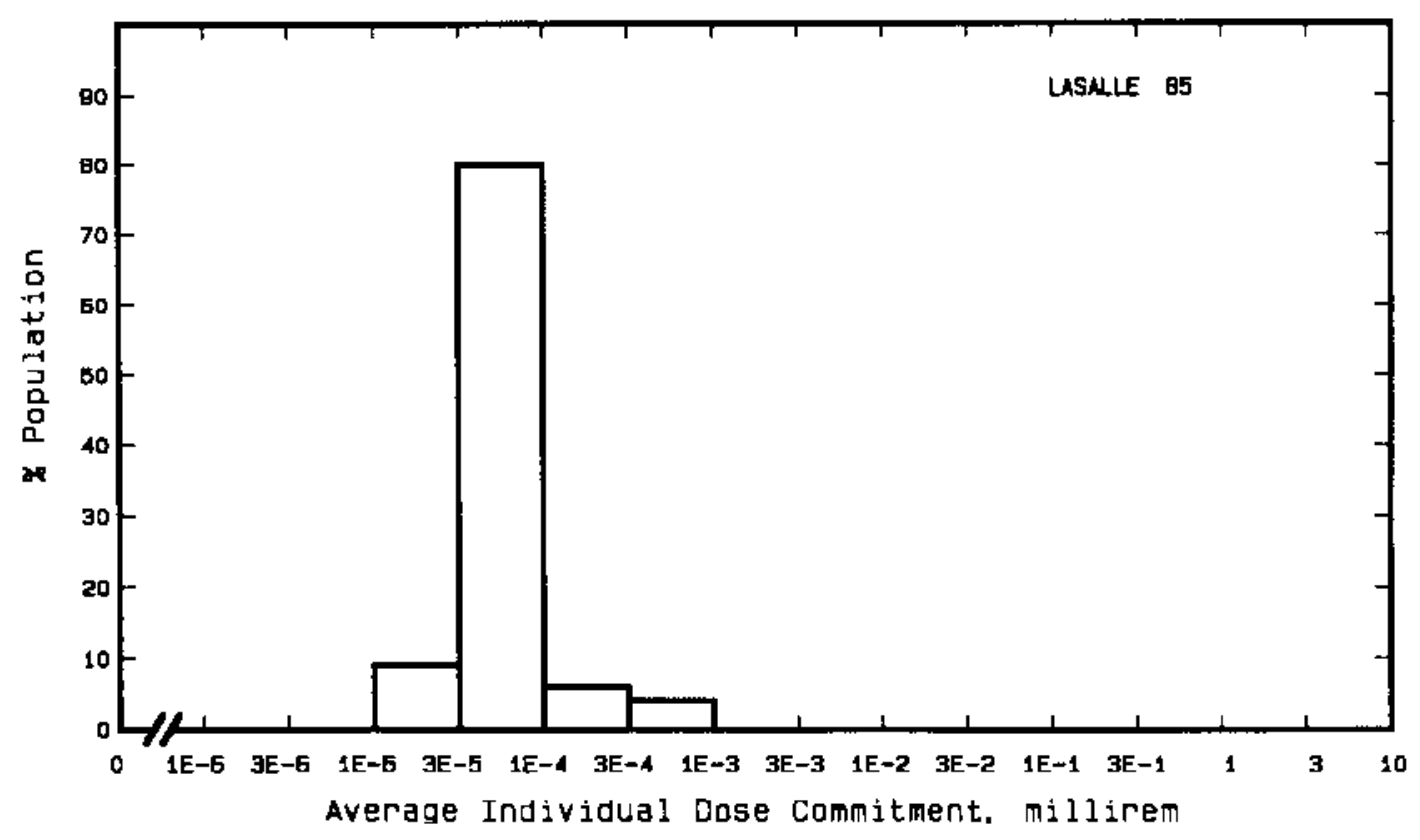


Site: LIMERICK

Location: $\quad$ N $40.2242^{\circ}$
POTTSTONN, PENNSYLVANIA

W $75.5875^{\circ}$

POPULATION DATA

Total Population Within 2-to-80-km Region: $6.8 \mathrm{E} 6$

Major Metropolitan Centers Within Region:

Center

Philadelphia PMSA

Allentown-Bethlehem MSA

Reading MSA

Lancaster MSA

Wi]mington PMSA

Camion

Trenton PMSA
Population

$4,800,000$

65,000

310,000

360,000

540,000

110,000

310,000

\section{Location}

$40 \mathrm{~km} \quad \mathrm{ESE}$

$42 \mathrm{~km}$ ENE

$34 \mathrm{~km} \quad W N W$

$68 \mathrm{~km} \quad W S W$

$56 \mathrm{~km} \quad \mathrm{~S}$

$52 \mathrm{~km} \quad \mathrm{SE}$

$71 \mathrm{~km} \quad \mathrm{E}$

SITE-SPECIFIC DATA - AIRBORNE PATHWAYS

Average Annual State Production

of Crops and Animal Products

In 80-km Radius Circle

Regional Productivity Factor:

Animal Grazing Factor:
Veg: $5.3 \mathrm{E} 7 \mathrm{ki}$ logram

Mi7k: 5.3E8 liter

Meat: 5.4E7 kilogram

0.9

0.6

Meteorology Period of Record: 1 Jan $74-31$ Dec 74 Recovery: 93\%

Average River Flow at site: $3,000 \mathrm{ft}^{3} \mathrm{~s}$

Drinking Water:

Fish:
Exposed Population: $3,000,000$

Dilution Factor: 1

Edible Harvest: None 


\section{POPULATION DOSE-COMMITMENT ESTIMATES AND \\ AVERAGE INDIVIDUAL DOSE-COMMITMENT HISTOGRAM FOR \\ LIMERICK}

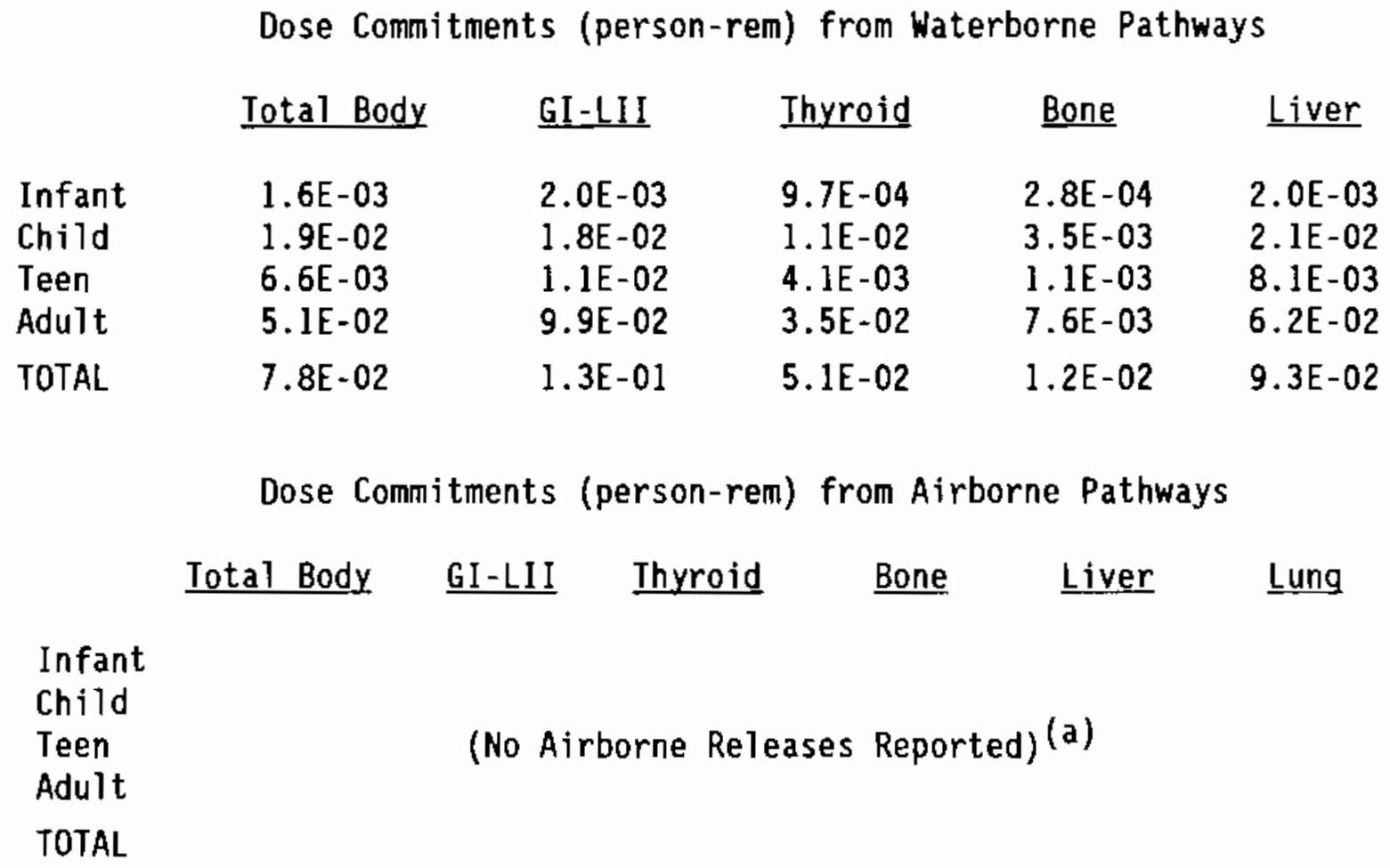

(a) Tichler, et al. 1988. 
Site: MAINE YANKEE

Location: N $43.9506^{\circ}$
LINCOLN COUNTY, MAINE

W $69.6961^{\circ}$

POPULATION DATA

Total Population Within 2-to-80-km Region: 6.1E5

Major Metropolitan Centers Within Region:

\section{Center}

Portland SMSA

Lewiston-Auburn SMSA

Augusta

Biddeford

Waterville
Population

180,000

73,000

22,000

20,000

18,000
Location

$56 \mathrm{~km} \quad W S W$

$45 \mathrm{~km} \quad W N W$

$41 \mathrm{~km} \quad \mathrm{~N}$

$80 \mathrm{~km} \quad \mathrm{SW}$

$67 \mathrm{~km} \mathrm{~N}$

SITE-SPECIFIC DATA - AIRBORNE PATHWAYS

Average Annual State Production

of Crops and Animal Products

In $80-\mathrm{km}$ Radius Circle

Regional Productivity Factor:

Animal Grazing Factor:
Veg: $2.4 \mathrm{E} 8$ kilogram

Milk: 6.6E7 liter

Meat: 4.3E6 kilogram

0.6

0.5

Meteorology Period of Record: 1 APR 75 - 31 MAR 76 Recovery: 98\%

Average Dilution Flow

from Plant: $790 \mathrm{ft}^{3} / \mathrm{s}$

Fish:

Edible Harvest: (a)

Dilution Factor: 0.001

Invertebrates: Edible Harvest: (a)

Dilution Factor: 0.002

(a) No seafood harvest data given in FES (1972), thus generic population consumption rates used (Table A-1). 
POPULATION DOSE-COMMITMENT ESTIMATES AND
AVERAGE INDIVIDUAL DOSE-COMMITMENT HISTOGRAM FOR

MAINE YANKEE

Dose Commitments (person-rem) from Waterborne Pathways

Total Body GI-LLI Ihyroid Bone Liver

$\begin{array}{llllll}\text { Infant } & 0.0 \mathrm{E}+00 & 0.0 \mathrm{E}+00 & 0.0 \mathrm{E}+00 & 0.0 \mathrm{E}+00 & 0.0 \mathrm{E}+00 \\ \text { Child } & 3.1 \mathrm{E}-05 & 4.2 \mathrm{E}-05 & 2.7 \mathrm{E}-04 & 1.6 \mathrm{E}-05 & 3.4 \mathrm{E}-05 \\ \text { Teen } & 2.7 \mathrm{E}-05 & 7.3 \mathrm{E}-05 & 2.0 \mathrm{E}-04 & 9.5 \mathrm{E}-06 & 2.9 \mathrm{E}-05 \\ \text { Adult } & 2.1 \mathrm{E}-04 & 6.4 \mathrm{E}-04 & 1.3 \mathrm{E}-03 & 5.4 \mathrm{E}-05 & 2.0 \mathrm{E}-04 \\ \text { TOTAL } & 2.6 \mathrm{E}-04 & 7.5 \mathrm{E}-04 & 1.8 \mathrm{E}-03 & 8.0 \mathrm{E}-05 & 2.6 \mathrm{E}-04\end{array}$

Dose Commitments (person-rem) from Airborne Pathways

Iotal Body GI-LLI Ihyroid Bone Liver Lung

$\begin{array}{lllllll}\text { Infant } & 1.5 \mathrm{E}-04 & 1.5 \mathrm{E}-04 & 2.0 \mathrm{E}-04 & 1.4 \mathrm{E}-04 & 1.5 \mathrm{E}-04 & 1.6 \mathrm{E}-04 \\ \text { Child } & 1.8 \mathrm{E}-03 & 1.8 \mathrm{E}-03 & 2.6 \mathrm{E}-03 & 1.5 \mathrm{E}-03 & 1.8 \mathrm{E}-03 & 2.0 \mathrm{E}-03 \\ \text { Teen } & 1.3 \mathrm{E}-03 & 1.3 \mathrm{E}-03 & 1.6 \mathrm{E}-03 & 1.1 \mathrm{E}-03 & 1.3 \mathrm{E}-03 & 1.5 \mathrm{E}-03 \\ \text { Adult } & 7.6 \mathrm{E}-03 & 7.7 \mathrm{E}-03 & 9.0 \mathrm{E}-03 & 6.8 \mathrm{E}-03 & 7.6 \mathrm{E}-03 & 8.3 \mathrm{E}-03 \\ \text { TOTAL } & 1.1 \mathrm{E}-02 & 1.1 \mathrm{E}-02 & 1.3 \mathrm{E}-02 & 9.6 \mathrm{E}-03 & 1.1 \mathrm{E}-02 & 1.2 \mathrm{E}-02\end{array}$

Production/Consumption factors:

Produce: 1.2 Milk: 0.49 Meat: 0.053

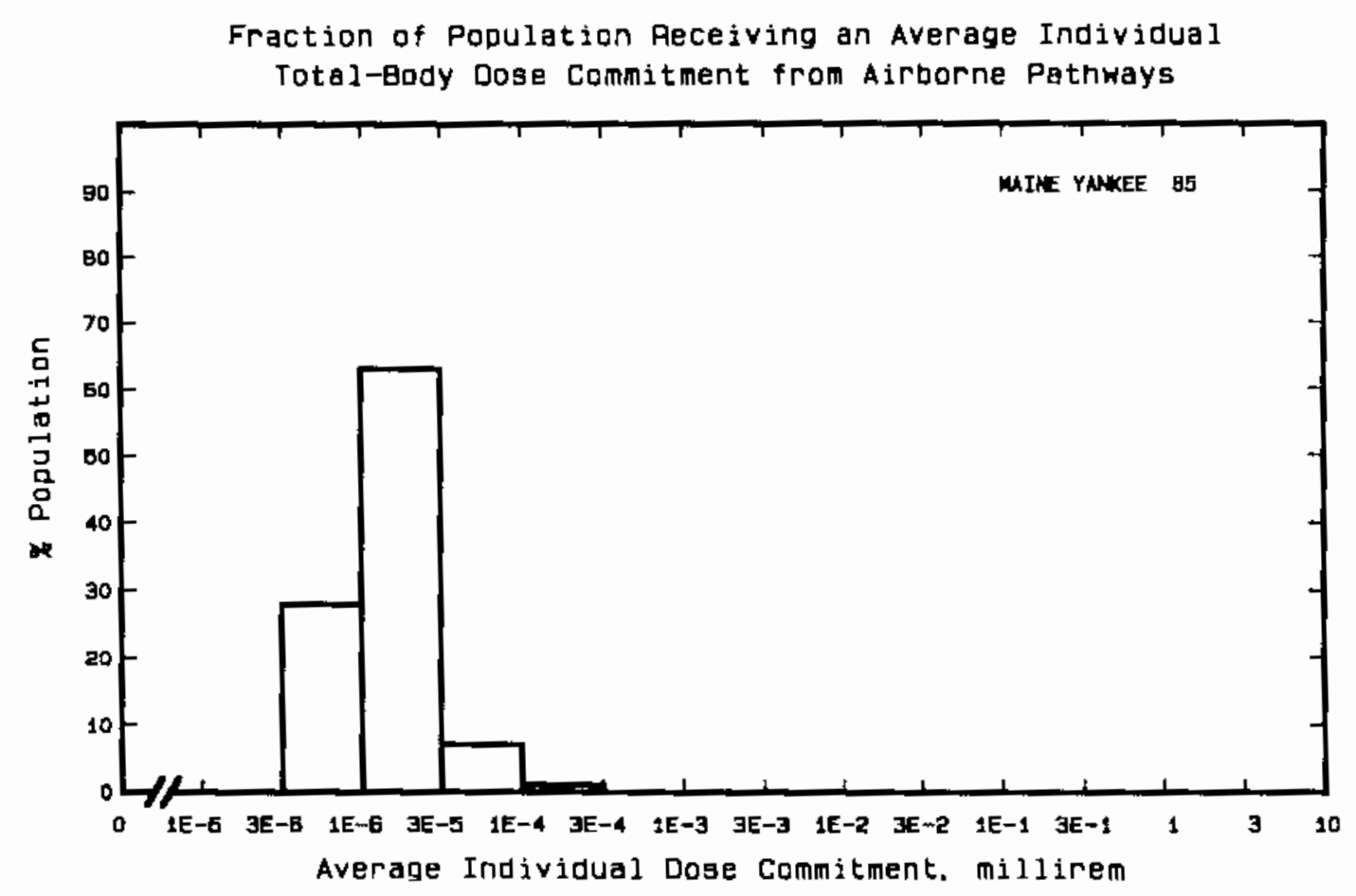


Site: McGUIRE

Location: $\quad$ N $35.4322^{\circ}$ W $80.9483^{\circ}$

POPULATION DATA

Total Population Within 2-to-80-km Region: 1.7 E6

Major Metropolitan Centers Within Region:

Center

Charlotte-Gastonia SMSA 660,000

Rockhill

Kannapolis

Sal i sbury

Hickory
Population

36,000

36,000

24,000

22,000
Location

$25 \mathrm{~km} \mathrm{~S}$

$57 \mathrm{~km} \quad \mathrm{~S}$

$30 \mathrm{~km} \quad \mathrm{E}$

$51 \mathrm{~km}$ ENE

$49 \mathrm{~km} \quad \mathrm{NW}$

SITE-SPECIFIC DATA - AIRBORNE PATHWAYS

Average Annual State Production

of Crops and Animal Products

In $80-\mathrm{km}$ Radius Circle

Regional Productivity Factor:

Animal Grazing Factor:
Veg: $2.6 \mathrm{E} 7 \mathrm{kilogram}$

Milk: 1.0E8 1iter

Meat: $5.8 E 7$ kilogram

0.9

0.7

Meteorology Period of Record: 17 OCT 70 - 16 OCT $71 \quad$ Recovery: $90 \%$

SITE-SPECIFIC DATA - WATERBORNE PATHWAYS via LAKE NORMAN On CATAWBA

RIVER

Average River

Flow at Site: $2,600 \mathrm{ft}^{3} / \mathrm{s}$

Drinking Water:

Exposed Population: 720,000

Fish:

Edible Harvest: $1.8^{(\mathrm{a})} \mathrm{kg} / \mathrm{yr}$

Dilution Factor: 1

(a) Average individual consumption rate as given in FES (1976). 


\section{POPULATION DOSE-COMMITMENT ESTIMATES AND \\ AVERAGE INDIVIDUAL DOSE-COMMITMENT HISTOGRAM FOR \\ MCGUIRE I AND 2}

Dose Commitments (person-rem) from Waterborne Pathways

Total Body GI-LLI Ihyroid Bone Liver

$\begin{array}{llllll}\text { Infant } & 2.1 \mathrm{E}-01 & 2.0 \mathrm{E}-01 & 1.1 \mathrm{E}+00 & 2.1 \mathrm{E}-02 & 2.2 \mathrm{E}-01 \\ \text { Child } & 2.7 \mathrm{E}+00 & 2.8 \mathrm{E}+00 & 9.1 \mathrm{E}+00 & 2.0 \mathrm{E}+00 & 4.5 \mathrm{E}+00 \\ \text { Teen } & 1.6 \mathrm{E}+00 & 1.9 \mathrm{E}+00 & 3.1 \mathrm{E}+00 & 1.1 \mathrm{E}+00 & 2.7 \mathrm{E}+00 \\ \text { Adult } & 1.6 \mathrm{E}+00 & 1.6 \mathrm{E}+01 & 2.3 \mathrm{E}+01 & 6.6 \mathrm{E}+00 & 1.8 \mathrm{E}+01 \\ \text { TOTAL } & 2.0 \mathrm{E}+01 & 2.1 \mathrm{E}+01 & 3.6 \mathrm{E}+01 & 9.8 \mathrm{E}+00 & 2.6 \mathrm{E}+01\end{array}$

Dose Commitments (person-rem) from Airborne Pathways

Iotal Body GI-LLI Thyroid Bone Liver Lung

$\begin{array}{lllllll}\text { Infant } & 2.5 \mathrm{E}-03 & 2.2 \mathrm{E}-03 & 1.4 \mathrm{E}-02 & 2.1 \mathrm{E}-03 & 2.3 \mathrm{E}-03 & 2.4 \mathrm{E}-03 \\ \text { Child } & 2.6 \mathrm{E}-02 & 2.6 \mathrm{E}-02 & 9.9 \mathrm{E}-02 & 2.3 \mathrm{E}-02 & 2.7 \mathrm{E}-02 & 2.9 \mathrm{E}-02 \\ \text { Teen } & 1.9 \mathrm{E}-02 & 1.9 \mathrm{E}-02 & 4.9 \mathrm{E}-02 & 1.6 \mathrm{E}-02 & 1.9 \mathrm{E}-02 & 2.2 \mathrm{E}-02 \\ \text { Aduit } & 1.2 \mathrm{E}-01 & 1.1 \mathrm{E}-01 & 2.2 \mathrm{E}-01 & 9.9 \mathrm{E}-02 & 1.2 \mathrm{E}-01 & 1.2 \mathrm{E}-01 \\ \text { TOTAL } & 1.6 \mathrm{E}-01 & 1.6 \mathrm{E}-01 & 3.8 \mathrm{E}-01 & 1.4 \mathrm{E}-01 & 1.6 \mathrm{E}-01 & 1.8 \mathrm{E}-01\end{array}$

Production/Consumption factors:

Produce: 0.069 Milk: 0.41 Meat: 0.38

Fraction of Population Receiving an Average Individual Total-Body Dose Commitment from Airborne Pathways

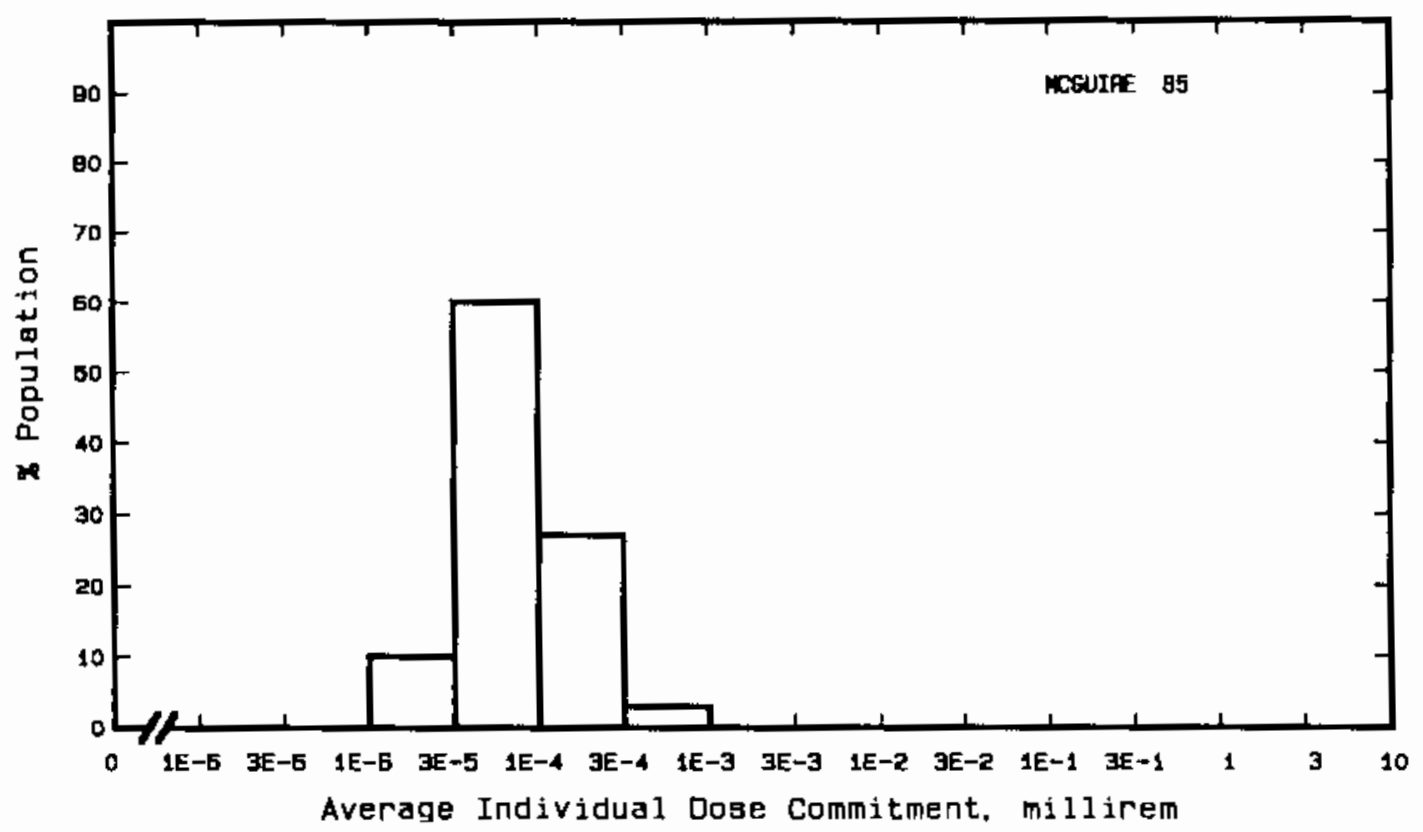


Site: MILLSTONE

Location: $\quad$ N $41.3086^{\circ}$
WATERFORD, CONNECTICUT

W $72.1681^{\circ}$

POPULATION DATA

Total Population Within 2-to-80-km Region: 2.6E6

Major Metropolitan Centers Within Region:

Center

Hartford SMSA

New Haven-West Haven SMSA

New London-Norwich SMSA

New Britain SMSA

Waterbury SMSA
Population

740,000

430,000

250,000

140,000

230,000
Location

$67 \mathrm{~km} \quad \mathrm{NW}$

$64 \mathrm{~km} \quad W$

$8 \mathrm{~km} \quad \mathrm{NNE}$

$65 \mathrm{~km} \quad \mathrm{NW}$

$78 \mathrm{~km} \quad W N W$

\section{SITE-SPECIFIC DATA - AIRBORNE PATHWAYS}

Average Annual State Production

of Crops and Animal Products

In 80-km Radius Circle

Regional Productivity Factor:

Animal Grazing Factor:
Veg: $3.2 E 7$ kilogram

Milk: 4.4E8 liter

Meat: $2.0 \mathrm{E} 7$ kilogram

0.6

0.6

Meteorology Period of Record: 1 JAN $74-31$ DEC 74 Recovery: $95 \%$

SITE-SPECIFIC DATA - WATERBORNE PATHWAYS via NIANTIC BAY

Average Dilution $\mathrm{Flow}$

from Plant: 2,600 $\mathrm{ft}^{3} / \mathrm{s}$

Fish:

Edible Harvest: $9.1 \mathrm{E} 4 \mathrm{~kg} / \mathrm{yr}$

Dilution Factor: 0.001

Invertebrates:

Edible Harvest: $9.1 \mathrm{E} 4 \mathrm{~kg} / \mathrm{yr}$

Dilution Factor: 0.002 


\begin{abstract}
POPULATION DOSE-COMMITMENT ESTIMATES ANO
AVERAGE INDIVIOUAL DOSE-COMMITMENT HISTOGRAM FOR
\end{abstract}

MILL.STONE 1 AND 2

Dose Commitments (person-rem) from Waterborne Pathways

Total Body GI-LLI Ihyroid Bone Liver

$\begin{array}{llllll}\text { Infant } & 0.0 \mathrm{E}+00 & 0.0 \mathrm{E}+00 & 0.0 \mathrm{E}+00 & 0.0 \mathrm{E}+00 & 0.0 \mathrm{E}+00 \\ \text { Child } & 4.1 \mathrm{E}-03 & 7.3 \mathrm{E}-03 & 3.1 \mathrm{E}-03 & 1.6 \mathrm{E}-02 & 1.4 \mathrm{E}-02 \\ \text { Teen } & 4.8 \mathrm{E}-03 & 1.5 \mathrm{E}-02 & 2.3 \mathrm{E}-03 & 9.2 \mathrm{E}-03 & 1.2 \mathrm{E}-02 \\ \text { Adult } & 4.4 \mathrm{E}-02 & 1.3 \mathrm{E}-01 & 1.5 \mathrm{E}-02 & 5.3 \mathrm{E}-02 & 6.8 \mathrm{E}-02 \\ \text { TOTAL. } & 5.3 \mathrm{E}-02 & 1.5 \mathrm{E}-01 & 2.0 \mathrm{E}-02 & 7.8 \mathrm{E}-02 & 9.3 \mathrm{E}-02\end{array}$

Dose Commitments (person-rem) from Airborne Pathways

Total Body GI-LLI Thyroid Bone Liver Lung

$\begin{array}{lllllll}\text { Infant } & 3.2 \mathrm{E}-03 & 3.1 \mathrm{E}-03 & 2.8 \mathrm{E}-02 & 2.2 \mathrm{E}-03 & 3.5 \mathrm{E}-03 & 3.3 \mathrm{E}-03 \\ \text { Child } & 3.7 \mathrm{E}-02 & 3.7 \mathrm{E}-02 & 1.7 \mathrm{E}-01 & 2.3 \mathrm{E}-02 & 3.8 \mathrm{E}-02 & 3.9 \mathrm{E}-02 \\ \text { Teen } & 2.6 \mathrm{E}-02 & 2.6 \mathrm{E}-02 & 8.0 \mathrm{E}-02 & 1.5 \mathrm{E}-02 & 2.6 \mathrm{E}-02 & 2.8 \mathrm{E}-02 \\ \text { Adult } & 1.5 \mathrm{E}-01 & 1.5 \mathrm{E}-01 & 3.2 \mathrm{E}-01 & 8.9 \mathrm{E}-02 & 1.5 \mathrm{E}-01 & 1.5 \mathrm{E}-01 \\ \text { TOTAL } & 2.1 \mathrm{E}-01 & 2.1 \mathrm{E}-01 & 6.0 \mathrm{E}-01 & 1.3 \mathrm{E}-01 & 2.1 \mathrm{E}-01 & 2.3 \mathrm{E}-01\end{array}$

Production/Consumption factors:

Produce: 0.038 Milk: 0.78 Meat: 0.060

Fraction of Population Receiving an Average Individual

Total-Body Dose Commitment from Airborne Pathways

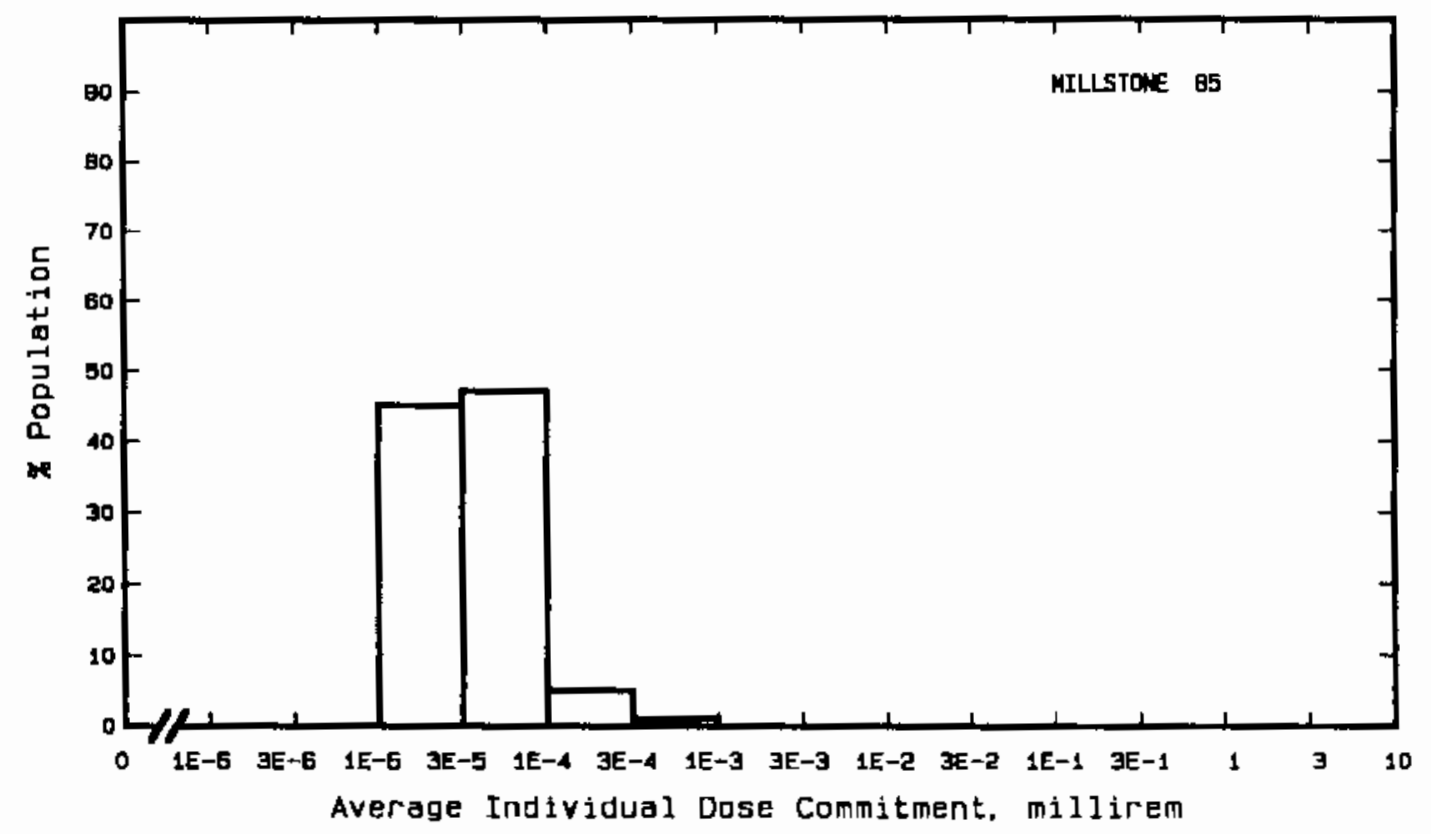


Site: MONTICELLO

MONTICELLO, MINNESOTA

Location: $\quad N 45.3333^{\circ} \quad$ W $93.8483^{\circ}$

POPULATION DATA

Total Population Within 2-to-80-km Region: 2.2E6

Major Metropolitan Centers Within Region:

Center

Population

Location

Minneapolis-St. Paul SMSA

St. Cloud SMSA

Bloomington

$2,100,000$

$60 \mathrm{~km} \quad \mathrm{SE}$

Edina

160,000

$36 \mathrm{~km} \quad \mathrm{NW}$

Richfield

83,000

$72 \mathrm{~km} \quad \mathrm{SE}$

47,000

$63 \mathrm{~km} \quad \mathrm{SE}$

39,000

$67 \mathrm{~km} \quad \mathrm{SE}$

SITE-SPECIFIC DATA - AIRBORNE PATHWAYS

Average Annual State Production

Veg: 1.2E8 kilogram

of Crops and Animal Products

Milk: 4.0E8 liter

In 80-km Radius Circle

Meat: $1.1 \mathrm{E} 8$ kilogram

Regional Productivity Factor:

Animal Grazing Factor:

1

0.5

Meteorology Period of Record: 1 JAN 74 - 31 DEC 74 Recovery: 92\%

SITE-SPECIFIC DATA - WATERBORNE PATHWAYS via MISSISSIPPI RIVER

Average River $\mathrm{Flow}$

at Site: $4,600 \mathrm{ft}^{3} / \mathrm{s}$

Drinking Water:

Exposed Population: (a)

Fish:

Edible Harvest: (a)

(a) No radionuclides released in liquid effluents reported (Tichler, et al. 1988). 
POPULATION DOSE-COMMITMENT ESTIMATES AND

AVERAGE INDIVIDUAL DOSE-COMMITMENT HISTDGRAM FOR

MONTICELLO

Dose Commitments (person-rem) from Waterborne Pathways

Total Body GI-LLI Ihyroid Bone Liver

Infant

Child

Teen

Adult

(No Waterborne Pathway Doses)

TOTAL

Dose Commitments (person-rem) from Airborne Pathways

Total Body GI-LLI Thyroid Bone Liver Lung

$\begin{array}{lllllll}\text { Infant } & 2.1 \mathrm{E}-03 & 2.0 \mathrm{E}-03 & 9.5 \mathrm{E}-02 & 1.9 \mathrm{E}-03 & 2.5 \mathrm{E}-03 & 2.1 \mathrm{E}-03 \\ \text { Child } & 2.6 \mathrm{E}-02 & 2.4 \mathrm{E}-02 & 6.5 \mathrm{E}-01 & 2.4 \mathrm{E}-02 & 2.8 \mathrm{E}-02 & 2.7 \mathrm{E}-02 \\ \text { Teen } & 1.7 \mathrm{E}-02 & 1.7 \mathrm{E}-02 & 2.6 \mathrm{E}-01 & 1.4 \mathrm{E}-02 & 1.8 \mathrm{E}-02 & 2.0 \mathrm{E}-02 \\ \text { Adult } & 9.5 \mathrm{E}-02 & 1.0 \mathrm{E}-01 & 9.3 \mathrm{E}-01 & 7.7 \mathrm{E}-02 & 9.7 \mathrm{E}-02 & 1.1 \mathrm{E}-01 \\ \text { TOTAL } & 1.4 \mathrm{E}-01 & 1.4 \mathrm{E}-01 & 1.9 \mathrm{E}+00 & 1.2 \mathrm{E}-01 & 1.5 \mathrm{E}-01 & 1.6 \mathrm{E}-01\end{array}$

Production/Consumption factors:

Produce: 0.29 Hilk: 1.4 Meat: 0.60

Fraction of Population Receiving an Average Individual

Total-Body Dose Commitment from Airborne Pathways

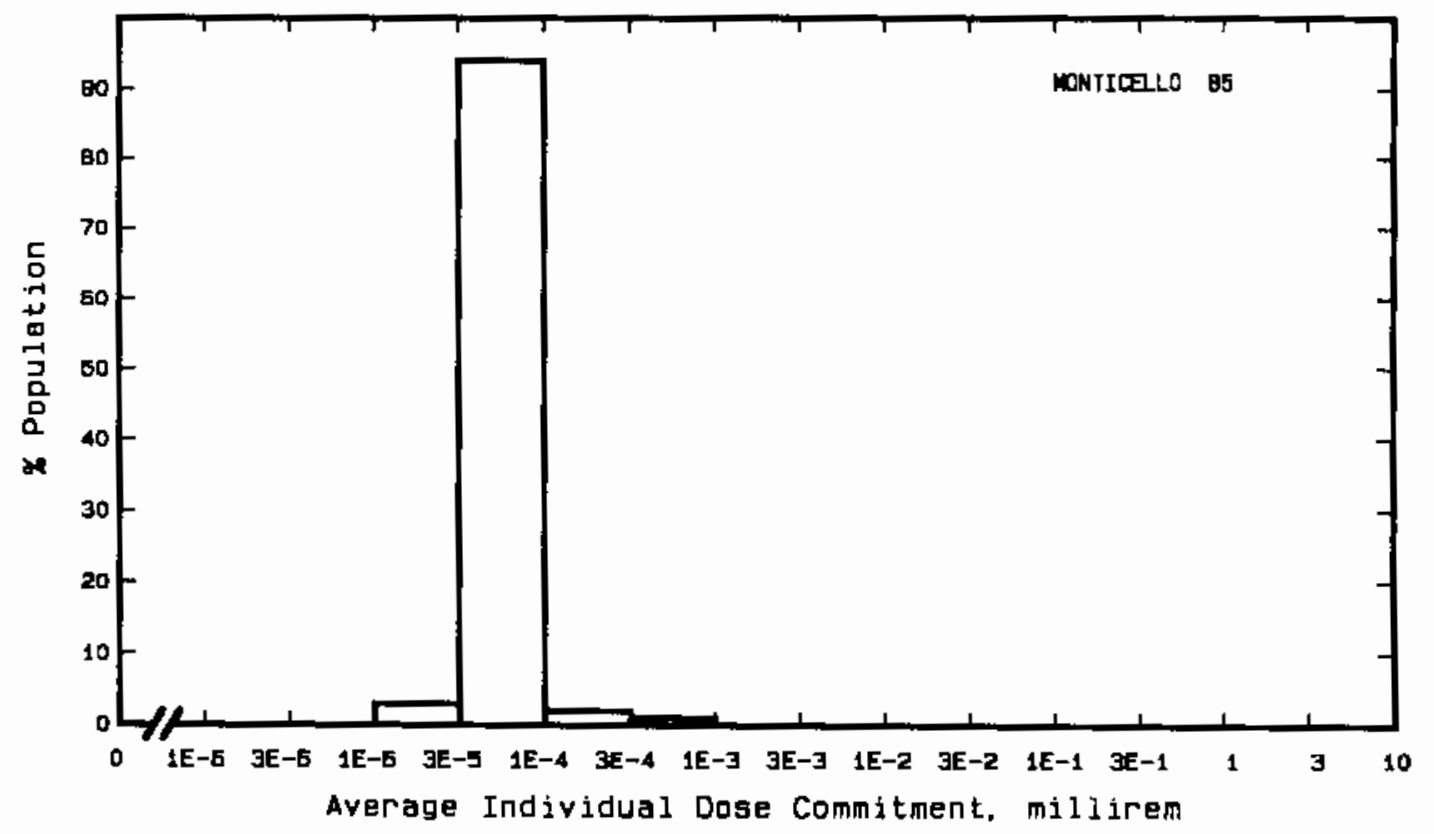


Site: NINE MILE POINT

OSWEGO, NEW YORK

Location: $\quad N 43.5222^{\circ}$ W $76.4100^{\circ}$

POPULATION DATA

Total Population Within 2-to-80-km Region: 8.5E5

Major Metropolitan Centers Within Region:

Center

Syracuse SMSA

Auburn

Watertown

Kingston

Oswego
Population

670,000

33,000

28,000

24,000

20,000
Location

$56 \mathrm{~km} \quad \mathrm{SSE}$

$67 \mathrm{~km} \quad$ SSW

$64 \mathrm{~km} \quad \mathrm{NE}$

$79 \mathrm{~km} \mathrm{~N}$

$11 \mathrm{~km} \quad \mathrm{SW}$

SITE-SPECIFIC DATA - AIRBORNE PATHWAYS

Average Annual State Production

of Crops and Animal Products

In $80-\mathrm{km}$ Radius Circle

Regional Productivity Factor:

Animal Grazing Factor:
Veg: $7.6 \mathrm{E} 7 \mathrm{kilogram}$

Milk: 7.0E8 liter

Meat: $3.3 \mathrm{E} 7 \mathrm{ki}$ logram

0.7

0.5

Meteorology Period of Record: I JAN 74 - 31 IJEC 75 Recovery: 97\%

$\begin{array}{ll} & \begin{array}{l}\text { Average Dilution Flow } \\ \text { from Plant: (a) }\end{array} \\ \text { Drinking Water: } \quad \text { Exposed Population: (a) }\end{array}$

Fish:

Edible Harvest: (a)

(a) No liquid releases reported (Tichler, et al. 1988). 


\section{POPULATION DOSE-COMMITMENT ESTIMATES AND \\ AVERAGE INDIVIDUAL DOSE-COMMITMENT HISTOGRAM FOR \\ NINE MILE POINT}

Dose Commitments (person-rem) from Waterborne Pathways

Total Body GI-LLI $\underline{\text { Thyroid Bone }}$

Infant

Child

Teen

(No Waterborne Pathway Doses)

Adult

TOTAL

Dose Commitments (person-rem) from Airborne Pathways

Total Body GI-LLI Thyroid Bone Liver Lung

$\begin{array}{lllllll}\text { Infant } & 2.6 \mathrm{E}-04 & 2.3 \mathrm{E}-04 & 1.4 \mathrm{E}-02 & 3.3 \mathrm{E}-04 & 3.9 \mathrm{E}-04 & 2.6 \mathrm{E}-04 \\ \text { Child } & 3.3 \mathrm{E}-03 & 3.1 \mathrm{E}-03 & 9.6 \mathrm{E}-02 & 4.8 \mathrm{E}-03 & 4.1 \mathrm{E}-03 & 3.3 \mathrm{E}-03 \\ \text { Teen } & 2.1 \mathrm{E}-03 & 2.1 \mathrm{E}-03 & 3.7 \mathrm{E}-02 & 2.2 \mathrm{E}-03 & 2.4 \mathrm{E}-03 & 2.2 \mathrm{E}-03 \\ \text { Adult } & 1.2 \mathrm{E}-02 & 1.2 \mathrm{E}-02 & 1.3 \mathrm{E}-01 & 1.0 \mathrm{E}-02 & 1.2 \mathrm{E}-02 & 1.2 \mathrm{E}-02 \\ \text { TOTAL } & 1.8 \mathrm{E}-02 & 1.7 \mathrm{E}-02 & 2.8 \mathrm{E}-01 & 1.8 \mathrm{E}-02 & 1.9 \mathrm{E}-02 & 1.8 \mathrm{E}-02\end{array}$

Production/Consumption factors:

Produce: $0.32 \quad$ MiTk: 4.4 Meat: 0.34

Fraction of Population Receiving an Average Individual

Total-Body Dose Commitment from Airborne Pathways

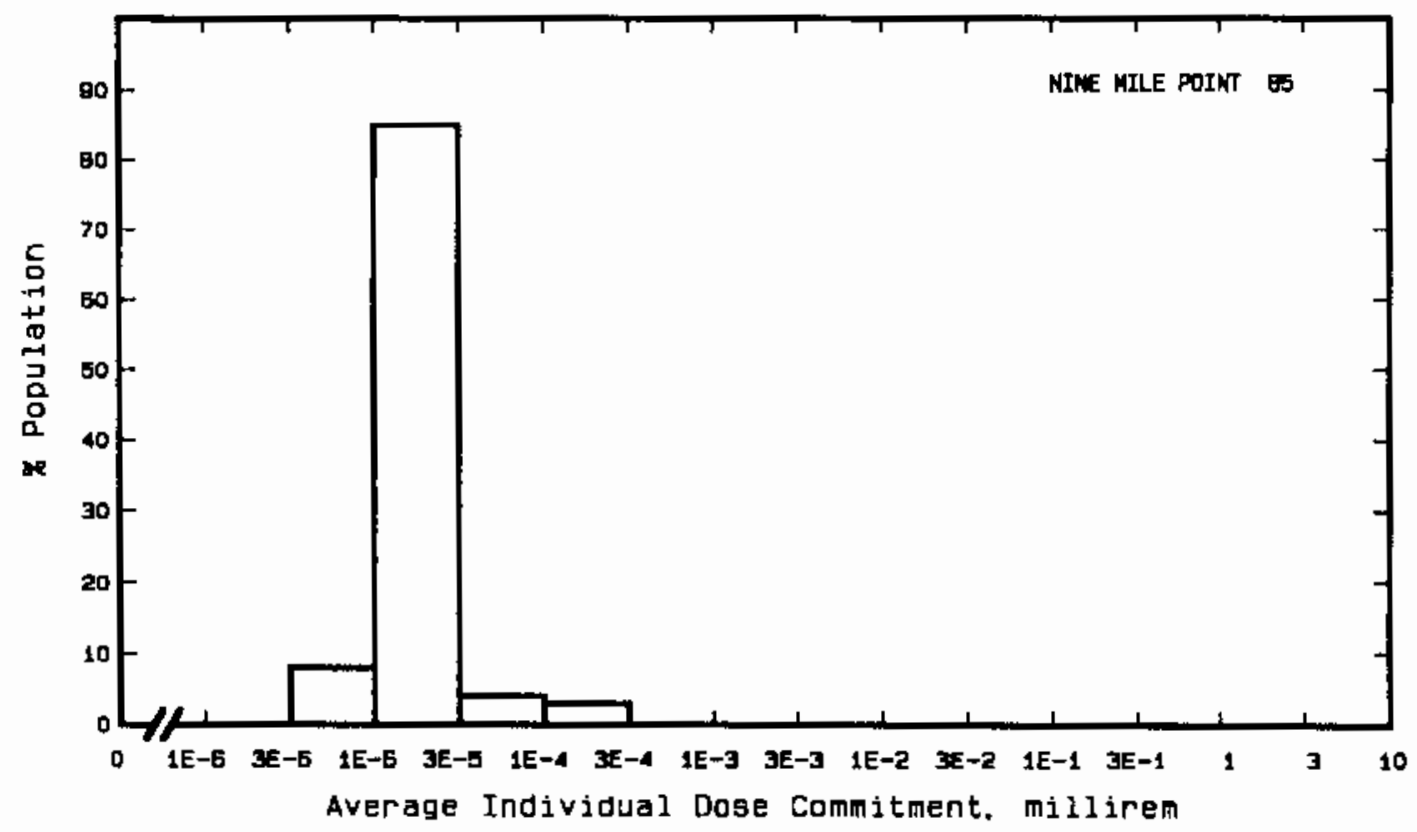


Site: NORTH ANNA

LOUISA COUNTY, VIRGINIA

Location: $\quad \mathrm{N} 38.0608^{\circ}$ W $77.7906^{\circ}$

POPULATION DATA

Total Population Within 2-to-80-km Region: 1.1E6

Major Metropolitan Centers Within Region:

Center

Richmond SMSA

Charlottesville

Fredricksburg

Culpeper

Ashland
Population

660,000

43,000

16,000

6,900

4,900
Location

$66 \mathrm{~km} \quad \mathrm{SSE}$

$63 \mathrm{~km} \quad \mathrm{~W}$

$40 \mathrm{~km} \quad \mathrm{NE}$

$54 \mathrm{~km} \quad \mathrm{NNW}$

$41 \mathrm{~km} \quad \mathrm{SE}$

\section{SITE-SPECIFIC DATA - AIRBORNE PATHWAYS}

Average Annual State Production

of Crops and Animal Products

In $80-\mathrm{km}$ Radius Circle

Regional Productivity Factor:

Animal Grazing Factor:
Veg: 3.5E7 kilogram

Milk: 1.5E8 liter

Meat: $7.4 \mathrm{E} 7$ kilogram

0.9

0.7

Meteorology Period of Record: 1 APR 74 - 31 APR 75 Recovery: $99 \%$

SITE-SPECIFIC DATA - WATERBORNE PATHWAYS via LAKE ANNA(a)

Average Dilution $\mathrm{Flow}$ from

Plant: $3,100 \mathrm{ft}^{3} / \mathrm{s}$

Drinking Water:

Exposed Population: None

Fish:

Edible Harvest: $7.3^{(\mathrm{b})} \mathrm{kg} / \mathrm{yr}$

Dilution Factor: $0.001(\mathrm{~b})$

(a) Reconcentration of radionuclides in lake accounted for (FES 1973).

(b) Average individual consumption rates as given in FES (1973) were used in lieu of catch data. 


\section{POPULATION DOSE-COMMITMENT ESTIMATES AND \\ AVERAGE INDIVIDUAL DOSE-COMMITMENT HISTOGRAM FOR}

NORTH ANNA 1 AND 2

Dose Commitments (person-rem) from Waterborne Pathways

Total Body GI-LLI $\underline{\text { Thyroid }}$

$\begin{array}{llllll}\text { Infant } & 0.0 \mathrm{E}+00 & 0.0 \mathrm{E}+00 & 0.0 \mathrm{E}+00 & 0.0 \mathrm{E}+00 & 0.0 \mathrm{E}+00 \\ \text { Child } & 1.1 \mathrm{E}+00 & 4.2 \mathrm{E}-02 & 3.7 \mathrm{E}-03 & 6.0 \mathrm{E}+00 & 6.7 \mathrm{E}+00 \\ \text { Teen } & 2.2 \mathrm{E}+00 & 8.1 \mathrm{E}-02 & 2.9 \mathrm{E}-03 & 3.6 \mathrm{E}+00 & 5.6 \mathrm{E}+00 \\ \text { Adult } & 2.4 \mathrm{E}+01 & 6.6 \mathrm{E}-01 & 2.0 \mathrm{E}-02 & 2.1 \mathrm{E}+01 & 3.3 \mathrm{E}+01 \\ \text { T0TAL } & 2.7 \mathrm{E}+01 & 7.8 \mathrm{E}-01 & 2.7 \mathrm{E}-02 & 3.0 \mathrm{E}+01 & 4.6 \mathrm{E}+01\end{array}$

Dose Commitments (person-rem) from Airborne Pathways

Total Body GI-LLI Thyroid Bone Liver Lung

$\begin{array}{lllllll}\text { Infant } & 1.3 \mathrm{E}-03 & 1.2 \mathrm{E}-03 & 2.3 \mathrm{E}-02 & 1.5 \mathrm{E}-03 & 1.7 \mathrm{E}-03 & 1.3 \mathrm{E}-03 \\ \text { Child } & 1.4 \mathrm{E}-02 & 1.4 \mathrm{E}-02 & 1.4 \mathrm{E}-01 & 1.6 \mathrm{E}-02 & 1.7 \mathrm{E}-02 & 1.5 \mathrm{E}-02 \\ \text { Teen } & 1.0 \mathrm{E}-02 & 9.9 \mathrm{E}-03 & 5.6 \mathrm{E}-02 & 1.0 \mathrm{E}-02 & 1.1 \mathrm{E}-02 & 1.2 \mathrm{E}-02 \\ \text { Adult } & 6.2 \mathrm{E}-02 & 6.0 \mathrm{E}-02 & 2.1 \mathrm{E}-01 & 6.1 \mathrm{E}-02 & 6.3 \mathrm{E}-02 & 6.6 \mathrm{E}-02 \\ \text { TOTAL } & 8.8 \mathrm{E}-02 & 8.4 \mathrm{E}-02 & 4.2 \mathrm{E}-01 & 8.9 \mathrm{E}-02 & 9.3 \mathrm{E}-02 & 9.4 \mathrm{E}-02\end{array}$

Production/Consumption factors:

Produce: $0.15 \quad$ Milk: $0.95 \quad$ Meat: 0.76

Fraction of Population Receiving an Average Individual

Total-Body Dose Commitment from Airborne Pathways

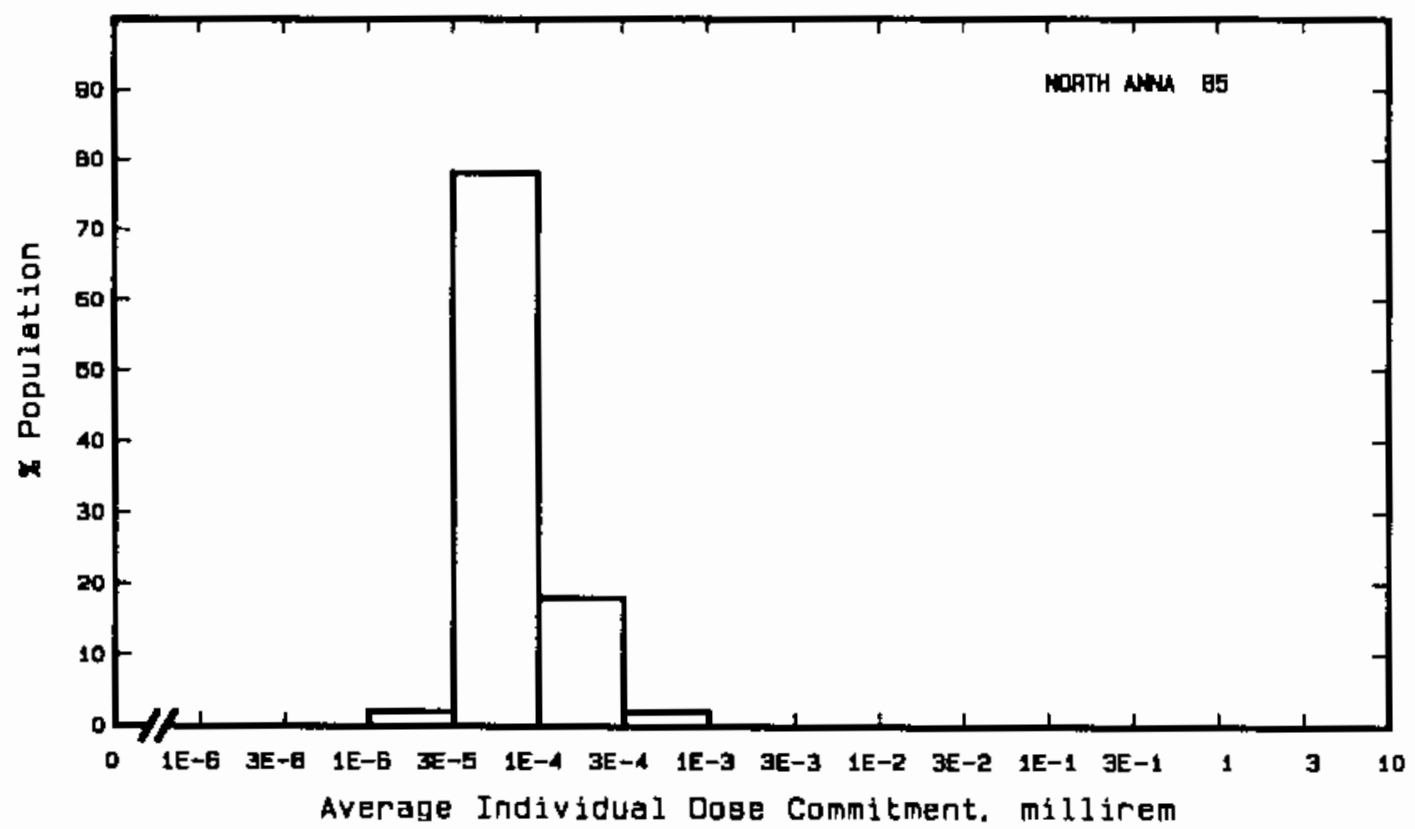


Site: OCONEE

OCONEE COUNTY, SOUTH CAROLINA

Location: N $34.7917^{\circ}$ W $82.8986^{\circ}$

POPULATION DATA

Total Population Within 2-to-80-km Region: 9.6E5

Major Metropolitan Centers Within Region:

\begin{tabular}{lrrll}
\multicolumn{1}{c}{ Center } & Population & \multicolumn{2}{c}{ Location } \\
Greenville SMSA & 600,000 & & \\
Anderson & 29,000 & $36 \mathrm{~km}$ & $\mathrm{E}$ \\
Easley & 15,000 & $39 \mathrm{~km}$ & SE \\
Greer & 12,000 & $27 \mathrm{~km}$ & $\mathrm{E}$ \\
& & $64 \mathrm{~km}$ & ENE
\end{tabular}

SITE-SPECIFIC DATA - AIRBORNE PATHWAYS

Average Annual State Production

of Crops and Animal Products

In 80-km Radius Circle

Regional Productivity Factor:

Animal Grazing Factor:
Veg: $7.4 \mathrm{E} 6$ kilogram

Milk: 5.7E7 liter

Meat: 5.0E7 kilogran

1

0.7

Meteorology Period of Record: 1 JAN 75 - 31 DEC 75 Recovery: $86 \%$

SITE-SPECIFIC DATA - WATERBORNE PATHWAYS via HARTWELL RES. ON KEOWEE

RIVER

Average River Flow

at Site: $1,100 \mathrm{ft}^{3} / \mathrm{s}$

Orinking Water:

Exposed Population: 57,000

Dilution Factor: 1

Fish:

Edible Harvest: (a) Dilution Factor: $0.01^{(b)}$

(a) No fish catch data given in FES, so generic consumption rates used (Table A-1).

(b) Ten percent of population obtain $10 \%$ of their fish diet from Hartwell Reservoir according to FES (1972). 


\section{POPULATION DOSE-COMMITMENT ESTIMATES AND \\ AVERAGE INDIVIDUAL DDSE-COMMITMENT HISTOGRAM FOR \\ OCONEE 1,2 AND 3}

Dose Commitments (person-rem) from Waterborne Pathways

Total Body GI-LII $\underline{\text { Thyroid }}$

$\begin{array}{llllll}\text { Infant } & 5.9 \mathrm{E}-02 & 6.1 \mathrm{E}-02 & 2.4 \mathrm{E}-01 & 3.0 \mathrm{E}-02 & 8.5 \mathrm{E}-02 \\ \text { Child } & 8.0 \mathrm{E}-01 & 7.7 \mathrm{E}-01 & 1.9 \mathrm{E}+00 & 1.0 \mathrm{E}+00 & 1.6 \mathrm{E}+00 \\ \text { Teen } & 5.1 \mathrm{E}-01 & 4.0 \mathrm{E}-01 & 6.5 \mathrm{E}-01 & 4.9 \mathrm{E}-01 & 9.4 \mathrm{E}-01 \\ \text { Adult } & 5.0 \mathrm{E}+00 & 3.5 \mathrm{E}+00 & 4.9 \mathrm{E}+00 & 2.9 \mathrm{E}+00 & 6.2 \mathrm{E}+00 \\ \text { TOTAL } & 6.4 \mathrm{E}+00 & 4.7 \mathrm{E}+00 & 7.7 \mathrm{E}+00 & 4.4 \mathrm{E}+00 & 8 . \mathrm{BE}+00\end{array}$

Dose Commitments (person-rem) from Airborne Pathways

Total Body GI-LII Thyroid Bone Liver Lung

$\begin{array}{lllllll}\text { Infant } & 7.2 \mathrm{E}-03 & 7.2 \mathrm{E}-03 & 8.3 \mathrm{E}-03 & 7.1 \mathrm{E}-03 & 7.2 \mathrm{E}-03 & 7.9 \mathrm{E}-03 \\ \text { Child } & 8.1 \mathrm{E}-02 & 8.1 \mathrm{E}-02 & 8.7 \mathrm{E}-02 & 7.9 \mathrm{E}-02 & 8.1 \mathrm{E}-02 & 9.0 \mathrm{E}-02 \\ \text { Teen } & 5.9 \mathrm{E}-02 & 5.9 \mathrm{E}-02 & 6.2 \mathrm{E}-02 & 5.7 \mathrm{E}-02 & 5.9 \mathrm{E}-02 & 7.1 \mathrm{E}-02 \\ \text { Adult } & 3.6 \mathrm{E}-01 & 3.6 \mathrm{E}-01 & 3.7 \mathrm{E}-01 & 3.5 \mathrm{E}-01 & 3.6 \mathrm{E}-01 & 4.0 \mathrm{E}-01 \\ \text { TOTAL } & 5.0 \mathrm{E}-01 & 5.0 \mathrm{E}-01 & 5.3 \mathrm{E}-01 & 4.9 \mathrm{E}-01 & 5.0 \mathrm{E}-01 & 5.6 \mathrm{E}-01\end{array}$

Production/Consumption factors:

Produce: $0.040 \quad$ Milk: $0.46 \quad$ Meat: 0.65

Fraction of Population Receiving an Average Individual

Total-Body Dose Commitment from Ajrborne Pathways

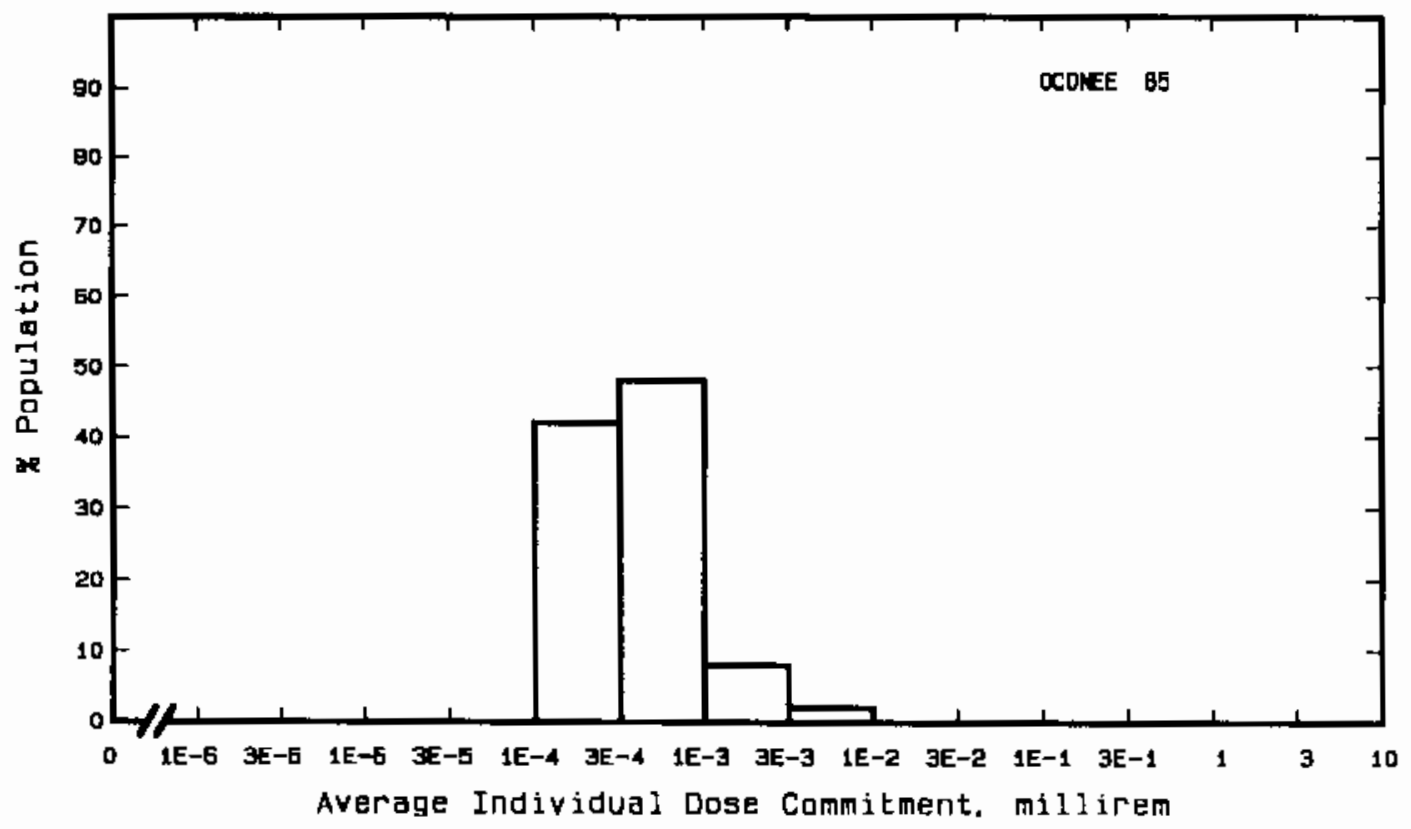


Site: OYSTER CREEK

OYSTER CFEEK, NEW JERSEY

Location: $\quad$ N $39.8142^{\circ}$ W $74.2064^{\circ}$

POPULATION DATA

Total Population Within 2-to-80-km Region: 3.6E6

Major Metropolitan Centers Within Region:

Center

New Brunswick-Sayreville SMSA

Long Branch-Asbury Park SMSA

Trenton SMSA

Atlantic City SMSA

Camden
Population

620,000

510,000

320,000

190,000

87,000
Location

$77 \mathrm{~km} \mathrm{~N}$

$57 \mathrm{~km} \quad \mathrm{NNE}$

$66 \mathrm{~km} \quad \mathrm{NW}$

$55 \mathrm{~km} \quad \mathrm{SSW}$

$79 \mathrm{~km} \quad \mathrm{~W}$

SITE-SPECIFIC DATA - AIRBORNE PATHWAYS

Average Annual State Production

of Crops and Anima1 Products

In 80-km Radius Circle

Regional Productivity Factor:

Animal Grazing Factor:

Meteorology Period of Record: 15 FEB 66 - 31 DEC 68 Recovery: $63 \%$
Veg: $7.4 E 7$ kilogram

Milk: 2.7E8 liter

Meat: $2.4 \mathrm{E} 7 \mathrm{kilogram}$

0.5

0.6

SITE-SPECIFIC DATA - WATERBORNE PATHWAYS via BARNEGAT BAY (a)

Average Dilution Flow

from Plant: (a)

Fish:

Edible Harvest: (a)

Invertebrates:

Edible Harvest: (a)

(a) No liquid releases reported (Tichler, et al. 198B). 


\section{POPULATION DOSE-COMMITMENT ESTIMATES AND \\ AVERAGE INDIVIDUAL DOSE-COMMITMENT HISTOGRAM FOR \\ OYSTER CREEK}

Dose Commitments (person-rem) from Waterborne Pathways

Total Body GI-LII Ihyroid Bone Liver

Infant

Child

Teen

(No Waterborne Pathway Doses)

Adult

TOTAL

Dose Commitments (person-rem) from Airborne Pathways

Total Body GI-LII Thyroid Bone Liver

$\begin{array}{lllllll}\text { Infant } & 1.1 \mathrm{E}+00 & 1.0 \mathrm{E}+00 & 2.6 \mathrm{E}+00 & 1.1 \mathrm{E}+00 & 1.1 \mathrm{E}+00 & 1.1 \mathrm{E}+00 \\ \text { Child } & 1.2 \mathrm{E}+01 & 1.2 \mathrm{E}+01 & 2.2 \mathrm{E}+01 & 1.2 \mathrm{E}+01 & 1.2 \mathrm{E}+01 & 1.2 \mathrm{E}+01 \\ \text { Teen } & 8.5 \mathrm{E}+00 & 8.5 \mathrm{E}+00 & 1.4 \mathrm{E}+01 & 8.5 \mathrm{E}+00 & 8.5 \mathrm{E}+00 & 9.0 \mathrm{E}+00 \\ \text { Adult } & 5.2 \mathrm{E}+01 & 5.2 \mathrm{E}+01 & 7.2 \mathrm{E}+01 & 5.2 \mathrm{E}+01 & 5.2 \mathrm{E}+01 & 5.3 \mathrm{E}+01 \\ \text { TOTAL } & 7.3 \mathrm{E}+01 & 7.3 \mathrm{E}+01 & 1.1 \mathrm{E}+02 & 7.3 \mathrm{E}+01 & 7.3 \mathrm{E}+01 & 7.5 \mathrm{E}+01\end{array}$

Production/Consumption factors:

Produce: $0.053 \quad$ Milk: 0.29 Meat: 0.042

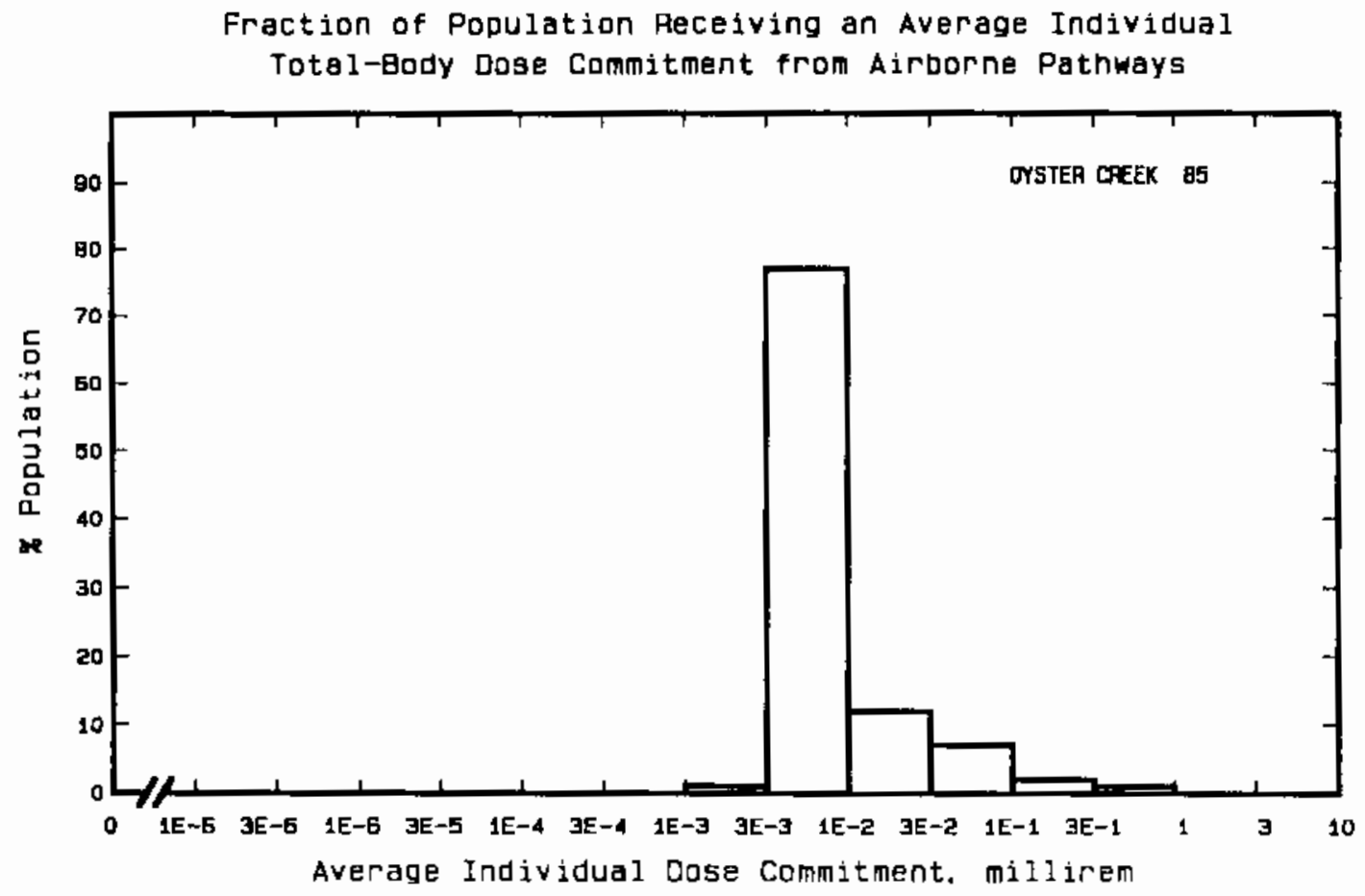


Site: PALISADES

COVERT TOWNSHIP, MICHIGAN

Location: $\quad N 42.3222^{\circ} \quad W 86.3153^{\circ}$

POPULATION DATA

Total Population Within 2-to-80-km Region: $1.0 \mathrm{E} 6$

Major Metropolitan Centers Within Region:

Center Population Location

Kalamazoo-Portage SMSA $280,000 \quad 61 \mathrm{~km} \quad \mathrm{E}$

South Bend SMSA 280,000 $72 \mathrm{~km} \quad \mathrm{~S}$

Elkhart SMSA $\quad 140,000 \quad 76 \mathrm{~km} \quad$ SSE

Holland $26,000 \quad 53 \mathrm{~km}$ NNE

Benton Harbor $\quad 15,000 \quad 25 \mathrm{~km} \quad \mathrm{SSW}$

SITE-SPECIFIC DATA - AIRBORNE PATHWAYS

Average Annual State Production

of Crops and Animal Products

In 80-km Radius Circle

Regional Productivity Factor:

Animal Grazing Factor:

Meteorology Period of Record: 1 SEP 73 - 31 AUG 74 Recovery: $67 \%$
Veg: $6.8 \mathrm{E} 7 \mathrm{kilogram}$

Milk: 2.9E8 liter

Meat: $4.5 E 7$ kilogram

0.6

0.5

SITE-SPECIFIC DATA - WATERBORNE PATHWAYS via LAKE MICHIGAN

Average Dilution Flow from P1 ant: $89 \mathrm{ft}^{3} / \mathrm{s}$

Drinking Water:

Exposed Population: 50,000

Dilution Factor: $3.5 \mathrm{E}-3(\mathrm{a})$

Fish:

Edible Harvest: (b)

Dilution Factor: 0.001

(a) Drinking water dilution factor estimated by averaging dilution factor derived from FES (1972) suitably weighted for population.

(b) Average individual consumption rate of $20 \mathrm{~g} / \mathrm{d}$ as given in FES used in lieu of catch data. 
POPULATION DOSE-COMMITMENT ESTIMATES AND

AVERAGE INDIVIOUAL DOSE-COMMITMENT HISTOGRAM FOR

\section{PALISADES}

Dose Commitments (person-rem) from Waterborne Pathways Total Body GI-LII Thyroid Bone Liver

$\begin{array}{llllll}\text { Infant } & 7.2 \mathrm{E}-04 & 7.1 \mathrm{E}-04 & 7.7 \mathrm{E}-04 & 6.4 \mathrm{E}-05 & 7.8 \mathrm{E}-04 \\ \text { Child } & 1.8 \mathrm{E}-02 & 8.9 \mathrm{E}-03 & \mathrm{~B} .9 \mathrm{E}-03 & 5.3 \mathrm{E}-02 & 6.6 \mathrm{E}-02 \\ \text { Teen } & 2.2 \mathrm{E}-02 & 4.5 \mathrm{E}-03 & 3.6 \mathrm{E}-03 & 3.1 \mathrm{E}-02 & 5.1 \mathrm{E}-02 \\ \text { Adu1t } & 2.3 \mathrm{E}-01 & 3.8 \mathrm{E}-02 & 3.0 \mathrm{E}-02 & 1.8 \mathrm{E}-01 & 3.1 \mathrm{E}-01 \\ \text { TOTAL } & 2.7 \mathrm{E}-01 & 5.2 \mathrm{E}-02 & 4.4 \mathrm{E}-02 & 2.7 \mathrm{E}-01 & 4.3 \mathrm{E}-01\end{array}$

Dose Commitments (person-rem) from Airborne Pathways

Total Body GI-LII Thyroid Bone Liver lung

$\begin{array}{lllllll}\text { Infant } & 5.2 \mathrm{E}-04 & 5.1 \mathrm{E}-04 & 9.0 \mathrm{E}-03 & 5.2 \mathrm{E}-04 & 5.3 \mathrm{E}-04 & 5.5 \mathrm{E}-04 \\ \text { Child } & 5.8 \mathrm{E}-03 & 5.7 \mathrm{E}-03 & 5.7 \mathrm{E}-02 & 5.7 \mathrm{E}-03 & 5.8 \mathrm{E}-03 & 6.3 \mathrm{E}-03 \\ \text { Teen } & 4.2 \mathrm{E}-03 & 4.1 \mathrm{E}-03 & 2.4 \mathrm{E}-02 & 4.1 \mathrm{E}-03 & 4.2 \mathrm{E}-03 & 5.0 \mathrm{E}-03 \\ \text { Adult } & 2.5 \mathrm{E}-02 & 2.5 \mathrm{E}-02 & 9.1 \mathrm{E}-02 & 2.5 \mathrm{E}-02 & 2.5 \mathrm{E}-02 & 2.8 \mathrm{E}-02 \\ \text { TOTAL } & 3.5 \mathrm{E}-02 & 3.5 \mathrm{E}-02 & 1.8 \mathrm{E}-01 & 3.5 \mathrm{E}-02 & 3.6 \mathrm{E}-02 & 3.9 \mathrm{E}-02\end{array}$

Production/Consumption factors:

Produce: $0.20 \quad$ Milk: $1.3 \quad$ Meat: 0.32

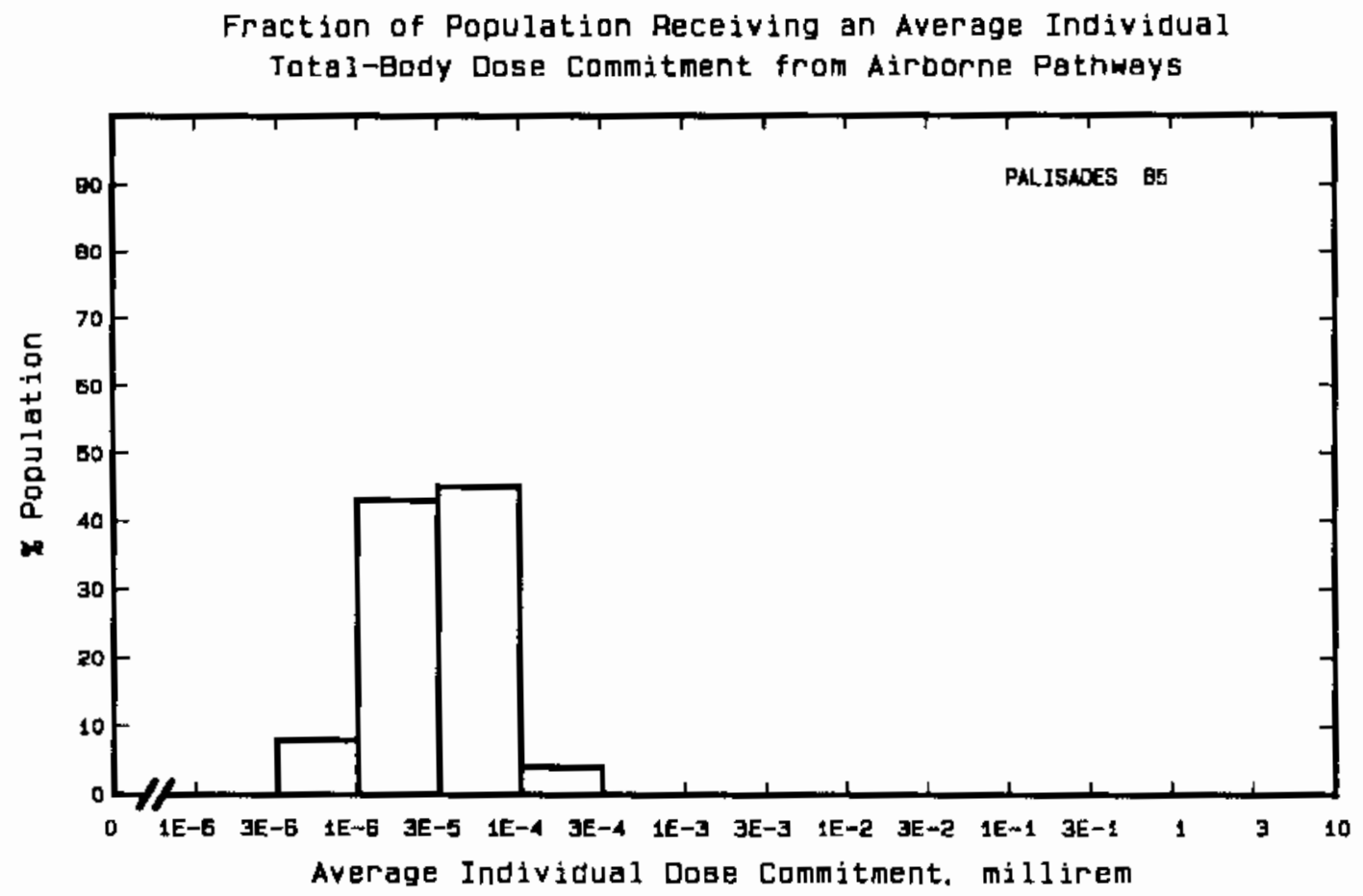


Site: PALO VERDE

WINTERSBURG, ARIZONA

Location: $\quad$ N $33.4200^{\circ} \quad$ W $112.863^{\circ}$

POPULATION DATA

Total Population Within 2-to-80-km Region: J.1E6

Major Metropolitan Centers Within Region:

\begin{tabular}{lcccc}
\multicolumn{1}{c}{ Center } & Population & \multicolumn{2}{c}{ Location } \\
Phoenix MSA & $1,800,000$ & $64 \mathrm{~km}$ & $\mathrm{E}$ \\
Avondale & 8,000 & $49 \mathrm{~km}$ & $\mathrm{E}$
\end{tabular}

SITE-SPECIFIC DATA - AIRBORNE PATHWAYS

Average Annual State Production

Veg: $1.2 \mathrm{E} 7 \mathrm{kilogram}$

of Crops and Animal Products

Milk: 2.3E7 liter

In 80-km Radius Circle

Meat: $2.1 \mathrm{E} 7 \mathrm{kilogram}$

Regional Productivity Factor:

Animal Grazing Factor:

0.9

$0.1 \mathrm{Mi} \mathrm{k}^{(\mathrm{a})}$

0.9 Beef

Meteorology Period of Record: 13 AUG $73-8$ AUG 78 Recovery: $93 \%$

SITE-SPECIFIC DATA - WATERBORNE PATHWAYS NONe

Average River Flow

at Site: (b)

Drinking Water:

Exposed Population: (b)

Fish:

Edible Harvest:

(b)

(a) Milk cows fed from dry lot $90 \%$ of time (FES 1975).

(b) No waterborne pathways 


\section{POPULATION DOSE-COMMITMENT ESTIMATES AND AVERAGE INDIVIDUAL DDSE-COMMITMENT HISTOGRAM FOR \\ PALO VERDE 1,2 , AND 3}

Infant

Dose Commitments (person-rem) from Waterborne Pathways

Child

Teen

Adult

$$
\text { Total Body GI-LLI Thyroid Bone Liver }
$$

TOTAL.

$$
\text { (No Waterborne Pathway Doses) }
$$

Dose Commitments (person-rem) from Airborne Pathways

Total Body GI-LLI Thyroid Bone Liver lung

$\begin{array}{lllllll}\text { Infant } & 4.5 \mathrm{E}-05 & 4.5 \mathrm{E}-05 & 9.1 \mathrm{E}-05 & 4.0 \mathrm{E}-05 & 4.5 \mathrm{E}-05 & 4.8 \mathrm{E}-05 \\ \text { ChiTd } & 5.3 \mathrm{E}-04 & 5.3 \mathrm{E}-04 & 1.2 \mathrm{E}-03 & 4.5 \mathrm{E}-04 & 5.3 \mathrm{E}-04 & 5.8 \mathrm{E}-04 \\ \text { Teen } & 3.9 \mathrm{E}-04 & 3.9 \mathrm{E}-04 & 7.2 \mathrm{E}-04 & 3.3 \mathrm{E}-04 & 3.9 \mathrm{E}-04 & 4.5 \mathrm{E}-04 \\ \text { Adult } & 2.3 \mathrm{E}-03 & 2.3 \mathrm{E}-03 & 3.9 \mathrm{E}-03 & 2.0 \mathrm{E}-03 & 2.3 \mathrm{E}-03 & 2.5 \mathrm{E}-03 \\ \text { TOTAL } & 3.3 \mathrm{E}-03 & 3.3 \mathrm{E}-03 & 5.8 \mathrm{E}-03 & 2.8 \mathrm{E}-03 & 3.3 \mathrm{E}-03 & 3.6 \mathrm{E}-03\end{array}$

Production/Consumption factors:

Produce: 0.048 Milk: 0.14 Meat: 0.21

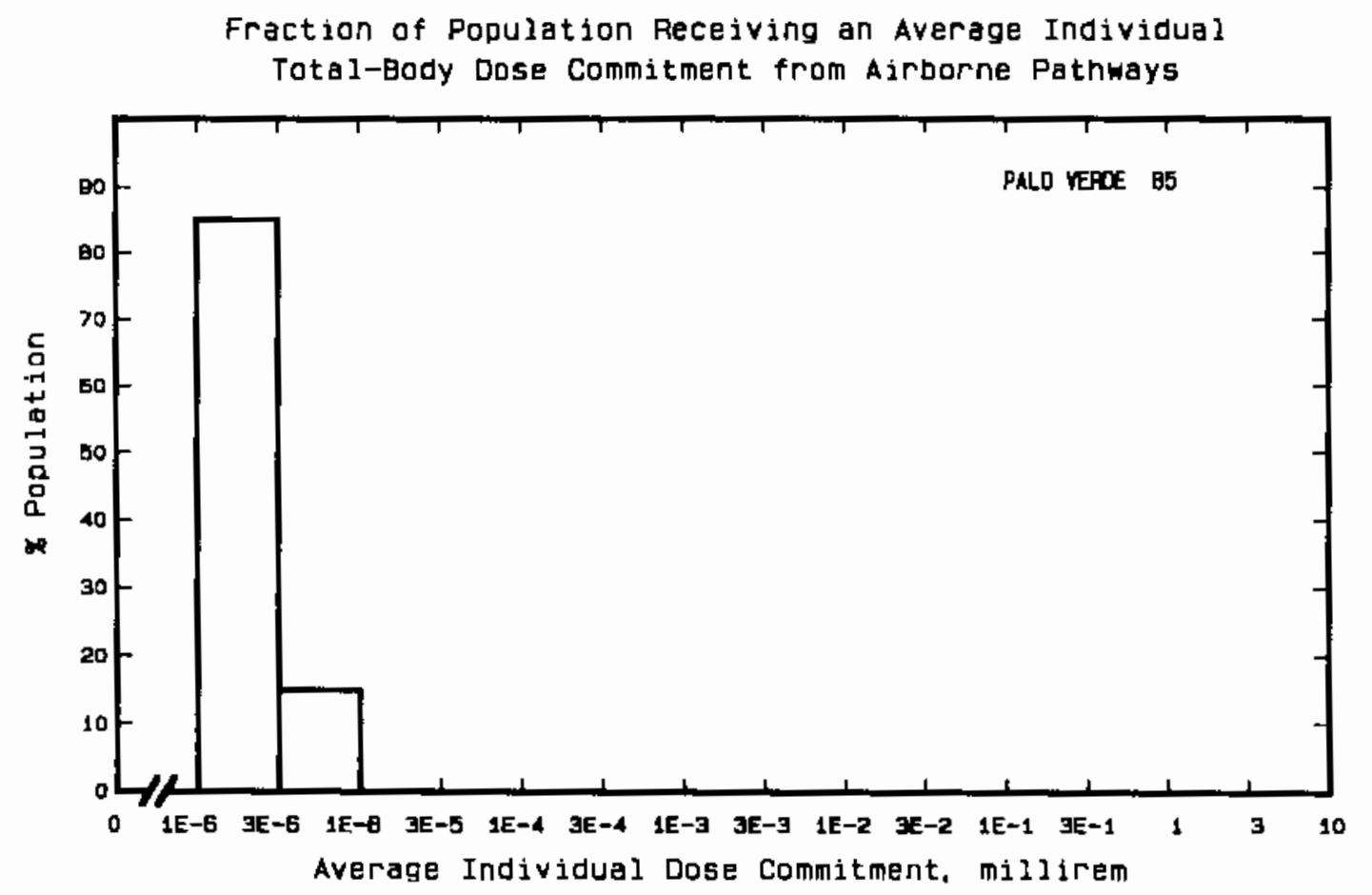


Site: PEACH BOTTOM

YORK COUNTY, PENNSYLVANIA

Location: $\quad$ N $39.7589^{\circ}$ W $76.2692^{\circ}$

POPULATION DATA

Total Population Within 2-to-80-km Region: $4.3 \mathrm{E} 6$

Major Metropolitan Centers Within Region:

Center

Baltimore SMSA

Harrisburg SMSA

WiImington SMSA

York SMSA

Lancaster SMSA
Population

$2,200,000$

450,000

400,000

380,000

360,000
Location

$60 \mathrm{~km} \quad$ SSW

$77 \mathrm{~km} \quad \mathrm{NNW}$

$62 \mathrm{~km} \quad \mathrm{E}$

$45 \mathrm{~km} \quad \mathrm{NW}$

$31 \mathrm{~km} \mathrm{~N}$

SITE-SPECIFIC DATA - AIRBORNE PATHWAYS

Average Annual State Production

Df Crops and Animal Products

In 80-km Radius Circle

Regional Productivity Factor:

Animal Grazing Factor:
Veg: $5.3 E 7$ kilogram

Mi1k: 5.3E8 liter

Meat: $5.4 E 7$ kilogram

0.95

0.6

Meteorology Period of Record: 1 AUG 67 - 31 JUL 71 Recovery: $72 \%$

SITE-SPECIFIC DATA - WATERBORNE PATHWAYS via SUSQUEHANNA RIVER

Average River Flow

at Site: $36,000 \mathrm{ft}^{3} / \mathrm{s}$

Drinking Water:

Exposed Population: 2.2E6

Dilution Factor: 1

Fish:

Edible Harvest: (a)

Dilution Factor: 0.001 (b)

(a) No fish catch data given in FES (1974), thus generic consumption rates used (Table A-1).

(b) One percent of people obtain $10 \%$ of their fish diet from river downstream from plant according to FES. 


\section{POPULATION DOSE-COMMITMENT ESTIMATES AND \\ AVERAGE INDIVIDUAL DOSE-COMMITMENT HISTOGRAM FOR}

PEACH BOTTOM 2 AND 3

Dose Commitments (person-rem) from Waterborne Pathways

Total Body $\underline{\text { GI-LII }}$ Thyroid Bone

$\begin{array}{llllll}\text { Infant } & 9.6 \mathrm{E}-03 & 5.9 \mathrm{E}-03 & 2.7 \mathrm{E}-01 & 3.9 \mathrm{E}-02 & 5.6 \mathrm{E}-02 \\ \text { Child } & 1.5 \mathrm{E}-01 & 5.8 \mathrm{E}-02 & 1.9 \mathrm{E}+00 & 4.3 \mathrm{E}-01 & 5.2 \mathrm{E}-01 \\ \text { Teen } & 9.4 \mathrm{E}-02 & 3.7 \mathrm{E}-02 & 5.8 \mathrm{E}-01 & 1.2 \mathrm{E}-01 & 2.0 \mathrm{E}-01 \\ \text { Adult } & 1.0 \mathrm{E}+00 & 3.3 \mathrm{E}-01 & 4.1 \mathrm{E}+00 & 7.6 \mathrm{E}-01 & 1.3 \mathrm{E}+00 \\ \text { TOTAL } & 1.2 \mathrm{E}+00 & 4.3 \mathrm{E}-01 & 6.9 \mathrm{E}+00 & 1.4 \mathrm{E}+00 & 2.1 \mathrm{E}+00\end{array}$

Dose Commitments (person-rem) from Airborne Pathways

Total Body GI-LII Ihyroid Bone Liver Lung

$\begin{array}{lllllll}\text { Infant } & 2.1 \mathrm{E}-01 & 2.1 \mathrm{E}-01 & 3.2 \mathrm{E}-01 & 2.1 \mathrm{E}-01 & 2.1 \mathrm{E}-01 & 2.3 \mathrm{E}-01 \\ \text { Child } & 2.4 \mathrm{E}+00 & 2.4 \mathrm{E}+00 & 3.0 \mathrm{E}+00 & 2.4 \mathrm{E}+00 & 2.4 \mathrm{E}+00 & 2.6 \mathrm{E}+00 \\ \text { Teen } & 1.7 \mathrm{E}+00 & 1.7 \mathrm{E}-00 & 2.0 \mathrm{E}+00 & 1.7 \mathrm{E}+00 & 1.7 \mathrm{E}+00 & 2.0 \mathrm{E}+00 \\ \text { Adult } & 1.0 \mathrm{E}+01 & 1.0 \mathrm{E}+01 & 1.1 \mathrm{E}+01 & 1.0 \mathrm{E}+01 & 1.0 \mathrm{E}+01 & 1.1 \mathrm{E}+01 \\ \text { TOTAL } & 1.5 \mathrm{E}+01 & 1.5 \mathrm{E}+01 & 1.7 \mathrm{E}+01 & 1.5 \mathrm{E}+01 & 1.5 \mathrm{E}+01 & 1.6 \mathrm{E}+01\end{array}$

Production/Consumption factors:

Produce: 0.061 Milk: 0.89 Meat: 0.15

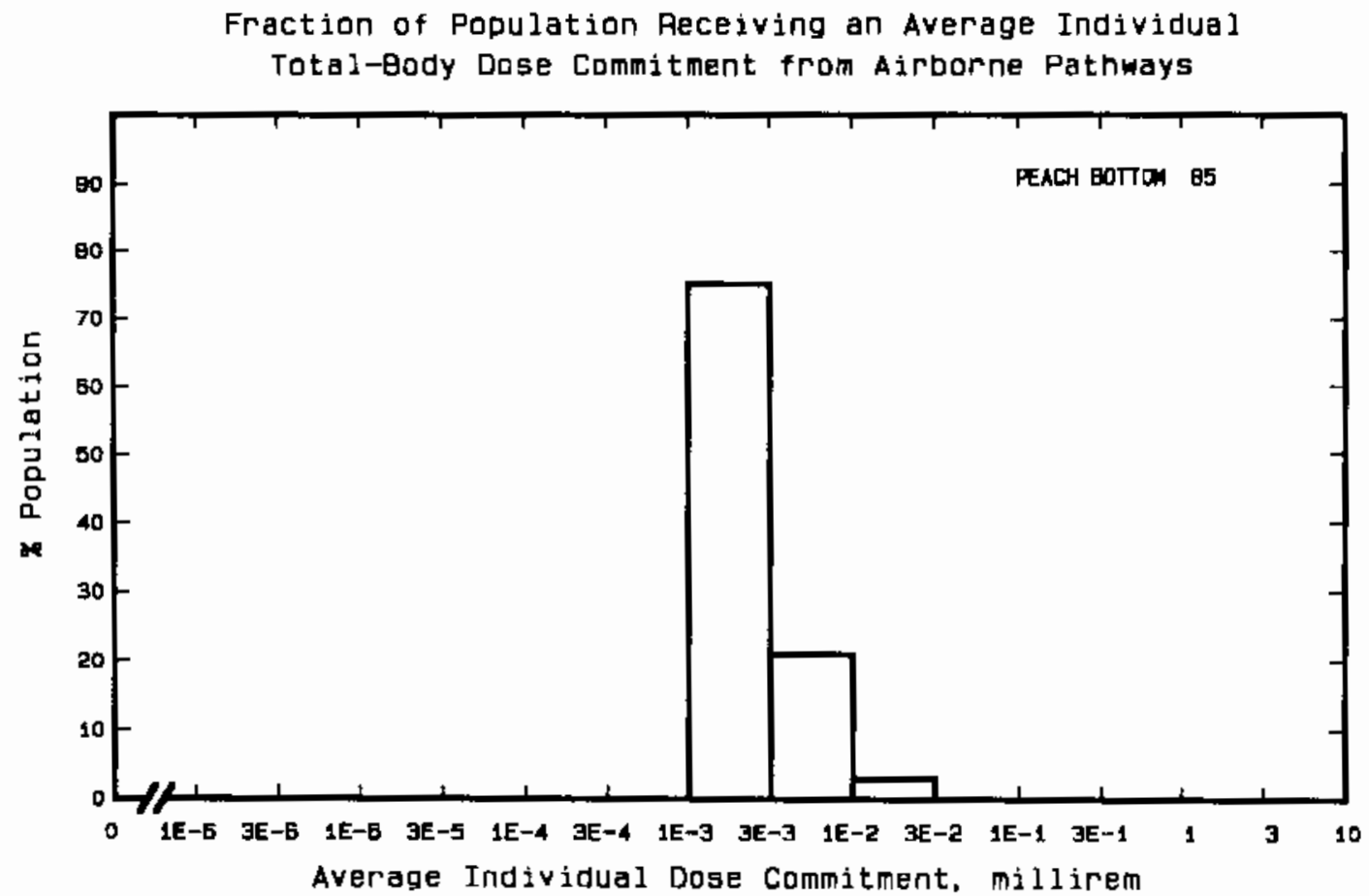


Site: PILGRIM

PLYMOUTH, MASSACHUSETTS

Location: $\quad N 41.9444^{\circ}$ W $70.5794^{\circ}$

POPULATION DATA

Total Population Within 2-to-80-km Region: 4.3E6

Major Metropolitan Centers Within Region:

Center Population Location

Boston SMSA

$2,800,000$

$61 \mathrm{~km} \quad \mathrm{NW}$

Providence-Warwick-Pawtucket SMSA

New Bedford SMSA

820,000

$70 \mathrm{~km} \quad W$

Brockton SMSA

170,001

$45 \mathrm{~km} \quad \mathrm{SSW}$

Fall River SMSA

$40 \mathrm{~km} \quad \mathrm{WNW}$

$150,000 \quad 55 \mathrm{~km} \quad \mathrm{SW}$

SITE-SPECIFIC DATA - AIRBORNE PATHWAYS

Average Annual State Production

of Crops and Animal Products

In $80-\mathrm{km}$ Radius Circle

Regional Productivity Factor:

Animal Grazing Factor:
Veg: 2.0E7 kilogram

Milk: 2.6E8 liter

Meat: $1.6 \mathrm{E} 7 \mathrm{kilogram}$

0.3

0.6

Meteorology Period of Record: 1 MAY 74 - 30 APR 75 Recovery: 93\%

Fish:

Invertebrates: via CAPE COD BAY

Average Dilution Flow

from Plant: $21 \mathrm{ft}^{3} / \mathrm{s}$

Edible Harvest: $2.6 \mathrm{E} 4 \mathrm{~kg} / \mathrm{yr}$

Dilution Factor: 0.001

Edible Harvest: $3.1 \mathrm{E} 4 \mathrm{~kg} / \mathrm{yr}$

Dilution Factor: 0.002 


\section{POPULATION DOSE-COMMITMENT ESTIMATES AND}

AVERAGE INDIVIDUAL DOSE-COMMITMENT HISTOGRAM FOR

\section{PILGRIM}

Dose Commitments (person-rem) from Waterborne Pathways

Iotal Body GI-LII Thyroid Bone Liver

$\begin{array}{llllll}\text { Infant } & 0.0 \mathrm{E}+00 & 0.0 \mathrm{E}+00 & 0.0 \mathrm{E}+00 & 0.0 \mathrm{E}+00 & 0.0 \mathrm{E}+00 \\ \text { Child } & 2.6 \mathrm{E}-03 & 4.5 \mathrm{E}-03 & 5.6 \mathrm{E}-05 & 1.5 \mathrm{E}-03 & 1.9 \mathrm{E}-03 \\ \text { Teen } & 1.9 \mathrm{E}-03 & 9.4 \mathrm{E}-03 & 3.9 \mathrm{E}-05 & 8.6 \mathrm{E}-04 & 1.5 \mathrm{E}-03 \\ \text { Adult } & 1.2 \mathrm{E}-02 & 8.3 \mathrm{E}-02 & 2.6 \mathrm{E}-04 & 5.0 \mathrm{E}-03 & 9.2 \mathrm{E}-03 \\ \text { TOTAL } & 1.6 \mathrm{E}-02 & 9.7 \mathrm{E}-02 & 3.5 \mathrm{E}-04 & 7.4 \mathrm{E}-03 & 1.3 \mathrm{E}-02\end{array}$

Dose Commitments (person-rem) from Airborne Pathways Total Body GI-LII Thyroid Bone Liver $\underline{\text { Lung }}$

$\begin{array}{lllllll}\text { Infant } & 7.8 \mathrm{E}-03 & 7.7 \mathrm{E}-03 & 3.1 \mathrm{E}-02 & 7.8 \mathrm{E}-03 & 7.9 \mathrm{E}-03 & 8.0 \mathrm{E}-03 \\ \text { Child } & 8.6 \mathrm{E}-02 & 8.6 \mathrm{E}-02 & 2.4 \mathrm{E}-01 & 8.7 \mathrm{E}-02 & 8.7 \mathrm{E}-02 & 9.0 \mathrm{E}-02 \\ \text { Teen } & 6.3 \mathrm{E}-02 & 6.3 \mathrm{E}-02 & 1.4 \mathrm{E}-01 & 6.3 \mathrm{E}-02 & 6.3 \mathrm{E}-02 & 6.8 \mathrm{E}-02 \\ \text { Adult } & 3.8 \mathrm{E}-01 & 3.8 \mathrm{E}-01 & 6.7 \mathrm{E}-01 & 3.8 \mathrm{E}-01 & 3.8 \mathrm{E}-01 & 4.0 \mathrm{E}-01 \\ \text { TOTAL } & 5.4 \mathrm{E}-01 & 5.4 \mathrm{E}-01 & 1.1 \mathrm{E}+00 & 5.4 \mathrm{E}-01 & 5.4 \mathrm{E}-01 & 5.6 \mathrm{E}-01\end{array}$

Production/Consumption factors:

Produce: 0.0071 Milk: $0.13 \quad$ Meat: 0.014

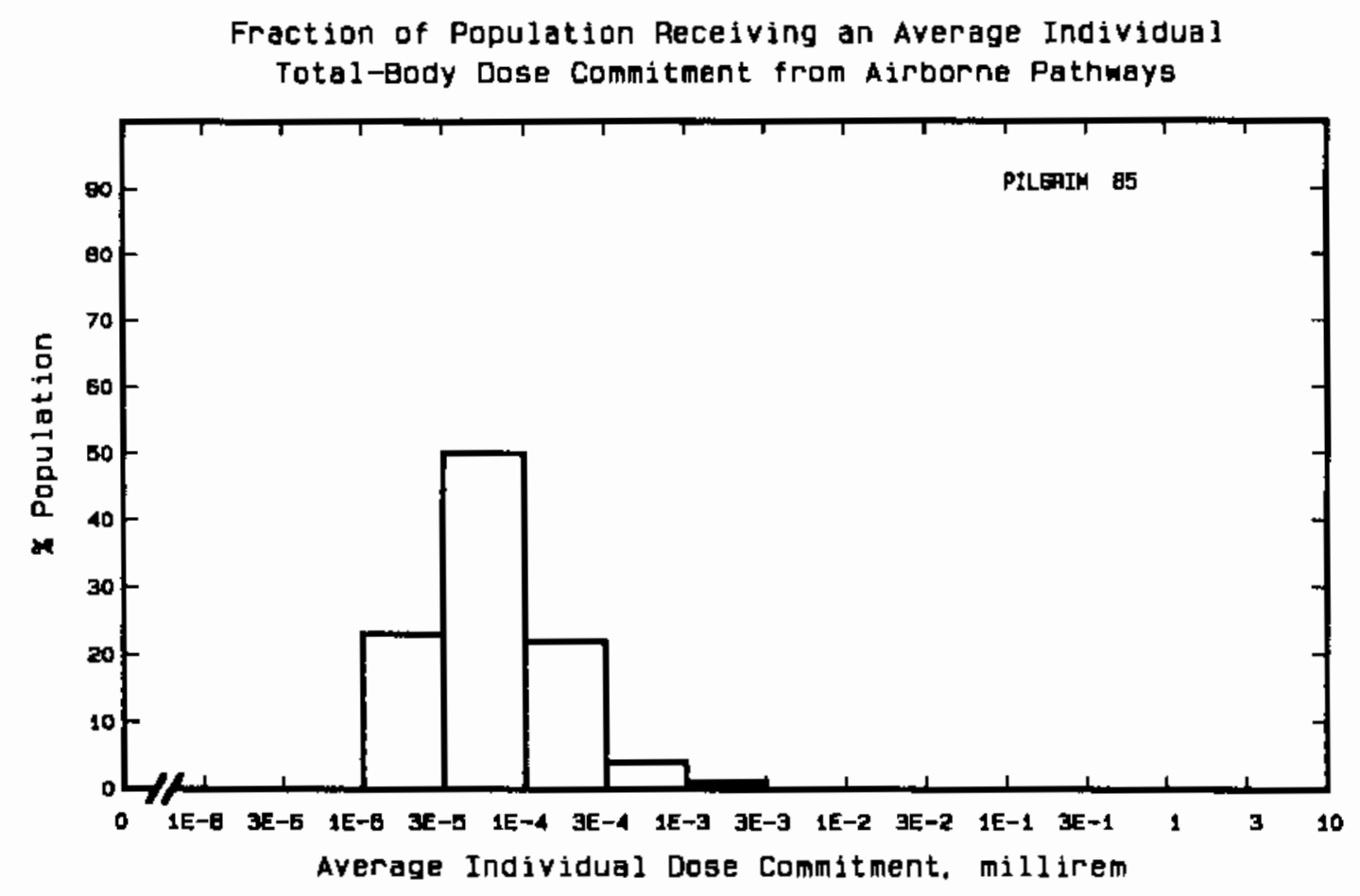


Site: POINT BEACH

MANITOWOC COUNTY, WISCONSIN

Location: $\quad$ N $44.2808^{\circ} \quad$ W $87.5361^{\circ}$

POPULATION DATA

Total Population Within 2-to-80-km Region: 6.2E5

Major Metropolitan Centers Within Region:

$\quad$ Center
Greenbay SMSA
Appleton
Sheboygan
Manitowoc
Neenah

Population

Location

Greenbay SMSA

180,000

60,000

48,000

33,000

22,000

$47 \mathrm{~km} \quad \mathrm{NW}$

$72 \mathrm{~km} \quad \mathrm{~W}$

$60 \mathrm{~km} \quad \mathrm{SSW}$

$24 \mathrm{~km} \quad \mathrm{SSW}$

$75 \mathrm{~km} \quad W$

SITE-SPECIFIC DATA - AIRBORNE PATHWAYS

Average Annual State Production

of Crops and Animal Products

In $80-\mathrm{km}$ Radius Circle

Regional Productivity Factor:

Animal Grazing Factor:
Veg: $7.2 \mathrm{E} 7 \mathrm{kilogram}$

Milk: $1.2 \mathrm{Eg}$ liter

Meat: $1.0 \mathrm{E} 8$ kilogram

0.5

0.5

Meteorology Period of Record: 19 APR $67-18$ APR 69 Recovery: 83\%

SITE-SPECIFIC DATA - WATERBORNE PATHWAYS via. LAKE MICHIGAN

Average Dilution Flow

from Plant: $710 \mathrm{ft}^{3} / \mathrm{s}$

Drinking Water:

Exposed Population: 260,000

Dilution Factor: $2.6 \mathrm{E}-3(\mathrm{a})$

Fish:

Edible Harvest: $6.7 E 4(\mathrm{~b}) \mathrm{kg} / \mathrm{yr}$

Dilution Factor: 0.013 (c)

(a) Drinking water dilution factor estimated by averaging dilution factor derived from FES (1972), suitably weighted for population.

(b) Includes both sport and commercial fish catch from FES.

(c) Dilution factor estimated by averaging sport and commercial dilution factor derived from FES. 
POPULATION DOSE-COMMITMENT ESTIMATES AND

AVERAGE INDIVIDUAL DOSE-COMMITMENT HISTOGRAM FOR

POINT BEACH 1 AND 2

Dose Commitments (person-rem) from Waterborne Pathways

Total Body $\underline{\text { GI-LII }}$ Thrroid $\underline{\text { Bone }}$ Liver

$\begin{array}{llllll}\text { Infant } & 7.3 \mathrm{E}-04 & 7.0 \mathrm{E}-04 & 4.6 \mathrm{E}-03 & 2.6 \mathrm{E}-04 & 9.3 \mathrm{E}-04 \\ \text { Child } & 9.8 \mathrm{E}-03 & 8.5 \mathrm{E}-03 & 3.6 \mathrm{E}-02 & 1.0 \mathrm{E}-02 & 1.8 \mathrm{E}-02 \\ \text { Teen } & 6.0 \mathrm{E}-03 & 4.2 \mathrm{E}-03 & 1.1 \mathrm{E}-02 & 5.1 \mathrm{E}-03 & 1.0 \mathrm{E}-02 \\ \text { Adult } & 5.8 \mathrm{E}-02 & 3.6 \mathrm{E}-02 & 8.4 \mathrm{E}-02 & 3.1 \mathrm{E}-02 & 7.0 \mathrm{E}-02 \\ \text { TOTAL } & 7.5 \mathrm{E}-02 & 5.0 \mathrm{E}-02 & 1.4 \mathrm{E}-01 & 4.6 \mathrm{E}-02 & 9.9 \mathrm{E}-02\end{array}$

Dose Commitments (person-rem) from Airborne Pathways

Total Body GI-LII Thyroid Bone Liver Lung

$\begin{array}{lllllll}\text { Infant } & 2.5 \mathrm{E}-04 & 2.3 \mathrm{E}-04 & 1.2 \mathrm{E}-03 & 2.2 \mathrm{E}-04 & 4.5 \mathrm{E}-04 & 2.5 \mathrm{E}-04 \\ \text { Child } & 3.8 \mathrm{E}-03 & 3.4 \mathrm{E}-03 & 1.0 \mathrm{E}-02 & 2.2 \mathrm{E}-03 & 5.4 \mathrm{E}-03 & 3.7 \mathrm{E}-03 \\ \text { Teen } & 2.4 \mathrm{E}-03 & 2.1 \mathrm{E}-03 & 4.8 \mathrm{E}-03 & 8.8 \mathrm{E}-04 & 3.0 \mathrm{E}-03 & 2.3 \mathrm{E}-03 \\ \text { Adult } & 1.4 \mathrm{E}-02 & 1.2 \mathrm{E}-02 & 2.1 \mathrm{E}-02 & 3.7 \mathrm{E}-03 & 1.4 \mathrm{E}-02 & 1.2 \mathrm{E}-02 \\ \text { TOTAL } & 2.0 \mathrm{E}-02 & 1.8 \mathrm{E}-02 & 3.7 \mathrm{E}-02 & 7.0 \mathrm{E}-03 & 2.3 \mathrm{E}-02 & 1.8 \mathrm{E}-02\end{array}$

Production/Consumption factors:

Produce: $0.30 \quad$ Milk: $7.2 \quad$ Meat: 1.0

Fraction of Population Receiving an Average Individual

Total-Bady Dose Commitment from Airborne Pathways

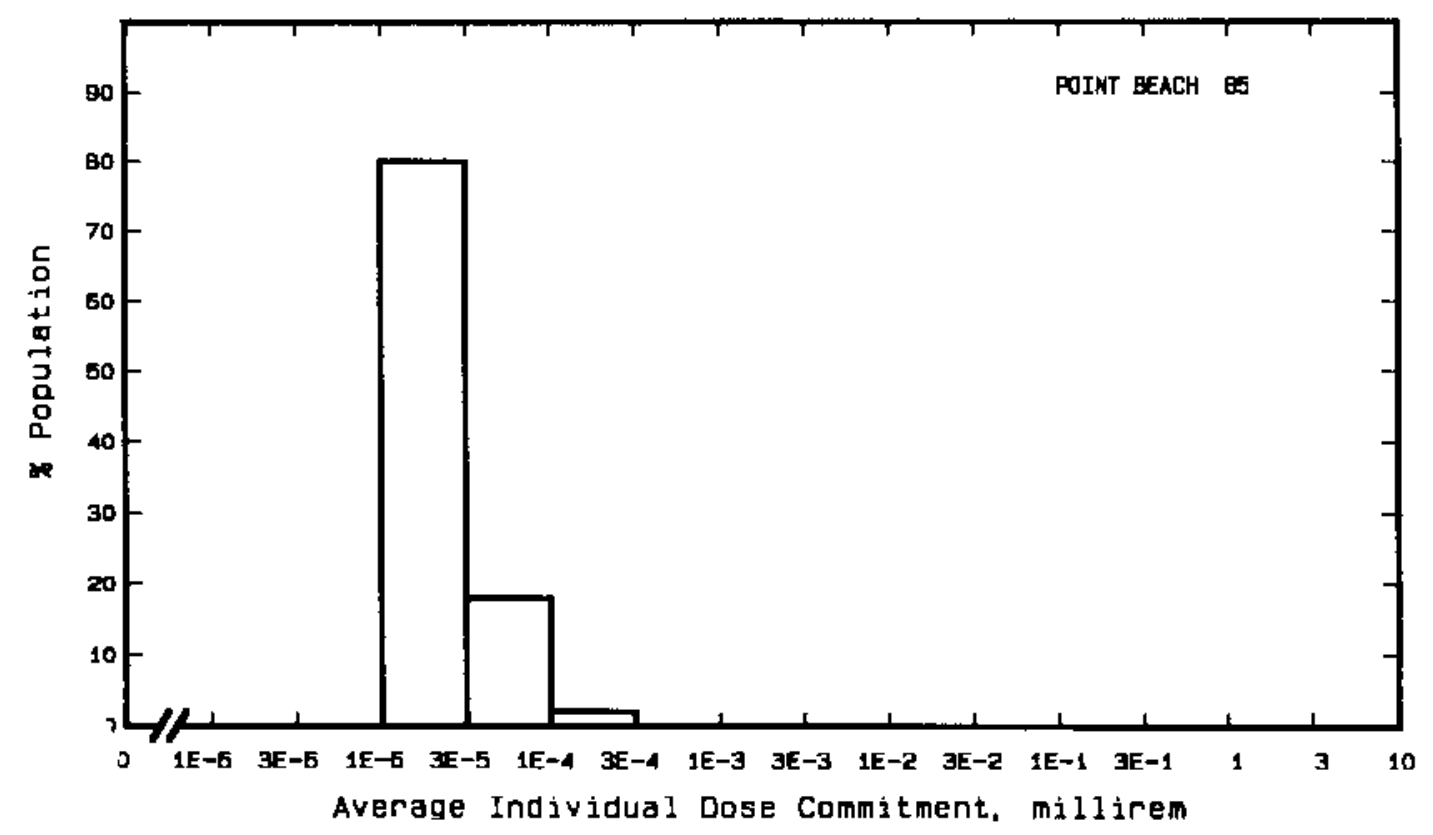


Site: PRAIRIE ISLAND

RED WING, MINNESOTA

Location: $\quad \mathrm{N} 44.6219^{\circ} \quad$ W $92.6331^{\circ}$

POPULATION DATA

Total Population Within 2-to-80-km Region: 2.2E6

Major Metropolitan Centers Within Region:

Center Population Location

Minneapolis-St. Paul SMSA 2,100,000 $63 \mathrm{~km} \quad$ NW

Rochester SMSA $\quad 95,000 \quad 68 \mathrm{~km} \quad$ SSE

Owatonna $\quad 19,000 \quad 77 \mathrm{~km} \quad \mathrm{SW}$

Faribault $\quad 16,000 \quad 63 \mathrm{~km} \quad \mathrm{SW}$

Redwing $14,000 \quad 10 \mathrm{~km} \quad \mathrm{SE}$

SITE-SPECIFIC DATA - AIRBORNE PATHWAYS

Average Annual State Production Veg: 1.2E8 kilogram

of Crops and Animal Products

Milk: 4.0E8 liter

In $80-\mathrm{km}$ Radius Circle

Meat: $1.0 \mathrm{E} 8 \mathrm{ki}$ logram

Regional Productivity Factor: 1

Animal Grazing Factor: $\quad 0.5$

Meteorology Period of Record: 22 MAR 74 - 21 MAR 75 Recovery: 65\%

SITE-SPECIFIC DATA - WATERBDRNE PATHWAYS via MISSISSIPPI RIVER

Average River Flow

at Site: $15,000 \mathrm{ft}^{3} / \mathrm{s}$

Drinking Water:

Exposed Population: None

Fish:

Edible Harvest: $6.8 \mathrm{E} 5 \mathrm{~kg} / \mathrm{yr}$

Dilution Factor: 1 


\section{POPULATION DOSE-COMMITMENT ESTIMATES AND \\ AVERAGE INDIVIDUAL DOSE-COMMITMENT HISTOGRAM FOR}

PRAIRIE ISLAND 1 AND 2

Dose Commitments (person-rem) from Waterborne Pathways

Total Body

$\underline{G I-L I I}$

Thyroid

Bone

Liver

Infant

$0.0 \mathrm{E}+00$

$0.0 E+00$

$0.0 \mathrm{E}+00$

$0.0 \mathrm{E}+00$

$0.0 \mathrm{E}+00$

Child

5. $0 \mathrm{E}-04$

4. $4 \mathrm{E}-04$

4.7E-04

6. $0 \mathrm{E}-04$

$1.0 \mathrm{E}-03$

Teen

5. 5E-04

4.5E-04

4. $1 \mathrm{E}-04$

3. $6 \mathrm{E}-04$

8. $6 \mathrm{E}-04$

Adult

4. $9 \mathrm{E}-03$

3. $7 \mathrm{E}-03$

3. $2 \mathrm{E}-03$

2. $0 \mathrm{E}-03$

5. $8 \mathrm{E}-03$

TOTAL

5. $9 \mathrm{E}-03$

4. 5E-03

4. $0 \mathrm{E}-03$

3. $0 \mathrm{E}-03$

7.7E-03

Dose Commitments (person-rem) from Airborne Pathways

Iotal Body GI-LII Thyroid Bone Liver Lung

$\begin{array}{lllllll}\text { Infant } & 4.8 \mathrm{E}-04 & 4.7 \mathrm{E}-04 & 1.1 \mathrm{E}-02 & 4.8 \mathrm{E}-05 & 5.0 \mathrm{E}-04 & 4.7 \mathrm{E}-04 \\ \text { Child } & 7.4 \mathrm{E}-03 & 7.3 \mathrm{E}-03 & 7.7 \mathrm{E}-02 & 4.4 \mathrm{E}-04 & 7.5 \mathrm{E}-03 & 7.3 \mathrm{E}-03 \\ \text { Teen } & 4.7 \mathrm{E}-03 & 4.6 \mathrm{E}-03 & 3.1 \mathrm{E}-02 & 2.3 \mathrm{E}-04 & 4.7 \mathrm{E}-03 & 4.7 \mathrm{E}-03 \\ \text { Adult } & 2.5 \mathrm{E}-02 & 2.5 \mathrm{E}-02 & 1.1 \mathrm{E}-01 & 1.2 \mathrm{E}-03 & 2.5 \mathrm{E}-02 & 2.5 \mathrm{E}-02 \\ \text { TOTAL } & 3.8 \mathrm{E}-02 & 3.8 \mathrm{E}-02 & 2.3 \mathrm{E}-01 & 1.9 \mathrm{E}-03 & 3.8 \mathrm{E}-02 & 3.8 \mathrm{E}-02\end{array}$

Production/Consumption factors:

Produce: 0.28

Milk: 1.4

Meat: $\quad 0.59$

Fraction of Population Receiving an Average Individual

Total-Body Dose Commitment from Airborne Pathways

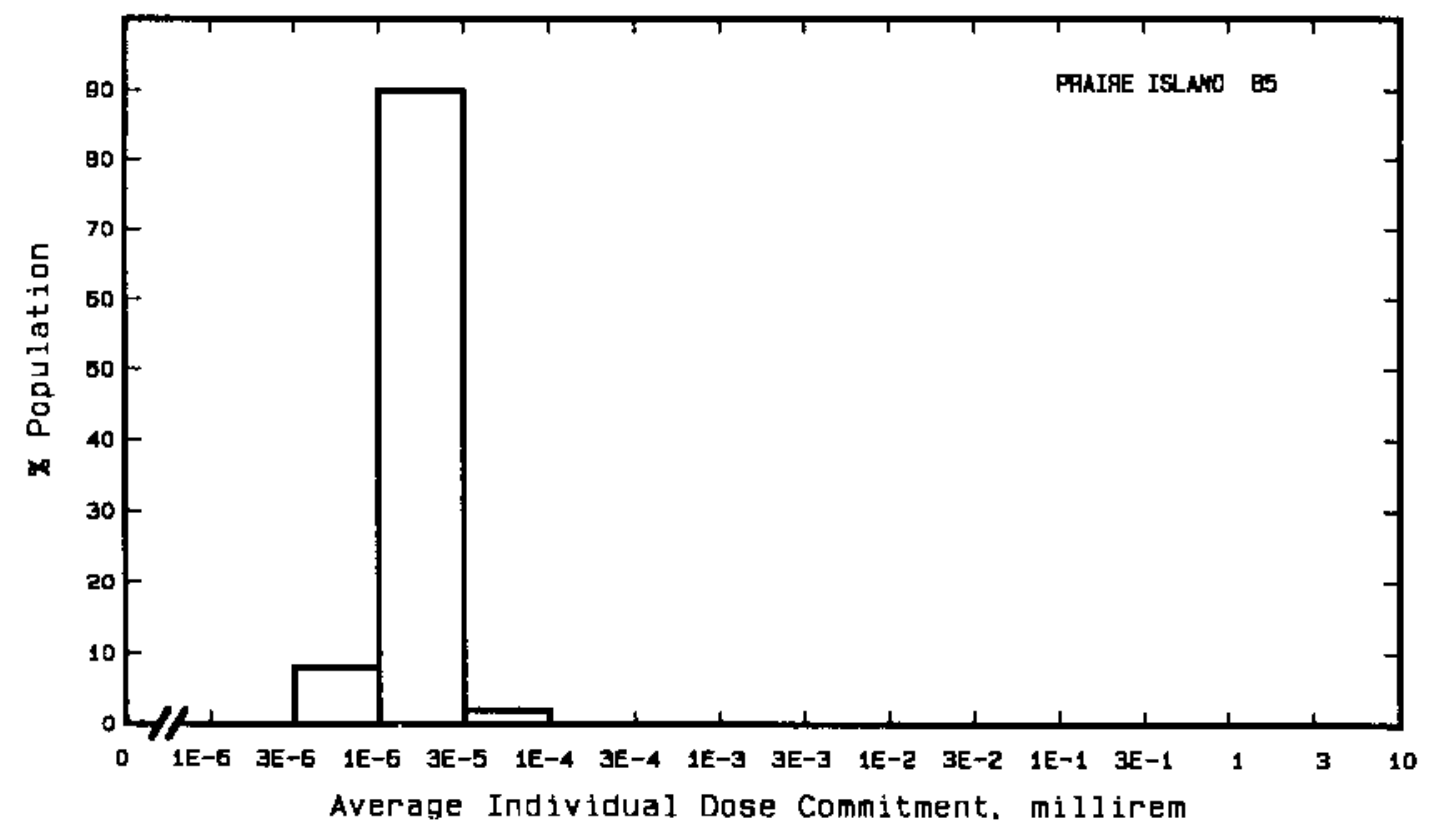


Site: QUAD CITIES

ROCK ISLAND, ILLINOIS

Location: $\quad$ N $41.7261^{\circ} \quad$ W $90.3100^{\circ}$

POPULATION DATA

Total Population Within 2-to-80-km Region: 7.2E5

Major Metropolitan Centers Within Region:

Center Population Location

Davenport-Rock Island-Moline SMSA $380,000 \quad 30 \mathrm{~km} \quad$ SW

Muscatine

23,000

Sterling

16,000

Dixon

16,000

Kewanee

15,000

$70 \mathrm{~km} \quad \mathrm{WSW}$

$52 \mathrm{~km} \quad \mathrm{E}$

$70 \mathrm{~km} \quad \mathrm{E}$

$62 \mathrm{~km} \quad$ SSE

SITE-SPECIFIC DATA - AIRBORNE PATHWAYS

Average Annual State Production

of Crops and Animal Products

In 80-km Radius Circle

Regional Productivity Factor:

Animal Grazing Factor:
Veg: $1.1 \mathrm{E} 8 \mathrm{kilogram}$

MiTk: 1.8E8 liter

Meat: $1.9 \mathrm{E} 8$ kilogram

1

0.5

Meteorology Period of Record: I JAN 74 - 31 DEC 75 Recovery: $88 \%$

\section{SITE-SPECIFIC DATA - WATERBORNE PATHWAYS via MISSISSIPPI RIVER}

Drinking Water:

Fish:
Average River Flow at Site: $47,000 \mathrm{ft}^{3} / \mathrm{s}$

Exposed Population: 380,000 (a)

Dilution Factor: 1

Edible Harvest: $2.1 \mathrm{E}{ }^{(b)} \mathrm{kg} / \mathrm{yr}$

Dilution Factor: 0.5 (b)

(a) All people in Davenport SMSA assumed to drink river water.

(b) Assumes $1 / 2$ fish harvest caught below plant. 
POPULATION DOSE-COMMITMENT ESTIMATES AND

AVERAGE INDIVIDUAL DOSE-COMMITMENT HISTOGRAM FOR

QUAD CITIES 1 AND 2

Dose Commitments (person-rem) from Waterborne Pathways

Total Body GI-LII $\underline{\text { Thyroid }}$ Bone Liver

$\begin{array}{llllll}\text { Infant } & 8.2 \mathrm{E}-03 & 5.0 \mathrm{E}-04 & 3.9 \mathrm{E}-04 & 4.4 \mathrm{E}-02 & 1.8 \mathrm{E}-02 \\ \text { Child } & 3.4 \mathrm{E}-01 & 1.7 \mathrm{E}-02 & 2.9 \mathrm{E}-03 & 1.8 \mathrm{E}+00 & 1.4 \mathrm{E}+00 \\ \text { Teen } & 4.4 \mathrm{E}-01 & 2.2 \mathrm{E}-02 & 9.4 \mathrm{E}-04 & 9.5 \mathrm{E}-01 & 1.1 \mathrm{E}+00 \\ \text { Adult } & 4.7 \mathrm{E}+00 & 1.8 \mathrm{E}-01 & 6.7 \mathrm{E}-03 & 5.9 \mathrm{E}+00 & 6.4 \mathrm{E}+00 \\ \text { TOTAL } & 5.5 \mathrm{E}+00 & 2.2 \mathrm{E}-01 & 1.1 \mathrm{E}-02 & 8.7 \mathrm{E}+00 & 8.9 \mathrm{E}+00\end{array}$

Dose Commitments (person-rem) from Airborne Pathways

Total Body GI-LII Thyroid Bone Liver Lung

$\begin{array}{lllllll}\text { Infant } & 2.7 \mathrm{E}-03 & 2.6 \mathrm{E}-03 & 2.0 \mathrm{E}-02 & 2.9 \mathrm{E}-03 & 2.9 \mathrm{E}-03 & 2.8 \mathrm{E}-03 \\ \text { Child } & 3.2 \mathrm{E}-02 & 3.1 \mathrm{E}-02 & 2.0 \mathrm{E}-01 & 4.2 \mathrm{E}-02 & 3.4 \mathrm{E}-02 & 3.4 \mathrm{E}-02 \\ \text { Teen } & 2.3 \mathrm{E}-02 & 2.3 \mathrm{E}-02 & 9.0 \mathrm{E}-02 & 2.5 \mathrm{E}-02 & 2.3 \mathrm{E}-02 & 2.5 \mathrm{E}-02 \\ \text { Adult } & 1.4 \mathrm{E}-01 & 1.4 \mathrm{E}-01 & 3.9 \mathrm{E}-01 & 1.4 \mathrm{E}-01 & 1.4 \mathrm{E}-01 & 1.4 \mathrm{E}-01 \\ \text { TOTAL } & 1.9 \mathrm{E}-01 & 1.9 \mathrm{E}-01 & 6.9 \mathrm{E}-01 & 2.1 \mathrm{E}-01 & 2.0 \mathrm{E}-01 & 2.0 \mathrm{E}-01\end{array}$

Production/Consumption factors:

Produce: 0.81

Milk: 1.9

Meat: 3.2

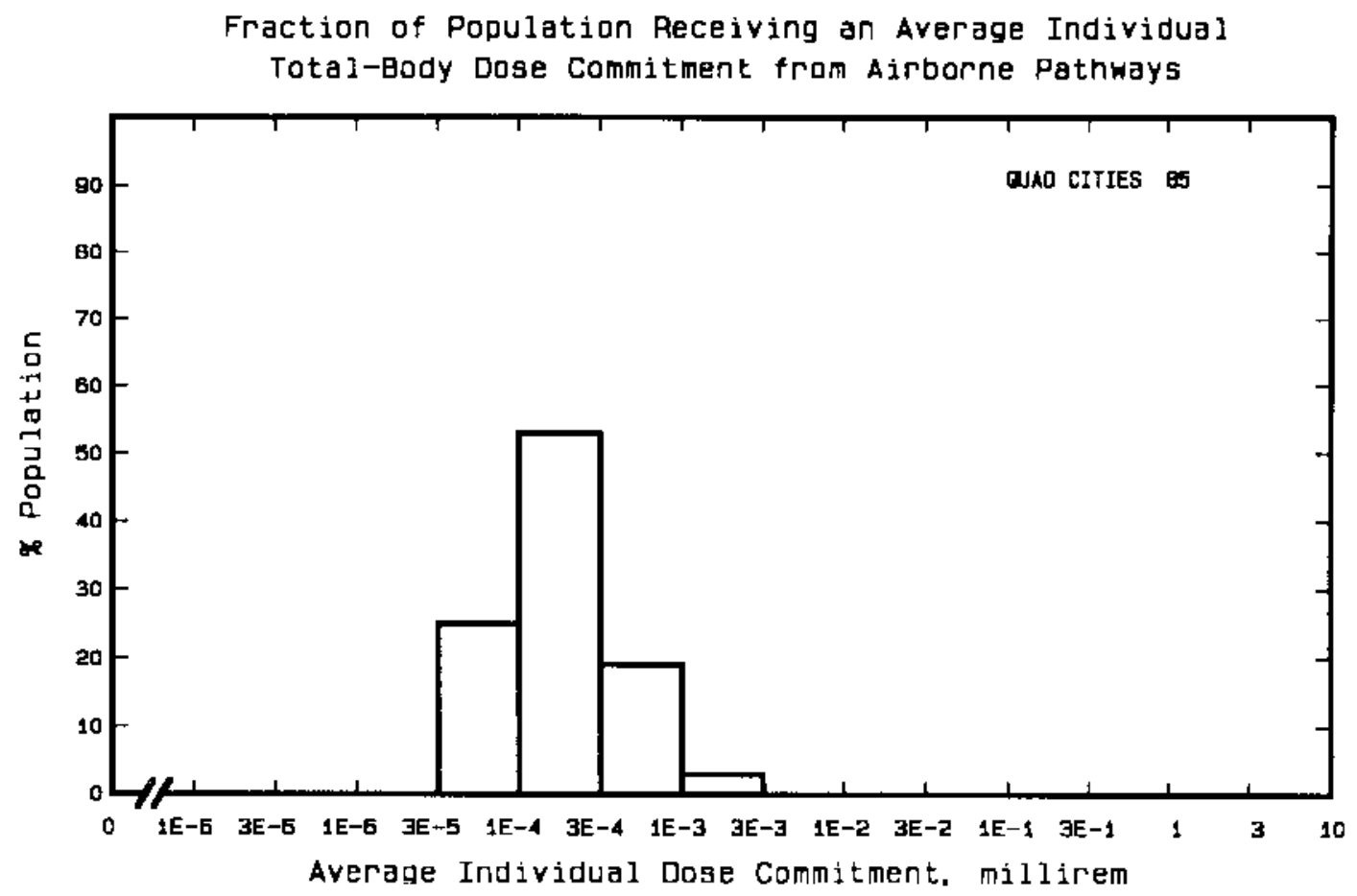


Site: RANCHO SECO

SACRAMENTO COUNTY, CALIFORNIA

Location: $\quad \mathrm{N} 38.3444^{\circ} \quad$ W $121.1200^{\circ}$

POPULATION DATA

Total Population Within 2-to-80-km Region: 2.0E6

Major Metropolitan Centers Within Region:

\begin{tabular}{lrrl}
\multicolumn{1}{c}{ Center } & Population & \multicolumn{2}{c}{ Location } \\
Sacramento SMSA & $1,300,000$ & & \\
Stockton SMSA & 420,000 & $42 \mathrm{~km}$ & NW \\
Modesto SMSA & 310,000 & $45 \mathrm{~km}$ & SSW \\
Antioch & 48,000 & $79 \mathrm{~km}$ & S \\
Davis & 41,000 & $71 \mathrm{~km}$ & WSW \\
& & $58 \mathrm{~km}$ & WNW
\end{tabular}

SITE-SPECIFIC DATA - AIRBORNE PATHWAYS

Average Annual State Production

of Crops and Animal Products

In $\mathbf{B 0}-\mathrm{km}$ Radius Circle

Regional Productivity Factor:

Animal Grazing Factor:

Meteorology Period of Record: 1 FEB 75 - 31 JAN 76 Recovery: $98 \%$
Veg: $4.8 E 7 \mathrm{ki}$ logram

Mi]k: 2.3EB liter

Meat: 5.0E7 kilogram

1

0.9

SITE SPECIFIC DATA - WATERBORNE PATHWAYS via COSUMNES \& MOKELUMNE

RIVERS

Average Dilution Flow

from Plant: $11 \mathrm{ft}^{3} / \mathrm{s}$

Drinking Water:

Exposed Population: None

Fish:

Edible Harvest: (a)

Dilution Factor: 0.005

(a) One percent of population is assumed to obta in fish from river. Average individual consumption rate of $7.3 \mathrm{~kg} / \mathrm{yr}$ given in $\mathrm{FES}$ (1973) used in lieu of catch data. 


\section{POPULATION OOSE-COMMITMENT ESTIMATES AND}

AVERAGE INDIVIOUAL DOSE-COMMITMENT HISTOGRAM FOR

\section{RANCHO SECO}

Dose Commitments (person-rem) from Waterborne Pathways

Total Body GI-LII Thyroid Bone Liver

$\begin{array}{llllll}\text { Infant } & 0.0 \mathrm{E}+00 & 0.0 \mathrm{E}+00 & 0.0 \mathrm{E}+00 & 0.0 \mathrm{E}+00 & 0.0 \mathrm{E}+00 \\ \text { Child } & 3.0 \mathrm{E}-03 & 1.7 \mathrm{E}-04 & 8.7 \mathrm{E}-05 & 1.5 \mathrm{E}-02 & 1.7 \mathrm{E}-02 \\ \text { Teen } & 5.8 \mathrm{E}-03 & 2.6 \mathrm{E}-04 & 7.5 \mathrm{E}-05 & 9.1 \mathrm{E}-03 & 1.5 \mathrm{E}-02 \\ \text { Adult } & 6.3 \mathrm{E}-02 & 2.1 \mathrm{E}-03 & 5.8 \mathrm{E}-04 & 5.2 \mathrm{E}-02 & 8.7 \mathrm{E}-02 \\ \text { TOTAL } & 7.2 \mathrm{E}-02 & 2.5 \mathrm{E}-03 & 7.4 \mathrm{E}-04 & 7.7 \mathrm{E}-02 & 1.2 \mathrm{E}-01\end{array}$

Dose Commitments (person-rem) from Airborne Pathways

Total Body GI-LII Thyroid Bone Liver

$\begin{array}{lllllll}\text { Infant } & 2.7 \mathrm{E}-03 & 2.6 \mathrm{E}-03 & 1.1 \mathrm{E}-02 & 2.5 \mathrm{E}-03 & 2.8 \mathrm{E}-03 & 2.8 \mathrm{E}-03 \\ \text { Child } & 3.0 \mathrm{E}-02 & 3.0 \mathrm{E}-02 & 7.5 \mathrm{E}-01 & 2.8 \mathrm{E}-02 & 3.1 \mathrm{E}-02 & 3.3 \mathrm{E}-02 \\ \text { Teen } & 2.2 \mathrm{E}-02 & 2.2 \mathrm{E}-02 & 3.9 \mathrm{E}-02 & 2.0 \mathrm{E}-02 & 2.2 \mathrm{E}-02 & 2.5 \mathrm{E}-02 \\ \text { Adult } & 1.3 \mathrm{E}-01 & 1.3 \mathrm{E}-01 & 1.9 \mathrm{E}-01 & 1.2 \mathrm{E}-01 & 1.3 \mathrm{E}-01 & 1.4 \mathrm{E}-01 \\ \text { TOTAL } & 1.8 \mathrm{E}-01 & 1.8 \mathrm{E}-01 & 3.1 \mathrm{E}-01 & 1.7 \mathrm{E}-01 & 1.9 \mathrm{E}-01 & 2.0 \mathrm{E}-01\end{array}$

Production/Consumption factors:

Produce: 0.12 Milk: $0.90 \quad$ Meat: 0.32

Fraction of Population Receiving an Average Individual Total-Body Dose Commitment from Airoorne Pathways

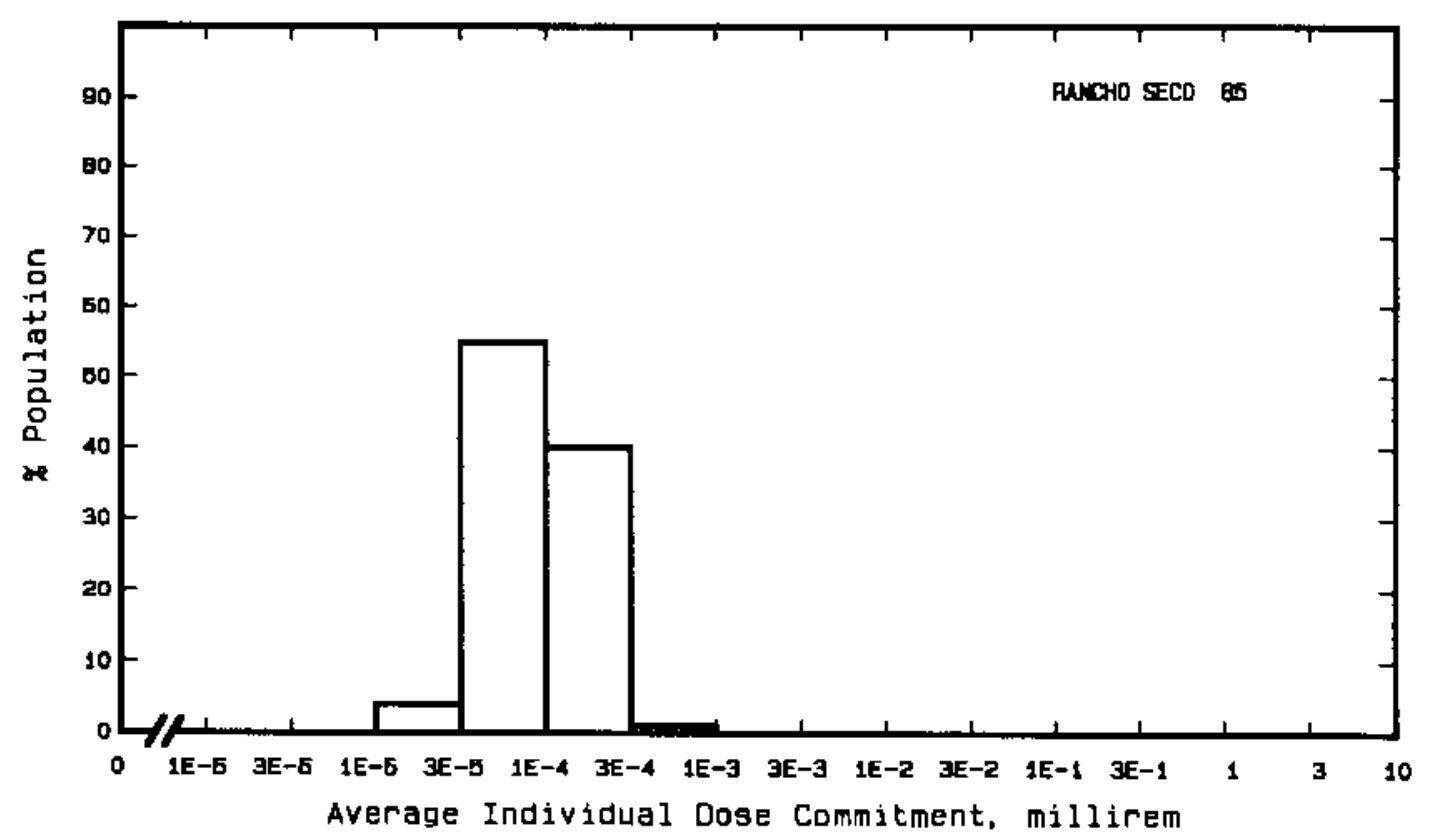


Site: H. B. ROBINSON

Location: N $34.4858^{\circ}$
HARTSVILLE, SOUTH CAROLINA

W $80.1586^{\circ}$

POPULATION DATA

Total Population Within 2-to-80-km Region: 7.1E5

Major Metropolitan Centers Within Region:

\begin{tabular}{lcccc} 
Center & Population & & \multicolumn{2}{c}{ Location } \\
\cline { 2 - 6 } & & & \\
Florence & 32,000 & $42 \mathrm{~km}$ & ESE \\
Sunter & 27,000 & $56 \mathrm{~km}$ & SSW \\
Monroe & 14,000 & $74 \mathrm{~km}$ & NNW \\
Lancaster & 10,000 & $66 \mathrm{~km}$ & WNW
\end{tabular}

\section{SITE-SPECIFIC DATA - AIRBORNE PATHWAYS}

Average Annual State Production

of Crops and Animal Products

In $80-\mathrm{km}$ Radius Circle

Regional Productivity Factor:

Animal Grazing Factor:
Veg: 7.4E6 kilogram

Milk: 5.7E7 liter

Meat: $5.0 \mathrm{E} 7$ kilogram

1

0.8

Meteorology Period of Record: 1 JAN 75 - 31 DEC 75 Recovery: $94 \%$

SITE-SPECIFIC DATA - WATERBORNE PATHWAYS via LAKE ROBINSON

Average Dilution Flow

from Plant: $1,100 \mathrm{ft}^{3} / \mathrm{s}$

Drinking Water:

Exposed Population: None

Fish:

Edible Harvest: $1.8^{(a)} \mathrm{kg} / \mathrm{yr}$

Difution Factor: $0.02^{(b)}$

(a) Average individual consumption rate as given in the FES (1975) used in lieu of catch data.

(b) Ten percent of population consumes fish taken from water diluted by a factor of 0.2 according to FES. 
POPULATION DOSE-COMMITMENT ESTIMATES AND
AVERAGE INDIVIDUAL DOSE-COMMITMENT HISTOGRAM FOR

H. B. ROBINSON

Dose Commitments (person-rem) from Waterborne Pathways

$\underline{\text { Total Body GI-LII } \underline{\text { Thyroid }} \text { Bone Liver }}$

$\begin{array}{llllll}\text { Infant } & 0.0 \mathrm{E}+00 & 0.0 \mathrm{E}+00 & 0.0 \mathrm{E}+00 & 0.0 \mathrm{E}+00 & 0.0 \mathrm{E}+00 \\ \text { Child } & 6.7 \mathrm{E}-03 & 3.9 \mathrm{E}-04 & 3.2 \mathrm{E}-04 & 3.3 \mathrm{E}-02 & 3.8 \mathrm{E}-02 \\ \text { Teen } & 1.3 \mathrm{E}-02 & 6.9 \mathrm{E}-04 & 2.5 \mathrm{E}-04 & 2.0 \mathrm{E}-02 & 3.2 \mathrm{E}-02 \\ \text { Adult } & 1.4 \mathrm{E}-01 & 5.8 \mathrm{E}-03 & 1.7 \mathrm{E}-03 & 1.1 \mathrm{E}-01 & 1.9 \mathrm{E}-01 \\ \text { TOTAL } & 1.6 \mathrm{E}-01 & 6.9 \mathrm{E}-03 & 2.3 \mathrm{E}-03 & 1.7 \mathrm{E}-01 & 2.6 \mathrm{E}-01\end{array}$

Dose Commitments (person-rem) from Airborne Pathways

Toth1 Body GI-LII Thyroid Bone Liver lung

$\begin{array}{lllllll}\text { Infant } & 7.1 \mathrm{E}-04 & 7.1 \mathrm{E}-04 & 6.0 \mathrm{E}-03 & 4.8 \mathrm{E}-04 & 7.3 \mathrm{E}-04 & 7.5 \mathrm{E}-04 \\ \text { ChiTd } & 8.9 \mathrm{E}-03 & 8.9 \mathrm{E}-03 & 3.9 \mathrm{E}-02 & 5.2 \mathrm{E}-03 & 9.0 \mathrm{E}-03 & 9.5 \mathrm{E}-03 \\ \text { Teen } & 6.3 \mathrm{E}-03 & 6.3 \mathrm{E}-03 & 1.8 \mathrm{E}-02 & 3.7 \mathrm{E}-03 & 6.4 \mathrm{E}-03 & 7.1 \mathrm{E}-03 \\ \text { Adu1t } & 3.8 \mathrm{E}-02 & 3.8 \mathrm{E}-02 & 8.0 \mathrm{E}-02 & 2.3 \mathrm{E}-02 & 3.8 \mathrm{E}-02 & 4.0 \mathrm{E}-02 \\ \text { TOTAL } & 5.4 \mathrm{E}-02 & 5.3 \mathrm{E}-02 & 1.4 \mathrm{E}-01 & 3.2 \mathrm{E}-02 & 5.4 \mathrm{E}-02 & 5.7 \mathrm{E}-02\end{array}$

Production/Consumption factors:

Produce: $0.054 \quad$ Milk: $0.62 \quad$ Meat: 0.88

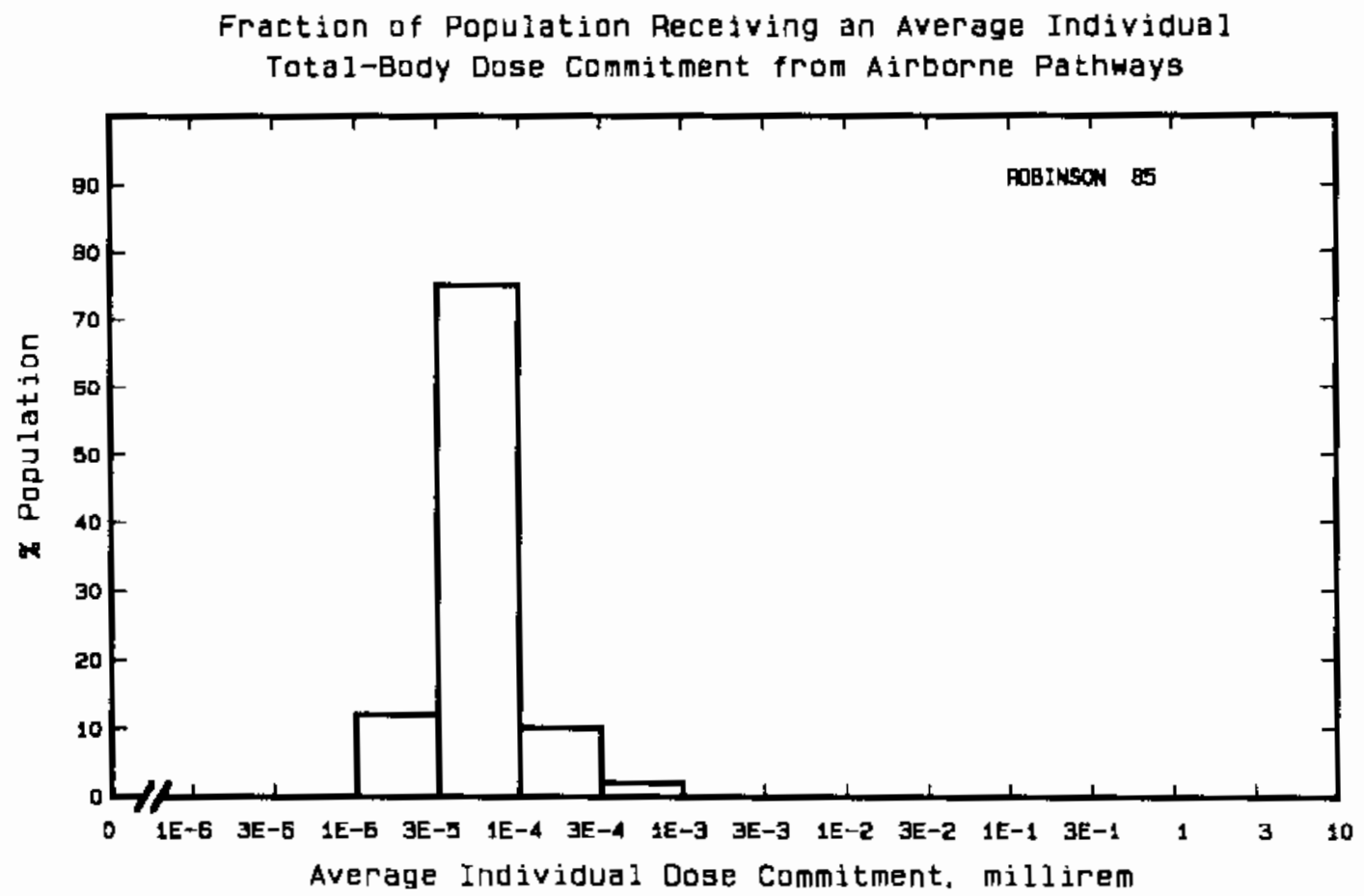


Site: ST. LUCIE

FORT PIERCE, FLORIDA

Location: $\quad$ N $27.3486^{\circ} \quad$ W $80.2464^{\circ}$

POPULATION DATA

Total Population Within 2-to-80-km Region: 6.7E5

Major Metropolitan Centers Within Region:

Center

West Palm Beach

Ft. Pierce

Riviera Beach

Vero Beach

Palm Beach
Population

73,000

39,000

30,000

19,000

12,000
Location

$73 \mathrm{~km} \quad$ SSE

$14 \mathrm{~km} \quad \mathrm{NW}$

$65 \mathrm{~km} \quad$ SSE

$36 \mathrm{~km} \quad \mathrm{NNW}$

$72 \mathrm{~km} \quad$ SSE

SITE-SPECIFIC DATA - AIRBORNE PATHWAYS

Average Annual State Production

of Crops and Animal Products

In 80-km Radius Circle

Regional Productivity Factor:

Animal Grazing Factor:
Veg: $2.8 \mathrm{E} 7$ kilogram

Milk: 1.1E8 liter

Meat: $7.2 E 7$ kilogram

0.5

1

Meteorology Period of Record: 1 JAN 76 - 31 DEC 76 Recovery: 92\%

SITE-SPECIFIC DATA - WATERBORNE PATHWAYS via ATLANTIC OCEAN

Fish:

Average Dilution Flow

from Plant: $1,100 \mathrm{ft}^{3} / \mathrm{s}$

Edible Harvest: $2.6 \mathrm{E} 5 \mathrm{~kg} / \mathrm{yr}$

Invertebrates:

Dilution Factor: $0.005^{(a)}$

Edible Harvest: $2.7 \mathrm{E} 4 \mathrm{~kg} / \mathrm{yr}$

Dilution Factor: $0.005(\mathrm{a})$

(a) Dilution factors as given in FES (1973). 


\section{POPULATION DOSE-COMMITMENT ESTIMATES AND \\ AVERAGE INDIVIDUAL DOSE-COMMITMENT HISTOGRAM FOR \\ SAINT LUCIE 1 AND 2}

Dose Commitments (person-rem) from Waterborne Pathways

Iotal Body GI-LII $\underline{\text { Thyroid }}$ Bone

$\begin{array}{llllll}\text { Infant } & 0.0 \mathrm{E}+00 & 0.0 \mathrm{E}+00 & 0.0 \mathrm{E}+00 & 0.0 \mathrm{E}+00 & 0.0 \mathrm{E}+00 \\ \text { Child } & 1.5 \mathrm{E}-03 & 6.4 \mathrm{E}-03 & 9.8 \mathrm{E}-04 & 6.2 \mathrm{E}-03 & 4.3 \mathrm{E}-03 \\ \text { Teen } & 1.2 \mathrm{E}-03 & 1.3 \mathrm{E}-02 & 7.0 \mathrm{E}-04 & 3.5 \mathrm{E}-03 & 3.4 \mathrm{E}-03 \\ \text { Adult } & 9.3 \mathrm{E}-03 & 1.1 \mathrm{E}-01 & 4.6 \mathrm{E}-03 & 2.0 \mathrm{E}-02 & 2.0 \mathrm{E}-02 \\ \text { TOTAL } & 1.2 \mathrm{E}-02 & 1.3 \mathrm{E}-01 & 6.3 \mathrm{E}-03 & 3.0 \mathrm{E}-02 & 2.8 \mathrm{E}-02\end{array}$

Dose Commitments (person-rem) from Airborne Pathways

Iotal Body GI-LII Thyroid Bone Liver Lung

$\begin{array}{lllllll}\text { Infant } & 2.3 \mathrm{E}-02 & 2.3 \mathrm{E}-02 & 1.4 \mathrm{E}-01 & 2.2 \mathrm{E}-02 & 2.3 \mathrm{E}-02 & 2.4 \mathrm{E}-02 \\ \text { Chi1d } & 2.6 \mathrm{E}-01 & 2.6 \mathrm{E}-01 & 9.7 \mathrm{E}-01 & 2.4 \mathrm{E}-01 & 2.7 \mathrm{E}-01 & 2.8 \mathrm{E}-01 \\ \text { Teen } & 1.9 \mathrm{E}-01 & 1.9 \mathrm{E}-01 & 4.9 \mathrm{E}-01 & 1.8 \mathrm{E}-01 & 1.9 \mathrm{E}-01 & 2.2 \mathrm{E}-01 \\ \text { Adult } & 1.2 \mathrm{E}+00 & 1.2 \mathrm{E}+00 & 2.2 \mathrm{E}+00 & 1.1 \mathrm{E}+00 & 1.2 \mathrm{E}+00 & 1.2 \mathrm{E}+00 \\ \text { TOTAL } & 1.6 \mathrm{E}+00 & 1.6 \mathrm{E}+00 & 3.8 \mathrm{E}+00 & 1.5 \mathrm{E}+00 & 1.6 \mathrm{E}+00 & 1.8 \mathrm{E}+00\end{array}$

Production/Consumption factors:

Produce: 0.11 Milk: 0.64 Meat: 0.68

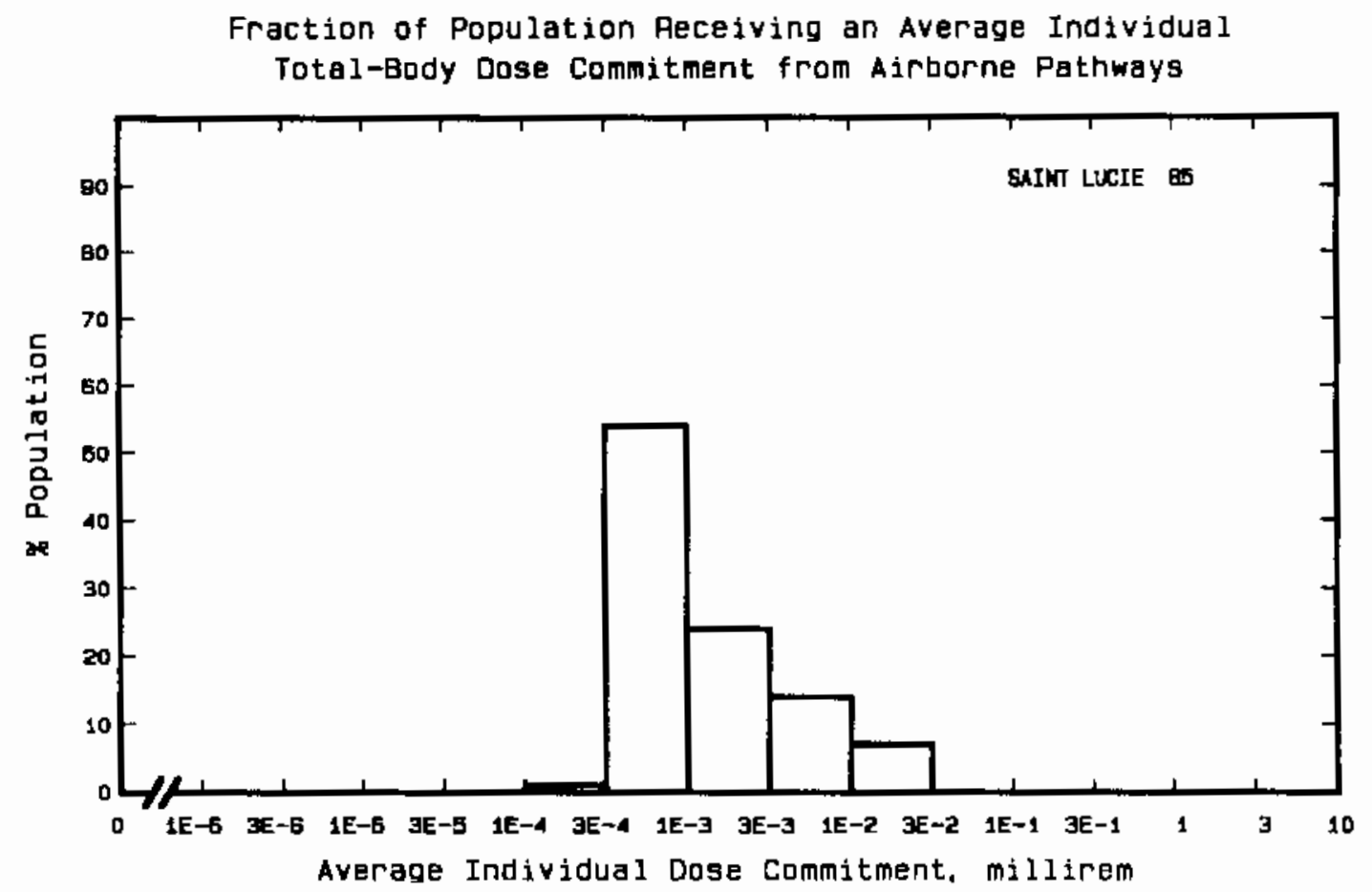


Site: SALEM

SALEM, NEW JERSEY

Location: $\quad$ N $39.4628^{\circ}$ W $75.5358^{\circ}$

POPULATION DATA

Total Population Within 2-to-80-km Region: 4.8E6

Major Metropolitan Centers Within Region:

Center

Philadelphia SMSA

Wilmington SMSA

Vineland-Millville-Bridgeton SMSA

Chester

Norristown
Population

$4,800,100$

410,000

130,000

47,000

35,000

\section{Location}

$63 \mathrm{~km}$ NNE.

$32 \mathrm{~km} \quad \mathrm{NNW}$.

$48 \mathrm{~km} \quad \mathrm{E}$

$45 \mathrm{~km} \quad \mathrm{NNE}$

$74 \mathrm{~km} \quad \mathrm{~N}$

SITE-SPECIFIC DATA - AIRBORNE PATHWAYS

Average Annual State Production

of Crops and Animal Products

In 80-km Radius Circle

Regional Productivity Factor:

Animal Grazing Factor:
Veg: $7.4 \mathrm{E} 7 \mathrm{kilogram}$

Milk: 2.7E8 liter

Meat: $2.4 \mathrm{E} 7 \mathrm{kilogram}$

0.9

0.6

Meteorology Period of Record: 1 JUN 70 - 31 MAY 71 Recovery: 95\%

Average River Flow at Site: $16,000 \mathrm{ft}^{3} / \mathrm{s}$

Fish:

Invertebrates: (a)
Edible Harvest: $3.6 \mathrm{E} 5 \mathrm{~kg} / \mathrm{yr}$

Dilution Factor: 1

Edible Harvest: $1.6 \mathrm{E} 5 \mathrm{~kg} / \mathrm{yr}$ Dilution Factor: 1

(a) Environment primarily salt water so invertebrates considered in lieu of drinking water. 


\section{POPULATION DOSE-COMMITMENT ESTIMATES AND}

AVERAGE INDIVIDUAL DOSE-COMMITMENT HISTOGRAM FOR

\section{SALEM 1 AND 2}

Dose Commitments (person-rem) from Waterborne Pathways

Total Body GI-LII Thyroid Bone Liver

$\begin{array}{llllll}\text { Infant } & 0.0 \mathrm{E}+00 & 0.0 \mathrm{E}+00 & 0.0 \mathrm{E}+00 & 0.0 \mathrm{E}+00 & 0.0 \mathrm{E}+00 \\ \text { Child } & 8.0 \mathrm{E}-02 & 9.5 \mathrm{E}-02 & 2.6 \mathrm{E}-02 & 3.2 \mathrm{E}-01 & 2.0 \mathrm{E}-01 \\ \text { Teen } & 5.3 \mathrm{E}-02 & 1.9 \mathrm{E}-01 & 1.8 \mathrm{E}-02 & 1.8 \mathrm{E}-01 & 1.5 \mathrm{E}-01 \\ \text { Adult } & 3.5 \mathrm{E}-01 & 1.6 \mathrm{E}+00 & 1.2 \mathrm{E}-01 & 1.0 \mathrm{E}+00 & 8.6 \mathrm{E}-01 \\ \text { TOTAL } & 4.8 \mathrm{E}-01 & 1.9 \mathrm{E}+00 & 1.7 \mathrm{E}-01 & 1.5 \mathrm{E}+00 & 1.2 \mathrm{E}+00\end{array}$

Dose Commitments (person-rem) from Airborne Pathways

Total Body GI-LII Thyroid Bone Liver Lung

$\begin{array}{lllllll}\text { Infant } & 1.1 \mathrm{E}-03 & 1.1 \mathrm{E}-03 & 8.4 \mathrm{E}-03 & 7.4 \mathrm{E}-04 & 1.2 \mathrm{E}-03 & 1.3 \mathrm{E}-03 \\ \text { Child } & 1.5 \mathrm{E}-02 & 1.5 \mathrm{E}-02 & 5.7 \mathrm{E}-02 & 8.1 \mathrm{E}-03 & 1.5 \mathrm{E}-02 & 1.7 \mathrm{E}-02 \\ \text { Teen } & 1.0 \mathrm{E}-02 & 1.0 \mathrm{E}-02 & 2.8 \mathrm{E}-02 & 5.6 \mathrm{E}-03 & 1.0 \mathrm{E}-02 & 1.3 \mathrm{E}-02 \\ \text { Adult } & 5.9 \mathrm{E}-02 & 6.1 \mathrm{E}-02 & 1.2 \mathrm{E}-01 & 3.3 \mathrm{E}-02 & 6.0 \mathrm{E}-02 & 7.0 \mathrm{E}-02 \\ \text { TOTAL } & 8.5 \mathrm{E}-02 & 8.7 \mathrm{E}-02 & 2.1 \mathrm{E}-01 & 4.7 \mathrm{E}-02 & 8.7 \mathrm{E}-02 & 1.0 \mathrm{E}-01\end{array}$

Production/Consumption factors:

Produce: 0.071 Milk: 0.39 Meat: 0.056

Fraction of Population Receiving an Average Individual

Tota3-Body Dose commitment from Airborne Patnways

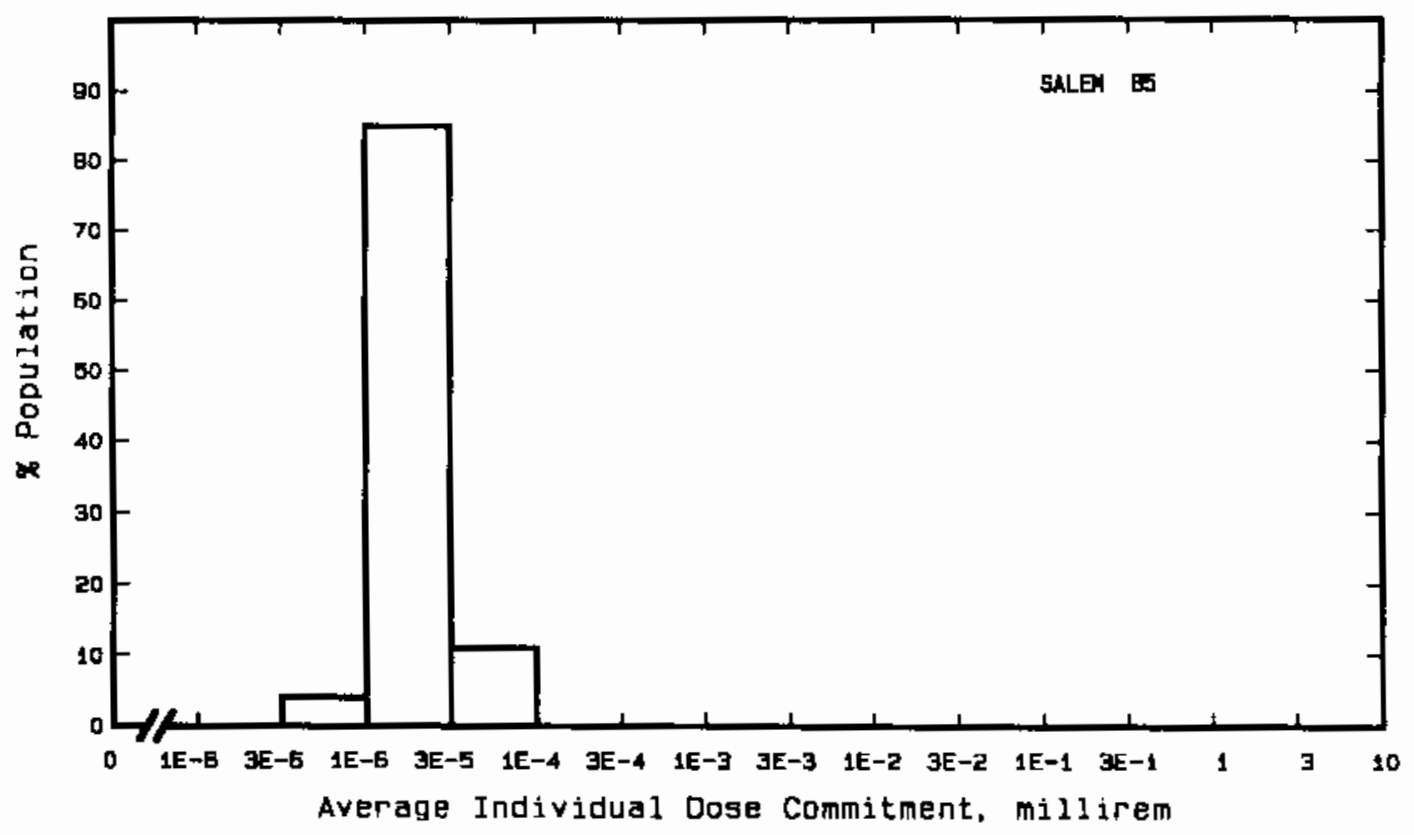


Site: SAN ONOFRE

CAMP PENDLETON, CALIFORNIA

Location: $\quad$ N $33.3703^{\circ} \quad$ W $117.5569^{\circ}$

POPULATION DATA

Total Population Within 2-to-80-km Region: 5.2E6

Major Metropolitan Centers Within Region:

Center

Population

Location

San Diego SMSA

$2,100,000$

Anaheim-Santa Ana-Garden Grove SHSA

Long Beach

$2,100,000$

Huntington Beach

390,000

180,000

Riverside

180,000

Pomona

110,000

$68 \mathrm{~km} \quad$ SSE

$62 \mathrm{~km} \quad \mathrm{NW}$

$75 \mathrm{~km} \quad \mathrm{NW}$

$61 \mathrm{~km} \quad \mathrm{NW}$

$68 \mathrm{~km} \quad \mathrm{~N}$

$79 \mathrm{~km} \quad \mathrm{NNW}$

SITE-SPECIFIC DATA - AIRBORNE PATHWAYS

Average Annual State Production

of Crops and Animal Products

In $80-\mathrm{km}$ Radius Circle

Regional Productivity Factor:

Animal Grazing Factor:
Veg: $4.8 E 7$ kilogram

Milk: 2.3E8 liter

Meat: $5.0 \mathrm{E} 7$ kilogram

0.6

1

Meteorology Period of Record: 25 JAN $73-24$ JAN 76 Recovery: $88 \%$

SITE-SPECIFIC DATA - WATERBORNE PATHWAYS VIa PACIFIC OCEAN

Average Dilution Flow

from Plant: $?, 700 \mathrm{ft}^{3} / \mathrm{s}$

Fish:

Edible Harvest: $2.9 \mathrm{E} 4 \mathrm{~kg} / \mathrm{yr}$

Dilution Factor: ${ }_{1}^{(a)}$

Invertebrates:

Edible Harvest: $2.9 \mathrm{E} 3 \mathrm{~kg} / \mathrm{yr}$
Dilution Factor: $1(\mathrm{a})$

(a) Seafood caught in undiluted effluent according to FES (1973). 
POPULATION DOSE-COMMITMENT ESTIMATES AND

AVERAGE INDIVIDUAL DOSE-COMMITMENT HISTOGRAM FOR

SAN ONOFRE 1,2 , AND 3

Dose Commitments (person-rem) from Waterborne Pathways

Iotal Body GI-LII $\underline{\text { Thyroid }}$ Bone $\underline{\text { Liver }}$

$\begin{array}{llllll}\text { Infant } & 0.0 \mathrm{E}+00 & 0.0 \mathrm{E}+00 & 0.0 \mathrm{E}+00 & 0.0 \mathrm{E}+00 & 0.0 \mathrm{E}+00 \\ \text { Child } & 3.3 \mathrm{E}-02 & 1.8 \mathrm{E}-01 & 3.7 \mathrm{E}-02 & 1.1 \mathrm{E}-01 & 1.2 \mathrm{E}-01 \\ \text { Teen } & 4.1 \mathrm{E}-02 & 3.7 \mathrm{E}-01 & 2.7 \mathrm{E}-02 & 6.6 \mathrm{E}-02 & 9.8 \mathrm{E}-02 \\ \text { Adult } & 3.8 \mathrm{E}-01 & 3.2 \mathrm{E}+00 & 1.8 \mathrm{E}-01 & 3.8 \mathrm{E}-02 & 5.8 \mathrm{E}-01 \\ \text { TOTAL } & 4.6 \mathrm{E}-01 & 3.8 \mathrm{E}+00 & 2.4 \mathrm{E}-01 & 5.6 \mathrm{E}-01 & 7.9 \mathrm{E}-01\end{array}$

Dose Commitments (person-rem) from Airborne Pathways

Total Body GI-LII $\underline{\text { Thrroid }}$ Bone Liver Lung

Infant $2.2 \mathrm{E}-02 \quad 2.1 \mathrm{E}-02 \quad 5.7 \mathrm{E}-01 \quad 2.3 \mathrm{E}-02 \quad 2.3 \mathrm{E}-02 \quad 2.3 \mathrm{E}-02$

Child 2.4E-01 2.4E-01 $3.2 \mathrm{E}+00 \quad 2.5 \mathrm{E}-01 \quad 2.5 \mathrm{E}-01 \quad 2.6 \mathrm{E}-01$

$\begin{array}{lllllll}\text { Teen } & 1.8 \mathrm{E}-01 & 1.7 \mathrm{E}-01 & 1.4 \mathrm{E}+00 & 1.8 \mathrm{E}-01 & 1.8 \mathrm{E}-01 & 2.1 \mathrm{E}-01\end{array}$

Adult $\quad 1.1 \mathrm{E}+00 \quad 1.1 \mathrm{E}+00 \quad 5.1 \mathrm{E}+00 \quad 1.1 \mathrm{E}+00 \quad 1.1 \mathrm{E}+00 \quad 1.2 \mathrm{E}+00$

$\begin{array}{lllllll}\text { TOTAL } & 1.5 \mathrm{E}+00 & 1.5 \mathrm{E}+00 & 1.0 \mathrm{E}+01 & 1.5 \mathrm{E}+00 & 1.5 \mathrm{E}+00 & 1.6 \mathrm{E}+00\end{array}$

Production/Consumption factors:

Produce: $0.028 \quad$ Milk: $0.20 \quad$ Meat: 0.072

Fraction of Population Recejving an Average Individual

Total-Body Dose Commitment from Airborne Pathways

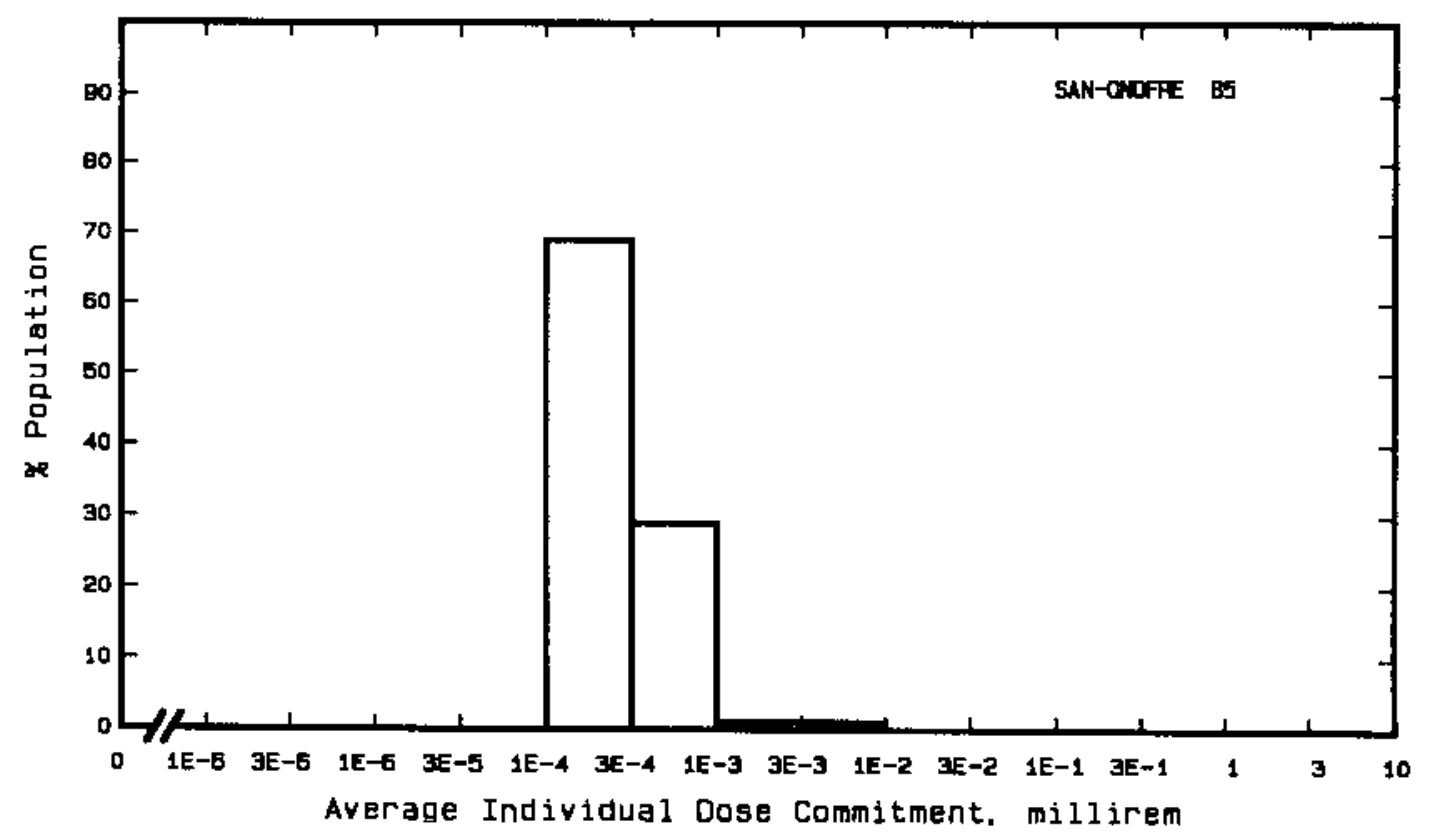


Site: SEQUOYAH

HAMILTON COUNTY, TENNESSEE

Location: $\quad$ N $35.2233^{\circ} \quad$ W $85.0878^{\circ}$

POPULATION DATA

Total Population Within 2-to-80-km Region: $8.6 \mathrm{E5}$

Major Metropolitan Centers Within Region:

Center

Chattanooga SMSA

Clevel and

East Ridge

Dalton

Athens
Population

430,000

26,000

21,000

21,000

12,000
Location

$28 \mathrm{~km} \quad \mathrm{SW}$

$21 \mathrm{~km} \quad \mathrm{SE}$

$27 \mathrm{~km} \quad \mathrm{SSW}$

$50 \mathrm{~km} \quad \mathrm{~S}$

$53 \mathrm{~km} \quad \mathrm{ENE}$

SITE-SPECIFIC DATA - AIRBORNE PATHWAYS

Average Annual State Production

of Crops and Animal Products

In 80-km Radius Circle

Regional Productivity Factor:

Animal Grazing Factor:

Meteorology Period of Record: 1 JAN 72 - 31 DEC 75 Recovery: $93 \%$
Veg: $1.1 \mathrm{E} 7 \mathrm{ki}$ logram

Milk: 1.6E8 liter

Meat: $1.2 E 8$ kilogram

0.25

0.7

SITE-SPECIFIC DATA - WATERBORNE PATHWAYS via TENNESSEE RIVER

Average River Flow

at Site: $35,000 \mathrm{ft}^{3} / \mathrm{s}$

Drinking Water:

Exposed Population: 450,000

Dilution Factor: 1

Fish:

Edible Harvest: $1.7 \mathrm{E5}{ }^{(\mathrm{a})} \mathrm{kg} / \mathrm{yr}$

Dilution Factor: 1

(a) Catch data given in FES (1974). 
POPULATION DOSE-COMMITMENT ESTIMATES AND

AVERAGE INDIVIDUAL DOSE-COMMITMENT HISTOGRAM FOR

SEQUOYAH 1 AND 2

Dose Commitments (person-rem) from Waterborne Pathways

Total Body GI-LII $\underline{\text { Thyroid }}$ Bone $\underline{\text { Liver }}$

$\begin{array}{llllll}\text { Infant } & 7.8 \mathrm{E}-03 & 7.2 \mathrm{E}-03 & 1.8 \mathrm{E}-02 & 4.9 \mathrm{E}-03 & 1.4 \mathrm{E}-02 \\ \text { Child } & 1.0 \mathrm{E}-01 & 8.6 \mathrm{E}-02 & 1.5 \mathrm{E}-01 & 1.1 \mathrm{E}-01 & 2.1 \mathrm{E}-01 \\ \text { Teen } & 6.3 \mathrm{E}-02 & 4.0 \mathrm{E}-02 & 5.3 \mathrm{E}-02 & 4.6 \mathrm{E}-02 & 1.1 \mathrm{E}-01 \\ \text { Adult } & 6.2 \mathrm{E}-01 & 3.5 \mathrm{E}-01 & 4.2 \mathrm{E}-01 & 2.7 \mathrm{E}-01 & 7.4 \mathrm{E}-01 \\ \text { TOTAL } & 7.9 \mathrm{E}-01 & 4.8 \mathrm{E}-01 & 6.4 \mathrm{E}-01 & 4.3 \mathrm{E}-01 & 1.1 \mathrm{E}+00\end{array}$

Dose Commitments (person-rem) from Airborne Pathways

Total Body GI-LII Thyroid Bone Liver Lung

$\begin{array}{lllllll}\text { Infant } & 3.6 \mathrm{E}-03 & 3.6 \mathrm{E}-03 & 4.2 \mathrm{E}-03 & 3.1 \mathrm{E}-03 & 3.6 \mathrm{E}-03 & 3.9 \mathrm{E}-03 \\ \text { Chi1d } & 4.3 \mathrm{E}-02 & 4.3 \mathrm{E}-02 & 4.8 \mathrm{E}-02 & 3.4 \mathrm{E}-02 & 4.3 \mathrm{E}-02 & 4.7 \mathrm{E}-02 \\ \text { Teen } & 3.2 \mathrm{E}-02 & 3.2 \mathrm{E}-02 & 3.5 \mathrm{E}-02 & 2.5 \mathrm{E}-02 & 3.2 \mathrm{E}-02 & 3.7 \mathrm{E}-02 \\ \text { Adult } & 1.9 \mathrm{E}-01 & 1.9 \mathrm{E}-01 & 2.0 \mathrm{E}-01 & 1.5 \mathrm{E}-01 & 1.9 \mathrm{E}-01 & 2.1 \mathrm{E}-01 \\ \text { TOTAL } & 2.7 \mathrm{E}-01 & 2.7 \mathrm{E}-01 & 2.9 \mathrm{E}-01 & 2.1 \mathrm{E}-01 & 2.7 \mathrm{E}-01 & 3.0 \mathrm{E}-01\end{array}$

Production/Consumption factors:

Produce: 0.016

Mijk: $\quad 0.37$

Meat: 0.44

Fraction of Population Receiving an Average Individual

Total-Body Dose Commitment from Airborne Pathways

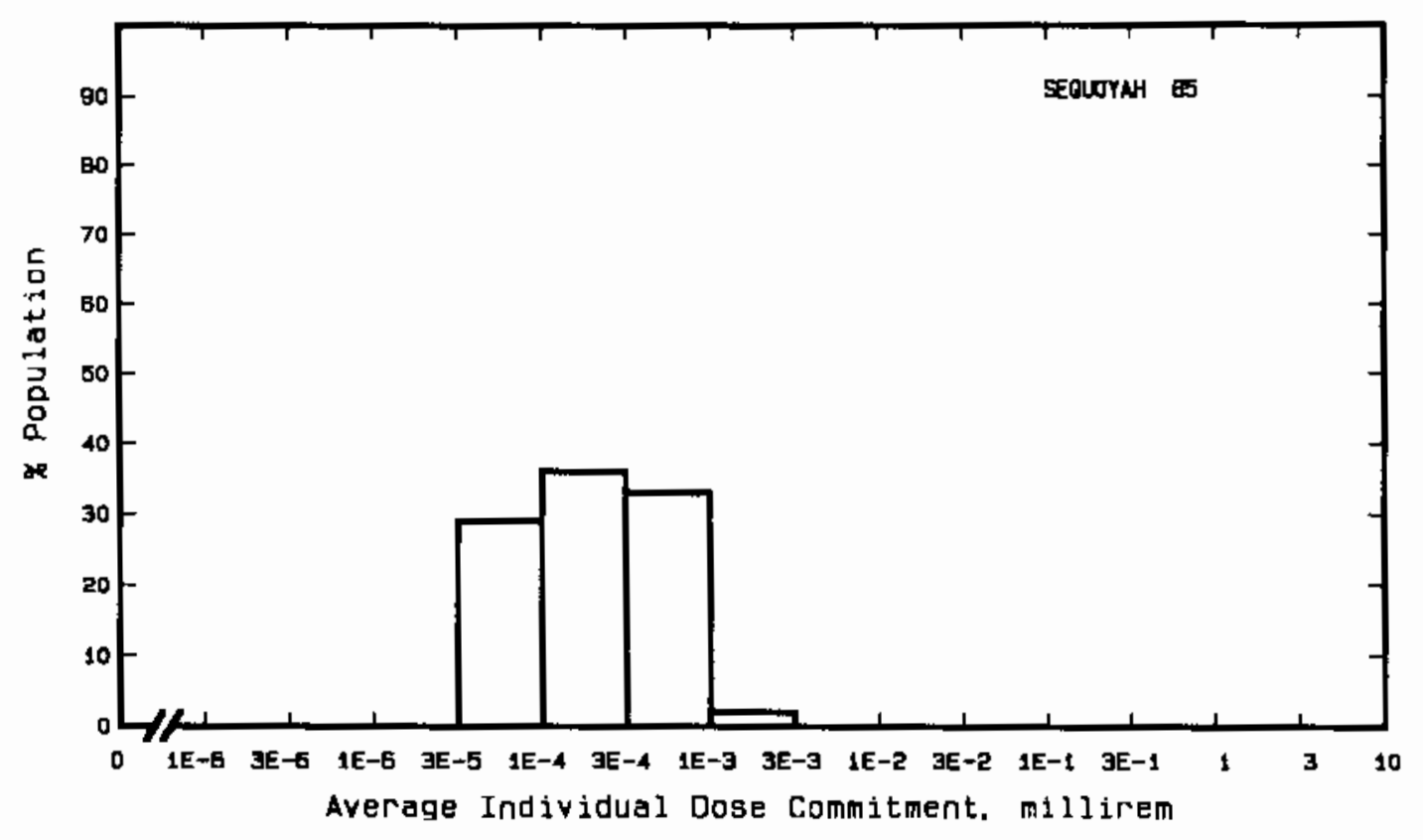


Site: SUMMER

JENKINSVILLE, SOUTH CAROLINA

Location: N $\quad$ N $34.2958^{\circ} \quad$ W $81.3203^{\circ}$

POPULATION DATA

Total Population Within 2-to-80-km Region: 8.7E5

Major Metropolitan Centers Within Region:

Center
Columbia SMSA
Rock Hill
Greenwood
Union
Laurens

Population

Location

Columbia SMSA

440,000

36,000

23,000

11,000

$42 \mathrm{~km} \quad \mathrm{SE}$

$75 \mathrm{~km} \quad$ NNE

$78 \mathrm{~km} \mathrm{~W}$

$54 \mathrm{~km} \quad \mathrm{NNW}$

$68 \mathrm{~km} \quad W N W$

\section{SITE-SPECIFIC DATA - AIRBORNE PATHWAYS}

Average Annual State Production

of Crops and Animal Products

In $80-\mathrm{km}$ Radius Circle

Regional Productivity Factor:

Animal Grazing Factor:
Veg: $7.4 \mathrm{E} 6 \mathrm{kilogram}$

Milk: $5.7 E 7$ Titer

Meat: 5.0E7 kilogram

Meteorology Period of Record: 1 Jan 75 - 31 Dec 75 Recovery: $98 \%$

SITE-SPECIFIC DATA - WATERBORNE PATHWAYS via PARR RES, and BROAD RIVER

Average River $\mathrm{Flow}$

at Site: $6,000 \mathrm{ft}^{3} / \mathrm{s}$

Drinking Water:

Exposed Population: 130,000

Dilution Factor: 1

Fish:

Edible Harvest: (a)

Dilution Factor: 1

(a) Average individual consumption rate of $2.2 \mathrm{~kg} / \mathrm{yr}$ as given in the FES (1973) used in lieu of catch data. 
POPULATION DOSE-COMMITMENT ESTIMATES AND AVERAGE INDIVIDUAL DOSE-COMMITMENT HISTOGRAM FOR

\section{SUMMER}

Dose Comitments (person-rem) from Waterborne Pathways

Total Body GI-LII $\underline{\text { Thyroid }}$ Bone Liver

$\begin{array}{llllll}\text { Infant } & 6.1 \mathrm{E}-03 & 6.1 \mathrm{E}-03 & 8.7 \mathrm{E}-03 & 1.7 \mathrm{E}-04 & 6.0 \mathrm{E}-03 \\ \text { Child } & 8.4 \mathrm{E}-02 & 1.7 \mathrm{E}-01 & 9.0 \mathrm{E}-02 & 3.3 \mathrm{E}-02 & 1.2 \mathrm{E}-01 \\ \text { Teen } & 4.7 \mathrm{E}-02 & 2.3 \mathrm{E}-01 & 3.4 \mathrm{E}-02 & 2.0 \mathrm{E}-02 & 7.0 \mathrm{E}-02 \\ \text { Adult } & 4.2 \mathrm{E}-01 & 2.0 \mathrm{E}+00 & 2.8 \mathrm{E}-01 & 1.2 \mathrm{E}-01 & 4.8 \mathrm{E}-01 \\ \text { TOTAL } & 5.6 \mathrm{E}-01 & 2.4 \mathrm{E}+00 & 4.1 \mathrm{E}-01 & 1.7 \mathrm{E}-01 & 6.8 \mathrm{E}-01\end{array}$

Dose Commitments (person-rem) from Airborne Pathways

Iotal Body GI-LII Ihrroid Bone Liver Lung

$\begin{array}{lllllll}\text { Infant } & 3.5 \mathrm{E}-05 & 3.5 \mathrm{E}-05 & 4.1 \mathrm{E}-05 & 3.5 \mathrm{E}-05 & 3.5 \mathrm{E}-05 & 3.8 \mathrm{E}-05 \\ \text { Child } & 4.0 \mathrm{E}-04 & 4.0 \mathrm{E}-04 & 4.3 \mathrm{E}-04 & 3.8 \mathrm{E}-04 & 4.0 \mathrm{E}-04 & 4.4 \mathrm{E}-04 \\ \text { Teen } & 2.9 \mathrm{E}-04 & 2.9 \mathrm{E}-04 & 3.0 \mathrm{E}-04 & 2.8 \mathrm{E}-04 & 2.9 \mathrm{E}-04 & 3.5 \mathrm{E}-04 \\ \text { Adult } & 1.8 \mathrm{E}-03 & 1.8 \mathrm{E}-03 & 1.8 \mathrm{E}-03 & 1.7 \mathrm{E}-03 & 1.8 \mathrm{E}-03 & 1.9 \mathrm{E}-03 \\ \text { TOTAL } & 2.5 \mathrm{E}-03 & 2.5 \mathrm{E}-03 & 2.6 \mathrm{E}-03 & 2.4 \mathrm{E}-03 & 2.5 \mathrm{E}-03 & 2.8 \mathrm{E}-03\end{array}$

Production/Consumption factors:

Produce: 0.039

Milk: $0.45 \quad$ Meat: 0.64

Fraction of Population Receiving an Average Individual Tota1-Body Dose Commitment from Airborne Pathways

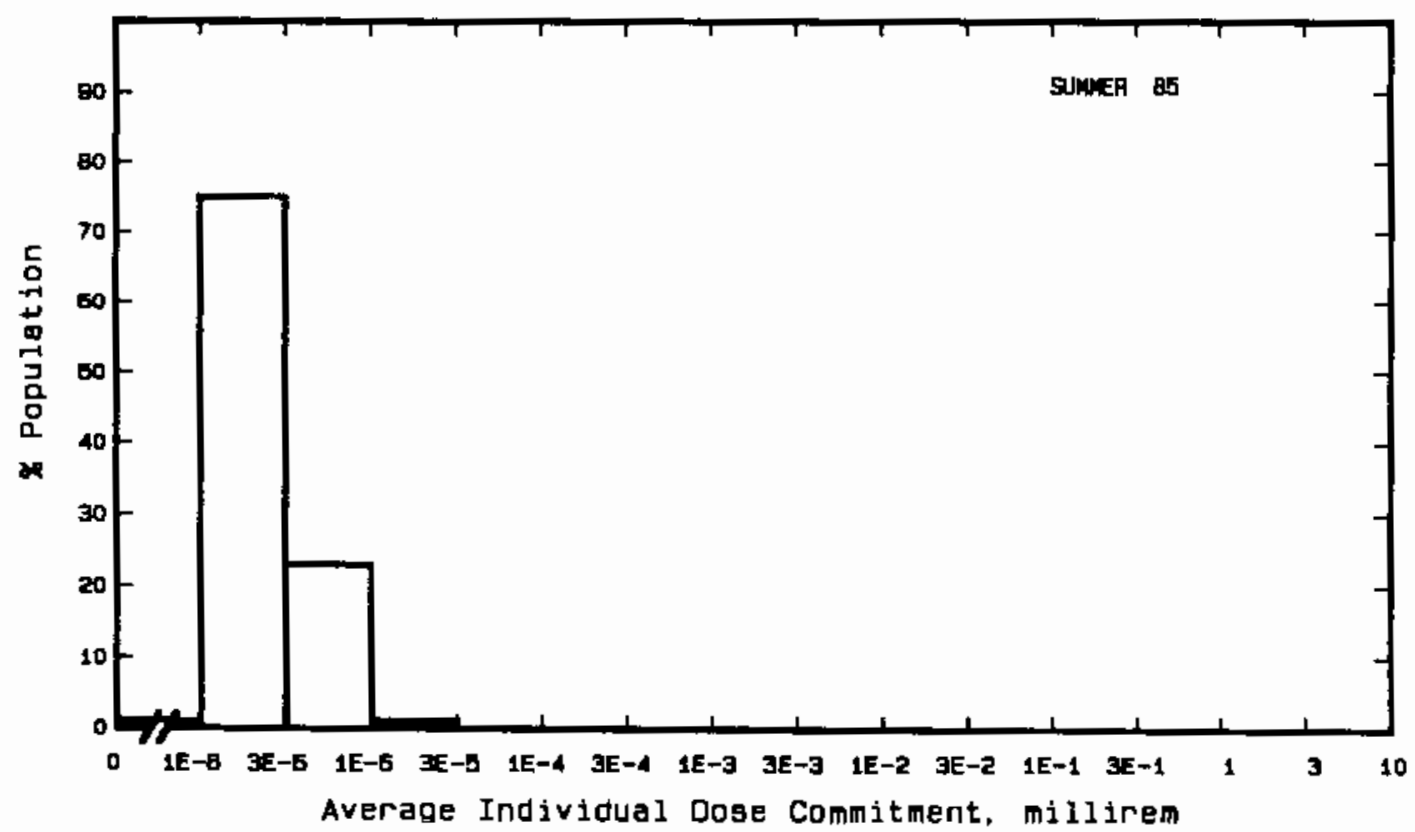


Site: SURRY

SURRY COUNTY, VIRGINIA

Location: N $37.1656^{\circ}$ W $76.6983^{\circ}$

POPULATION DATA

Total Population Within 2-to-80-km Region: J.8E6

Major Metropolitan Centers Within Region:

Center

Norfolk-Virginia Beach-Portsmouth SMSA

Richmond SMSA

Newport News-Hampton SMSA

Petersburg-Colonial Heights-Hopewell SMSA

Willi amsburg
Population

820,000

650,000

370,000

130,000

10,000
Location

$50 \mathrm{~km} \quad \mathrm{SE}$

$77 \mathrm{~km} \quad W N W$

$33 \mathrm{~km} \quad \mathrm{ESE}$

$63 \mathrm{~km} \mathrm{~W}$

$12 \mathrm{~km} \mathrm{~N}$

\section{SITE-SPECIFIC DATA - AIRBORNE PATHWAYS}

Average Annual State Production

of Crops and Animal Products

In $80-\mathrm{km}$ Radius Circle

Regional Productivity Factor:

Animal Grazing Factor:
Veg: $3.5 E 7$ kilogram

Milk: 1.5E8 liter

Meat: 7.4E7 kilogram

0.8

0.7

Meteorology Period of Record: 3 MAR 74 - 2 MAR 75 Recovery: $91 \%$

SITE-SPECIFIC DATA - WATERBORNE PATHWAYS via JAMES RIVER ESTUARY

Average River Flow

at Site: $25,000 \mathrm{ft}^{3} / \mathrm{s}(\mathrm{a})$

Fish:

Edible Harvest: $6.0 \mathrm{E} 5 \mathrm{~kg} / \mathrm{yr}$

Dilution Factor: 1

Invertebrates: (b)

Edible Harvest: $1.1 \mathrm{E} 6 \mathrm{~kg} / \mathrm{yr}$

Dilution Factor: 1

(a) Flow includes fresh water river flow and saline "mixing flow" of estuary as given in FES (1972).

(b) Environment primarily salt water so invertebrates considered in lieu of drinking water. 
POPULATION DOSE-COMMITMENT ESTIMATES AND

AVERAGE INDIVIDUAL DOSE-COMMITMENT HISTOGRAM FOR

SURRY 1 AND 2

Dose Commitments (person-rem) from Waterborne Pathways

Total Body GI-LII $\underline{\text { Thyroid }}$ Bone Liver

$\begin{array}{llllll}\text { Infant } & 0.0 \mathrm{E}+00 & 0.0 \mathrm{E}+00 & 0.0 \mathrm{E}+00 & 0.0 \mathrm{E}+00 & 0.0 \mathrm{E}+00 \\ \text { Child } & 2.1 \mathrm{E}-01 & 3.6 \mathrm{E}-01 & 2.7 \mathrm{E}-01 & 4.0 \mathrm{E}-01 & 3.4 \mathrm{E}-01 \\ \text { Teen } & 1.6 \mathrm{E}-01 & 7.3 \mathrm{E}-01 & 1.9 \mathrm{E}-01 & 2.3 \mathrm{E}-01 & 2.7 \mathrm{E}-01 \\ \text { Adult } & 1.1 \mathrm{E}+00 & 6.4 \mathrm{E}+00 & 1.2 \mathrm{E}+00 & 1.3 \mathrm{E}+00 & 1.6 \mathrm{E}+00 \\ \text { TOTAL } & 1.5 \mathrm{E}+00 & 7.5 \mathrm{E}+00 & 1.7 \mathrm{E}+00 & 1.9 \mathrm{E}+00 & 2.2 \mathrm{E}+00\end{array}$

Dose Commitments (person-rem) from Airborne Pathways

Total Body GI-LII Thyroid Bone Liver Lung

$\begin{array}{lllllll}\text { Infant } & 1.4 \mathrm{E}-03 & 1.4 \mathrm{E}-03 & 1.9 \mathrm{E}-02 & 1.2 \mathrm{E}-03 & 1.5 \mathrm{E}-03 & 1.5 \mathrm{E}-03 \\ \text { Child } & 1.7 \mathrm{E}-02 & 1.6 \mathrm{E}-02 & 1.2 \mathrm{E}-01 & 1.3 \mathrm{E}-02 & 1.7 \mathrm{E}-02 & 1.8 \mathrm{E}-02 \\ \text { Teen } & 1.2 \mathrm{E}-02 & 1.2 \mathrm{E}-02 & 5.7 \mathrm{E}-02 & 9.5 \mathrm{E}-03 & 1.2 \mathrm{E}-02 & 1.4 \mathrm{E}-02 \\ \text { Adult } & 7.1 \mathrm{E}-02 & 7.1 \mathrm{E}-02 & 2.4 \mathrm{E}-01 & 5.7 \mathrm{E}-02 & 7.2 \mathrm{E}-02 & 7.7 \mathrm{E}-02 \\ \text { TOTAL } & 1.0 \mathrm{E}-01 & 1.0 \mathrm{E}-01 & 4.4 \mathrm{E}-01 & 8.1 \mathrm{E}-02 & 1.0 \mathrm{E}-01 & 1.1 \mathrm{E}-01\end{array}$

Production/Consumption factors:

Produce: $0.080 \quad$ Milk: $0.51 \quad$ Meat: 0.41

Fraction of Population Receiving an Average Individual

Total-Body Dose Commitment from Airborne Pathways

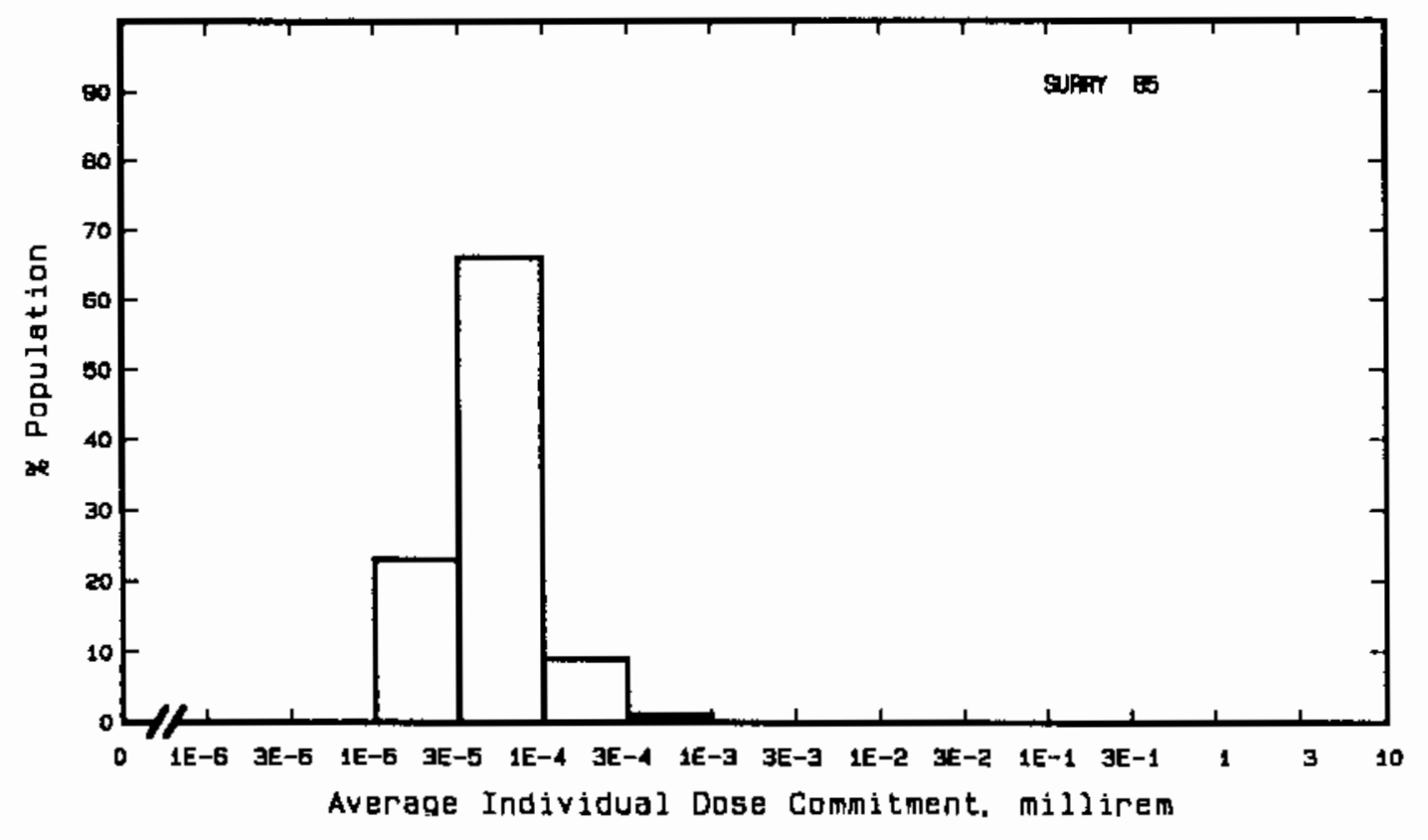


Site: SUSQUEHANNA

BERWICK, PENNSYLVANIA

Location: $\quad$ N $41.1000^{\circ}$ W $76.1500^{\circ}$

POPULATION DATA

Total Population Within 2-to-80-km Region: 1.5E6

Major Metropolitan Centers Within Region:

Center

Williamsport SMSA

Allentown

Scranton

Wilkes-Barre

Hazleton
Population

118,000 100,000

88,000

52,000

27,000
Location

$73 \mathrm{~km}$ WNW

$79 \mathrm{~km} \quad \mathrm{SE}$

$53 \mathrm{~km} \quad \mathrm{NE}$

$28 \mathrm{~km} \quad \mathrm{NE}$

$21 \mathrm{~km} \quad \mathrm{SE}$

SITE-SPECIFIC DATA - AIRBORNE PATHWAYS

Average Annual State Production

of Crops and Animal Products

In $80-\mathrm{km}$ Radius Circle

Regional Productivity Factor:

Animal Grazing Factor:
Veg: $\quad 5.3 \mathrm{E} 7$ kilogram

Milk: $5.3 \mathrm{E} 8$ liter

Meat: $5.4 E 7$ kilogram

0.9

0.6

Meteorology Period of Record: 1 Jan 75 - 31 Dec 75 Recovery: $98 \%$

Average River Flow

at Site: $13,1000 \mathrm{ft}^{3} / \mathrm{s}$

Drinking Water:

Exposed Population: 100,000

Dilution Factor: 1

Fish:

Edible Harvest: (a)

Dilution Factor: $0.1^{\text {(b) }}$

(a) No fish catch data given in FES (1973), so generic consumption rates used (Table A.1).

(b) Ten percent of population consumes fish from river. 
POPULATION DOSE-COMMITMENT ESTIMATES AND

AVERAGE INDIVIDUAL DOSE-COMMITMENT HISTOGRAM FOR

SUSQUEHANNA 1 AND 2

Dose Commitments (person-rem) from Waterborne Pathways

Total Body GI-LII Thyroid Bone Liver

$\begin{array}{llllll}\text { Infant } & 8.6 \mathrm{E}-05 & 1.0 \mathrm{E}-04 & 1.2 \mathrm{E}-04 & 1.0 \mathrm{E}-05 & 9.9 \mathrm{E}-05 \\ \text { Child } & 2.1 \mathrm{E}-03 & 2.7 \mathrm{E}-03 & 1.2 \mathrm{E}-03 & 6.5 \mathrm{E}-04 & 3.1 \mathrm{E}-03 \\ \text { Teen } & 1.2 \mathrm{E}-03 & 3.8 \mathrm{E}-03 & 4.4 \mathrm{E}-04 & 4.2 \mathrm{E}-04 & 2.4 \mathrm{E}-03 \\ \text { Adult } & 7.6 \mathrm{E}-03 & 3.5 \mathrm{E}-02 & 3.4 \mathrm{E}-03 & 2.8 \mathrm{E}-03 & 1.5 \mathrm{E}-02 \\ \text { TOTAL } & 1.1 \mathrm{E}-02 & 4.1 \mathrm{E}-02 & 5.2 \mathrm{E}-03 & 3.9 \mathrm{E}-03 & 2.1 \mathrm{E}-02\end{array}$

Dose Commitments (person-rem) from Airborne Pathways

Total Body GI-LII Thyroid Bone Liver Lung

$\begin{array}{lllllll}\text { Infant } & 1.3 \mathrm{E}-03 & 1.3 \mathrm{E}-03 & 2.9 \mathrm{E}-03 & 6.6 \mathrm{E}-04 & 1.4 \mathrm{E}-03 & 1.4 \mathrm{E}-03 \\ \text { Child } & 1.7 \mathrm{E}-02 & 1.7 \mathrm{E}-02 & 2.6 \mathrm{E}-02 & 7.3 \mathrm{E}-03 & 1.7 \mathrm{E}-02 & 1.7 \mathrm{E}-02 \\ \text { Teen } & 1.1 \mathrm{E}-02 & 1.2 \mathrm{E}-02 & 1.5 \mathrm{E}-02 & 5.3 \mathrm{E}-03 & 1.2 \mathrm{E}-02 & 1.2 \mathrm{E}-02 \\ \text { Adult } & 6.5 \mathrm{E}-02 & 6.6 \mathrm{E}-02 & 7.7 \mathrm{E}-02 & 3.2 \mathrm{E}-02 & 6.5 \mathrm{E}-02 & 6.8 \mathrm{E}-02 \\ \text { TOTAL } & 9.5 \mathrm{E}-02 & 9.6 \mathrm{E}-02 & 1.2 \mathrm{E}-01 & 4.5 \mathrm{E}-02 & 9.5 \mathrm{E}-02 & 9.9 \mathrm{E}-02\end{array}$

Production/Consumption factors:

Produce: 0.17

Milk: 2.4

Meat: 0.41

Fraction of Population Receiving an Average Individual Tota1-Body Dose Commitment from Airborne Pathways

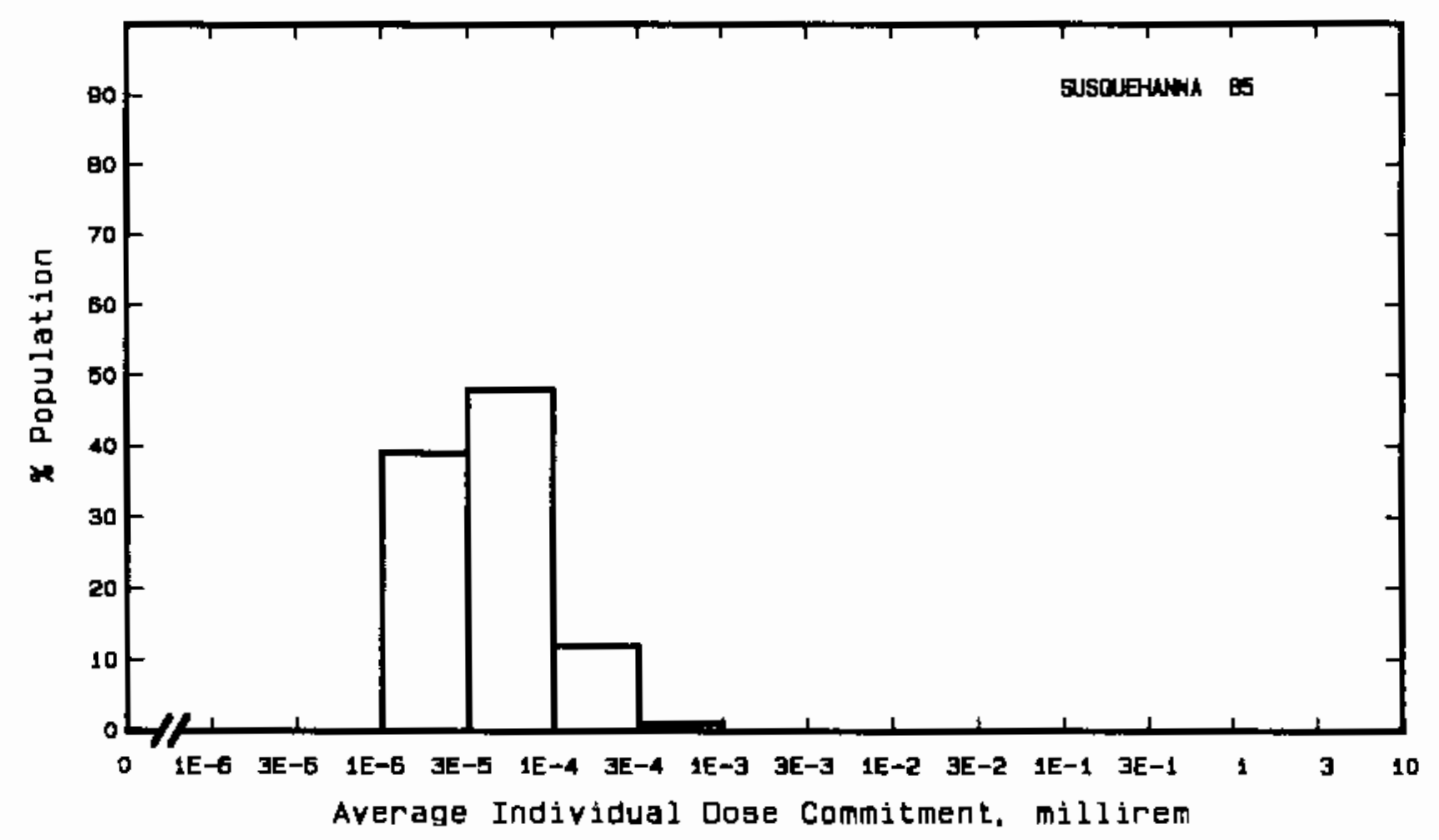


Site: THREE MILE ISLAND

THREE MILE ISLAND, PENNSYLVANia

Location: N $40.1531^{\circ}$ W $76.7250^{\circ}$

PDPULATION DATA

Total Population Within 2-to-80-km Region: 2.1E6

Major Metropolitan Centers Within Region:

Center

Population

Location

Harrisburg SMSA

York SMSA

460,000

Lancaster SMSA

390,000

Reading SMSA

390,000

320,000

Lebanon

26,000

$18 \mathrm{~km} \quad \mathrm{NW}$

$21 \mathrm{~km} \quad \mathrm{~S}$

$38 \mathrm{~km} \quad$ ESE

$71 \mathrm{~km} \quad$ ENE

$33 \mathrm{~km} \quad \mathrm{NE}$

SITE-SPECIFIC DATA - AIRBORNE PATHWAYS

Average Annual State Production

of Crops and Animal Products

In $80-\mathrm{km}$ Radius Circle

Regional Productivity Factor:

Animal Grazing Factor:
Veg: $5.3 E 7 \mathrm{kilogram}$

Milk: 5.3E8 liter

Meat: $5.4 E 7$ kilogram

1

0.5

Meteorology Period of Record: 1 OCT 72 - 30 SEP 73 Recovery: $80 \%$

SITE-SPECIFIC DATA - WATERBORNE PATHWAYS via SUSQUEHANNA RIVER

Average River Flow at Site: $34,000 \mathrm{ft}^{3} / \mathrm{s}$

Drinking Water:

Exposed Population: 230,000

Dilution Factor: 1

Fish:

Edible Harvest: (a)

Dilution Factor: $0.025^{\text {(b) }}$

(a) No fish catch data given in FES (1972), so generic consumption rates used (Table A-1).

(b) Ten percent of population consumes $25 \%$ of their fish from river according to FES. 
POPULATION DOSE-COMMITMENT ESTIMATES AND

AVERAGE INDIVIDUAL DOSE-COMMITMENT HISTOGRAM FOR

THREE MILE ISLAND 1 AND 2

Dose Commitments (person-rem) from Waterborne Pathways(a)

Total Body GI-LII Thyroid Bone Liver

$\begin{array}{llllll}\text { Infant } & 7.7 \mathrm{E}-05 & 5.3 \mathrm{E}-05 & 5.2 \mathrm{E}-05 & 1.3 \mathrm{E}-04 & 1.1 \mathrm{E}-04 \\ \text { Child } & 1.3 \mathrm{E}-03 & 6.2 \mathrm{E}-04 & 5.8 \mathrm{E}-04 & 3.8 \mathrm{E}-03 & 3.0 \mathrm{E}-03 \\ \text { Teen } & 9.7 \mathrm{E}-04 & 2.7 \mathrm{E}-04 & 2.2 \mathrm{E}-04 & 1.8 \mathrm{E}-03 & 1.9 \mathrm{E}-03 \\ \text { AduTt } & 9.7 \mathrm{E}-03 & 2.3 \mathrm{E}-03 & 1.9 \mathrm{E}-03 & 1.1 \mathrm{E}-02 & 1.2 \mathrm{E}-02 \\ \text { TOTAL } & 1.2 \mathrm{E}-02 & 3.2 \mathrm{E}-03 & 2.7 \mathrm{E}-03 & 1.7 \mathrm{E}-02 & 1.7 \mathrm{E}-02\end{array}$

Dose Commitments (person-rem) from Airborne Pathways (a)

Total Body GI-LII Thyroid Bone Liver Lung

$\begin{array}{lllllll}\text { Infant } & 6.1 \mathrm{E}-04 & 6.1 \mathrm{E}-04 & 6.1 \mathrm{E}-04 & 2.1 \mathrm{E}-04 & 6.1 \mathrm{E}-04 & 6.2 \mathrm{E}-04 \\ \text { Child } & 8.0 \mathrm{E}-03 & 8.0 \mathrm{E}-03 & 8.0 \mathrm{E}-03 & 2.6 \mathrm{E}-03 & 8.0 \mathrm{E}-03 & 8.2 \mathrm{E}-03 \\ \text { Teen } & 5.5 \mathrm{E}-03 & 5.5 \mathrm{E}-03 & 5.5 \mathrm{E}-03 & 1.8 \mathrm{E}-03 & 5.5 \mathrm{E}-03 & 5.8 \mathrm{E}-03 \\ \text { Adult } & 3.1 \mathrm{E}-02 & 3.1 \mathrm{E}-02 & 3.1 \mathrm{E}-02 & 1.1 \mathrm{E}-02 & 3.1 \mathrm{E}-02 & 3.2 \mathrm{E}-02 \\ \text { TOTAL } & 4.5 \mathrm{E}-02 & 4.5 \mathrm{E}-02 & 4.5 \mathrm{E}-02 & 1.6 \mathrm{E}-02 & 4.5 \mathrm{E}-02 & 4.7 \mathrm{E}-02\end{array}$

Production/Consumption factors:

Produce: 0.13

Milk: 1.9

Meat: 0.32

Fraction of Population Receiving an Average Individual

Totel-Body Dose Commitment from Airborne Pathways

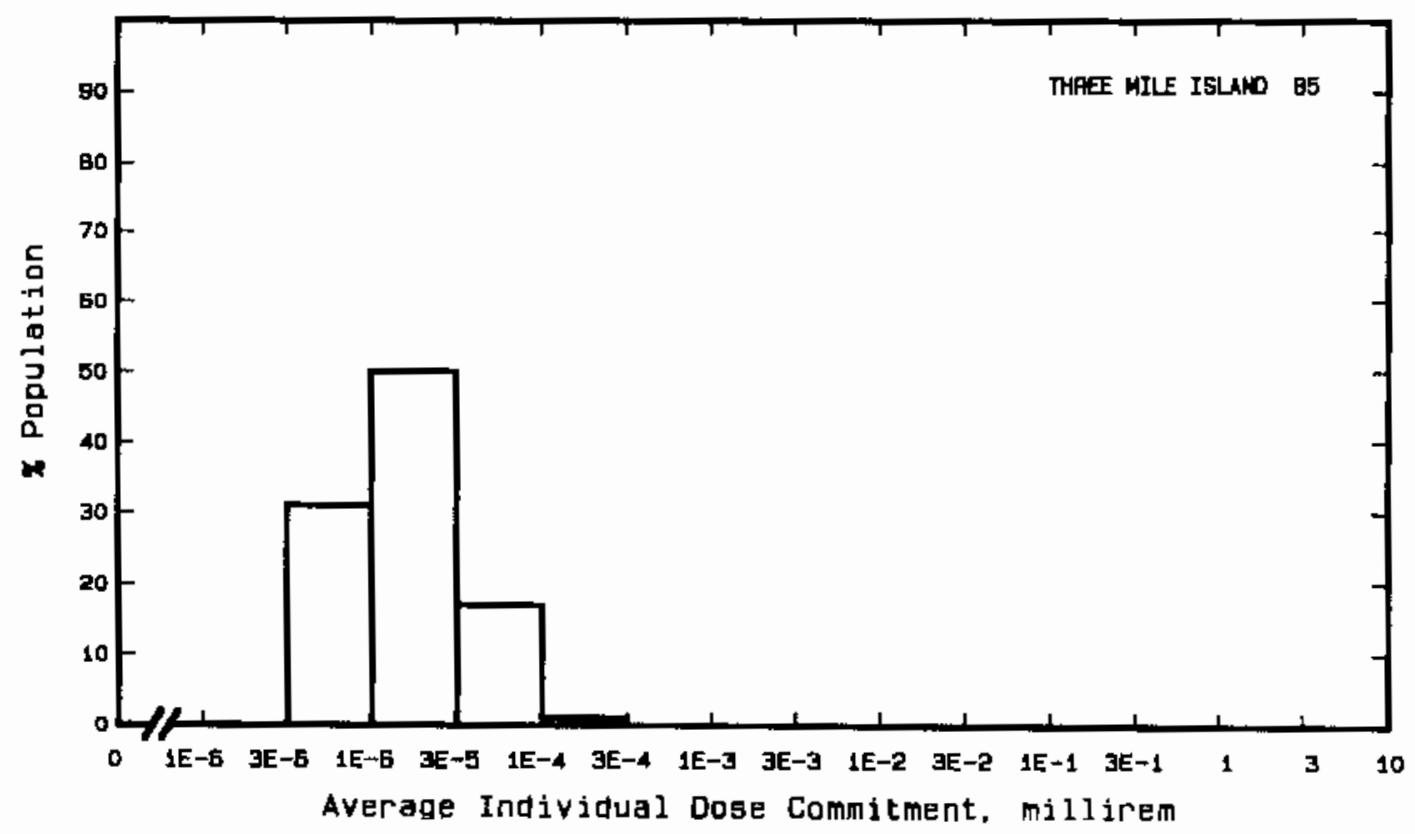

(a) Doses include releases from the TMI 2 / Epicore. 
Site: TROJAN

PRESCOTT, OREGON

Location: $\quad$ N $46.0408^{\circ} \quad$ W $122.8844^{\circ}$

POPULATION DATA

Total Population Within 2-to-80-km Region: 1.4E6

Major Metropolitan Centers Within Region:

Center Population Location

Portland-Vancouver SMSA $\quad 1,400,000 \quad 60 \mathrm{~km} \quad$ SSE

Longview

Astoria

33,000

$12 \mathrm{~km} \quad \mathrm{NNW}$

Forest Grove

14,000

$72 \mathrm{~km} \quad W N W$

Centralia

13,000

$58 \mathrm{~km} \quad$ SSW

12,000

$75 \mathrm{~km} \mathrm{~N}$

SITE-SPECIFIC DATA - AIRBORNE PATHWAYS

Average Annual State Production

of Crops and Animal Products

In $80-\mathrm{km}$ Radius Circle

Regional Productivity Factor:

Animal Grazing Factor:
Veg: $6.4 E 7 \mathrm{kilogram}$

Milk: 3.7E7 liter

Meat: $2.6 E 7$ kilogram

0.9

0.75

Meteorology Period of Record: 1 SEP 71 - 31 AUG 74 Recovery: 90\%

SITE-SPECIFIC DATA - WATERBORNE PATHWAYS VII COLUMBIA RIVER

Average River Flow

at Site: $230,000 \mathrm{ft}^{3} / \mathrm{s}$

Drinking Water:

Exposed Population: 540 (a)

Dilution Factor: 1

Fish:

Edible Harvest: $1.0 \mathrm{E} 6 \mathrm{~kg} / \mathrm{yr}$

Dilution Factor: 1

(a) Population of Rainier divided by 4, since residents only there for $25 \%$ of the year. 
POPULATION DOSE-COMMITMENT ESTIMATES AND

AVERAGE INDIVIDUAL DOSE-COMMITMENT HISTOGRAM FOR

TROJAN

Dose Commitments (person-rem) from Waterborne Pathways

Total Body GI-LII Thyroid Bone Liver

$\begin{array}{llllll}\text { Infant } & 5.7 \mathrm{E}-07 & 6.2 \mathrm{E}-07 & 2.4 \mathrm{E}-06 & 1.9 \mathrm{E}-07 & 7.4 \mathrm{E}-07 \\ \text { Child } & 1.7 \mathrm{E}-03 & 9.2 \mathrm{E}-04 & 3.3 \mathrm{E}-04 & 7.8 \mathrm{E}-03 & 9.4 \mathrm{E}-03 \\ \text { Teen } & 3.3 \mathrm{E}-03 & 1.9 \mathrm{E}-03 & 2.3 \mathrm{E}-04 & 4.7 \mathrm{E}-03 & 8.0 \mathrm{E}-03 \\ \text { Adult } & 3.5 \mathrm{E}-02 & 1.6 \mathrm{E}-02 & 1.6 \mathrm{E}-03 & 2.7 \mathrm{E}-02 & 4.8 \mathrm{E}-02 \\ \text { TOTAL } & 4.1 \mathrm{E}-02 & 1.9 \mathrm{E}-02 & 2.1 \mathrm{E}-03 & 4.0 \mathrm{E}-02 & 6.5 \mathrm{E}-02\end{array}$

Dose Commitments (person-rem) from Airborne Pathways

Iotal Body GI-LII Thyroid Bone Liver Lung

$\begin{array}{lllllll}\text { Infant } & 7.0 \mathrm{E}-04 & 7.0 \mathrm{E}-04 & 1.9 \mathrm{E}-03 & 6.4 \mathrm{E}-04 & 7.1 \mathrm{E}-04 & 7.6 \mathrm{E}-04 \\ \text { Child } & 9.0 \mathrm{E}-03 & 8.7 \mathrm{E}-03 & 2.0 \mathrm{E}-02 & 8.5 \mathrm{E}-03 & 8.8 \mathrm{E}-03 & 9.5 \mathrm{E}-03 \\ \text { Teen } & 6.5 \mathrm{E}-03 & 6.4 \mathrm{E}-03 & 1.2 \mathrm{E}-02 & 5.9 \mathrm{E}-03 & 6.4 \mathrm{E}-03 & 7.3 \mathrm{E}-03 \\ \text { Adu7t } & 3.9 \mathrm{E}-02 & 3.8 \mathrm{E}-02 & 6.1 \mathrm{E}-02 & 3.5 \mathrm{E}-02 & 3.8 \mathrm{E}-02 & 4.1 \mathrm{E}-02 \\ \text { TOTAL } & 5.5 \mathrm{E}-02 & 5.4 \mathrm{E}-02 & 9.5 \mathrm{E}-02 & 5.0 \mathrm{E}-02 & 5.4 \mathrm{E}-02 & 5.9 \mathrm{E}-02\end{array}$

Production/Consumption factors:

Produce: 0.21

Milk: 0.18

Meat: $\quad 0.20$

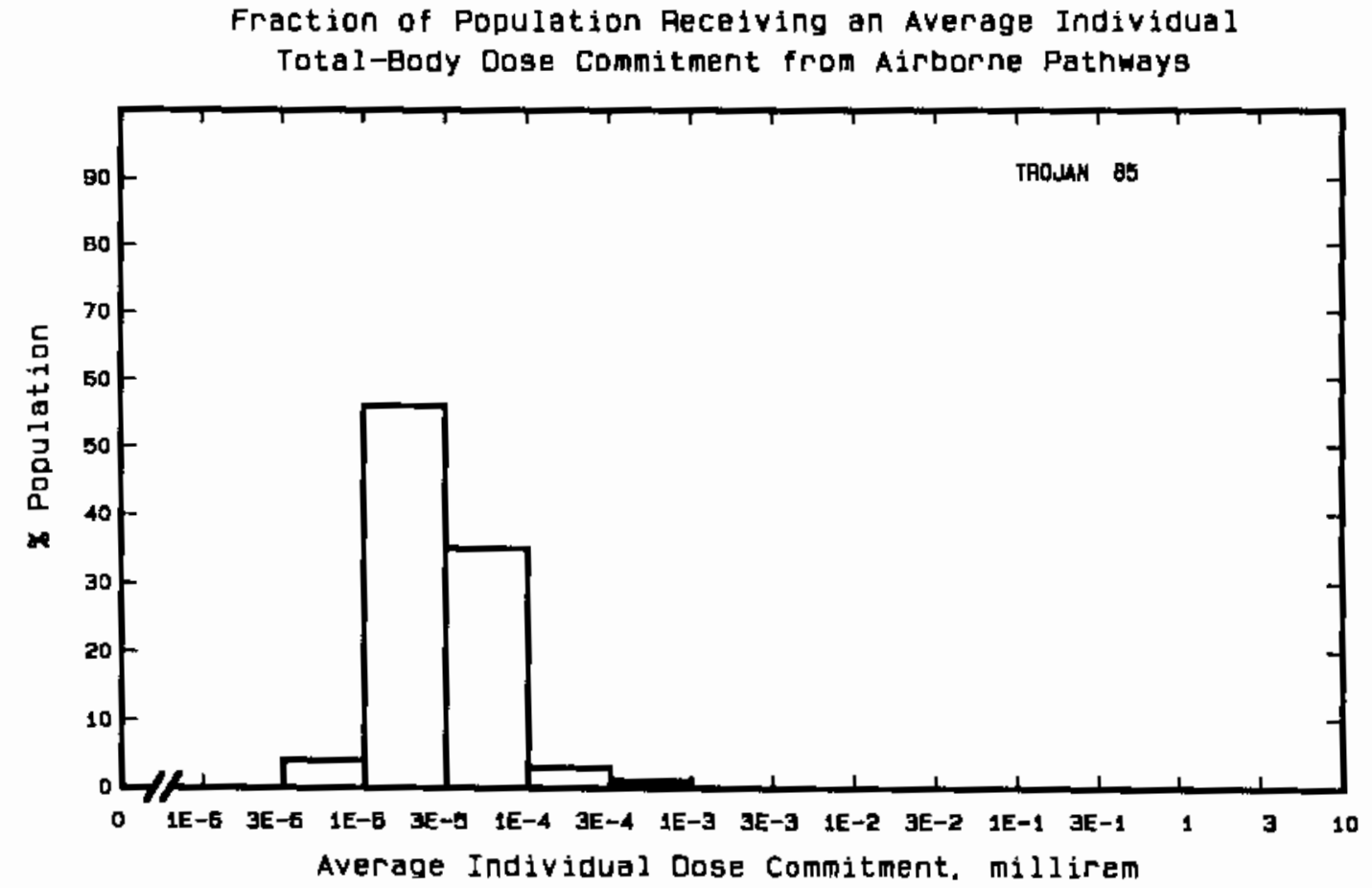


Site: TURKEY POINT

DADE COUIYTY, FLORIDA

Location: $\quad$ N $25.4350^{\circ} \quad$ W $80.3314^{\circ}$

POPULATION DATA

Total Population Within 2-to-80-km Region: 2.7E6

Major Metropolitan Centers Within Region:

Center

Miami-Hialeah SMSA

Fort Lauderdale-Hollywood SMSA Homestead

Upper Keys Division
Population

$1,800,000$

$1,100,000$

22,000

16,000

\section{Location}

$41 \mathrm{~km} \quad \mathrm{NNE}$

$79 \mathrm{~km} \quad \mathrm{NNE}$

$16 \mathrm{~km} \quad W$

$42 \mathrm{~km} \quad \mathrm{~S}$

SITE-SPECIFIC DATA - AIRBORNE PATHWAYS

Average Annual State Production

Df Crops and Animal Products

In 80-km Radius Circle

Regional Productivity Factor:

Animal Grazing Factor:
Veg: 2.8E7 kilogram

Milk: 1.1 E8 liter

Meat: $7.2 \mathrm{E} 7 \mathrm{ki} \log r a \mathrm{~m}$

0.4

1

Meteorology Period of Record: 1 JAN 73 - 31 DEC 73 Recovery: $98 \%$

Fish:

Invertebrates:
Average Dilution Flow

from Plant: $6,400 \mathrm{ft}^{3} / \mathrm{s}$

Edible Harvest: (a)

Dilution Factor: 0.001

Edible Harvest: (a)

Dilution Factor: 0.002

(a) No catch data given in DES (1972), so generic consumption rates used (Table A-1). 
POPULATION DOSE-COMMITMENT ESTIMATES AND

AVERAGE INDIVIDUAL DOSE-COMMITMENT HISTOGRAM FOR

TURKEY POINT 3 AND 4

Dose Commitments (person-rem) from Waterborne Pathways

Iotal Body GI-LII $\underline{\text { Thyroid }}$ Bone Liver

$\begin{array}{llllll}\text { Infant } & 0.0 \mathrm{E}+00 & 0.0 \mathrm{E}+00 & 0.0 \mathrm{E}+00 & 0.0 \mathrm{E}+00 & 0.0 \mathrm{E}+00 \\ \text { Child } & 1.4 \mathrm{E}-03 & 2.9 \mathrm{E}-03 & 1.4 \mathrm{E}-04 & 5.4 \mathrm{E}-03 & 3.6 \mathrm{E}-03 \\ \text { Teen } & 9.2 \mathrm{E}-04 & 5.7 \mathrm{E}-03 & 1.1 \mathrm{E}-04 & 3.0 \mathrm{E}-03 & 2.8 \mathrm{E}-03 \\ \text { Adult } & 5.8 \mathrm{E}-03 & 4.9 \mathrm{E}-02 & 7.4 \mathrm{E}-04 & 1.8 \mathrm{E}-02 & 1.6 \mathrm{E}-02 \\ \text { TOTAL } & 8.1 \mathrm{E}-03 & 5.7 \mathrm{E}-02 & 9.9 \mathrm{E}-04 & 2.6 \mathrm{E}-02 & 2.2 \mathrm{E}-02\end{array}$

Dose Commitments (person-rem) from Airborne Pathways

Tota] Body GI-LII Thyroid Bone Liver

$\begin{array}{lllllll}\text { Infant } & 1.4 \mathrm{E}-03 & 1.4 \mathrm{E}-03 & 8.7 \mathrm{E}-03 & 7.7 \mathrm{E}-04 & 1.4 \mathrm{E}-03 & 1.5 \mathrm{E}-03 \\ \text { Child } & 2.0 \mathrm{E}-02 & 2.0 \mathrm{E}-02 & 6.1 \mathrm{E}-02 & 8.5 \mathrm{E}-03 & 2.0 \mathrm{E}-02 & 2.1 \mathrm{E}-02 \\ \text { Teen } & 1.5 \mathrm{E}-02 & 1.5 \mathrm{E}-02 & 3.1 \mathrm{E}-02 & 6.1 \mathrm{E}-03 & 1.5 \mathrm{E}-02 & 1.6 \mathrm{E}-02 \\ \text { Adult } & 9.0 \mathrm{E}-02 & 9.0 \mathrm{E}-02 & 1.5 \mathrm{E}-01 & 3.7 \mathrm{E}-02 & 9.0 \mathrm{E}-02 & 9.4 \mathrm{E}-02 \\ \text { TOTAL } & 1.3 \mathrm{E}-01 & 1.3 \mathrm{E}-01 & 2.5 \mathrm{E}-01 & 5.2 \mathrm{E}-02 & 1.3 \mathrm{E}-01 & 1.3 \mathrm{E}-01\end{array}$

Production/Consumption factors:

Produce: $0.021 \quad$ Milk: 0.12 Meat: 0.13

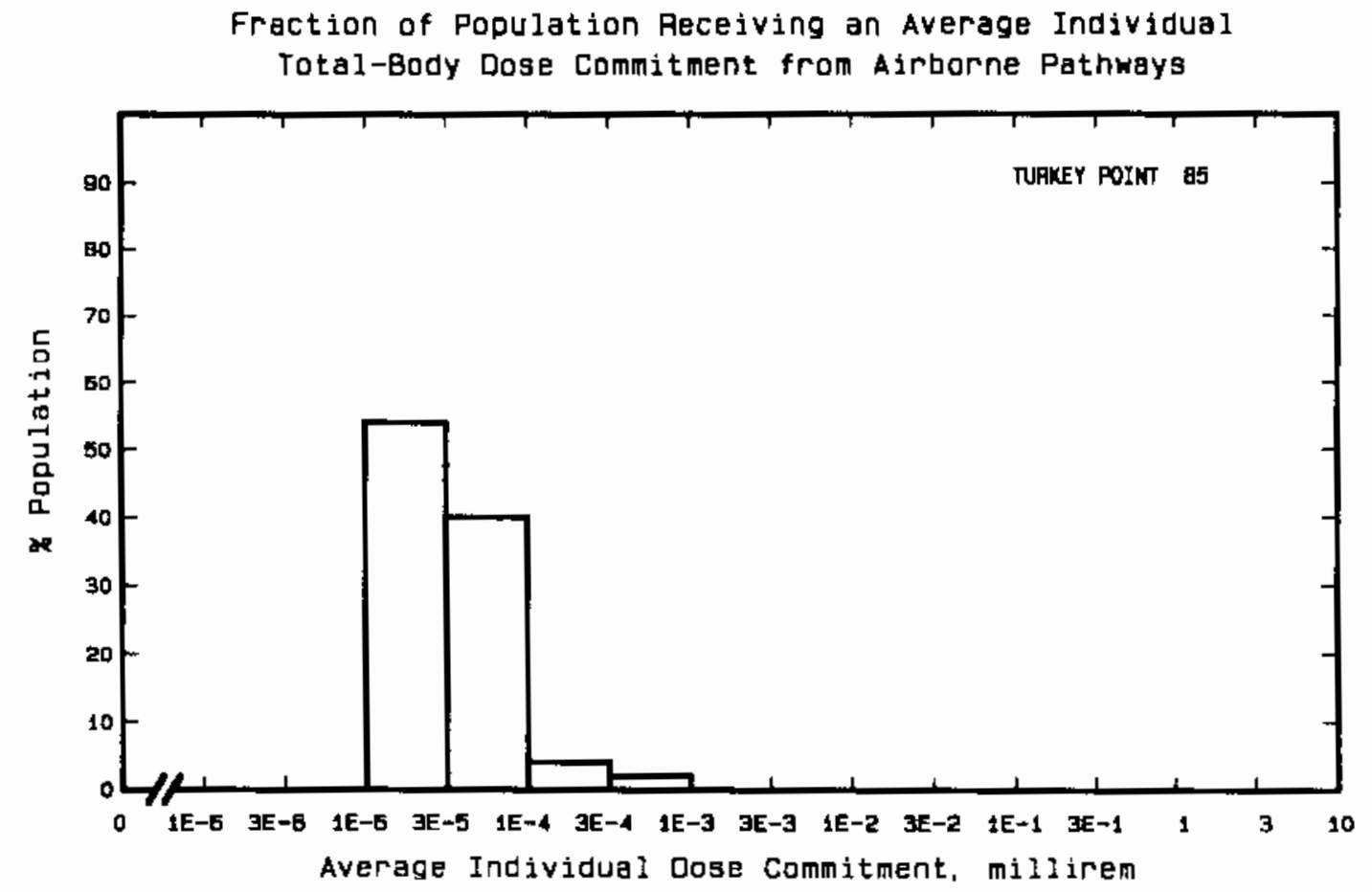


Site: VERMONT YANKEE

VERNON, VERMONT

Location: $\quad$ N $42.7803^{\circ}$ W $72.5158^{\circ}$

POPULATION DATA

Total Population Within 2-to-80-km Region: 1.4E6

Major Metropolitan Centers Within Region:

Center Population Location

Springfield-Chicopee-Holyoke SMSA $\quad 550,000 \quad 70 \mathrm{~km} \quad \mathrm{~S}$

Worcester SMSA (1/2) 200,000 $\quad 80 \mathrm{~km} \quad$ SE

Pittsfield

$95,000 \quad 71 \mathrm{~km} \quad \mathrm{SW}$

Fitchburg

$42,000 \quad 63 \mathrm{~km} \quad \mathrm{ESE}$

Leominster

37,000

$68 \mathrm{~km} \quad \mathrm{ESE}$

Keene

22,000

$26 \mathrm{~km} \quad \mathrm{NW}$

SITE-SPECIFIC DATA - AIRBORNE PATHWAYS

Average Annual State Production

of Crops and Animal Products

In $80-\mathrm{km}$ Radius Circle

Regional Productivity Factor:

Animal Grazing Factor:
Veg: 4.4E6 kilogram

Milk: $7.3 \mathrm{~EB}$ liter

Meat: 2.7E7 kilogram

1

0.4

Meteorology Period of Record: I APR 75 - 31 MAR 76 Recovery: 97\%

SITE-SPECIFIC DATA - WATERBORNE PATHWAYS via CONNECTICUT RIVER at VERNON

POND

Average River $\mathrm{Flow}$

at Site: $10,000 \mathrm{ft}^{3} / \mathrm{s}$

Drinking Water:

Exposed Population: None

Fish:

Edible Harvest: (a)

Dilution Factor: (a)

(a) No radionuclides released in liquid effluents reported (Tichler, et al. 1988). 


\section{POPULATION DOSE-COMNITMENT ESTIMATES AND \\ AVERAGE INDIVIDUAL DOSE-COMMITMENT HISTOGRAM FOR \\ VERMONT YANKEE}

Dose Commitments (person-rem) from Waterborne Pathways

Total Body GI-LII Thyroid Bone Liver

Infant

Child

Teen

(No Waterborne Pathway Doses)

Adult

TOTAL

Dose Commitments (person-rem) from Airborne Pathways

Total Body GI-LII Thyroid Bone Liver

$\begin{array}{lllllll}\text { Infant } & 1.5 \mathrm{E}-03 & 1.5 \mathrm{E}-03 & 1.7 \mathrm{E}-03 & 1.3 \mathrm{E}-03 & 1.5 \mathrm{E}-03 & 1.7 \mathrm{E}-03 \\ \text { Child } & 1.6 \mathrm{E}-02 & 1.6 \mathrm{E}-02 & 1.8 \mathrm{E}-02 & 1.4 \mathrm{E}-02 & 1.7 \mathrm{E}-02 & 2.0 \mathrm{E}-02 \\ \text { Teen } & 1.2 \mathrm{E}-02 & 1.2 \mathrm{E}-02 & 1.2 \mathrm{E}-02 & 1.0 \mathrm{E}-02 & 1.2 \mathrm{E}-02 & 1.5 \mathrm{E}-02 \\ \text { Adult } & 7.0 \mathrm{E}-02 & 7.0 \mathrm{E}-02 & 7.3 \mathrm{E}-02 & 6.3 \mathrm{E}-02 & 7.0 \mathrm{E}-02 & 8.4 \mathrm{E}-02 \\ \text { TOTAL } & 1.0 \mathrm{E}-01 & 1.0 \mathrm{E}-01 & 1.1 \mathrm{E}-01 & 8.9 \mathrm{E}-02 & 1.0 \mathrm{E}-01 & 1.2 \mathrm{E}-01\end{array}$

Production/Consumption factors:

Produce: $0.016 \quad$ Mi1k: $4.0 \quad$ Meat: 0.24

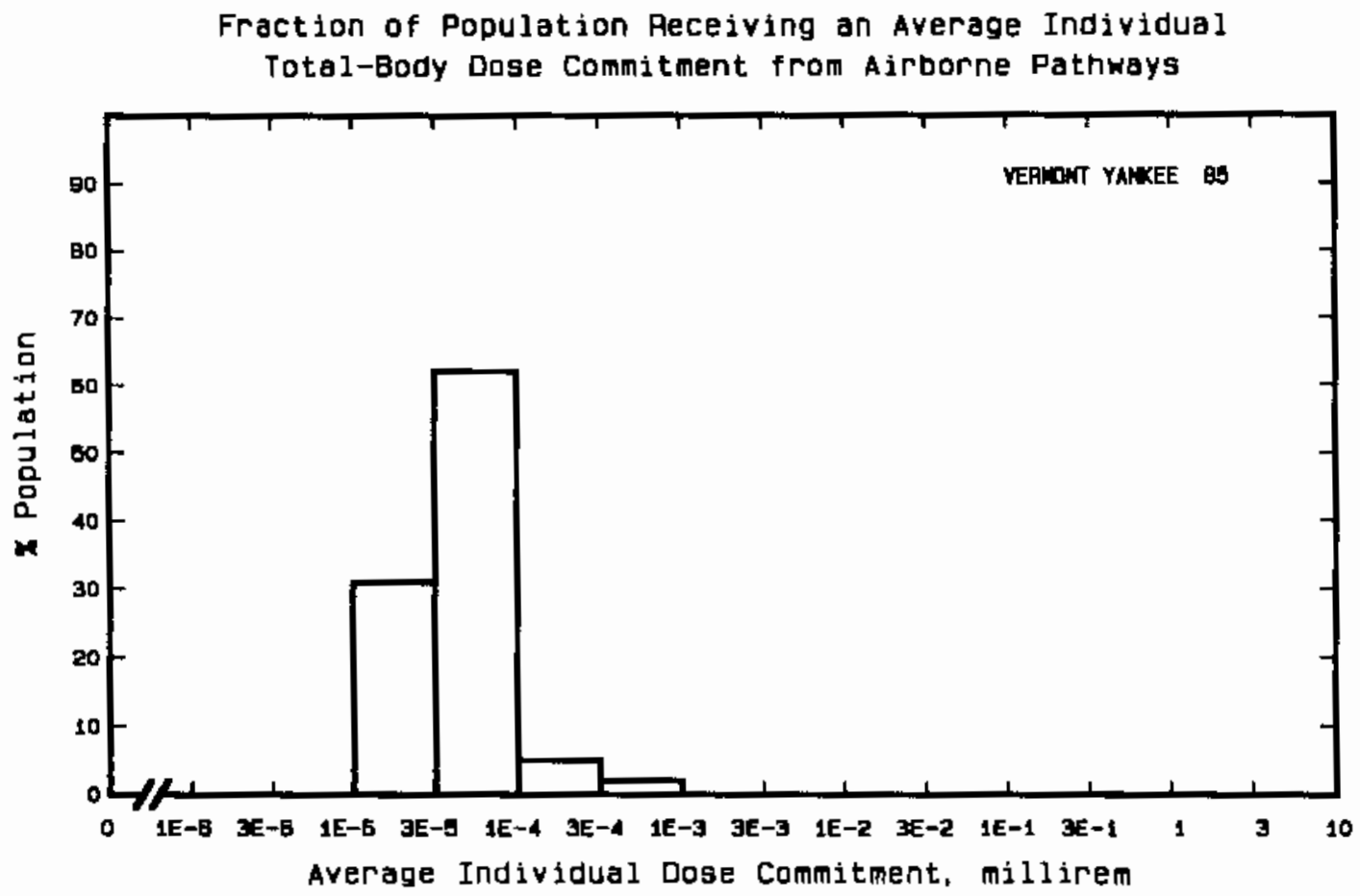


Site: WATERFORD

TAFT, LOUISIANA

Location: N $29.9953^{\circ} \quad$ W $90.4728^{\circ}$

POPULATION DATA

Total Population Within 2-to-80-km Region: 1.9E6

Major Metropolitan Centers Within Region:

$\quad$ Center
New Orleans MSA
Metaire
Kenner
Marrero
Houma

Population
$1,300,000$
140,000
30,000
29,000
31,000

Location

$32 \mathrm{~km} \mathrm{E}$

$26 \mathrm{~km} \quad \mathrm{E}$

$16 \mathrm{~km} \quad \mathrm{E}$

$32 \mathrm{~km}$ ESE

Houma

SITE-SPECIFIC DATA - AIRBORNE PATHWAYS

Average Annual State Production

Veg: $1.8 \mathrm{E} 6$ kilogram

of Crops and Animal Products

Milk: $7.8 \mathrm{E} 7$ liter

In $80-\mathrm{km}$ Radius Circle

Meat: 6. JE7 kilogram

Regional Productivity Factor:

0.6

Animal Grazing Factor:

1.0

Meteorology Period of Record: 2 FEB 77 - 1 FEB 78 Recovery: $89 \%$

SITE-SPECIFIC DATA - WATERBORNE PATHWAYS via MISSISSIPPI RIVER AND GULF

Drinking Water:

Fish:

Gulf Invertebrates:
Average River Flow

at Site: $490,000 \mathrm{ft}^{3} / \mathrm{s}$

Exposed Population: $1,100,000$

Difution Factor: 1

Edible Harvest: $4.5 \mathrm{E} 3 \mathrm{~kg} / \mathrm{yr}$ (River) Dilution Factor: 1

Edible Harvest: $9.1 \mathrm{E5} \mathrm{kg} / \mathrm{yr}$ (Gulf)

Dilution Factor: 0.1

Edible Harvest: $5.1 \mathrm{E} 6 \mathrm{~kg} / \mathrm{yr}$ Dilution Factior: 0.1 
POPULATION DOSE-COMMITMENT ESTIMATES AND AVERAGE INDIVIDUAL DOSE-COMMITMENT HISTOGRAM FOR

\section{WATERFORD}

Dose Commitments (person-rem) from Waterborne Pathways

\begin{tabular}{lccccc} 
& Total Body & GI-LLI & Thyroid & Bone & Liver \\
\cline { 2 - 3 } & & & & & \\
Infant & $6.0 \mathrm{E}-05$ & $7.3 \mathrm{E}-05$ & $1.6 \mathrm{E}-03$ & $1.0 \mathrm{E}-05$ & $6.3 \mathrm{E}-05$ \\
Child & $7.0 \mathrm{E}-05$ & $1.1 \mathrm{E}-03$ & $1.1 \mathrm{E}-02$ & $1.3 \mathrm{E}-04$ & $6.9 \mathrm{E}-04$ \\
Teen & $2.7 \mathrm{E}-05$ & $8.1 \mathrm{E}-04$ & $3.6 \mathrm{E}-03$ & $4.0 \mathrm{E}-05$ & $2.8 \mathrm{E}-04$ \\
Adult & $2.2 \mathrm{E}-03$ & $7.1 \mathrm{E}-03$ & $2.5 \mathrm{E}-02$ & $2.5 \mathrm{E}-04$ & $2.2 \mathrm{E}-03$ \\
TOTAL & $3.2 \mathrm{E}-03$ & $9.0 \mathrm{E}-03$ & $4.1 \mathrm{E}-02$ & $4.2 \mathrm{E}-04$ & $3.2 \mathrm{E}-03$
\end{tabular}

Dose Commitments (person-rem) from Airborne Pathways

Total Body GI-LLI Thyroid Bone Liver Lung

$\begin{array}{lllllll}\text { Infant } & 6.8 \mathrm{E}-03 & 6.8 \mathrm{E}-03 & 8.3 \mathrm{E}-03 & 6.8 \mathrm{E}-03 & 6.8 \mathrm{E}-03 & 7.4 \mathrm{E}-03 \\ \text { Child } & 7.5 \mathrm{E}-02 & 7.5 \mathrm{E}-02 & 8.6 \mathrm{E}-02 & 7.5 \mathrm{E}-02 & 7.5 \mathrm{E}-02 & 8.4 \mathrm{E}-02 \\ \text { Teen } & 5.5 \mathrm{E}-02 & 5.5 \mathrm{E}-02 & 6.0 \mathrm{E}-02 & 5.5 \mathrm{E}-02 & 5.5 \mathrm{E}-02 & 6.6 \mathrm{E}-02 \\ \text { Adult } & 3.3 \mathrm{E}-01 & 3.3 \mathrm{E}-01 & 3.5 \mathrm{E}-01 & 3.3 \mathrm{E}-01 & 3.3 \mathrm{E}-01 & 3.7 \mathrm{E}-01 \\ \text { TOTAL } & 4.7 \mathrm{E}-01 & 4.7 \mathrm{E}-01 & 5.1 \mathrm{E}-01 & 4.7 \mathrm{E}-01 & 4.7 \mathrm{E}-01 & 5.3 \mathrm{E}-01\end{array}$

Production/Consumption factors:

Produce: 0.0029 Milk: $0.19 \quad$ Meat: 0.25

Fraction of Population Receiving an Average Individual Tota1-Body Dose Commitment from Airborne Pathways

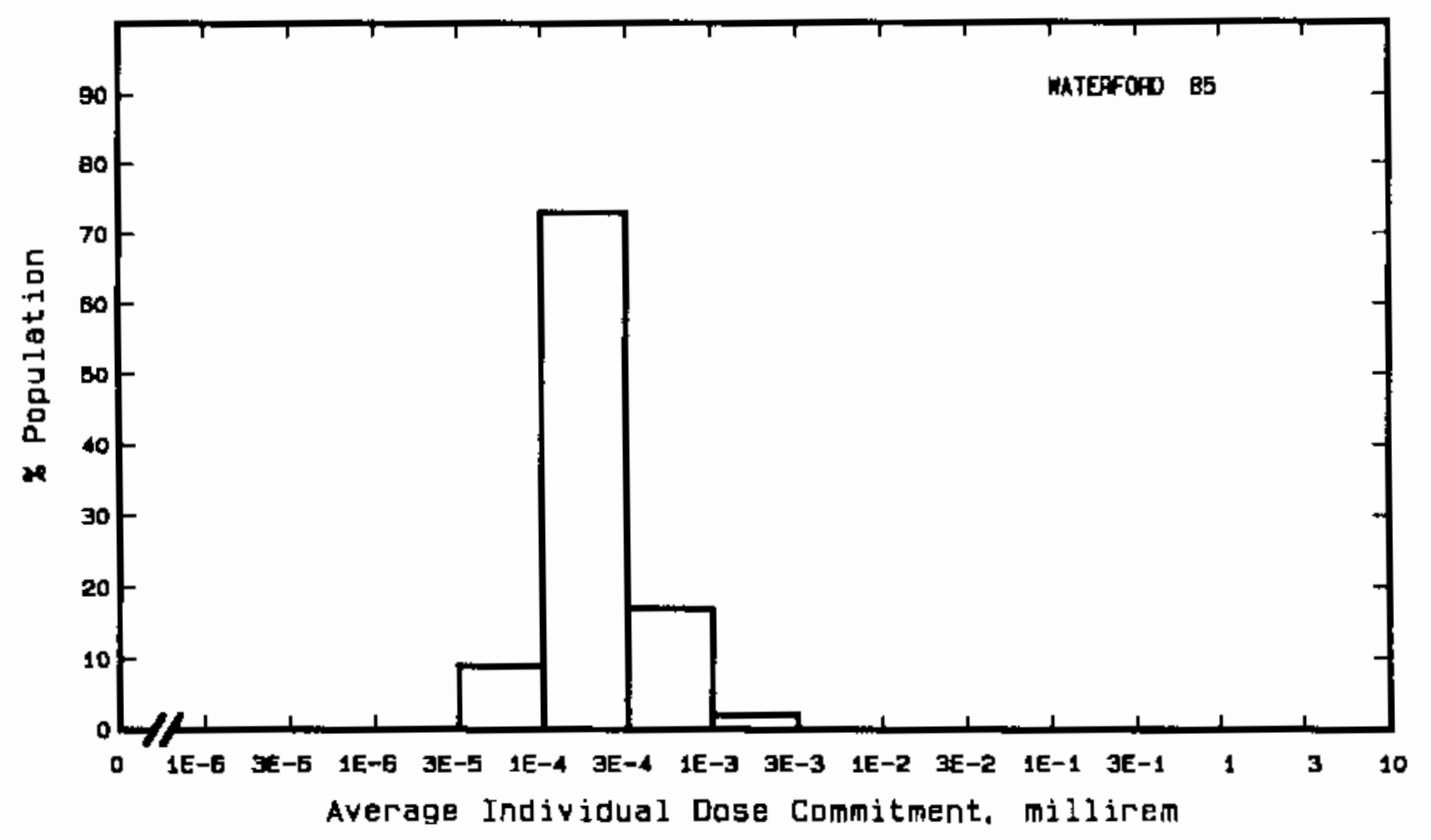


Site: WNP-2

Location: N $46.2833^{\circ}$
RICHLAND, WASHINGTON

W $119.2916^{\circ}$

POPULATION DATA

Total Population Within 2-to-80-km Region: 2.6E5

Major Metropolitan Centers Within Region:

\begin{tabular}{lcccc}
\multicolumn{1}{c}{ Center } & Population & & \multicolumn{2}{c}{ Location } \\
Richland & & & & \\
Kennewick & 34,000 & & $14 \mathrm{~km}$ & $\mathrm{~S}$ \\
Pasco & 35,000 & & $26 \mathrm{~km}$ & SSE \\
Moses Lake & 19,000 & & $28 \mathrm{~km}$ & SSE \\
Mose & 11,000 & & $73 \mathrm{~km}$ & $\mathrm{~N}$
\end{tabular}

SITE-SPECIFIC DATA - AIRBORNE PATHWAYS

Average Annual State Production

of Crops and Animal Products

In $80-\mathrm{km}$ Radius Circle

Regional Productivity Factor:

Animal Grazing Factor:
Veg: 2.1E8 kilogram

Milk: 1.2E8 liter

Meat: $3.2 E 7$ kilogram

0.8

0.7

Meteorology Period of Record: 1 APR $75-31$ MAR 76 Recovery: 93\%

SITE-SPECIFIC DATA - WATERBORNE PATHWAYS via COIUMBIA RIVER

Average River Flow at Site: $115,000 \mathrm{ft}^{3} / \mathrm{s}$

Drinking Water:

Fish:
Exposed Poputation: 71,000 Dilution Factior: $3.4(a)$

Edible Harvest: $7.5 \mathrm{E} 3 \mathrm{~kg} / \mathrm{yr}$

Dilution Factor: 1

(a) Effective factor for the cities of Richland, Kennewick, and Pasco over complete river dilution. 


\section{POPULATION DOSE-COMMITMENT ESTIMATES AND \\ AVERAGE INDIVIDUAL DOSE-COMMITMENT HISTOGRAM FOR}

WNP-2

Dose Commitments (person-rem) from Waterborne Pathways

Total Body GI-LII $\underline{\text { Thyroid }}$ Bone

$\begin{array}{llllll}\text { Infant } & 7.8 \mathrm{E}-06 & 4.2 \mathrm{E}-06 & 7.8 \mathrm{E}-06 & 2.1 \mathrm{E}-05 & 3.6 \mathrm{E}-06 \\ \text { Child } & 1.1 \mathrm{E}-04 & 5.3 \mathrm{E}-05 & 6.6 \mathrm{E}-05 & 3.1 \mathrm{E}-04 & 3.9 \mathrm{E}-05 \\ \text { Teen } & 3.9 \mathrm{E}-05 & 2.9 \mathrm{E}-05 & 2.2 \mathrm{E}-05 & 1.1 \mathrm{E}-04 & 1.5 \mathrm{E}-05 \\ \text { Adult } & 3.2 \mathrm{E}-04 & 2.4 \mathrm{E}-04 & 1.7 \mathrm{E}-04 & 8.6 \mathrm{E}-04 & 1.2 \mathrm{E}-04 \\ \text { TOTAL } & 4.7 \mathrm{E}-04 & 3.3 \mathrm{E}-04 & 2.7 \mathrm{E}-04 & 1.3 \mathrm{E}-03 & 1.8 \mathrm{E}-04\end{array}$

Dose Commitments (person-rem) from Airborne Pathways

Total Body GI-LII Thyroid Bone Liver Lung

$\begin{array}{lllllll}\text { Infant } & 2.2 \mathrm{E}-04 & 3.3 \mathrm{E}-04 & 5.4 \mathrm{E}-04 & 3.6 \mathrm{E}-04 & 4.9 \mathrm{E}-04 & 4.5 \mathrm{E}-04 \\ \text { Child } & 3.3 \mathrm{E}-03 & 4.4 \mathrm{E}-03 & 5.4 \mathrm{E}-03 & 5.9 \mathrm{E}-03 & 6.1 \mathrm{E}-03 & 6.2 \mathrm{E}-03 \\ \text { Teen } & 2.1 \mathrm{E}-03 & 3.9 \mathrm{E}-03 & 2.8 \mathrm{E}-03 & 2.8 \mathrm{E}-03 & 3.4 \mathrm{E}-03 & 5.0 \mathrm{E}-03 \\ \text { Adult } & 1.1 \mathrm{E}-02 & 2.0 \mathrm{E}-02 & 1.3 \mathrm{E}-02 & 1.3 \mathrm{E}-02 & 1.5 \mathrm{E}-02 & 2.1 \mathrm{E}-02 \\ \text { TOTAL } & 1.7 \mathrm{E}-02 & 2.9 \mathrm{E}-02 & 2.2 \mathrm{E}-02 & 2.2 \mathrm{E}-02 & 2.5 \mathrm{E}-02 & 3.3 \mathrm{E}-02\end{array}$

Production/Consumption factors:

Produce: $3.9 \quad$ Milk: $2.8 \quad$ Meat: 1.2

Fraction of Population Receiving an Average Individual Total-Body Dose Commitment from Airborne Pathways

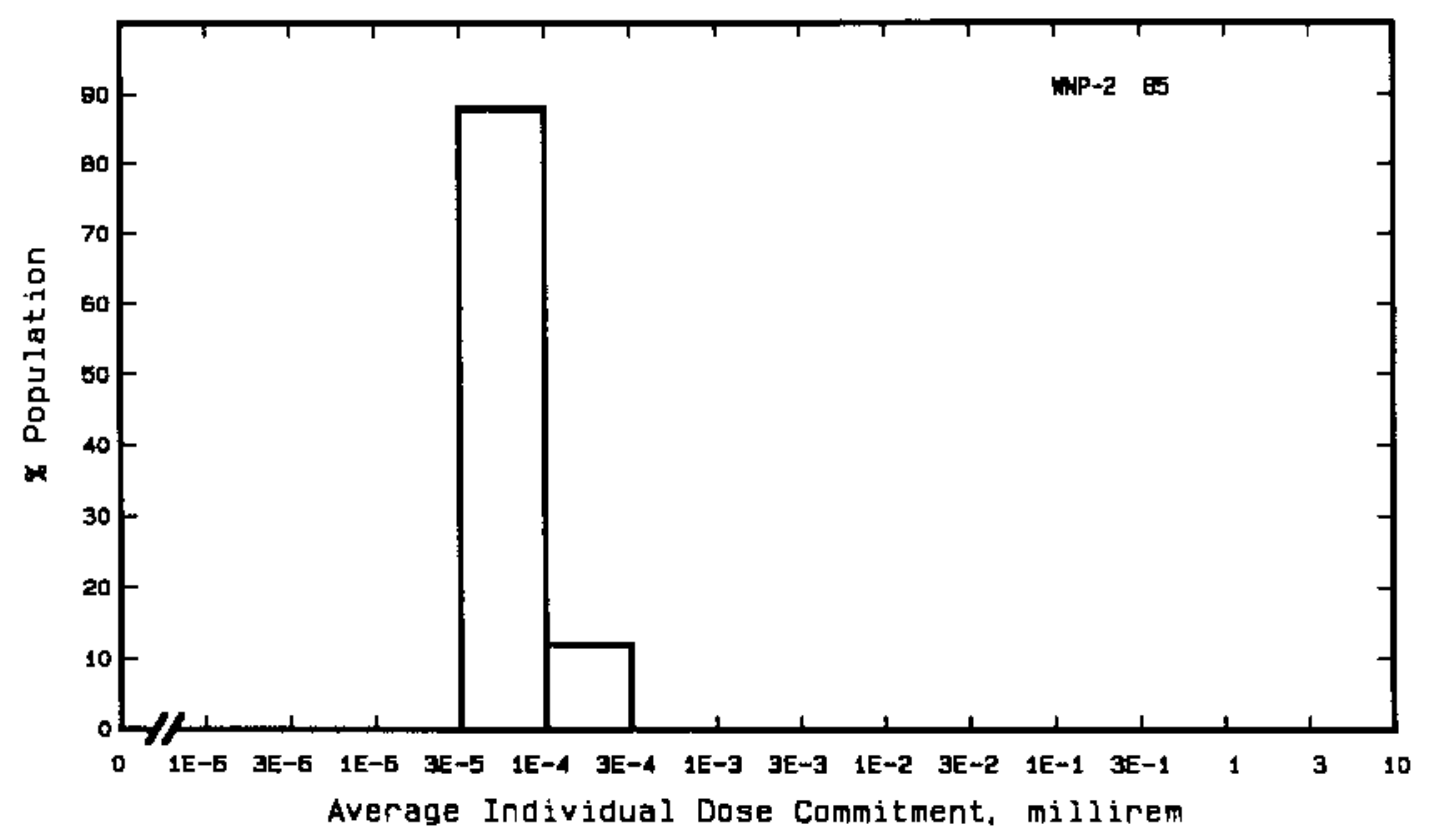


Site: WOLF CREEK

BURLINGTON, KANSAS

Location: $\quad$ N $39.0267^{\circ} \quad$ W $84.7233^{\circ}$

POPULATION DATA

Total Population Within 2-to-80-km Region: $1.8 E 5$

Major Metropolitan Centers Within Region:

Center

Chanute

Emporia

ottawa
Population

11,000

25,000

11,000
Location

$62 \mathrm{~km} \quad \mathrm{SSE}$

$42 \mathrm{~km} \quad$ WNW

$58 \mathrm{~km} \quad \mathrm{NW}$

SITE-SPECIFIC DATA - AIRBORNE PATHWAYS

Average Annual State Production

of Crops and Animal Products

In $80-\mathrm{km}$ Radius Circle

Regional Productivity Factor:

Animal Grazing Factor:

Meteorology Period of Record: 3 years
Veg: $2.4 \mathrm{E} 8 \mathrm{kilogram}$

Milk: 6.5E7 liter

Meat: $1.6 \mathrm{E} 8$ kilogram

0.95

0.5

SITE-SPECIFIC DATA - WATERBORNE PATHWAYS via NEOSHO RIVER

Average River Flow

at Site: $1,300 \mathrm{ft}^{3} / \mathrm{s}$

Drinking Water:

Exposed Population: None

Dilution Factor:

Fish:

Edible Harvest: $1.8 \mathrm{~kg} / \mathrm{yr}$ (a)

Dilution Factor: 1

(a) Average consumption rate per person from FES. 
POPULATION DOSE-COMMITMENT ESTIMATES AND AVERAGE INDIVIDUAL DOSE-COMMITMENT HISTOGRAM FOR

WOLF CREEK

Dose Commitments (person-rem) from Waterborne Pathways

\begin{tabular}{|c|c|c|c|c|c|}
\hline & Iotal Body & $\underline{G I-L L I}$ & Thyroid & Bone & Liver \\
\hline Infan & $0.0 E+00$ & $0.0 E+00$ & $0.0 E+00$ & $0.0 \mathrm{E}+00$ & $0.0 E+00$ \\
\hline Child & $5.1 \mathrm{E}-03$ & $8.2 E-02$ & $3.1 \mathrm{E}-03$ & $9.9 \mathrm{E}-03$ & $1.7 \mathrm{E}-02$ \\
\hline Teen & $7.1 \mathrm{E}-03$ & $1.7 \mathrm{E}-01$ & $2.3 \mathrm{E}-03$ & $6.2 E-03$ & $1.5 \mathrm{E}-02$ \\
\hline Adult & $6.9 \mathrm{E}-02$ & $1.5 \mathrm{E}+00$ & $1.6 \mathrm{E}-02$ & $3.7 E-02$ & $9.0 \mathrm{E}-02$ \\
\hline TOTAL & $8.1 \mathrm{E}-02$ & $1.7 \mathrm{E}+00$ & 2.1E-02 & $5.3 \mathrm{E}-02$ & $1.2 \mathrm{E}-0 \mathrm{l}$ \\
\hline
\end{tabular}

Dose Commitments (person-rem) from Airborne Pathways

Total Body GI-LLI Thyroid Bone Liver Lung

$\begin{array}{lllllll}\text { Infant } & 4.1 \mathrm{E}-04 & 4.1 \mathrm{E}-04 & 4.1 \mathrm{E}-04 & 3.9 \mathrm{E}-04 & 4.1 \mathrm{E}-04 & 4.2 \mathrm{E}-04 \\ \text { Child } & 4.8 \mathrm{E}-03 & 4.8 \mathrm{E}-03 & 4.9 \mathrm{E}-03 & 4.3 \mathrm{E}-03 & 4.8 \mathrm{E}-03 & 4.9 \mathrm{E}-03 \\ \text { Teen } & 3.4 \mathrm{E}-03 & 3.4 \mathrm{E}-03 & 3.5 \mathrm{E}-03 & 3.1 \mathrm{E}-03 & 3.4 \mathrm{E}-03 & 3.6 \mathrm{E}-03 \\ \text { Adult } & 2.1 \mathrm{E}-02 & 2.1 \mathrm{E}-02 & 2.1 \mathrm{E}-02 & 1.9 \mathrm{E}-02 & 2.1 \mathrm{E}-02 & 2.1 \mathrm{E}-02 \\ \text { TOTAL } & 2.9 \mathrm{E}-02 & 2.9 \mathrm{E}-02 & 2.9 \mathrm{E}-02 & 2.7 \mathrm{E}-03 & 2.9 \mathrm{E}-02 & 3.0 \mathrm{E}-02\end{array}$

Production/Consumption factors:
Produce: 6.5
Mi1k: 2.6
Meat: 11

Fraction of Population Receiving an Average Individual

Tota1-Body Dose Commitment from Airborne Pathways

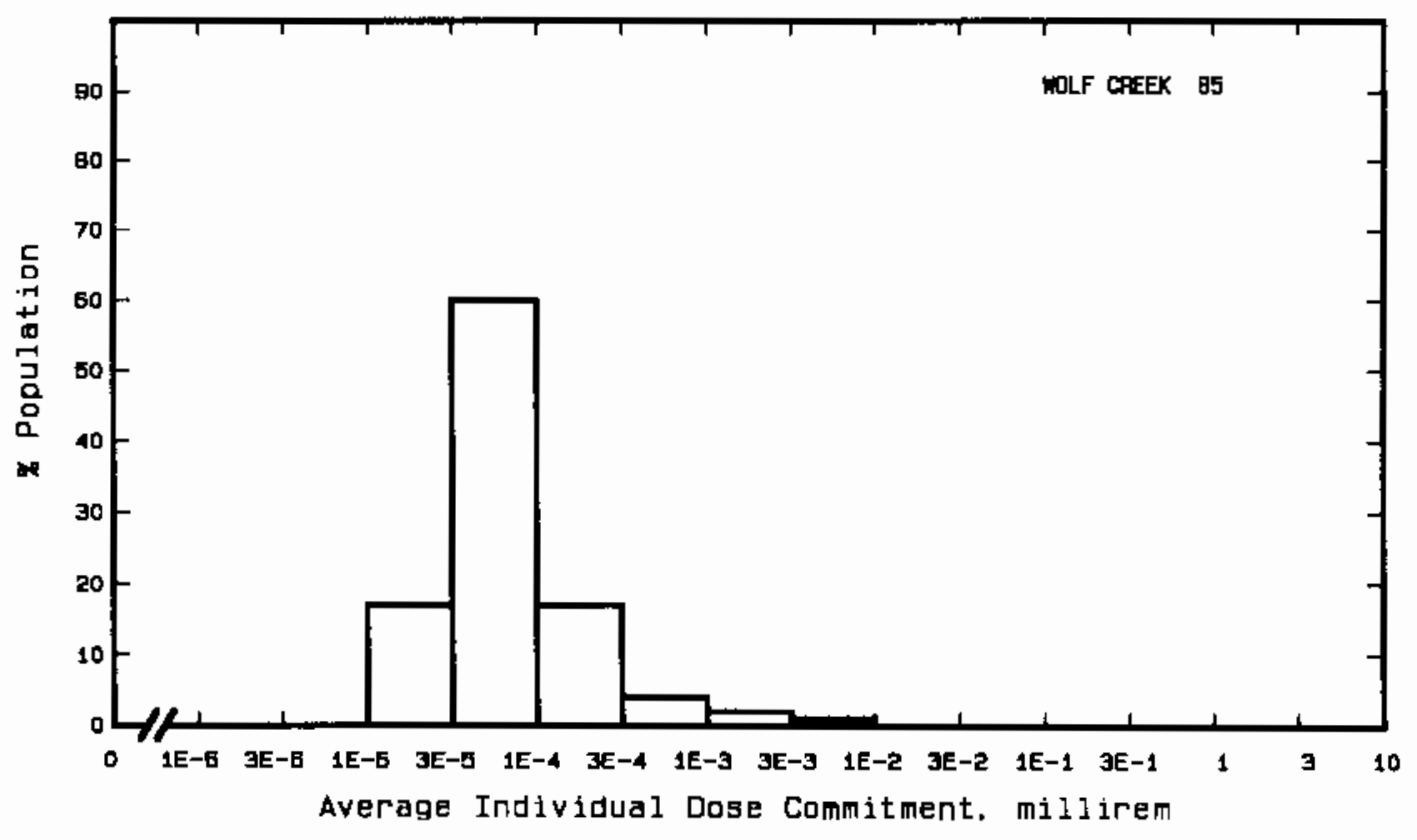


Site: YANKEE ROWE

Location: $\quad \mathrm{N} \quad 42.7281^{\circ}$
ROWE, MASSACHUSETTS

W $72.9289^{\circ}$

POPULATION DATA

Total Population Within 2-to-80-km Region: 1.6E6

Major Metropolitan Centers Withir Region:

Center

Springfield-Chicopee-Holyoke SMSA

Albany

Pittsfield SMSA

Troy

Anherst
Population!

530,000

100,000

91,000

57,000

18,000
Location

$74 \mathrm{~km} \quad$ SSE

$68 \mathrm{~km} \quad W$

$41 \mathrm{~km} \quad \mathrm{SW}$

$62 \mathrm{~km} \quad \mathrm{~W}$

$51 \mathrm{~km} \quad \mathrm{SE}$

SITE-SPECIFIC DATA - AIRBORNE PATHWAYS

Average Annual State Production

of Crops and Animal Products

In $80-\mathrm{km}$ Radius Circle

Regional Productivity Factor:

Animal Grazing Factor:
Veg: $2.0 E 7$ kilogram

Milk: 2.6E8 liter

Meat: $1.6 \mathrm{E} 7 \mathrm{ki}$ logram

1

0.5

Meteorology Period of Record: 1 OCT 71 - 30 SEP 72 Recovery: $94 \%$

SITE-SPECIFIC DATA - WATERBORNE PATHWAYS via DEERFIELD RIVER

Average River $\mathrm{Flow}$

at Site: $570 \mathrm{ft}^{3} / \mathrm{s}$

Drinking Water:

Exposed Population: None

Fish:

Edible Harvest: (a)

Dilution Factor: $0.025^{(b)}$

(a) No catch data available, so generic consumption rates used (Table A-1).

(b) Ten percent of population obtain $25 \%$ of their fish from river. 


\section{POPULATION DOSE-COMMITMENT ESTIMATES AND}

AVERAGE INDIVIDUAL DOSE-COMMITMENT HISTOGRAM FOR

YANKEE ROWE

Dose Commitments (person-rem) from Waterborne Pathways

Total Body GI-LII Ihyroid Bone Liver

$\begin{array}{llllll}\text { Infant } & 0.0 \mathrm{E}+00 & 0.0 \mathrm{E}+00 & 0.0 \mathrm{E}+00 & 0.0 \mathrm{E}+00 & 0.0 \mathrm{E}+00 \\ \text { Child } & 1.5 \mathrm{E}-02 & 5.1 \mathrm{E}-03 & 7.5 \mathrm{E}-03 & 6.4 \mathrm{E}-02 & 5.7 \mathrm{E}-02 \\ \text { Teen } & 2.2 \mathrm{E}-02 & 4.6 \mathrm{E}-03 & 5.2 \mathrm{E}-03 & 3.9 \mathrm{E}-02 & 4.8 \mathrm{E}-02 \\ \text { Adult } & 2.2 \mathrm{E}-01 & 3.3 \mathrm{E}-02 & 3.4 \mathrm{E}-02 & 2.3 \mathrm{E}-01 & 2.9 \mathrm{E}-01 \\ \text { TDTAL } & 2.5 \mathrm{E}-01 & 4.3 \mathrm{E}-02 & 4.7 \mathrm{E}-02 & 3.3 \mathrm{E}-01 & 3.9 \mathrm{E}-01\end{array}$

Dose Commitments (person-rem) from Airborne Pathways

Total Body GI-LII Thyroid Bone Liver Lung

$\begin{array}{lllllll}\text { Infant } & 5.0 \mathrm{E}-03 & 5.0 \mathrm{E}-03 & 5.6 \mathrm{E}-03 & 1.9 \mathrm{E}-02 & 5.0 \mathrm{E}-03 & 5.1 \mathrm{E}-03 \\ \text { Child } & 3.8 \mathrm{E}-02 & 3.8 \mathrm{E}-02 & 4.1 \mathrm{E}-02 & 1.4 \mathrm{E}-01 & 3.8 \mathrm{E}-02 & 3.9 \mathrm{E}-02 \\ \text { Teen } & 1.7 \mathrm{E}-02 & 1.7 \mathrm{E}-02 & 1.9 \mathrm{E}-02 & 4.6 \mathrm{E}-02 & 1.7 \mathrm{E}-02 & 1.8 \mathrm{E}-02 \\ \text { Adult } & 8.0 \mathrm{E}-02 & 8.0 \mathrm{E}-02 & 8.5 \mathrm{E}-02 & 1.6 \mathrm{E}-01 & 8.0 \mathrm{E}-02 & 8.4 \mathrm{E}-02 \\ \text { TOTAL } & 1.4 \mathrm{E}-01 & 1.4 \mathrm{E}-01 & 1.5 \mathrm{E}-01 & 3.6 \mathrm{E}-01 & 1.4 \mathrm{E}-01 & 1.5 \mathrm{E}-01\end{array}$

Production/Consumption factors:

Produce: 0.063 Milk: 1.2 Meat: 0.13

Fraction of Population Receiving an Average Individual

Total-Body Dose Commitment from Airborne Pathways

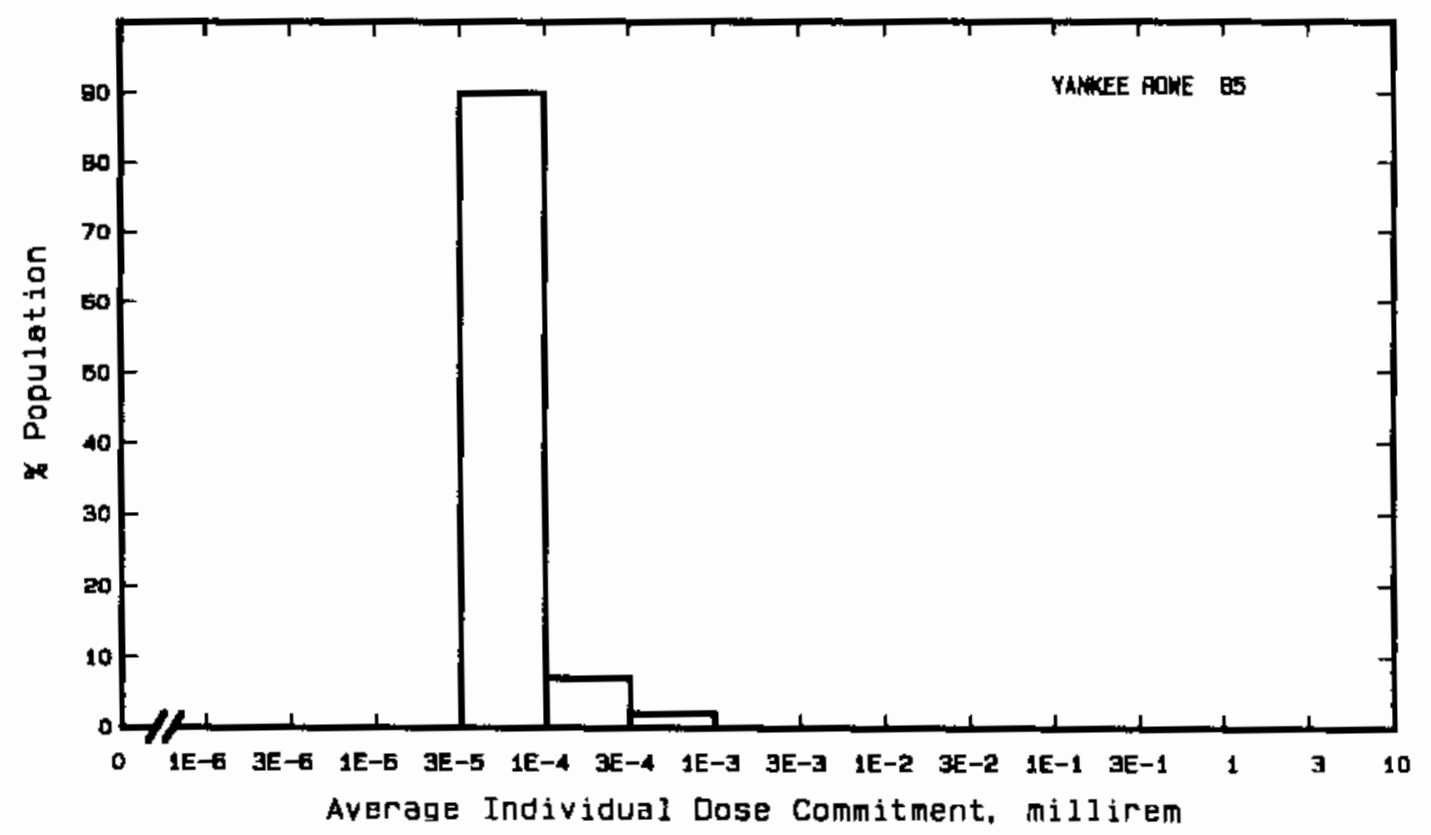


Site: ZION

ZION, ILLINOIS

Location: $\quad$ N $42.4456^{\circ} \quad$ W $87.8022^{\circ}$

POPULATION DATA

Total Population Within 2-to-80-km Region: 7.2E6

Major Metropolitan Centers Within Region:

\begin{tabular}{lrrrr}
\multicolumn{1}{c}{ Center } & Population & \multicolumn{2}{c}{ Location } \\
Chicago SMSA & (2/3) & $4,700,000$ & $66 \mathrm{~km}$ & $\mathrm{~S}$ \\
Milwaukee SMSA & $1,400,000$ & $65 \mathrm{~km}$ & $\mathrm{~N}$ \\
Racine SMSA & 170,000 & $30 \mathrm{~km}$ & $\mathrm{~N}$ \\
Kenosha SMSA & 120,000 & $14 \mathrm{~km}$ & $\mathrm{~N}$ \\
Waukesha & 50,000 & $71 \mathrm{~km}$ & NNW
\end{tabular}

SITE-SPECIFIC DATA - AIRBORNE PATHWAYS

Average Annual State Production

of Crops and Animal Products

In $80-\mathrm{km}$ Radius Circle

Regional Productivity Factor:

Animal Grazing Factor:
Veg: $1.1 \mathrm{E} 8 \mathrm{kilogram}$

Milk: 1.8E8 liter

Meat: $1.9 \mathrm{E} 8 \mathrm{kilogram}$

0.5

0.5

Meteorology Period of Record: 1 JAN 74 - 31 DEC 75 Recovery: 88\%

SITE-SPECIFIC DATA - WATERBORNE PATHWAYS via LAKE MICHIGAN

Average Dilution $\mathrm{Flow}$

from Plant: 2,500 $\mathrm{ft}^{3} / \mathrm{s}$

Drinking Water:

Exposed Population: 6,800,000

Dilution Factor: $0.037(\mathrm{a})$

Fish:

Edible Harvest: $5.0 \mathrm{E} 6 \mathrm{~kg} / \mathrm{yr}$ Dilution Factor: $0.01(6)$

(a) Drinking water dilution factor estimated by averaging dilution factors derived from FES (1972) suitably weighted for population.

(b) Dilution factor derived from FES. 


\section{POPULATION DOSE-COMMITMENT ESTIMATES AND AVERAGE INDIVIDUAL DOSE-COMMITMENT HISTOGRAM FOR}

\section{ZION 1 AND 2}

Dose Commitments (person-rem) from Waterborne Pathways

Total Body

GI-LII

Thyroid

Bone

Liver

Infant

$7.1 \mathrm{E}-02$

6. $6 \mathrm{E}-02$

2.6E-01

6. $1 \mathrm{E}-02$

$1.4 \mathrm{E}-01$

Child

9. 3E-01

8. 3E-01

2. $1 E+00$

1. $0 \mathrm{E}+00$

1. $9 E+00$

Teen

5. $4 \mathrm{E}-01$

4. $2 \mathrm{E}-0 \mathrm{l}$

6. 8E-01

3. $8 \mathrm{E}-01$

9. $1 \mathrm{E}-01$

Adult

$5.2 E+00$

$3.8 \mathrm{E}+00$

5. $2 \mathrm{E}+00$

2. $3 \mathrm{E}+00$

$6.2 \mathrm{E}+00$

TDTAL

$6.8 \mathrm{E}+00$

5. $1 \mathrm{E}+00$

8. $2 \mathrm{E}+00$

$3.8 \mathrm{E}+00$

9. $1 \mathrm{E}+00$

Dose Commitments (person-rem) from Airborne Pathways

\section{Total Body GI-LII Ihyroid Bone Liver Lung}

$\begin{array}{lllllll}\text { Infant } & 5.5 \mathrm{E}-03 & 5.5 \mathrm{E}-03 & 6.3 \mathrm{E}-03 & 5.3 \mathrm{E}-03 & 5.5 \mathrm{E}-03 & 5.8 \mathrm{E}-03 \\ \text { Child } & 6.2 \mathrm{E}-02 & 6.2 \mathrm{E}-02 & 6.9 \mathrm{E}-02 & 6.0 \mathrm{E}-02 & 6.2 \mathrm{E}-02 & 6.7 \mathrm{E}-02 \\ \text { Teen } & 4.5 \mathrm{E}-02 & 4.5 \mathrm{E}-02 & 4.9 \mathrm{E}-02 & 4.3 \mathrm{E}-02 & 4.5 \mathrm{E}-02 & 5.2 \mathrm{E}-02 \\ \text { Adult } & 2.7 \mathrm{E}-01 & 2.7 \mathrm{E}-01 & 2.9 \mathrm{E}-01 & 2.6 \mathrm{E}-01 & 2.7 \mathrm{E}-01 & 3.0 \mathrm{E}-01 \\ \text { TOTAL } & 3.9 \mathrm{E}-01 & 3.9 \mathrm{E}-01 & 4.1 \mathrm{E}-01 & 3.7 \mathrm{E}-01 & 3.9 \mathrm{E}-01 & 4.2 \mathrm{E}-01\end{array}$

Production/Consumption factors:

Produce: $0.040 \quad$ Milk: 0.094 Meat: 0.16
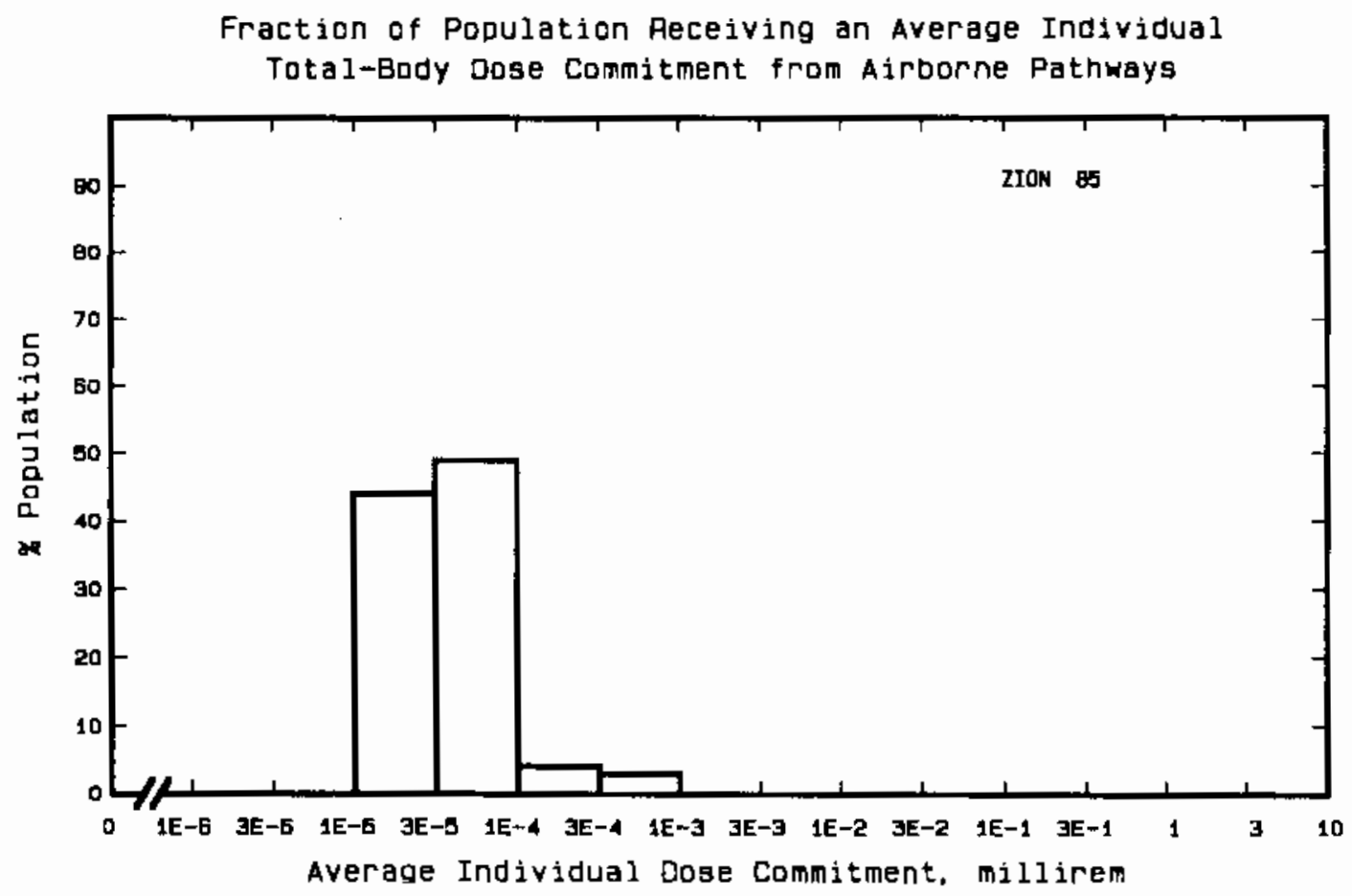



\section{REFERENCES}

Final Environmental Statement Concerning Proposed Rule-Making Action: Numerical Guides for Design Objectives and Limiting Conditions for operatio to Meet the Criterion "As Low As Practicable" for Radioactive Material in Light-Water-Cooled Nuclear Power Reactor Effluents. 1973. WASH-1258, Vol. 1, Directorate of Regulatory Standards, U.S. Atomic Energy Commission, Washington, D.C.

Tichler, J., K. Norden, and J. Congemi. 1988. Radioactive Materials Released from Nuclear Power Plants, Annual Report 1985. NUREG/CR-2907, BNL-NUREG-51581, Vol, 6, U.S. Nuclear Regulatory Commission, Washington, D.C. 



\section{APPENDIX}

\section{MODELS AND GENERIC PARAMETERS}

The calculational models used were primarily those given in the Nuclear Regulatory Commission's Regulatory Guide 1.109 (NRC 1977). Computer programs were written to use these models to generate population dose commitments for four age groups. The percentages of the population comprising the four age groups were $1.44 \%$, infant; $16.0 \%$, child; $11.7 \%$, teenager; and $70.9 \%$, adult (Population Estimates and Projections 1975). Where possible, the site-dependent parameters were taken from the environmental statements issued for each reactor (Table 3 ). The generic parameters used for this study such as consumption rates, occupancy factors and holdup times are given in Tables A-1 and A-2 below. It should be noted that generic consumption rates for aquatic foods and inhalation rates are taken from Regulatory Guide 1.109 (NRC 1977). Bioaccumulation factors and terrestrial food transfer factors were taken from Regulatory Guide 1.109 (NRC 1977). Dose commitment factors for the four age groups were taken from Hoenes and Soldat (1977).

IABLE A-1. Generic Consumption Rates and Occupancy Factors Used for the Study of Average Members of the Population(a)

\begin{tabular}{|c|c|c|c|c|}
\hline Pathway & Infant & Child & Teen-ager & Adult \\
\hline $\begin{array}{l}\text { Fruits, vegetables and grain } \\
(\mathrm{kg} / \mathrm{yr})\end{array}$ & 0 & 200 & 240 & 190 \\
\hline Milk $(L / y r)$ & 170 & 170 & 200 & 110 \\
\hline Meat and poultry $(\mathrm{kg} / \mathrm{yr})$ & 0 & 37 & 59 & 95 \\
\hline Fish $(\mathrm{kg} / \mathrm{yr})^{(\mathrm{b})}$ & 0 & 2.2 & 5.2 & 6.9 \\
\hline Invertebrates $(\mathrm{kg} / \mathrm{yr})$ & 0 & 0.33 & 0.75 & 1.0 \\
\hline Drinking water $(\mathrm{L} / \mathrm{yr})$ & $170^{(c)}$ & 260 & 260 & 370 \\
\hline Inhalation $\left(\mathrm{m}^{3} / \mathrm{yr}\right)$ & $1400^{(d)}$ & 3700 & 8000 & 8000 \\
\hline $\begin{array}{l}\text { Air submersion and ground } \\
\text { irradiation occupancy } \\
\text { factor }\end{array}$ & 0.5 & 0.5 & 0.5 & 0.5 \\
\hline
\end{tabular}

(a) Regulatory Guide 1.109 (NRC 1977).

(b) Both fresh and salt water.

(c) Assumed to be equal to milk consumption.

(d) Same as for maximum individual. 
TABLE A-2. Holdup Times Betweer Harvest and Consumption of Foods (a)

Food

Fruits, grains and vegetables

$\operatorname{Milk}(\mathrm{b})$

Meat ${ }^{(b)}$

Aquatic foods (fish and invertebrates)

Drinking Water
Holdup Time (days)

14

4

20

7

1

(a) Regulatory Guide 1.109 (NRC 1977)

(b) Value given is time after milking or slaughter. For the portion of the time animals were fed stored feed, an additional 90 days was added to the holdup time.

\section{SOURCE TERMS}

The doses were estimated using the measured releases as reported by the site operators for 1985 (Tichler, et al. 1988). (a) These releases include all radionuclides specified by the NRC to be measured and reported by the operators of all commercial nuclear power plants. Radionuclides given as a combination of parent-daughter isotopes such as $\mathrm{Y} / \mathrm{Sr}-90, \mathrm{Zr} / \mathrm{Nb}-95, \mathrm{Ba} / \mathrm{La}-140, \mathrm{I} / \mathrm{Xe}-133$ and $\mathrm{Pr} / \mathrm{Ce}-144$ were divided evenly between the parent and daughter.

The radionuclides used in this study, along with their decay constants, are given in Table A-3. Note that the " $+D$ " after some of the nuclides indicates that the decay energy of the daughter is included with the parent. Thus, whenever a parent nuclide release is specified, the result of the dose calculation is as though an additional equilibrium amount of the daughter nuclide is specified. The daughter nuclide itself is included separately if it can be released independently of the parent and/or if it has a relatively long half-life.

(a) Very shortlived isotopes such as $\mathrm{Kr}-90,9 \%, 93,94, \mathrm{Xe}-139,140,141$, 143 and Rb-88M; those not likely to be produced; and those that were daughters whose decay energies were accourted for in the dose factor for the parent were not included in the dose. 
IABLE A-3. Radionuclides Considered in This Study

\begin{tabular}{|c|c|c|c|c|c|}
\hline №. & Nuclide & $\begin{array}{l}\text { Decay Constant } \\
(1 / \mathrm{sec})\end{array}$ & No. & Nuclide & $\begin{array}{l}\text { Decay Constant } \\
(1 / \mathrm{sec})\end{array}$ \\
\hline 1 & $H-3$ & $1.78 \mathrm{E}-09$ & 43 & $\mathrm{Nb}-97$ & $1.57 \mathrm{E}-04$ \\
\hline 2 & $\mathrm{Be}-10$ & $1.37 \mathrm{E}-14$ & 44 & $\mathrm{M}_{0}-99+\mathrm{D}$ & $2.92 \mathrm{E}-06$ \\
\hline 3 & $C-14$ & $3.83 \mathrm{E}-12$ & 45 & Tc-99M & $3.19 \mathrm{E}-05$ \\
\hline 4 & $\mathrm{~N}-13$ & $1.16 \mathrm{E}-03$ & 46 & $R u-103+D$ & $2.02 \mathrm{E}-07$ \\
\hline 5 & $F-18$ & $1.05 \mathrm{E}-04$ & 47 & Ru-106+D & $2.17 \mathrm{E}-08$ \\
\hline 6 & $\mathrm{Na}-22$ & $8.44 E-09$ & 48 & $\mathrm{Ag}-110 \mathrm{M}+\mathrm{D}$ & 3. $19 \mathrm{E}-08$ \\
\hline 7 & $\mathrm{Na}-24$ & $1.28 \mathrm{E}-05$ & 49 & $C d-115 M$ & $1.80 \mathrm{E}-07$ \\
\hline 8 & $\mathrm{Ar}-41$ & $1.05 E-04$ & 50 & $C d-115$ & $3.60 \mathrm{E}-06$ \\
\hline 9 & Sc- -46 & $9.58 \mathrm{E}-08$ & 51 & $5 n-125+D$ & $8.31 \mathrm{E}-07$ \\
\hline 10 & $C r-5 l$ & $2.89 \mathrm{E}-07$ & 52 & Sb-124 & $1.33 \mathrm{E}-07$ \\
\hline 11 & $M n-54$ & $2.57 \mathrm{E}-08$ & 53 & Sb-125+D & $8.06 \mathrm{E}-09$ \\
\hline 12 & $M n-56$ & $7.47 \mathrm{E}-05$ & 54 & $\mathrm{Te}-132+\mathrm{D}$ & $2.47 \mathrm{E}-06$ \\
\hline 13 & $\mathrm{Fe}-55$ & $8.14 \mathrm{E}-09$ & 55 & $\mathrm{Te}-133 \mathrm{M}+\mathrm{D}$ & $2.09 \mathrm{E}-04$ \\
\hline 14 & $\mathrm{Fe}-59$ & $1.80 \mathrm{E}-07$ & 56 & $1-131+D$ & $9.97 \mathrm{E}-07$ \\
\hline 15 & Co- 57 & $2.97 \mathrm{E}-08$ & 57 & $1-132$ & $8.42 \mathrm{E}-05$ \\
\hline 16 & Co- 58 & $1.12 \mathrm{E}-07$ & 58 & $\mathrm{I}-133+\mathrm{D}$ & $9.25 \mathrm{E}-06$ \\
\hline 17 & $\mathrm{C}_{0}-60$ & $4.17 \mathrm{E}-09$ & 59 & I - 134 & $2.20 \mathrm{E}-04$ \\
\hline 18 & $\mathrm{Ni}-57$ & $5.35 \mathrm{E}-06$ & 60 & $1-135+D$ & $2.92 \mathrm{E}-05$ \\
\hline 19 & $\mathrm{Ni}-63$ & $2.20 \mathrm{E}-10$ & 61 & $\mathrm{Xe}-131 \mathrm{M}$ & $6.69 \mathrm{E}-07$ \\
\hline 20 & $\mathrm{Ni}-65$ & $7.64 \mathrm{E}-05$ & 62 & $\mathrm{Xe}-133 \mathrm{M}$ & $3.61 \mathrm{E}-06$ \\
\hline 21 & $\mathrm{Cu}-64$ & 1. $52 \mathrm{E}-05$ & 63 & $X e-133$ & $1.52 \mathrm{E}-06$ \\
\hline 22 & $2 n-65$ & $3.31 \mathrm{E}-08$ & 64 & $\mathrm{Xe}-135 \mathrm{M}$ & $7.56 \mathrm{E}-04$ \\
\hline 23 & $2 n-69 M+D$ & $1.39 \mathrm{E}-05$ & 65 & $x e-135$ & $2.10 \mathrm{E}-05$ \\
\hline 24 & As -76 & $7.32 \mathrm{E}-06$ & 66 & $\mathrm{Xe}-137$ & $3.01 \mathrm{E}-03$ \\
\hline 25 & $\mathrm{Br}-82$ & $5.44 \mathrm{E}-06$ & 67 & $X_{e}-138+D$ & 8. $14 \mathrm{E}-04$ \\
\hline 26 & $\mathrm{Kr}-83 \mathrm{M}$ & $1.04 \mathrm{E}-04$ & 68 & Cs -134 & $1.07 \mathrm{E}-08$ \\
\hline 27 & $\mathrm{Kr}-85 \mathrm{M}$ & $4.31 E-05$ & 69 & Cs-136 & $6.17 \mathrm{E}-07$ \\
\hline 28 & $\mathrm{Kr}-85$ & $2.05 \mathrm{E}-09$ & 70 & $C s-137+D$ & $7.31 \mathrm{E}-10$ \\
\hline 29 & $\mathrm{Kr}+87$ & $1.52 \mathrm{E}-04$ & 71 & Cs -138 & $3.58 \mathrm{E}-04$ \\
\hline 30 & $\mathrm{Kr}-88+\mathrm{D}$ & $6.89 \mathrm{E}-05$ & 72 & $C s-139+D$ & $1.24 \mathrm{E}-03$ \\
\hline 31 & $\mathrm{Kr}-89$ & $3.64 \mathrm{E}-03$ & 73 & $8 a-139$ & $1.39 \mathrm{E}-04$ \\
\hline 32 & $\mathrm{Rb}-88$ & $6.53 \mathrm{E}-04$ & 74 & $\mathrm{Ba}-140+\mathrm{D}$ & $6.28 \mathrm{E}-07$ \\
\hline 33 & $\mathrm{Rb}-89+\mathrm{D}$ & $7.61 \mathrm{E}-04$ & 75 & La- 140 & 4.78E-06 \\
\hline 34 & $5 r-89+D$ & $1.59 \mathrm{E}-07$ & 76 & La-14l & $4.97 \mathrm{E}-05$ \\
\hline 35 & $5 r-90+D$ & $7.58 \mathrm{E}-10$ & 77 & $\mathrm{Ce}-141$ & $2.47 \mathrm{E}-07$ \\
\hline 36 & $5 r-91+D$ & $2.03 \mathrm{E}-05$ & 78 & $\mathrm{Ce}-144+\mathrm{D}$ & $2.83 \mathrm{E}-08$ \\
\hline 37 & $5 r-92+D$ & $7.11 \mathrm{E}-05$ & 79 & $E u-152$ & $1.69 \mathrm{E}-09$ \\
\hline 38 & $Y-90$ & $3.01 \mathrm{E}-06$ & 80 & Eu-154 & $2.55 \mathrm{E}-09$ \\
\hline 39 & $Y-91 M+D$ & $2.32 \mathrm{E}-04$ & 81 & $W-187$ & $8.06 \mathrm{E}-06$ \\
\hline 40 & $2 r-95+D$ & $1.22 \mathrm{E}-07$ & 82 & Th $-232+D$ & 1. $57 \mathrm{E}-18$ \\
\hline 41 & $2 r-97+D$ & 1.14E-05 & 83 & Np-239 & $3.42 \mathrm{E}-06$ \\
\hline 42 & $\mathrm{Nb}-95$ & $2.29 \mathrm{E}-07$ & & & \\
\hline
\end{tabular}




\section{METEOROLOGY}

When more than one set of meteorological (joint frequency) data was available for a site, the one which appeared to be the most reliable was used to generate atmospheric transport factors. Factors were calculated for 16 compass points and for ten radii from ? to $80 \mathrm{~km}$ (see Table A-4) using the NRC computer program XOQDOQ (Sagendorf and Goll 1977).

TABLE A-4. Radius Intervals and Midpoints for Airborne Dose Calculations (km)

$\begin{array}{cc}\text { Interval } & \text { Midpoint } \\ 2-3 & 2.5 \\ 3-4 & 3.5 \\ 4-6 & 5 \\ 6-9 & 7.5 \\ 9-14 & 11.5 \\ 14-20 & 17 \\ 20-30 & 25 \\ 30-40 & 35 \\ 40-60 & 50 \\ 60-80 & 70\end{array}$

The $X 0 Q D O Q$ program generates four sets of atmospheric transport factors:

- average annual atmospheric dilution factors, which are not corrected for cloud depletion or radioactive decay

- dilution factors that are only corrected for decay assuming a 2.26-day half-life

- dilution factors that are corrected for depletion and for decay assuming an 8-day half-life

- relative deposition per unit area.

These factors were used to estimate the dose from airborne releases using methods similar to the NRC GASPAR program (Eckerman et al. 1980). The transport factors used this year were the same as those used for the previous estimates. The assumptions used in the calculation of these transport factors were as follows:

- 50-m source height with no correction for plume rise or building wake effects 
- semi-infinite cloud model with sector-average, Gaussian-plume dispersion

- no correction for terrain height variation.

Since information about height and locations at each site for the releases given in Tichler, et al. (1988) was unavailable, a single generic height of $50 \mathrm{~m}$ was used at each site for the release point. Because the heights and locations of releases are uncertain, estimates of dose to persons living within $2 \mathrm{~km}$ of the site could be in serious error; only persons living between 2 to $80 \mathrm{~km}$ from the site were included in the dose estimates.

\section{POPULATION}

The population distribution within 2 to $80 \mathrm{~km}$ around each site was determined from information supplied by the NRC from an updated reduction of 1980 census data (Sinisgalli 1982). Also the NRC supplied updated estimates of the number of people residing in major metropolitan centers within the $80-\mathrm{km}$ region around each site (Brauner 1982) and D. P. Cleary. (a) Population variations between 1980 and 1975 (population factors) were derived from census data (U. S. Bureau of the Census 1986).

\section{FOOD PRODUCTION VERSUS FOOD CONSUMPTION}

The total food production for the region within $80 \mathrm{~km}$ around each site was the product of the NRC state-wide productivity figure for each state and a site productivity factor. At some sites this total production may be more or less than the total consumption; i.e., population times average individual consumption (see Table A-1 for generic consumption rates). When production was more than consumption for a site, it was assumed that all persons in the $2-$ to-80-km region ate contaminated food; when production was less than consumption, it was assumed that dilution would occur because uncontaminated food would be shipped into the area from outside. Thus, the calculated doses for a particular food type were reduced in proportion to the ratio of production divided by consumption (production/consumption less than 1).

The dose to persons outside the $80-\mathrm{km} 1 \mathrm{imit}$ from food shipped out of the region, in the case of production being greater than consumption, is not included in this report because we are concerned only with the dose within the $80-\mathrm{km}$ radius. These production/consumption factors are given for reference as footnotes to the tables showing airborne dose commitment in the Site Summaries section.

(a) Letter from D. P. Cleary, U.S. Nuclear Regulatory Commission, to D. A. Baker, Pacific Northwest Laboratory, June 1987. 


\section{DRINKING WATER}

The population between 2 and $80 \mathrm{~km}$ distance from each plant site exposed to drinking water contaminated with released radionuclides was generally obtained from the environmental statement (ES) for the plant. For all sites located on salt water, it was assumed that no dose was received from drinking water. The generic consumption rates used for drinking water are given in Table A-1.

The radionuclide concentration in the drinking water consumed by a population downstream from a site was usually estimated assuming $100 \%$ mixing of the plant effluent with the river. For lakes, an overall dilution factor was estimated from dilution factors given in the ES for each population center along the shore (within $80 \mathrm{~km}$ ) that consumes the contaminated lake water. These individual factors were weighted by population and averaged to obtain an effective dilution factor for the total population exposed to contaminated drinking water.

\section{AQUATIC FOOD}

Wherever possible, the fish-catch data from the plant ES were used to estimate aquatic food consumption rates for the population living within the region. When these data were not found in the ES or were considered unrealistic, the generic values of Table A-1 were used.

The average radionuclide concentration of the waters in which this food was harvested was estimated assuming an additional dilution over the effluent flow from the reactor. For rivers, it was assumed that the fish were caught in waters in which the plant effluent was completely diluted. For lakes, an additional factor as given in the ES was used; when none was given in the ES, a generic value of 0.01 was used. For ocean and bay sites, generic values of 0.001 and 0.002 were used for $f i s h$ and invertebrates, respectively, if the ES yielded no values for these parameters. Invertebrates were not assumed to be caught in sufficient quantity at freshwater sites (river and lake) to affect the population dose and therefore were not included in the dose calculation. Any exceptions to these general guidelines are explained in the footnotes to the individual site summaries.

\section{TECHNICAL NOTES}

The calculations leading to the dose estimates contained in this report were generated from recent versions of computer programs originally documented in Baker et a1. (1977). The revised programs were written in BASICA and run on an IBM PC microcomputer operating under the PC-DOS operating system. The text and charts were all generated by an HP LaserJet II printer. 


\section{APPENDIX REFERENCES}

Baker, D. A., J. K. Soldat and E. C. Watson. 1977. Population-Dose Commitments Due to Radioactive Releases from Nuclear Power Plant Sites in 1975. PNL-2439, Pacific Northwest Laboratory, Richland, Washington.

Brauner, A. 1982. Population Estimates, Nuclear Power Plant Nearby Population Concentrations. U.S. Nuclear Regulatory Commission, Washington, D.C.

Eckerman, K. F., F. J. Conge], A. K. Roecklein and W. J. Pasciak. 1980. Users Guide to GASPAR Code. NUREG-0597, U.S. Nuclear Regulatory Comission, Washington, D.C.

Hoenes, G. R., and J. K. Soldat. 1977. Age-Specific Radiation Dose Commitment Factors for a One-Year Chronic Intake. NUREG-0172, U.S. Nuclear Regulatory Commission, Washington, D.C.

Population Estimates and Projections, Current Population Reports. 1975. Series P-25, No. 541. U.S. Department of Commerce, Social and Economic Statistics Administration, Bureau of the Census.

Sagendorf, J. F., and J. T. Goll. 1977. X00D00--Program for the Meteorological Evaluation of Routine Effluent Releases at Nuclear Power Stations. NUREG-0324, Draft, U.S. Nuclear Regulatory Commission, Washington, D.C.

Sinisgalli, A. 1982. $\quad 1980$ Residential Population Estimates, $\quad 0-80$ Kilometers for Nuclear Power Plants. U. S. Nuclear Regulatory Commission, Washington, D.C.

Tichler, J., K. Norden, and J. Congemi. 1988. Radioactive Materials Released from Nuclear Power Plants, Annual Report 1985. NUREG-CR-2907, BNL-NUREG-51581, Vol. 6, U.S. Nuclear Regulatory Commission, Washington, D.C.*

U.S. Bureau of the Census. 1986. Statistical Abstract of the United States: 1987, 107th edition. U.S. Department of Commerce, Washington, D.C.

U.S. Nuclear Regulatory Commission. 1977. Calculation of Annual Doses to Man from Routine Releases of Reactor Effluents for the Purpose of Evaluating Compliance with 10 CFR Part 50, Appendix 1, Revision 1. Regulatory Guide 1.109, Washington, D.C. 



\begin{tabular}{|c|c|}
\hline 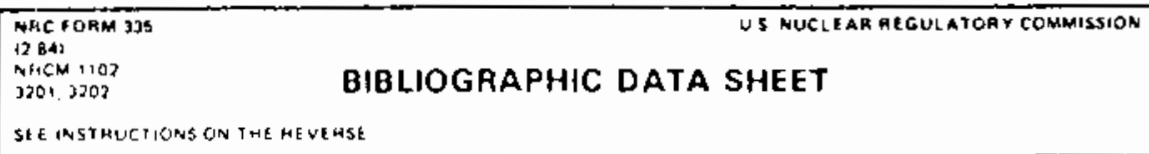 & 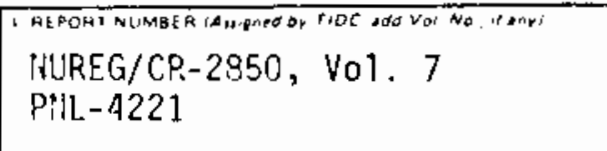 \\
\hline \multirow{3}{*}{$\begin{array}{l}\text { Population Dose Commitments Due to Radioactive Releases } \\
\text { from Nuclear Power Plant Sites in } 1985\end{array}$} & \multirow[t]{2}{*}{ 3LEAYE BLANK } \\
\hline & \\
\hline & \begin{tabular}{l|l} 
MONIM & 1988 \\
Alugust & \\
\end{tabular} \\
\hline 7. A. Baker & \begin{tabular}{c|c} 
SDATE REPORT ISSUEO \\
MUNIH & YEAR \\
AuguSt & 1988
\end{tabular} \\
\hline 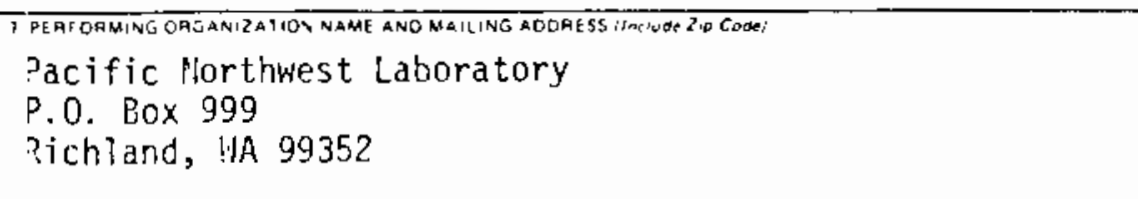 & $\begin{array}{l}\text { 9FIN DA G̈ANT NUMAEF } \\
\text { B2243 }\end{array}$ \\
\hline 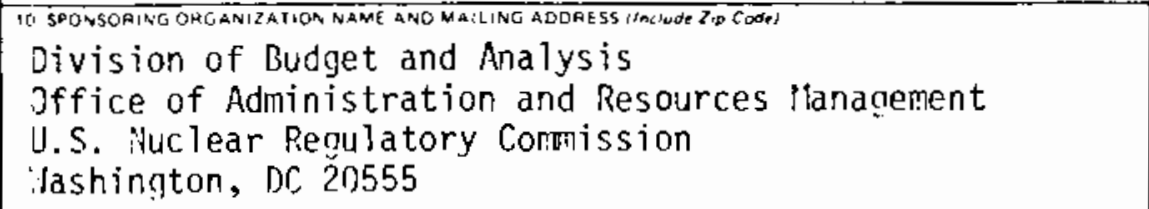 & 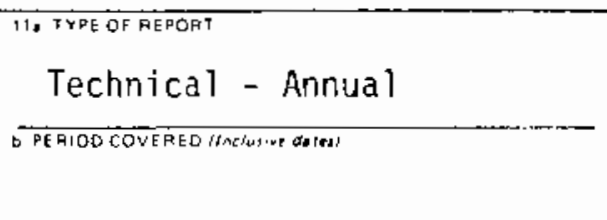 \\
\hline \multicolumn{2}{|l|}{ 1. SUPPLEMEATARY NOTES } \\
\hline \multicolumn{2}{|c|}{$\begin{array}{l}\text { Population radiation dose commitments have been estimated from reported radionuclide } \\
\text { releases from commercial power reactors operating during } 1985 \text {. Fifty-year dose } \\
\text { commitments from a one-year exposure were calculated from both liquid and atmospheric } \\
\text { releases for four population groups (infant, child, teen-ager and adult) residing between } \\
2 \text { and } 80 \mathrm{~km} \text { from each of } 61 \text { sites. This report tabulates the results of these } \\
\text { calculations, showing the dose commitments for both liquid and airborne pathways for } \\
\text { each age group and organ. Also included for each of the sites is a histogram showing } \\
\text { the fraction of the total population within } 2 \text { to } 80 \mathrm{~km} \text { around each site receiving various } \\
\text { average dose commitments from the airborne pathways. } \\
\text { The total dose commitments (from both liquid and airborne pathways) for each site ranged } \\
\text { from a high of } 73 \text { person-rem to a low of } 0.011 \text { person-rem for the sites with plants } \\
\text { operating throughout the year with an arithmetic mean of } 3 \text { person-rem. The total } \\
\text { population dose for all sites was estimated at } 200 \text { person-rem for the } 110 \text { million people } \\
\text { considered at risk. } \\
\text { The site average individual dose commitment from all pathways ranged from a low of } \\
5 \text { x io-6 mrem to a high of } 0.02 \text { mrem. No attempt was made in this study to determine } \\
\text { the maximum dose commitment received by any one individual from the radionuclides } \\
\text { released at any of the sites. }\end{array}$} \\
\hline \multirow[t]{3}{*}{ 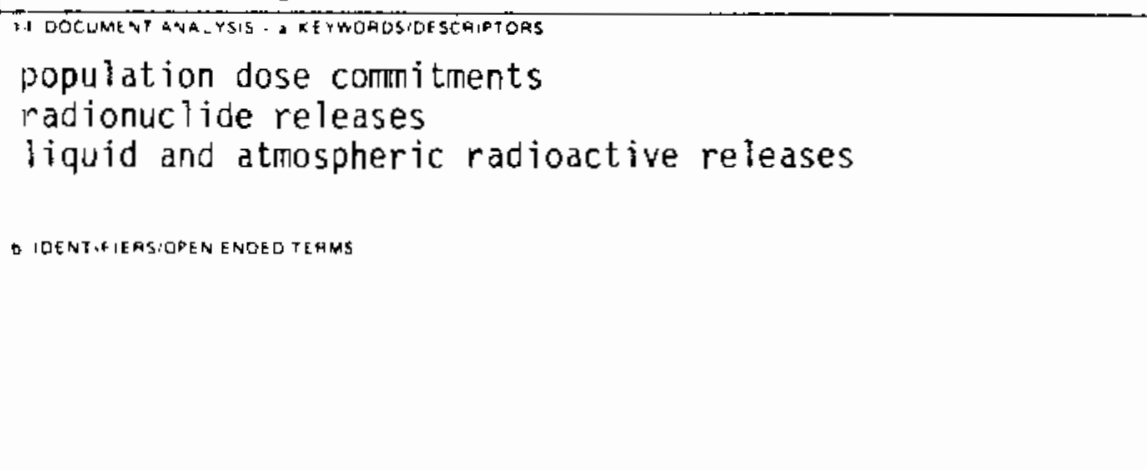 } & {$\left[\begin{array}{c}\text { STAALABCITY } \\
\text { STATEMENT } \\
\text { Unlimited }\end{array}\right.$} \\
\hline & 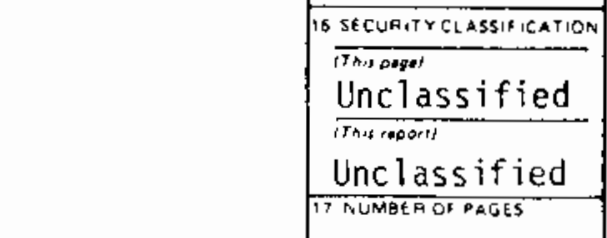 \\
\hline & 18 PRICE \\
\hline
\end{tabular}


